\title{
Concise Synthesis of 9,11-Secosteroids Pinnigorgiols B and E
}

\author{
Xinghui Li, ${ }^{1, \dagger}$ Zeliang Zhang,,${ }^{1, \dagger}$ Huafang Fan, ${ }^{2}$ Yinlong Miao, ${ }^{2}$ Hailong Tian, ${ }^{1}$ Yucheng $\mathrm{Gu}^{3}$ and \\ Jinghan Gui*,1
}

\begin{abstract}
${ }^{1}$ CAS Key Laboratory of Synthetic Chemistry of Natural Substances, Center for Excellence in Molecular Synthesis, Shanghai Institute of Organic Chemistry, University of Chinese Academy of Sciences, Chinese Academy of Sciences, 345 Lingling Road, Shanghai, 200032, China. ${ }^{2}$ Shaanxi Key Laboratory of Natural Products \& Chemical Biology, College of Chemistry \& Pharmacy, Northwest A\&F University, 22 Xinong Road, Yangling 712100, Shaanxi, China. ${ }^{3}$ Syngenta Jealott's Hill International Research Centre, Bracknell, Berkshire RG42 6EY, United Kingdom.
\end{abstract}

†These authors contributed equally to this work. *Correspondence to: guijh@ sioc.ac.cn.

\section{Supporting Information}

\section{Table of Contents}

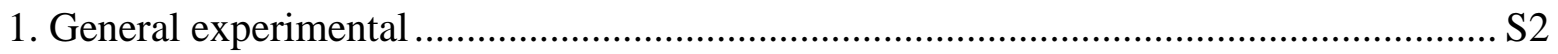

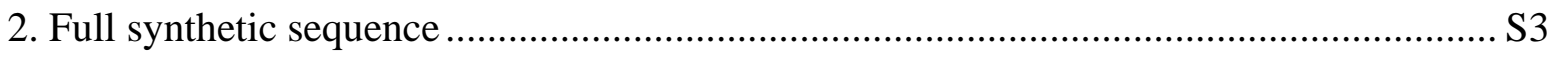

3. Additional experiments for structural elucidation and mechanistic studies................... S6

4. Experimental procedures and characterization data for new compounds.......................S9

5. NMR data comparison of synthetic and natural pinnigorgiols $\mathrm{B}$ and E ......................S34

6. NMR spectra comparison of synthetic and natural pinnigorgiols B and E...................S38

7. ECD spectra of natural and synthetic pinnigorgiols B and E ..................................S46

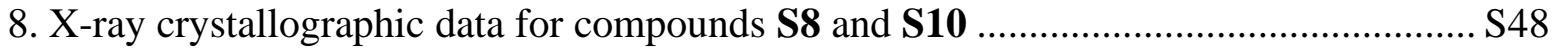

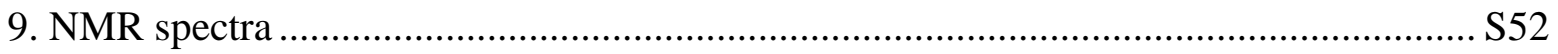




\section{General experimental}

All reactions utilizing air- or moisture-sensitive reagents were carried out in flame-dried glassware under an argon atmosphere, unless otherwise stated. Dry tetrahydrofuran (THF), dichloromethane (DCM), N,N-Dimethylformamide (DMF) were obtained by passing the HPLC grade or pre-dried solvents through activated alumina columns. Pyridine, chloroform $\left(\mathrm{CHCl}_{3}\right)$, cyclohexane, ethanol $(\mathrm{EtOH})$ and triethylamine $\left(\mathrm{Et}_{3} \mathrm{~N}\right)$ were distilled from $\mathrm{CaH}_{2}$. Reagents were purchased at the highest commercial quality and used without further purification, unless otherwise stated. Reactions were magnetically stirred and monitored by thin layer chromatography (TLC) with 0.15-0.2 mm pre-coated silica gel $(10-40 \mu \mathrm{m})$ plates, using UV light as the visualizing agent or ethanolic phosphomolybdic acid and heating as developing agents. Flash chromatography was performed with silica gel (200-300 mesh) under pressure. Yields refer to chromatographically and spectroscopically $\left({ }^{1} \mathrm{H}\right.$ NMR) homogeneous material, unless otherwise stated. NMR spectra were recorded on Bruker-400, Agilent-400 and Bruker-500 spectrometers. ${ }^{1} \mathrm{H}$ NMR spectra were calibrated using residual undeuterated solvent as an internal reference $\left(\mathrm{CDCl}_{3}: 7.26 \mathrm{ppm}\right)$ and ${ }^{13} \mathrm{C} \mathrm{NMR}$ spectra were calibrated against the deuterated solvent peak $\left(\mathrm{CDCl}_{3}: 77.2 \mathrm{ppm}\right)$. The following abbreviations were used to explain multiplicities: $\mathrm{s}=$ singlet, $\mathrm{d}=$ doublet, $\mathrm{t}=$ triplet, $\mathrm{q}=$ quartet, $\mathrm{m}=$ multiplet, br = broad. IR spectra were collected on Avatar 330 FT-IR spectrometer. Melting points were determined on SGW X-4 microscopic melting point apparatus and were uncorrected. Optical rotations were determined on JASCO P-1030 Polarimeter in the solvent indicated. High-resolution mass spectra were recorded on IonSpec 4.7 Tesla FTMS or Bruker Daltonics, Inc. APEXIII 7.0 TESLA FTMS. 


\section{Full synthetic sequence}

\section{Scheme S1. Acyl Radical Cyclization Approach to Pinnigorgiols B and E}

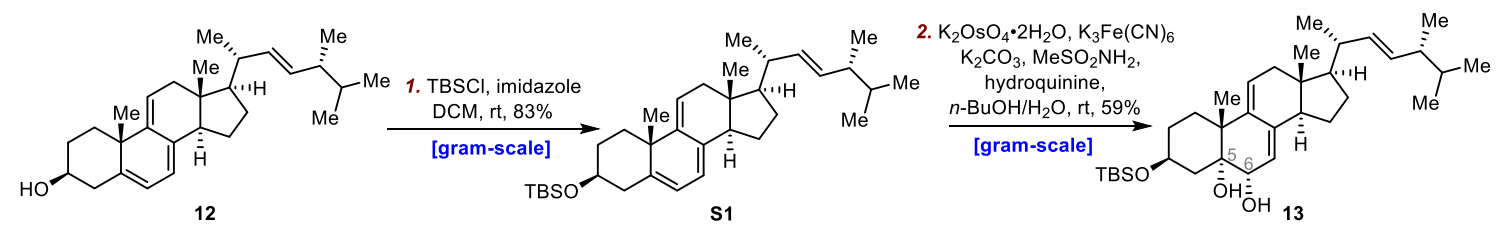

3. $10 \% \mathrm{Rh} / \mathrm{C}, \mathrm{H}_{2}$
EtOAc, $30^{\circ} \mathrm{C}, 96 \%$

[gram-scale]
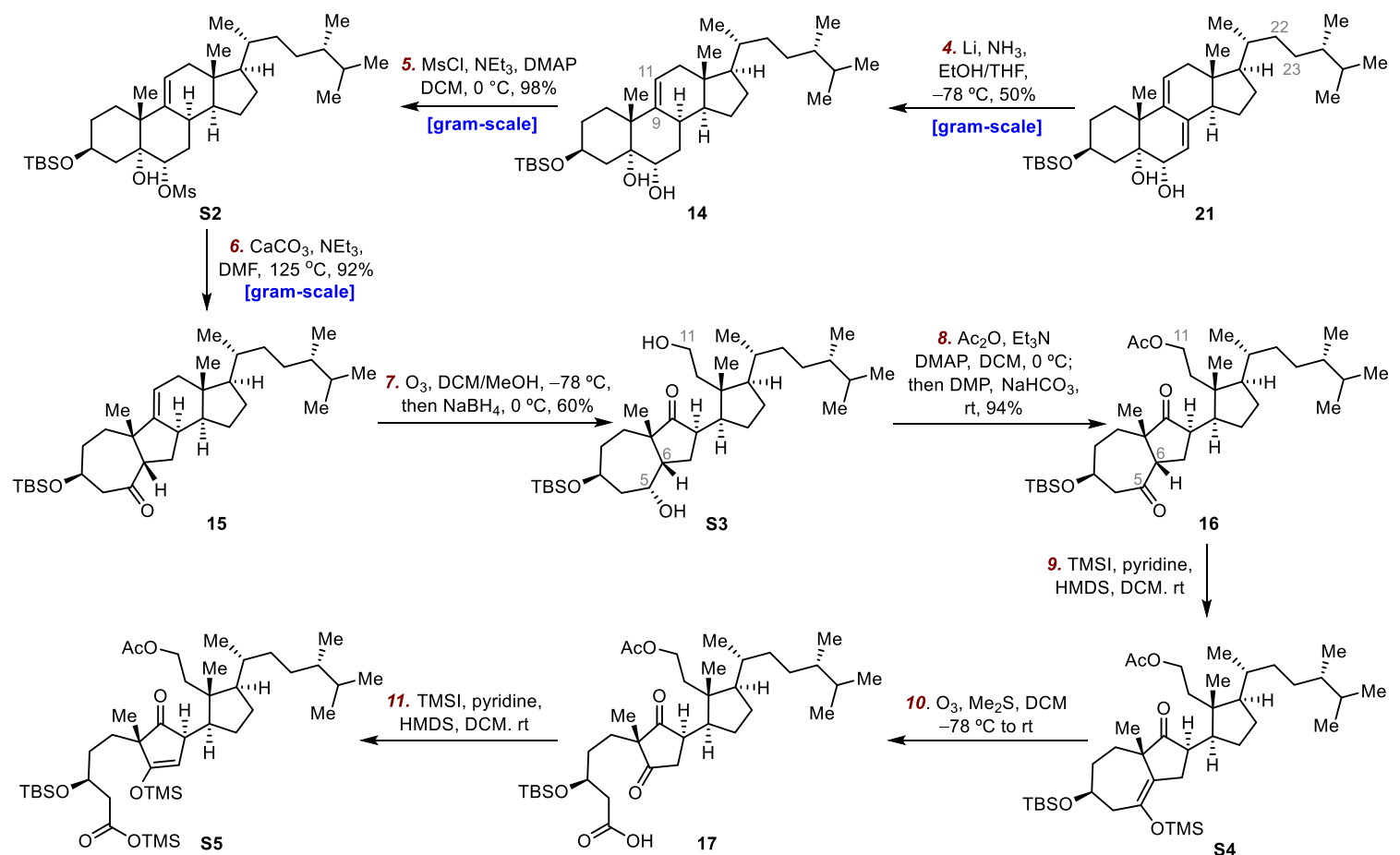

$\downarrow \begin{gathered}\text { 12. NBS, THF, } \\ 0{ }^{\circ} \mathrm{C}, 58 \% \text { (4 steps) }\end{gathered}$

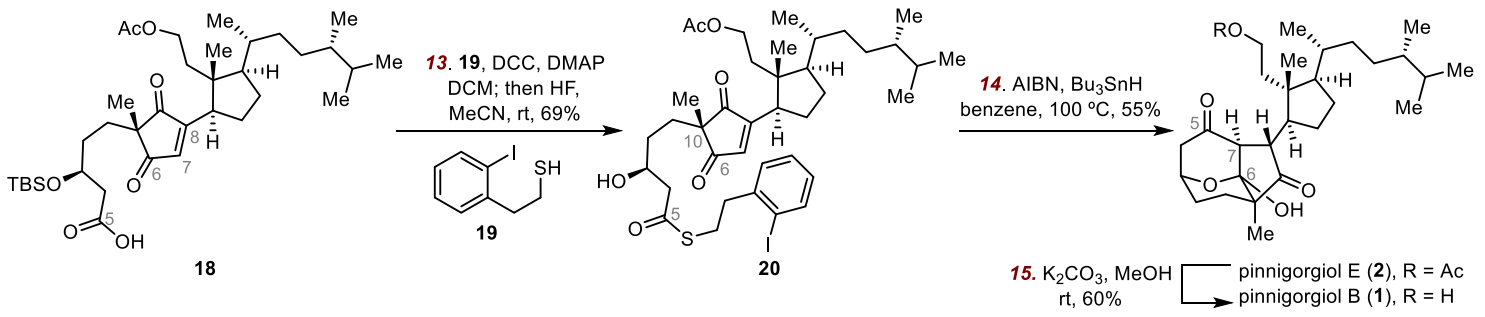

Reagents and conditions: (1) TBSCl (1.5 equiv), imidazole (3.0 equiv), DCM, rt, $2 \mathrm{~h}, 83 \%$; (2) $\mathrm{K}_{2} \mathrm{OsO}_{4} \cdot 2 \mathrm{H}_{2} \mathrm{O}$ (0.3 equiv), $\mathrm{K}_{3} \mathrm{Fe}(\mathrm{CN})_{6}$ (10.0 equiv), $\mathrm{K}_{2} \mathrm{CO}_{3}$ (10.0 equiv), $\mathrm{MeSO}_{2} \mathrm{NH}_{2}$ (1.0 equiv), hydroquinine (2.0 equiv), $n-\mathrm{BuOH} / \mathrm{H}_{2} \mathrm{O}(1 / 1 \mathrm{v} / \mathrm{v}), \mathrm{rt}, 24 \mathrm{~h}, 59 \%$; (3) $10 \% \mathrm{Rh} / \mathrm{C}$ (30 wt $\left.\%\right), \mathrm{H}_{2}$ (1 atm), EtOAc, $30^{\circ} \mathrm{C}, 13 \mathrm{~h}, 96 \%$; (4) Li wire (10 equiv $\left.\times 8\right)$, THF/EtOH (1/1 v/v), $-78{ }^{\circ} \mathrm{C}, 4 \mathrm{~h}, 50 \%$; (5) $\mathrm{MsCl}$ (2.0 equiv), $\mathrm{Et}_{3} \mathrm{~N}$ (4.0 equiv), DMAP (0.1 equiv), DCM, $0{ }^{\circ} \mathrm{C}, 10 \mathrm{~min}, 98 \%$; (6) $\mathrm{CaCO}_{3}$ (4.5 equiv), 
$\mathrm{Et}_{3} \mathrm{~N}$ (6.0 equiv), DMF, $125{ }^{\circ} \mathrm{C}, 12 \mathrm{~h}, 92 \%$; (7) $\mathrm{O}_{3}, \mathrm{DCM} / \mathrm{MeOH}(5 / 1 \mathrm{v} / \mathrm{v}),-78{ }^{\circ} \mathrm{C}$; then $\mathrm{NaBH}_{4}(2.0$ equiv), $-78{ }^{\circ} \mathrm{C}$ to $0{ }^{\circ} \mathrm{C}, 1 \mathrm{~h}, 60 \%$; (8) $\mathrm{Ac}_{2} \mathrm{O}$ (1.2 equiv), $\mathrm{Et}_{3} \mathrm{~N}$ (3.0 equiv), DMAP (0.1 equiv), DCM, 0 ${ }^{\circ} \mathrm{C}, 30 \mathrm{~min}$; then DMP (5.0 equiv), $\mathrm{NaHCO}_{3}$ (10.0 equiv), $0{ }^{\circ} \mathrm{C}$ to $\mathrm{rt}, 1 \mathrm{~h}, 94 \%$; (9) TMSI (4.5 equiv), HMDS (6.0 equiv), pyridine ( 3.0 equiv), DCM, $0{ }^{\circ} \mathrm{C}$ to rt, $30 \mathrm{~min}$; then TMSI (4.5 equiv), HMDS (6.0 equiv), pyridine ( 3.0 equiv), rt, $30 \mathrm{~min}$; (10) $\mathrm{O}_{3}, \mathrm{Me}_{2} \mathrm{~S}$ (3.0 equiv), DCM, $-78{ }^{\circ} \mathrm{C}$ to rt, $5 \mathrm{~h}$; (11) TMSI (4.5 equiv), HMDS (6.0 equiv), pyridine (3.0 equiv), DCM, $0{ }^{\circ} \mathrm{C}$ to rt, $30 \mathrm{~min}$; (12) NBS (1.1 equiv), THF, $0{ }^{\circ} \mathrm{C}, 10 \mathrm{~min}, 58 \%$ (4 steps); (13) 19 (1.4 equiv), DCC (1.4 equiv), DMAP (0.2 equiv), DCM, rt; then $40 \%$ aq. $\mathrm{HF}$ (280.0 equiv), MeCN, rt, 69\% ; (14) $\mathrm{AIBN}$ (1.0 equiv), $\mathrm{Bu}_{3} \mathrm{SnH}$ (2.2 equiv), benzene, $100{ }^{\circ} \mathrm{C}, 1 \mathrm{~h}, 55 \%$; (15) $\mathrm{K}_{2} \mathrm{CO}_{3}$ (1.5 equiv), $\mathrm{MeOH}, \mathrm{rt}, 60 \%$. Abbreviation: TBS, tert-butyldimethylsilyl; DCM, dichloromethane; THF, tetrahydrofuran; Ms, mesyl; $\mathrm{Et}_{3} \mathrm{~N}$, triethylamine; DMF, $\mathrm{N}, \mathrm{N}$-dimethylformamide; DMAP, $\mathrm{N}, \mathrm{N}$-4-dimethylaminopyridine; DMP, Dess-Martin periodinane; TMS, trimethylsilyl; HMDS, hexamethyldisilazane; NBS, $N$-bromosuccinimide; DCC, $N, N^{\prime}$-dicyclohexylcarbodiimide; AIBN, 2,2'-azoisobutyronitrile.

\section{Scheme S2. Regioselective-Hydrogenation-Enabled Divergent Access to Regioisomeric Olefins}
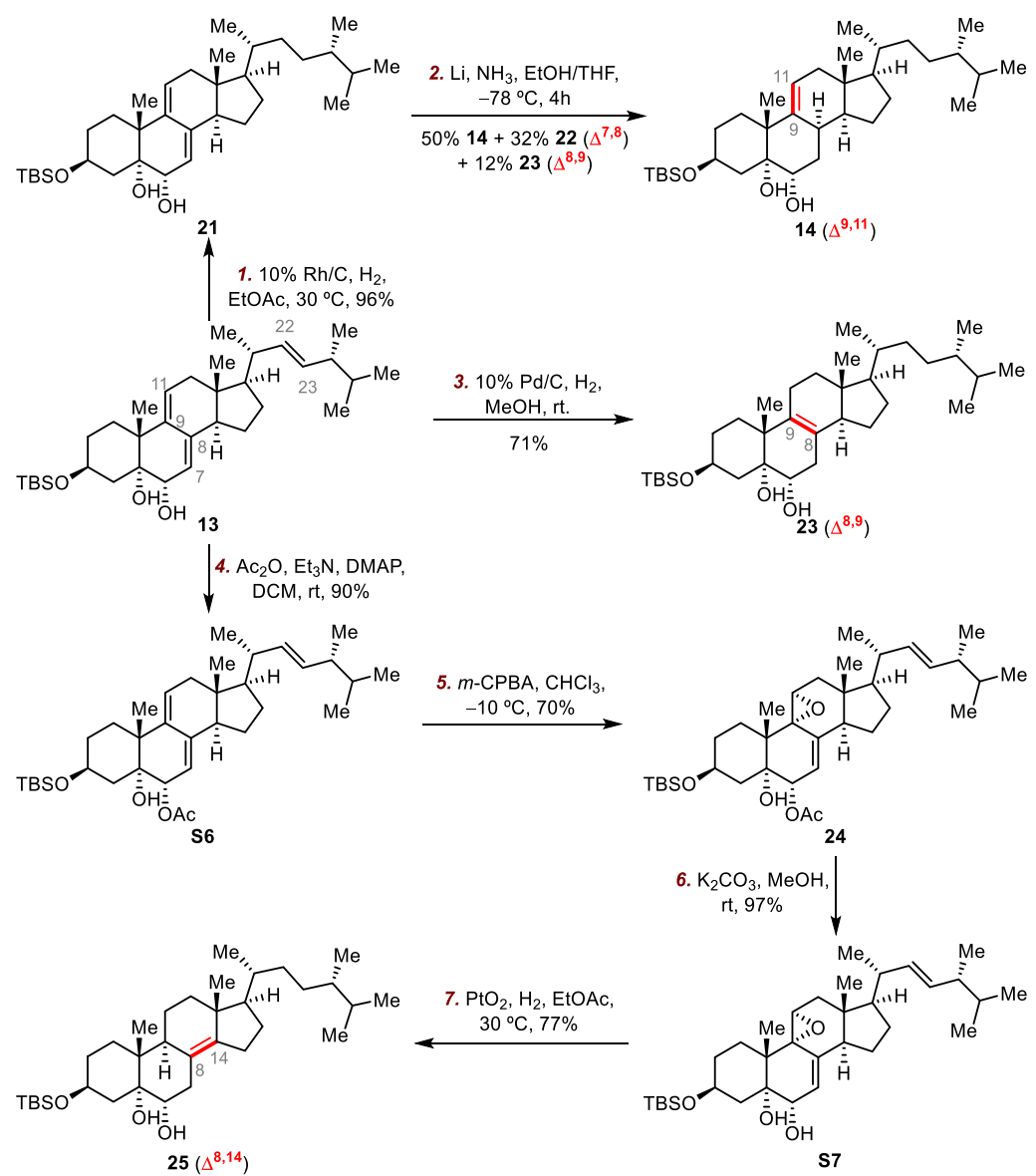

Reagents and conditions: (1) $10 \% \mathrm{Rh} / \mathrm{C}(30 \mathrm{wt} \%), \mathrm{H}_{2}(1 \mathrm{~atm})$, EtOAc, $30{ }^{\circ} \mathrm{C}, 23 \mathrm{~h}, 96 \%$; (2) Li wire (10 equiv $\times 8)$, THF/EtOH (1/1 v/v), $-78{ }^{\circ} \mathrm{C}, 4 \mathrm{~h}, 50 \% 14+32 \% 22+12 \% \mathbf{2 3}$; (3) 10\% Pd/C (40 wt $\left.\%\right)$, $\mathrm{H}_{2}$ (1 atm), MeOH, rt, $11 \mathrm{~h}, 71 \%$; (4) $\mathrm{Ac}_{2} \mathrm{O}$ (1.5 equiv), $\mathrm{Et}_{3} \mathrm{~N}$ (3.0 equiv), DMAP (0.1 equiv), DCM, rt, 
$1 \mathrm{~h}, 90 \%$; (5) m-CPBA (1.5 equiv), $\mathrm{CHCl}_{3},-10{ }^{\circ} \mathrm{C}, 9 \mathrm{~h}, 70 \%$; (6) $\mathrm{K}_{2} \mathrm{CO}_{3}$ (2.0 equiv), $\mathrm{MeOH}, \mathrm{rt}, 1 \mathrm{~h}$, 97\%; (7) $\mathrm{PtO}_{2}(50 \mathrm{wt} \%), \mathrm{H}_{2}(1 \mathrm{~atm})$, EtOAc, $30{ }^{\circ} \mathrm{C}, 20 \mathrm{~h}, 77 \%$. Abbreviation: $m-\mathrm{CPBA}$, meta-chloroperbenzoic acid. 


\section{Additional experiments for structural elucidation and mechanistic studies}

1) Structural elucidation of olefins 23 and 25

To elucidate the structure of olefins $\mathbf{2 3}$ and $\mathbf{2 5}$, we performed the semipinacol rearrangements of $\mathbf{2 3}$ and $\mathbf{2 5}$, and the structures of products S8 and S10 were unambiguously determined by X-ray crystallographic analysis. These two $\mathrm{x}$-ray structures also confirmed the stereochemistry at C6 for the semipinacol rearrangement products 15, and S8-S10.

\section{Scheme S3. Structural Elucidation of Olefins 23 and 25}

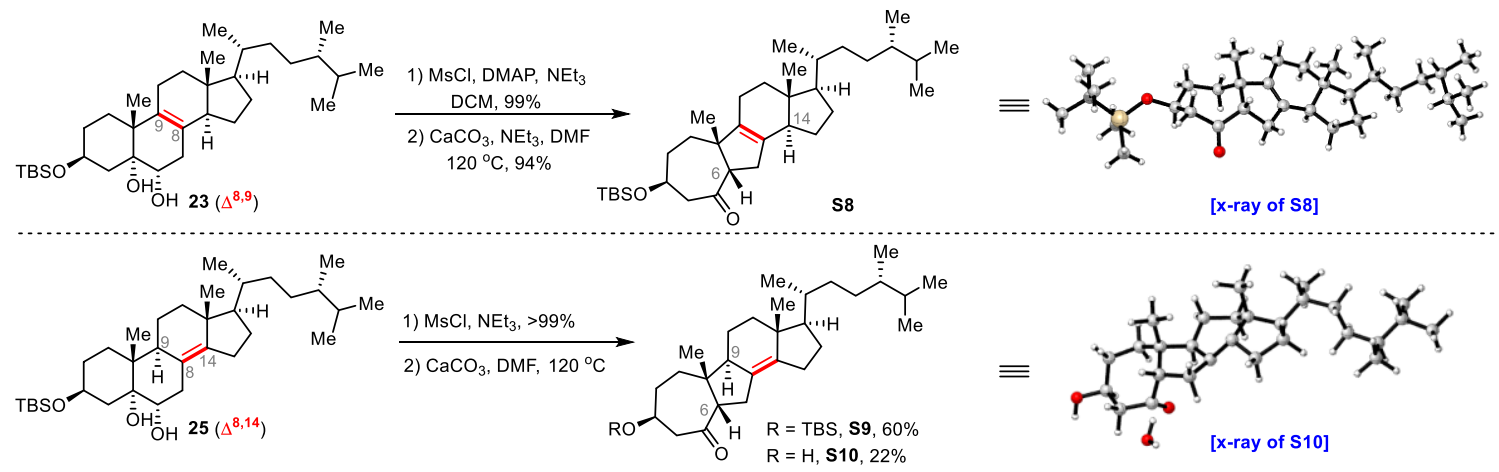

2) Mechanistic rationale for the selective formation of $\mathbf{1 4 , 2 3}$ and 25

We speculated that the reduction of 7,9-diene 21 by $\mathrm{Li} / \mathrm{NH}_{3}$ proceeded in a 1,2-manner to afford C9-C11 olefin 14 as major product and C7-C8 olefin 22 as minor product (Scheme 3, main text); the reduction of 7,9-diene $\mathbf{1 3}$ by $10 \% \mathrm{Pd} / \mathrm{C}$ and $\mathrm{H}_{2}$ proceeded in a 1,4-manner to give C8-C9 olefin 23 exclusively. However, the selective formation of C8-C14 olefin 25 is a more complicated process. In order to identify possible intermediates, we performed several control experiments (Scheme S4): 1) treatment of 22 or 23 with $\mathrm{PtO}_{2}$ and 1 atm $\mathrm{H}_{2}$ at $30{ }^{\circ} \mathrm{C}$ generated $\mathbf{2 5}$ in excellent yields; 2) when $\mathbf{S 7}$ was hydrogenated for a short period of time (1 h), $\mathbf{2 5}$ and $\mathbf{2 3}$ were isolated in 51\% and 35\% yields, respectively; and 3) no reaction occurred when 9,11-epoxide S11 was subjected to the same conditions. 
On the basis of the above results, we surmised that the hydrogenation of $\mathbf{S 7}$ by $\mathrm{PtO}_{2}$ and $\mathrm{H}_{2}$ first generated allylic alcohol S12, rather than 9,11-epoxide S11. S12 could in turn be transformed to $\mathrm{C} 8$-C14 olefin 25 via two pathways: 1) dehydration of the C11-hydroxyl group to give $\mathbf{S 1 3}$, followed by selective hydrogenation of the C9-C11 olefin (path a); and 2) hydrogenation of the C11-allylic alcohol of S12 to give C8-C9 olefin 23, followed by olefin isomerization. Our control experiments showed that C8-C9 olefin $\mathbf{2 3}$ was the intermediate of the transformation from $\mathbf{S 7}$ to $\mathbf{2 5}$, suggesting path b was more likely.

Scheme S4. Mechanistic Rationale for the Selective Formation of 25

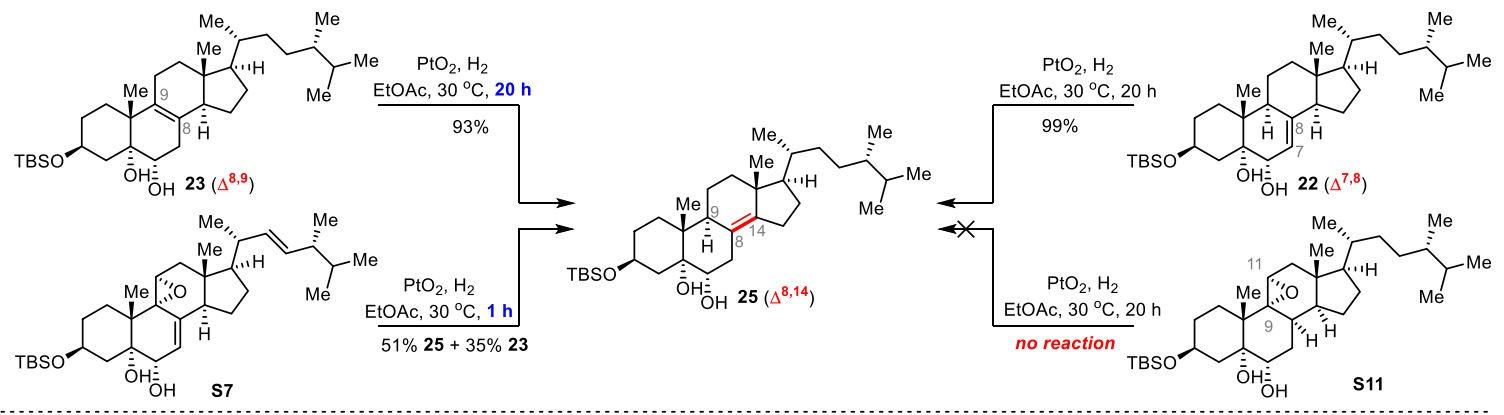

Proposed pathways for the formation of $\mathbf{2 5}$ :
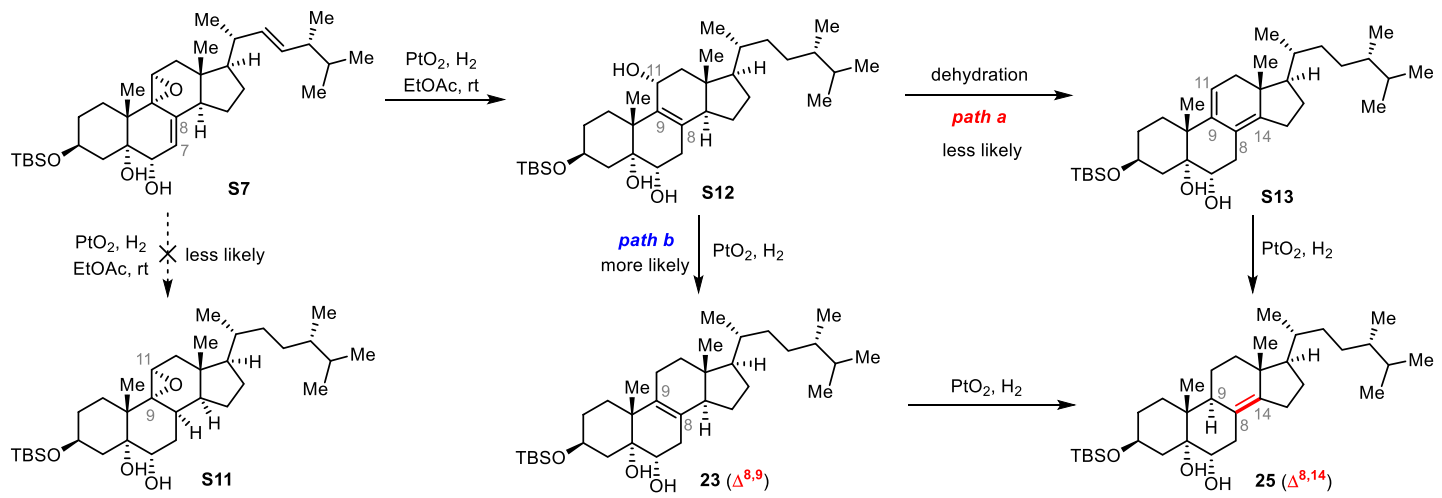

3) Mechanistic study of the key acyl radical cyclization

The key acyl radical cyclization/hemiketalization cascade to convert $\mathbf{2 0}$ to pinnigorgiol E (2) could proceed via two pathways (Scheme S5): 1) acetyl radical cyclization to form the 8-membered ring preceded the hemiketal formation (path a), and 2) the hemiketal 
formation preceded the acetyl radical cyclization (path b). To investigate the order of the two reactions, we performed the radical cyclization on the TBS-protected substrate $\mathbf{S 1 6}$ and obtained a cyclized product $\mathbf{S 1 7}$, which could be converted to pinnigorgiol E (2) in 14\% yield over 2 steps upon treatment with $40 \% \mathrm{HF}$ in MeCN. In addition, we didn't observe any signals corresponding to the hemiketal isomer of 20 from its ${ }^{13} \mathrm{C} \mathrm{NMR}$ spectrum. Therefore, it's likely that the 8 -membered ring cyclization preceded the hemiketal formation in the key acyl radical cyclization/hemiketalization cascade. [We thank one of the anonymous reviewers for suggesting this control experiment.]

\section{Scheme S5. Mechanistic study of the Key Acyl Radical Cyclization}
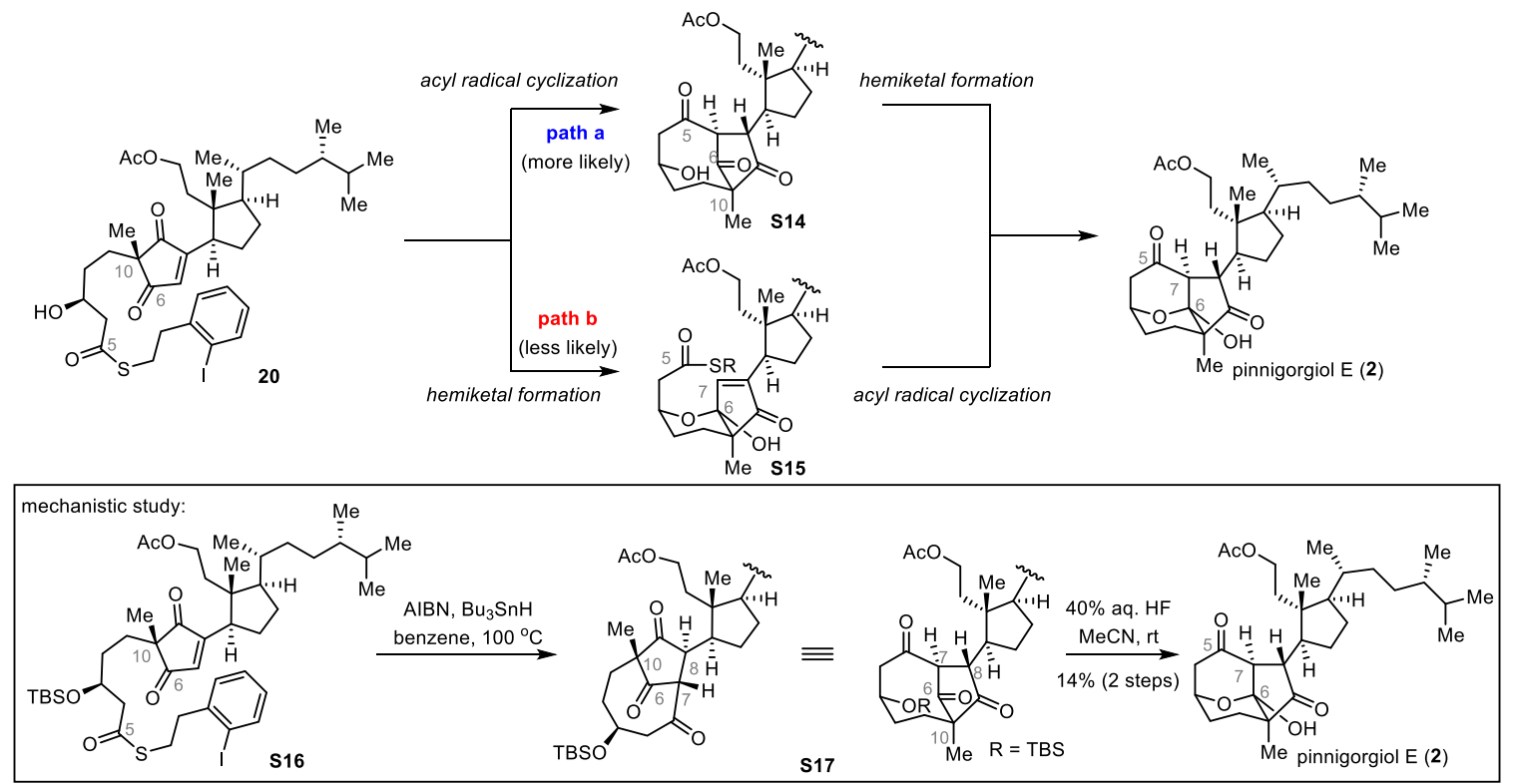


\section{Experimental procedures and characterization data for new compounds}

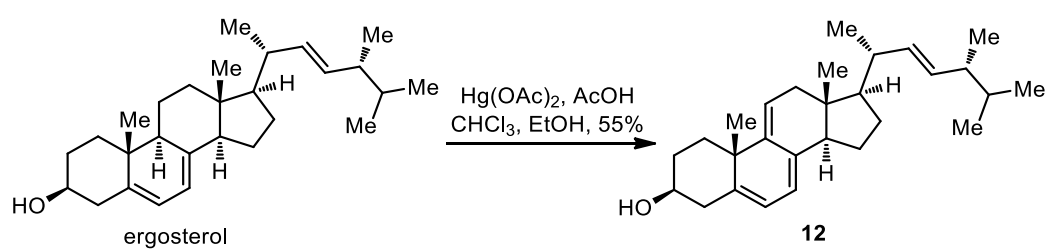

To a solution of ergosterol ( $8.0 \mathrm{~g}, 20.2 \mathrm{mmol}, 1.0$ equiv) in $336 \mathrm{~mL}$ EtOH and $240 \mathrm{~mL}$ $\mathrm{CHCl}_{3}$ was added $\mathrm{AcOH}\left(0.19 \mathrm{~mL}, 3.6 \mathrm{mmol}, 0.18\right.$ equiv) and $\mathrm{Hg}(\mathrm{OAc})_{2}(19.2 \mathrm{~g}, 60.5$ mmol, 3.0 equiv) at room temperature. The resulting mixture was stirred for $40 \mathrm{~h}$ in dark before it was filtered through celite. Removal of the solvent under reduced pressure afforded the crude product, which was purified by flash chromatography $\left(\mathrm{SiO}_{2}, 20: 1\right.$ petroleum ether:EtOAc) to provide $12(4.4 \mathrm{~g}, 55 \%)$ as a white solid.

Compound 12: TLC (petroleum ether:EtOAc, 2:1 v/v): $R_{f}=0.50 ;{ }^{1} \mathrm{H}$ NMR $(400 \mathrm{MHz}$, Chloroform- $d$ ) $\delta 5.67(\mathrm{dd}, J=5.6,1.2 \mathrm{~Hz}, 1 \mathrm{H}), 5.51(\mathrm{~d}, J=6.0 \mathrm{~Hz}, 1 \mathrm{H}), 5.40(\mathrm{~d}, J=5.6$ $\mathrm{Hz}, 1 \mathrm{H}), 5.23(\mathrm{dd}, J=15.2,7.2 \mathrm{~Hz}, 1 \mathrm{H}), 5.16(\mathrm{dd}, J=15.2,7.6 \mathrm{~Hz}, 1 \mathrm{H}), 3.60(\mathrm{tt}, J=10.7$, $4.8 \mathrm{~Hz}, 1 \mathrm{H}), 2.46(\mathrm{t}, J=12.0 \mathrm{~Hz}, 1 \mathrm{H}), 2.41-2.30(\mathrm{~m}, 2 \mathrm{H}), 2.27-2.12(\mathrm{~m}, 2 \mathrm{H}), 2.11-$ $1.97(\mathrm{~m}, 1 \mathrm{H}), 1.96-1.89(\mathrm{~m}, 1 \mathrm{H}), 1.89-1.64(\mathrm{~m}, 6 \mathrm{H}), 1.57-1.27(\mathrm{~m}, 4 \mathrm{H}), 1.24(\mathrm{~s}, 3 \mathrm{H})$, $1.02(\mathrm{~d}, J=6.8 \mathrm{~Hz}, 3 \mathrm{H}), 0.91(\mathrm{~d}, J=6.8 \mathrm{~Hz}, 3 \mathrm{H}), 0.84(\mathrm{~d}, J=6.4 \mathrm{~Hz}, 3 \mathrm{H}), 0.83(\mathrm{~d}, J=$ $6.4 \mathrm{~Hz}, 3 \mathrm{H}), 0.58$ (s, 3H).

Spectroscopic data was identical to that reported in the literature (Helv. Chim. Acta 1979, 62, 2037; J. Am. Chem. Soc. 1953, 75, 2604).

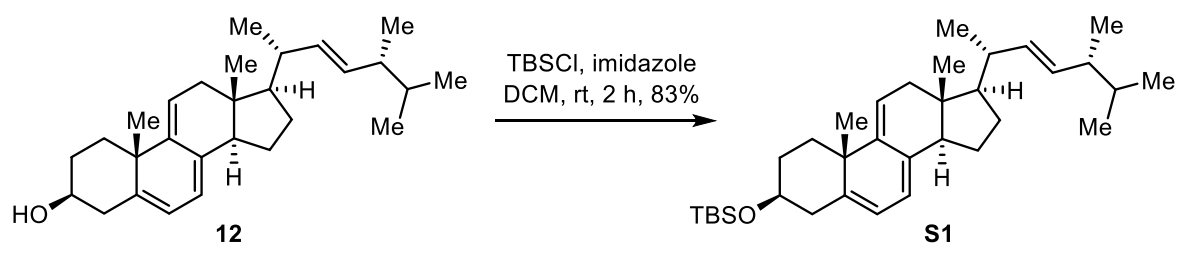

To a solution of 12 (12.9 g, $30.1 \mathrm{mmol}, 1.0$ equiv) in $100 \mathrm{~mL}$ DCM was added imidazole (6.1 g, $90.3 \mathrm{mmol}, 3.0$ equiv) and $\mathrm{TBSCl}(6.8 \mathrm{~g}, 45.1 \mathrm{mmol}, 1.5 \mathrm{gequiv})$ at room temperature. After stirred for $2 \mathrm{~h}$, the reaction mixture was quenched with saturated aq. $\mathrm{NaHCO}_{3}$ and extracted with DCM $(3 \times 50 \mathrm{~mL})$. The combined organic layers were washed 
with brine, dried over $\mathrm{Na}_{2} \mathrm{SO}_{4}$ and concentrated in vacuo. The crude residue was purified by flash chromatography $\left(\mathrm{SiO}_{2}, 20: 1\right.$ petroleum ether:EtOAc) to provide $\mathbf{S 1}(12.7 \mathrm{~g}, 83 \%)$ as a white solid.

Compound S1: mp: $131.3-134.5{ }^{\circ} \mathrm{C}$; TLC (petroleum ether:EtOAc, 5:1 v/v): $R_{f}=0.90$; $[\alpha]_{\mathrm{D}}^{25}+174.2\left(c 1.18, \mathrm{CHCl}_{3}\right) ;{ }^{1} \mathrm{H} \mathrm{NMR}\left(500 \mathrm{MHz}, \mathrm{CDCl}_{3}\right) \delta 5.66(\mathrm{dd}, J=6.0 \mathrm{~Hz}, 1 \mathrm{H})$, $5.50(\mathrm{dt}, J=7.0,2.5 \mathrm{~Hz}, 1 \mathrm{H}), 5.41(\mathrm{~d}, J=5.5 \mathrm{~Hz}, 1 \mathrm{H}), 5.24(\mathrm{dd}, J=15.5,7.5 \mathrm{~Hz}, 1 \mathrm{H})$, $5.17(\mathrm{dd}, J=15.5,7.5 \mathrm{~Hz}, 1 \mathrm{H}), 3.55(\mathrm{tt}, J=11.0,5.0 \mathrm{~Hz}, 1 \mathrm{H}), 2.49$ (t, $J=12.0 \mathrm{~Hz}, 1 \mathrm{H})$, $2.35(\mathrm{dd}, J=17.5,7.0 \mathrm{~Hz}, 1 \mathrm{H}), 2.28-2.20(\mathrm{~m}, 2 \mathrm{H}), 2.17(\mathrm{~d}, J=17.5 \mathrm{~Hz}, 1 \mathrm{H}), 2.10-$ $1.97(\mathrm{~m}, 1 \mathrm{H}), 1.91-1.79(\mathrm{~m}, 3 \mathrm{H}), 1.78-1.71(\mathrm{~m}, 2 \mathrm{H}), 1.71-1.65(\mathrm{~m}, 1 \mathrm{H}), 1.49(\mathrm{td}, J=$ 13.0, 6.0 Hz, 2H), $1.44-1.28(\mathrm{~m}, 3 \mathrm{H}), 1.24(\mathrm{~s}, 3 \mathrm{H}), 1.02(\mathrm{~d}, J=6.5 \mathrm{~Hz}, 3 \mathrm{H}), 0.92(\mathrm{~d}, J=$ $7.0 \mathrm{~Hz}, 3 \mathrm{H}), 0.89$ (s, 9H), 0.84 (d, $J=7.0 \mathrm{~Hz}, 3 \mathrm{H}), 0.82$ (d, $J=7.0 \mathrm{~Hz}, 3 \mathrm{H}), 0.58$ (s, 3H), 0.05 (s, 6H); ${ }^{13} \mathrm{C}$ NMR $\left(126 \mathrm{MHz}, \mathrm{CDCl}_{3}\right) \delta 144.5,142.4,135.7,135.4,132.2,122.4$, 118.0, 115.9, 73.3, 56.3, 51.2, 43.1, 42.9, 42.3, 42.3, 40.6, 39.6, 38.7, 33.2, 32.9, 30.6, 29.1, 26.1, 23.0, 20.8, 20.1, 19.8, 18.4, 17.7, 11.7, -4.5, -4.5; IR (KBr): $v_{\max }=3031$, 2956, 2928, 2855, 1588, 1459, 1379, 1256, 1090, 973, $836 \mathrm{~cm}^{-1}$; HRMS (DART, $\mathrm{m} / \mathrm{z}$ ): calcd for $\mathrm{C}_{34} \mathrm{H}_{57} \mathrm{OSi}[\mathrm{M}+\mathrm{H}]^{+}$509.4173, found 509.4177.

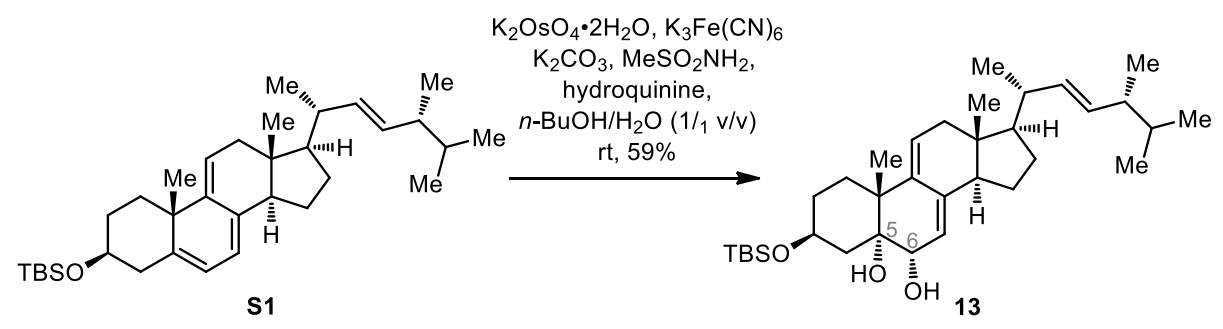

To a solution of $\mathbf{S 1}(2.0 \mathrm{~g}, 3.9 \mathrm{mmol}, 1.0$ equiv) in $200 \mathrm{~mL} n$-BuOH and $200 \mathrm{~mL}$ deionized $\mathrm{H}_{2} \mathrm{O}$ was sequentially added $\mathrm{K}_{3} \mathrm{Fe}(\mathrm{CN})_{6}(12.9 \mathrm{~g}$, $39.3 \mathrm{mmol}, 10.0$ equiv), $\mathrm{K}_{2} \mathrm{CO}_{3}\left(5.4 \mathrm{~g}, 39.3 \mathrm{mmol}, 10.0\right.$ equiv), $\mathrm{MeSO}_{2} \mathrm{NH}_{2}$ (373.8 mg, $3.9 \mathrm{mmol}, 1.0$ equiv), $\mathrm{K}_{2} \mathrm{OsO}_{4} \cdot 2 \mathrm{H}_{2} \mathrm{O}$ (434.8 mg, $1.2 \mathrm{mmol}, 0.3$ equiv) and hydroquinine (2.6 g, $7.9 \mathrm{mmol}, 2.0$ equiv) at room temperature. After stirred for 2 min under ultrasound to enhance the rate of dissolution, the reaction mixture was stirred at room temperature for $24 \mathrm{~h}$. The reaction was then quenched with saturated aq. $\mathrm{Na}_{2} \mathrm{~S}_{2} \mathrm{O}_{3}(30 \mathrm{~mL})$ and extracted with $\mathrm{DCM}(3 \times 100$ 
$\mathrm{mL}$ ). The combined organic layers were washed with brine, dried over $\mathrm{Na}_{2} \mathrm{SO}_{4}$ and concentrated in vacuo. The crude residue was purified by flash chromatography $\left(\mathrm{SiO}_{2}\right.$, $42: 1 \rightarrow 20: 1$ petroleum ether:EtOAc) to provide $\mathbf{1 3}$ (1.26 g, 59\% yield) as a white solid.

Compound 13: mp: $208.5-210.7^{\circ} \mathrm{C}$; TLC (petroleum ether:EtOAc, 10:1 v/v): $R_{f}=0.30$; $[\alpha]_{\mathrm{D}}^{25}+58.2\left(c 0.89, \mathrm{CHCl}_{3}\right) ;{ }^{1} \mathrm{H} \mathrm{NMR}\left(400 \mathrm{MHz}, \mathrm{CDCl}_{3}\right) \delta 5.66(\mathrm{~d}, J=6.8 \mathrm{~Hz}, 1 \mathrm{H}), 5.24$ (dd, $J=15.2,7.2 \mathrm{~Hz}, 1 \mathrm{H}), 5.15(\mathrm{dd}, J=15.2,8.0 \mathrm{~Hz}, 1 \mathrm{H}), 5.16-5.14(\mathrm{~m}, 1 \mathrm{H}), 4.05-$ 3.89 (m, 2H), 2.34 (dd, $J=17.6,6.8 \mathrm{~Hz}, 1 \mathrm{H}), 2.31-2.25(\mathrm{~m}, 1 \mathrm{H}), 2.22(\mathrm{~d}, J=10.4 \mathrm{~Hz}$, $1 \mathrm{H}), 2.21-2.11(\mathrm{~m}, 2 \mathrm{H}), 2.10-1.97(\mathrm{~m}, 1 \mathrm{H}), 1.90-1.73(\mathrm{~m}, 5 \mathrm{H}), 1.70-1.55(\mathrm{~m}, 2 \mathrm{H})$, $1.53-1.43(\mathrm{~m}, 2 \mathrm{H}), 1.42-1.28(\mathrm{~m}, 4 \mathrm{H}), 1.09$ (s, 3H), 1.01 (d, $J=6.8 \mathrm{~Hz}, 3 \mathrm{H}), 0.92(\mathrm{~d}, J$ $=7.2 \mathrm{~Hz}, 3 \mathrm{H}), 0.89(\mathrm{~s}, 9 \mathrm{H}), 0.84(\mathrm{~d}, J=6.8 \mathrm{~Hz}, 3 \mathrm{H}), 0.82(\mathrm{~d}, J=6.4 \mathrm{~Hz}, 3 \mathrm{H}), 0.54(\mathrm{~s}$, 3H), 0.07 (s, 6H); $\left.{ }^{13} \mathrm{C} \mathrm{NMR} \mathrm{(126} \mathrm{MHz,} \mathrm{CDCl}_{3}\right) \delta 139.5,137.1,135.4,132.4,125.0$, 121.6, 76.3, 70.6, 67.9, 56.1, 51.3, 42.9, 42.5, 42.3, 41.2, 40.5, 37.9, 33.2, 31.4, 30.3, 29.0, $26.1,24.4,23.2,20.8,20.1,19.8,18.3,17.7,11.5,-4.4,-4.5 ; \mathrm{IR}(\mathrm{KBr}): v_{\max }=3450$, 2956, 2870, 1460, 1368, 1249, 1106, 922, 836, $775 \mathrm{~cm}^{-1}$; HRMS (ESI, $\mathrm{m} / \mathrm{z}$ ): calcd for $\mathrm{C}_{34} \mathrm{H}_{58} \mathrm{NaO}_{3} \mathrm{Si}[\mathrm{M}+\mathrm{Na}]^{+}$565.4047, found 565.4046.

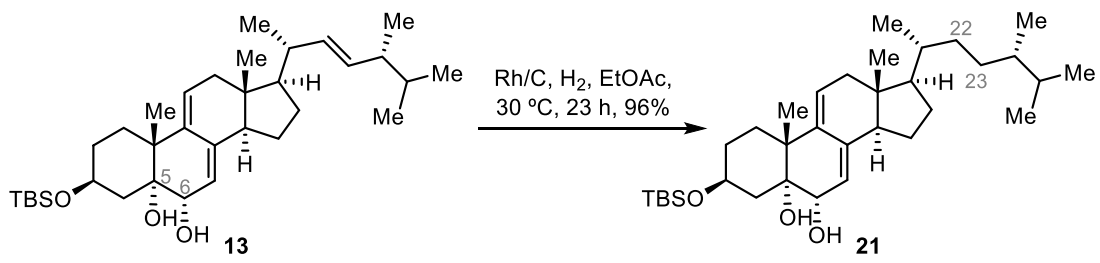

To a solution of 13 (1.63 g, $3.0 \mathrm{mmol}, 1.0$ equiv) in $160 \mathrm{~mL}$ EtOAc was added 10\% Rh/C $(0.49 \mathrm{~g}, 30 \mathrm{wt} \%)$ at room temperature. The reaction mixture was stirred at $30{ }^{\circ} \mathrm{C}$ under 1 atm $\mathrm{H}_{2}$ for $23 \mathrm{~h}$ before it was filtered through a short plug of Celite. The filter cake was washed with EtOAc $(30 \mathrm{~mL})$ and the filtrate was concentrated under vacuum. The crude residue was purified by flash chromatography $\left(\mathrm{SiO}_{2}, 5: 1\right.$ petroleum ether:EtOAc) to provide 21 (1.57 g, 96\%) as a white solid.

Compound 21: mp: $200.3-202.3{ }^{\circ} \mathrm{C}$; TLC (petroleum ether:EtOAc, 10:1 v/v): $R_{f}=0.25$; $[\alpha]_{\mathrm{D}}^{25}+66.2\left(c 0.81, \mathrm{CHCl}_{3}\right) ;{ }^{1} \mathrm{H} \mathrm{NMR}\left(400 \mathrm{MHz}, \mathrm{CDCl}_{3}\right) \delta 5.66(\mathrm{~d}, J=6.4 \mathrm{~Hz}, 1 \mathrm{H}), 5.16$ 
(brs, 1H), $4.07-3.86(\mathrm{~m}, 2 \mathrm{H}), 2.35(\mathrm{dd}, J=17.6,6.8 \mathrm{~Hz}, 1 \mathrm{H}), 2.31-2.09(\mathrm{~m}, 4 \mathrm{H}), 2.05$ - $1 . .92(\mathrm{~m}, 1 \mathrm{H}), 1.90-1.72(\mathrm{~m}, 3 \mathrm{H}), 1.69-1.54(\mathrm{~m}, 3 \mathrm{H}), 1.51-1.30(\mathrm{~m}, 8 \mathrm{H}), 1.26-$ $1.16(\mathrm{~m}, 1 \mathrm{H}), 1.09$ (s, 3H), $0.99-0.94(\mathrm{~m}, 2 \mathrm{H}), 0.91(\mathrm{~d}, J=6.0 \mathrm{~Hz}, 3 \mathrm{H}), 0.89$ (s, 9H), $0.86(\mathrm{~d}, J=6.8 \mathrm{~Hz}, 3 \mathrm{H}), 0.784(\mathrm{~d}, J=6.8 \mathrm{~Hz}, 3 \mathrm{H}), 0.780(\mathrm{~d}, J=6.8 \mathrm{~Hz}, 3 \mathrm{H}), 0.52(\mathrm{~s}, 3 \mathrm{H})$, 0.07 (s, 6H); ${ }^{13} \mathrm{C}$ NMR $\left(101 \mathrm{MHz}, \mathrm{CDCl}_{3}\right) \delta 139.5,137.2,125.1,121.5,76.4,70.7,67.9$, 56.2, 51.2, 42.7, 42.5, 41.2, 39.2, 38.0, 36.4, 33.7, 31.6, 31.4, 30.8, 30.3, 28.5, 26.1, 24.4, 23.2, 20.7, 18.8, 18.4, 17.7, 15.6, 11.4, -4.4, -4.5; IR (KBr): $v_{\max }=2957,2929,2864$, 1467, 1376, 1259, 994, 808, $775 \mathrm{~cm}^{-1}$; HRMS (ESI, $\mathrm{m} / z$ ): calcd for $\mathrm{C}_{34} \mathrm{H}_{60} \mathrm{NaO}_{3} \mathrm{Si}$ $[\mathrm{M}+\mathrm{Na}]^{+}$567.4204, found 567.4206.

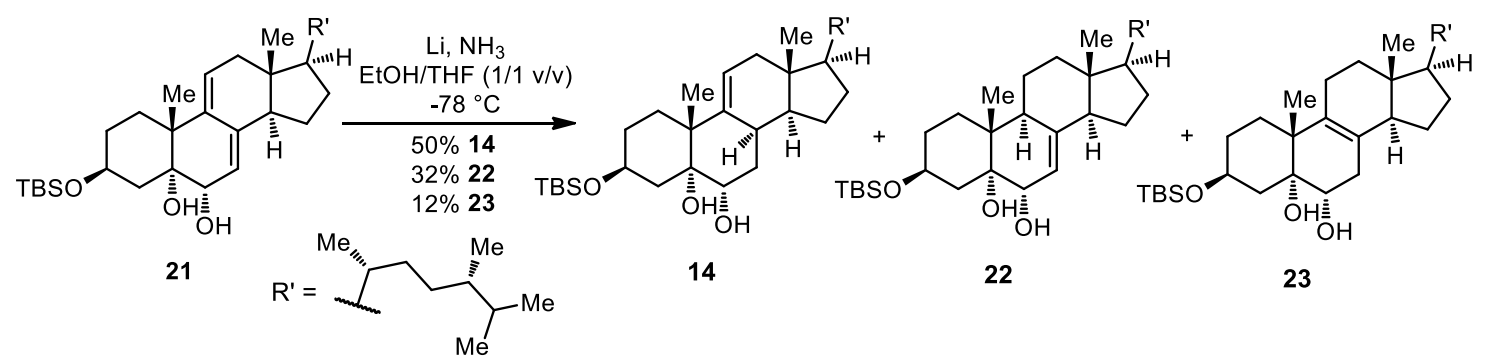

Ammonia $(184 \mathrm{~mL})$ was condensed into a three-neck round-bottom flask equipped with a stir bar at $-78{ }^{\circ} \mathrm{C}$. Under argon, 21 (1.3 g, $2.4 \mathrm{mmol}, 1.0$ equiv), anhydrous THF (184 mL) and anhydrous EtOH $(184 \mathrm{~mL})$ were sequentially added to this flask. Li wire (165.9 $\mathrm{mg} \times 8,23.9 \mathrm{mmol} \times 8,10$ equiv $\times 8$ ) was added in eight portions every $30 \mathrm{~min}$ to keep the solution dark yellow. After the completion of the addition of the Li wire (4 $\mathrm{h}$ in total), the reaction was quenched with $\mathrm{MeOH}$ at $-78{ }^{\circ} \mathrm{C}$ and warmed to room temperature to remove volatile $\mathrm{NH}_{3}$. After the organic solvent was removed by evaporation, the residue was extracted with brine $(100 \mathrm{~mL})$ and DCM $(3 \times 100 \mathrm{~mL})$. The combined organic layers were dried over anhydrous $\mathrm{Na}_{2} \mathrm{SO}_{4}$ and concentrated in vacuo. The crude residue was purified by flash chromatography $\left(\mathrm{SiO}_{2}, 50: 1 \rightarrow 40: 1 \rightarrow 20: 1\right.$ petroleum ether:EtOAc) to provide 14 (647.9 $\mathrm{mg}, 50 \%), \mathbf{2 2}(410.0 \mathrm{mg}, 32 \%)$, and $\mathbf{2 3}(153.1 \mathrm{mg}, 12 \%)$ as white solids.

Compound 14: mp: $222.7-224.0^{\circ} \mathrm{C}$; TLC (petroleum ether:EtOAc, 9:1 v/v): $R_{f}=0.53$; 
$[\alpha]_{\mathrm{D}}^{25}+68.7\left(c\right.$ 0.25, MeOH); ${ }^{1} \mathrm{H} \mathrm{NMR}\left(500 \mathrm{MHz}, \mathrm{CDCl}_{3}\right) \delta 5.48(\mathrm{dt}, J=7.5,2.5 \mathrm{~Hz}, 1 \mathrm{H})$, $4.02(\mathrm{tt}, J=11.0,5.0 \mathrm{~Hz}, 1 \mathrm{H}), 3.71(\mathrm{~d}, J=7.5 \mathrm{~Hz}, 1 \mathrm{H}), 3.35-3.21(\mathrm{~m}, 1 \mathrm{H}), 2.40(\mathrm{~s}, 1 \mathrm{H})$, $2.21(\mathrm{dd}, J=16.5,7.5 \mathrm{~Hz}, 1 \mathrm{H}), 2.14-2.00(\mathrm{~m}, 1 \mathrm{H}), 2.05(\mathrm{td}, J=14.5,8.0 \mathrm{~Hz}, 1 \mathrm{H}), 1.97$ $(\mathrm{ddd}, J=13.0,5.0,1.5 \mathrm{~Hz}, 1 \mathrm{H}), 1.94-1.86(\mathrm{~m}, 2 \mathrm{H}), 1.86-1.70(\mathrm{~m}, 4 \mathrm{H}), 1.66-1.56(\mathrm{~m}$, $3 \mathrm{H}), 1.55-1.46(\mathrm{~m}, 3 \mathrm{H}), 1.45-1.26(\mathrm{~m}, 5 \mathrm{H}), 1.23-1.17(\mathrm{~m}, 1 \mathrm{H}), 1.14(\mathrm{~s}, 3 \mathrm{H}), 0.94(\mathrm{t}$, $J=8.0 \mathrm{~Hz}, 2 \mathrm{H}), 0.90(\mathrm{~d}, J=5.5 \mathrm{~Hz}, 3 \mathrm{H}), 0.88(\mathrm{~s}, 9 \mathrm{H}), 0.85(\mathrm{~d}, J=7.0 \mathrm{~Hz}, 3 \mathrm{H}), 0.78(\mathrm{~d}, J$ $=7.0 \mathrm{~Hz}, 3 \mathrm{H}), 0.77(\mathrm{~d}, J=6.5 \mathrm{~Hz}, 3 \mathrm{H}), 0.63(\mathrm{~s}, 3 \mathrm{H}), 0.06(\mathrm{~s}, 3 \mathrm{H}), 0.05(\mathrm{~s}, 3 \mathrm{H}) ;{ }^{13} \mathrm{C} \mathrm{NMR}$ $\left(126 \mathrm{MHz}, \mathrm{CDCl}_{3}\right) \delta 146.6,119.7,77.0,69.9,68.3,55.7,48.2,42.0,41.7,41.4,40.6$, 39.2, 36.2, 33.8, 33.7, 31.9, 31.9, 31.6, 31.5, 30.8, 28.3, 27.3, 26.1, 22.8, 20.7, 18.7, 18.4, 17.7, 15.6, 12.4, -4.4, -4.5; IR (KBr): $v_{\max }=3416,2954,2871,1463,1367,1255,1102$, 837, $774 \mathrm{~cm}^{-1}$; HRMS (ESI, m/z): calcd for $\mathrm{C}_{34} \mathrm{H}_{62} \mathrm{NaO}_{3} \mathrm{Si}[\mathrm{M}+\mathrm{Na}]^{+}$569.4360, found 569.4369.

Compound 22: mp: $216.7-217.3{ }^{\circ} \mathrm{C}$; TLC (petroleum ether:EtOAc, 9:1 v/v): $R_{f}=0.47$; $[\alpha]_{\mathrm{D}}^{25}+15.6(c$ 0.18, MeOH)$) ;{ }^{1} \mathrm{H} \mathrm{NMR}\left(400 \mathrm{MHz}, \mathrm{CDCl}_{3}\right) \delta 5.01(\mathrm{q}, J=2.0 \mathrm{~Hz}, 1 \mathrm{H}), 4.03$ - $3.88(\mathrm{~m}, 2 \mathrm{H}), 2.14-2.01(\mathrm{~m}, 2 \mathrm{H}), 1.97-1.83(\mathrm{~m}, 4 \mathrm{H}), 1.79-1.68(\mathrm{~m}, 2 \mathrm{H}), 1.62-$ $1.54(\mathrm{~m}, 5 \mathrm{H}), 1.53-1.43(\mathrm{~m}, 5 \mathrm{H}), 1.43-1.31(\mathrm{~m}, 4 \mathrm{H}), 1.31-1.26(\mathrm{~m}, 2 \mathrm{H}), 1.25-1.16$ (m, 2H), $0.96(\mathrm{~s}, 3 \mathrm{H}), 0.92(\mathrm{~d}, J=6.4 \mathrm{~Hz}, 3 \mathrm{H}), 0.89(\mathrm{~s}, 9 \mathrm{H}), 0.85$ (d, $J=6.8 \mathrm{~Hz}, 3 \mathrm{H})$, $0.78(\mathrm{~d}, J=6.8 \mathrm{~Hz}, 3 \mathrm{H}), 0.77(\mathrm{~d}, J=6.4 \mathrm{~Hz}, 3 \mathrm{H}), 0.54(\mathrm{~s}, 3 \mathrm{H}), 0.07(\mathrm{~s}, 6 \mathrm{H}) ;{ }^{13} \mathrm{C} \mathrm{NMR}$ $\left(126 \mathrm{MHz}, \mathrm{CDCl}_{3}\right) \delta 143.1,120.3,76.2,70.6,68.2,56.1,54.8,44.0,43.4,39.5,39.3$, $39.2,38.6,36.7,33.7,31.8,31.6,31.3,30.8,28.0,26.1,22.9,21.5,20.7,19.1,18.3,18.0$, $17.7,15.6,12.1,-4.4,-4.5 ; \mathrm{IR}(\mathrm{KBr}): v_{\max }=3445,2954,2864,1463,1376,1250,1098$, 872, 835, $774 \mathrm{~cm}^{-1}$; HRMS (ESI, m/z): calcd for $\mathrm{C}_{34} \mathrm{H}_{62} \mathrm{NaO}_{3} \mathrm{Si}[\mathrm{M}+\mathrm{Na}]^{+}$569.4360, found 569.4358 .

Compound 23: mp: $189.1-191.0{ }^{\circ} \mathrm{C}$; TLC (petroleum ether:EtOAc, 9:1 v/v): $R_{f}=0.38$; $[\alpha]_{\mathrm{D}}^{25}+5.3(c \mathrm{0} .24, \mathrm{MeOH}) ;{ }^{1} \mathrm{H} \mathrm{NMR}\left(400 \mathrm{MHz}, \mathrm{CDCl}_{3}\right) \delta 4.01(\mathrm{tt}, J=11.2,5.2 \mathrm{~Hz}, 1 \mathrm{H})$, $3.81(\mathrm{dt}, J=10.0,8.0 \mathrm{~Hz}, 1 \mathrm{H}), 2.55(\mathrm{dd}, J=18.8,7.6 \mathrm{~Hz}, 1 \mathrm{H}), 2.22(\mathrm{dd}, J=13.2,5.2 \mathrm{~Hz}$, 1H), $2.17-2.06(\mathrm{~m}, 1 \mathrm{H}), 2.06-1.97(\mathrm{~m}, 3 \mathrm{H}), 1.96-1.70(\mathrm{~m}, 5 \mathrm{H}), 1.70-1.52(\mathrm{~m}, 5 \mathrm{H})$, 
$1.52-1.25(\mathrm{~m}, 9 \mathrm{H}), 1.25-1.15(\mathrm{~m}, 2 \mathrm{H}), 1.13(\mathrm{~s}, 3 \mathrm{H}), 0.92(\mathrm{~d}, J=6.8 \mathrm{~Hz}, 3 \mathrm{H}), 0.89(\mathrm{~s}$, $9 \mathrm{H}), 0.85(\mathrm{~d}, J=6.8 \mathrm{~Hz}, 3 \mathrm{H}), 0.78(\mathrm{~d}, J=6.8 \mathrm{~Hz}, 3 \mathrm{H}), 0.77$ (d, $J=6.8 \mathrm{~Hz}, 3 \mathrm{H}), 0.59$ (s, 3H), 0.06 (s, 6H); ${ }^{13} \mathrm{C}$ NMR (101 MHz, $\left.\mathrm{CDCl}_{3}\right) \delta$ 132.6, 129.3, 76.7, 68.6, 68.0, 54.6, 51.4, 42.4, 42.1, 39.2, 36.8, 36.7, 36.6, 35.0, 33.7, 31.6, 31.3, 30.8, 30.0, 28.8, 26.1, 23.5, 23.3, 23.2, 20.7, 19.0, 18.4, 17.7, 15.6, 11.1, -4.4, -4.5; IR (KBr): $v_{\max }=3417,2956$, 2861, 1463, 1376, 1257, 1089, 874, $836 \mathrm{~cm}^{-1}$; HRMS (ESI, $\mathrm{m} / z$ ): calcd for $\mathrm{C}_{34} \mathrm{H}_{62} \mathrm{NaO}_{3} \mathrm{Si}$ $[\mathrm{M}+\mathrm{Na}]^{+}$569.4360, found 569.4365 .

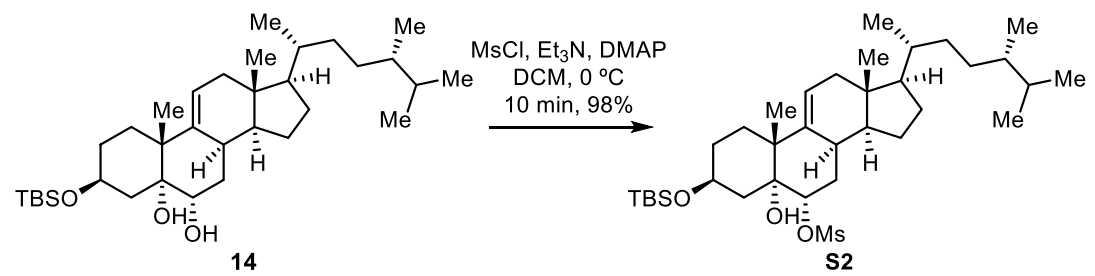

To a solution of 14 (1.26 g, $2.3 \mathrm{mmol}, 1.0$ equiv) and DMAP (28.2 mg, $0.23 \mathrm{mmol}, 0.1$ equiv) in $115 \mathrm{~mL}$ DCM was added $\mathrm{Et}_{3} \mathrm{~N}(1.28 \mathrm{~mL}, 9.2 \mathrm{mmol}, 4.0$ equiv) and $\mathrm{MsCl}$ (357 $\mu \mathrm{L}, 4.6 \mathrm{mmol}, 2.0$ equiv) at $0{ }^{\circ} \mathrm{C}$. After stirred at $0{ }^{\circ} \mathrm{C}$ for $10 \mathrm{~min}$, the reaction mixture was quenched with saturated aq. $\mathrm{NaHCO}_{3}(10 \mathrm{~mL})$ and extracted with $\mathrm{DCM}(3 \times 30 \mathrm{~mL})$. The combined organic layers were washed with brine, dried over $\mathrm{Na}_{2} \mathrm{SO}_{4}$ and concentrated in vacuo. The crude residue was purified by flash chromatography $\left(\mathrm{SiO}_{2}\right.$, 15:1 petroleum ether:EtOAc) to provide $\mathbf{S 2}$ (1.42 g, 98\%) as a white solid.

Compound S2: mp: $143.1-155.0{ }^{\circ} \mathrm{C}$; TLC (petroleum ether:EtOAc, 9:1 v/v): $R_{f}=0.32$; $[\alpha]_{\mathrm{D}}^{25}+73.1(c 0.27, \mathrm{MeOH}) ;{ }^{1} \mathrm{H} \mathrm{NMR}\left(500 \mathrm{MHz}, \mathrm{CDCl}_{3}\right) \delta 5.48(\mathrm{dt}, J=8.0,2.0 \mathrm{~Hz}, 1 \mathrm{H})$, $4.68(\mathrm{~d}, J=8.5 \mathrm{~Hz}, 1 \mathrm{H}), 4.01(\mathrm{tt}, J=11.0,5.0 \mathrm{~Hz}, 1 \mathrm{H}), 3.64-3.43(\mathrm{~m}, 1 \mathrm{H}), 3.06(\mathrm{~s}, 3 \mathrm{H})$, $2.31-2.13(\mathrm{~m}, 2 \mathrm{H}), 1.98-1.86(\mathrm{~m}, 6 \mathrm{H}), 1.85-1.76(\mathrm{~m}, 2 \mathrm{H}), 1.71(\mathrm{dt}, J=14.0,4.0 \mathrm{~Hz}$, 1H), $1.58-1.49(\mathrm{~m}, 3 \mathrm{H}), 1.47-1.24(\mathrm{~m}, 6 \mathrm{H}), 1.23-1.18(\mathrm{~m}, 1 \mathrm{H}), 1.17$ (s, 3H), $1.12(\mathrm{q}$, $J=9.5 \mathrm{~Hz}, 1 \mathrm{H}), 0.94(\mathrm{t}, J=7.5 \mathrm{~Hz}, 2 \mathrm{H}), 0.89(\mathrm{~d}, J=5.0 \mathrm{~Hz}, 3 \mathrm{H}), 0.88(\mathrm{~s}, 9 \mathrm{H}), 0.85$ (d, $J$ $=6.5 \mathrm{~Hz}, 3 \mathrm{H}), 0.78(\mathrm{~d}, J=6.5 \mathrm{~Hz}, 3 \mathrm{H}), 0.77(\mathrm{~d}, J=7.0 \mathrm{~Hz}, 3 \mathrm{H}), 0.63(\mathrm{~s}, 3 \mathrm{H}), 0.06(\mathrm{~s}$, $3 \mathrm{H}), 0.05(\mathrm{~s}, 3 \mathrm{H}) ;{ }^{13} \mathrm{C} \mathrm{NMR}\left(126 \mathrm{MHz}, \mathrm{CDCl}_{3}\right) \delta$ 145.4, 120.2, 82.2, 77.0, 67.9, 55.6, 
47.8, 42.0, 41.6, 41.2, 40.9, 39.2, 38.9, 36.2, 33.6, 31.61, 31.58, 31.4, 31.3 (2C), 30.8, 28.2, 27.4, 26.1, 22.7, 20.7, 18.6, 18.3, 17.7, 15.6, 12.5, -4.3, -4.4; IR (KBr): $v_{\max }=3541$, 2955, 2871, 1466, 1366, 1251, 1102, 963, $836 \mathrm{~cm}^{-1}$; HRMS (ESI, $\mathrm{m} / \mathrm{z}$ ): calcd for $\mathrm{C}_{35} \mathrm{H}_{64} \mathrm{NaO}_{5} \mathrm{Si}[\mathrm{M}+\mathrm{Na}]^{+}$647.4136, found 647.4144.

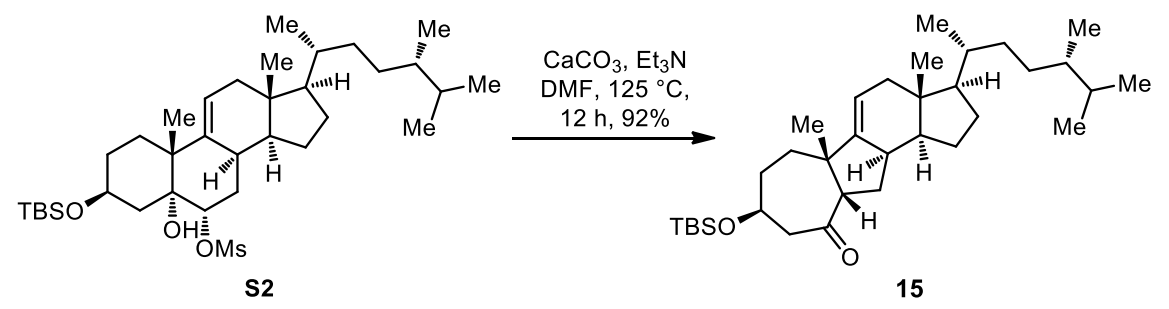

To a solution of $\mathbf{S 2}\left(1.42 \mathrm{~g}, 2.29 \mathrm{mmol}, 1.0\right.$ equiv) and $\mathrm{CaCO}_{3}(1.03 \mathrm{~g}, 10.3 \mathrm{mmol}, 4.5$ equiv) in $114 \mathrm{~mL}$ DMF was added $\mathrm{Et}_{3} \mathrm{~N}$ (1.9 mL, $13.7 \mathrm{mmol}, 6.0$ equiv) at room temperature. After stirred at $125{ }^{\circ} \mathrm{C}$ for $12 \mathrm{~h}$, the reaction mixture was quenched with saturated aq. $\mathrm{NaHCO}_{3}(30 \mathrm{~mL})$ and extracted with petroleum ether $(4 \times 30 \mathrm{~mL})$. The combined organic layers were washed with brine, dried over $\mathrm{Na}_{2} \mathrm{SO}_{4}$ and concentrated in vacuo. The crude residue was purified by flash chromatography $\left(\mathrm{SiO}_{2}, 20: 1\right.$ petroleum ether:EtOAc) to provide $\mathbf{1 5}(1.11 \mathrm{~g}, 92 \%)$ as a white solid.

Compound 15: mp: $149.5-150.8{ }^{\circ} \mathrm{C}$; TLC (petroleum ether:EtOAc, 9:1 v/v): $R_{f}=0.90$; $[\alpha]_{\mathrm{D}}^{25}+42.6\left(c 0.65, \mathrm{CHCl}_{3}\right) ;{ }^{1} \mathrm{H} \mathrm{NMR}\left(500 \mathrm{MHz}, \mathrm{CDCl}_{3}\right) \delta 5.23(\mathrm{dt}, J=7.5,2.5 \mathrm{~Hz}, 1 \mathrm{H})$, 3.70 (tt, $J=10.5,4.5 \mathrm{~Hz}, 1 \mathrm{H}), 2.80$ (t, $J=10.0 \mathrm{~Hz}, 1 \mathrm{H}), 2.74(\mathrm{~d}, J=8.5 \mathrm{~Hz}, 1 \mathrm{H}), 2.65-$ $2.45(\mathrm{~m}, 2 \mathrm{H}), 2.24(\mathrm{dd}, J=16.0,7.5 \mathrm{~Hz}, 1 \mathrm{H}), 2.12(\mathrm{dd}, J=12.0,7.0 \mathrm{~Hz}, 1 \mathrm{H}), 1.98-1.85$ (m, 2H), $1.84-1.76(\mathrm{~m}, 2 \mathrm{H}), 1.76-1.64(\mathrm{~m}, 2 \mathrm{H}), 1.48(\mathrm{~s}, 3 \mathrm{H}), 1.43-1.35(\mathrm{~m}, 3 \mathrm{H}), 1.35$ $-1.26(\mathrm{~m}, 5 \mathrm{H}), 1.24-1.16(\mathrm{~m}, 2 \mathrm{H}), 1.10(\mathrm{q}, J=9.5 \mathrm{~Hz}, 1 \mathrm{H}), 0.94(\mathrm{t}, J=7.5 \mathrm{~Hz}, 2 \mathrm{H})$, $0.89(\mathrm{~d}, J=6.5 \mathrm{~Hz}, 3 \mathrm{H}), 0.88(\mathrm{~s}, 9 \mathrm{H}), 0.85(\mathrm{~d}, J=7.0 \mathrm{~Hz}, 3 \mathrm{H}), 0.78(\mathrm{~d}, J=6.5 \mathrm{~Hz}, 3 \mathrm{H})$, $0.77(\mathrm{~d}, J=6.5 \mathrm{~Hz}, 3 \mathrm{H}), 0.61(\mathrm{~s}, 3 \mathrm{H}), 0.07(\mathrm{~s}, 6 \mathrm{H}) ;{ }^{13} \mathrm{C} \mathrm{NMR}\left(126 \mathrm{MHz}, \mathrm{CDCl}_{3}\right) \delta 209.4$, 154.5, 113.5, 71.0, 61.0, 55.1, 54.3, 47.6, 44.4, 42.2, 40.2, 40.1, 39.2, 36.0 (2C), 34.8, 33.7, 31.6, 30.8, 28.5, 28.3, 26.2, 26.0, 23.4, 20.7, 18.7, 18.3, 17.7, 15.6, 12.7, -4.6, -4.7; IR (KBr): $v_{\max }=2957,2857,1699,1463,1376,1259,1089,836 \mathrm{~cm}^{-1}$; HRMS (ESI, $\left.m / z\right)$ : 
calcd for $\mathrm{C}_{34} \mathrm{H}_{60} \mathrm{NaO}_{2} \mathrm{Si}[\mathrm{M}+\mathrm{Na}]^{+}$551.4255, found 551.4260.
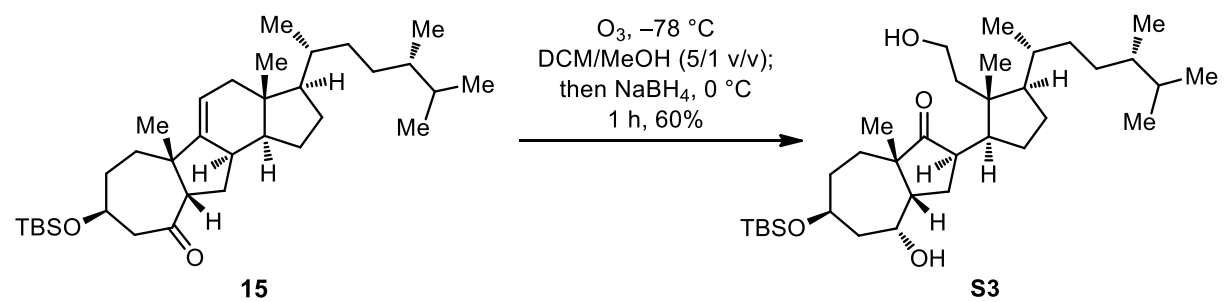

A solution of 15 (350 mg, $0.662 \mathrm{mmol}, 1.0$ equiv) in DCM/MeOH (40 mL, 5:1 v/v) at $-78{ }^{\circ} \mathrm{C}$ was purged with ozone for 1 min until the solution turned blue. The system was then purged with $\mathrm{N}_{2}$ for approximately $15 \mathrm{~min}$ until the blue color disappeared. After $\mathrm{NaBH}_{4}\left(50.1 \mathrm{mg}, 1.324 \mathrm{mmol}, 2.0\right.$ equiv) was added at $-78{ }^{\circ} \mathrm{C}$, the reaction mixture was warmed to $0{ }^{\circ} \mathrm{C}$ and stirred for $1 \mathrm{~h}$. Saturated aq. $\mathrm{NH}_{4} \mathrm{Cl}(10 \mathrm{~mL})$ was added to quench the reaction, and $\mathrm{MeOH}$ was removed under reduced pressure. The residue was extracted with brine $(15 \mathrm{~mL})$ and $\mathrm{DCM}(3 \times 15 \mathrm{~mL})$. The combined organic layers were washed with brine, dried over $\mathrm{Na}_{2} \mathrm{SO}_{4}$ and concentrated in vacuo. The crude residue was purified by flash chromatography $\left(\mathrm{SiO}_{2}, 6: 1\right.$ petroleum ether:EtOAc) to provide $\mathbf{S 3}$ (222.5 mg, $60 \%$ ) as colorless oil.

Compound S3: TLC (petroleum ether:EtOAc, 3:1 v/v): $R_{f}=0.23 ;[\alpha]_{\mathrm{D}}^{25}+39.7(c 0.64$, $\left.\mathrm{CHCl}_{3}\right) ;{ }^{1} \mathrm{H} \mathrm{NMR}\left(500 \mathrm{MHz}, \mathrm{CDCl}_{3}\right) \delta 4.20(\mathrm{~d}, J=7.5 \mathrm{~Hz}, 1 \mathrm{H}), 3.93-3.84(\mathrm{~m}, 1 \mathrm{H})$, $3.78-3.66(\mathrm{~m}, 2 \mathrm{H}), 2.5(\mathrm{q}, J=8.0 \mathrm{~Hz}, 1 \mathrm{H}), 2.14-2.04(\mathrm{~m}, 5 \mathrm{H}), 1.86-1.76(\mathrm{~m}, 2 \mathrm{H})$, $1.75-1.59(\mathrm{~m}, 8 \mathrm{H}), 1.58-1.50(\mathrm{~m}, 3 \mathrm{H}), 1.48-1.29(\mathrm{~m}, 6 \mathrm{H}), 1.24-1.15(\mathrm{~m}, 2 \mathrm{H}), 1.04$ (s, 3H), $0.96(\mathrm{~d}, J=6.0 \mathrm{~Hz}, 3 \mathrm{H}), 0.88(\mathrm{~s}, 9 \mathrm{H}), 0.84(\mathrm{~d}, J=7.0 \mathrm{~Hz}, 3 \mathrm{H}), 0.80(\mathrm{~s}, 3 \mathrm{H})$, $0.773(\mathrm{~d}, J=7.0 \mathrm{~Hz}, 3 \mathrm{H}), 0.765$ (d, $J=7.0 \mathrm{~Hz}, 3 \mathrm{H}), 0.044(\mathrm{~s}, 3 \mathrm{H}), 0.038(\mathrm{~s}, 3 \mathrm{H}) ;{ }^{13} \mathrm{C}$ NMR $\left(126 \mathrm{MHz} \mathrm{CDCl}_{3}\right) \delta 224.8,72.1,69.7,59.2,50.3,50.2,49.9,48.3,47.9,46.3,45.2$, $41.2,39.2,34.2,33.1,33.0,32.6,31.8,31.6,30.2,26.0,26.0,25.4,25.3,20.6,20.2$, 18.3, $17.8,17.4,15.6,-4.6,-4.7$; IR $(\mathrm{KBr}): v_{\max }=3411,2956,2871,1720,1462,1377,1257$, 1076, 836, $775 \mathrm{~cm}^{-1}$; HRMS (ESI, $\mathrm{m} / z$ ): calcd for $\mathrm{C}_{34} \mathrm{H}_{65} \mathrm{O}_{4} \mathrm{Si}[\mathrm{M}+\mathrm{H}]^{+}$565.4647, found 565.4646 . 


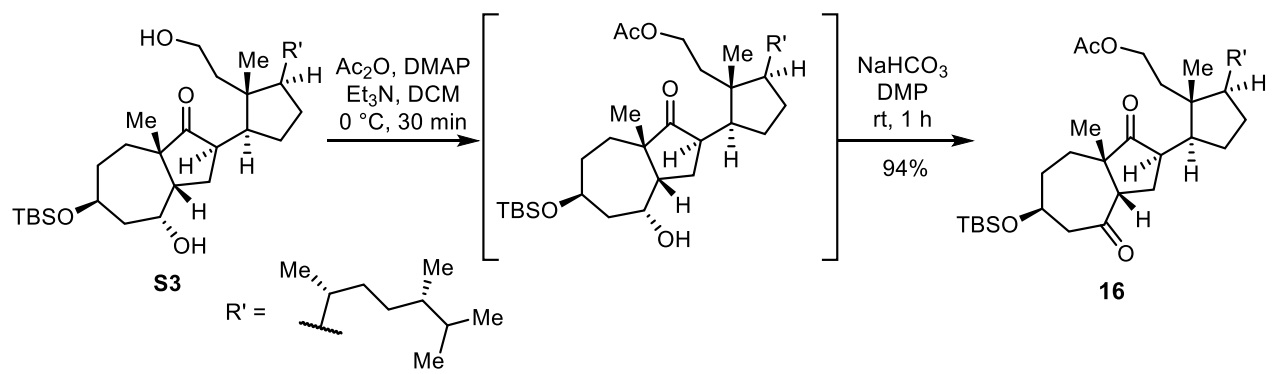

To a solution of $\mathbf{S 3}$ (308.0 mg, $0.394 \mathrm{mmol}, 1.0$ equiv) and DMAP (6.7 mg, $0.0546 \mathrm{mmol}$, 0.1 equiv) in $20 \mathrm{~mL}$ DCM was added $\mathrm{Et}_{3} \mathrm{~N}\left(228 \mu \mathrm{L}, 1.64 \mathrm{mmol}, 3.0\right.$ equiv) and $\mathrm{Ac}_{2} \mathrm{O}$ (62 $\mu \mathrm{L}, 0.655 \mathrm{mmol}, 1.2$ equiv) at $0{ }^{\circ} \mathrm{C}$. The reaction mixture was stirred at the same temperature for $30 \mathrm{~min}$. Then $\mathrm{NaHCO}_{3}(458.7 \mathrm{mg}, 5.46 \mathrm{mmol}, 10.0$ equiv) and Dess-Martin periodinane (DMP) (1.16 g, $2.73 \mathrm{mmol}, 5.0$ equiv) were added successively at $0{ }^{\circ} \mathrm{C}$ and stirred at room temperature for $1 \mathrm{~h}$. The reaction mixture was quenched with saturated with aq. $\mathrm{NaHCO}_{3}(15 \mathrm{~mL})$ and extracted with DCM $(3 \times 15 \mathrm{~mL})$. The combined organic layers were washed with brine, dried over $\mathrm{Na}_{2} \mathrm{SO}_{4}$ and concentrated in vacuo. The crude residue was purified by flash chromatography $\left(\mathrm{SiO}_{2}, 6: 1\right.$ petroleum ether:EtOAc) to provide 16 (315.0 $\mathrm{mg}, 94 \%)$ as colorless oil.

Compound 16: TLC (petroleum ether:EtOAc, 3:1 v/v): $R_{f}=0.90 ;[\alpha]_{\mathrm{D}}^{25}+33.3(c 0.75$, $\left.\mathrm{CHCl}_{3}\right) ;{ }^{1} \mathrm{H} \mathrm{NMR}\left(400 \mathrm{MHz}, \mathrm{CDCl}_{3}\right) \delta 4.21-4.01(\mathrm{~m}, 2 \mathrm{H}), 3.82(\mathrm{tt}, J=8.4,4.0 \mathrm{~Hz}, 1 \mathrm{H})$, $3.12(\mathrm{~d}, J=6.8 \mathrm{~Hz}, 1 \mathrm{H}), 2.74(\mathrm{dd}, J=11.6,10.0 \mathrm{~Hz}, 1 \mathrm{H}), 2.62(\mathrm{dd}, J=11.2,5.2 \mathrm{~Hz}, 1 \mathrm{H})$, $2.57(\mathrm{dd}, J=12.4,8.8 \mathrm{~Hz}, 1 \mathrm{H}), 2.40(\mathrm{dt}, J=12.4,8.0 \mathrm{~Hz}, 1 \mathrm{H}), 2.03(\mathrm{~s}, 3 \mathrm{H}), 2.09-1.97$ $(\mathrm{m}, 1 \mathrm{H}), 1.93-1.71(\mathrm{~m}, 7 \mathrm{H}), 1.70-1.61(\mathrm{~m}, 2 \mathrm{H}), 1.60-1.45(\mathrm{~m}, 3 \mathrm{H}), 1.45-1.35(\mathrm{~m}$, 5H), $1.23(\mathrm{~s}, 3 \mathrm{H}), 1.26-1.15(\mathrm{~m}, 2 \mathrm{H}), 0.97$ (d, J = 6.4 Hz, 3H), 0.87 (s, 9H), 0.86 (s, 3H), $0.85(\mathrm{~d}, J=7.6 \mathrm{~Hz}, 3 \mathrm{H}), 0.78(\mathrm{~d}, J=6.8 \mathrm{~Hz}, 3 \mathrm{H}), 0.77$ (d, $J=6.5 \mathrm{~Hz}, 3 \mathrm{H}), 0.06(\mathrm{~s}, 3 \mathrm{H})$, $0.05(\mathrm{~s}, 3 \mathrm{H}) ;{ }^{13} \mathrm{C}$ NMR $\left(126 \mathrm{MHz}, \mathrm{CDCl}_{3}\right) \delta 221.2,209.3,171.3,69.4,61.4,56.9,52.9$, 50.1, 49.8, 49.2, 47.1, 46.1, 39.2, 36.3, 34.0, 33.0, 32.8, 31.8, 31.7, 30.6, 27.5, 26.1, 25.9, $25.5,24.5,21.3,20.6,20.2,18.2,17.8,17.2,15.6,-4.7(2 \mathrm{C})$; IR (KBr): $v_{\max }=2957,2872$, 1740, 1705, 1463, 1367, 1252, 1080, $838 \mathrm{~cm}^{-1}$; HRMS (ESI, $\left.m / z\right)$ : calcd for $\mathrm{C}_{36} \mathrm{H}_{65} \mathrm{O}_{5} \mathrm{Si}$ $[\mathrm{M}+\mathrm{H}]^{+}$605.4596, found 605.4594 . 

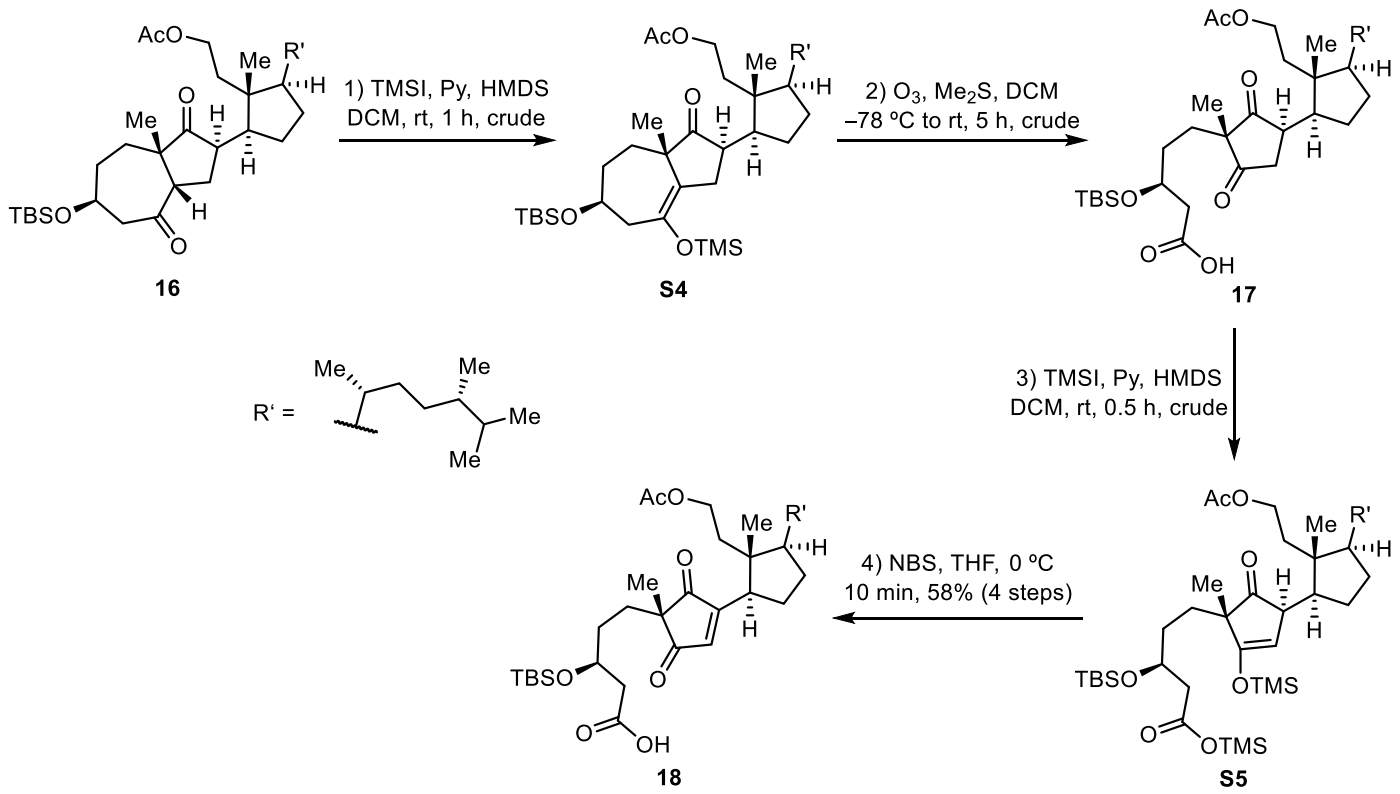

To a solution of 16 (127.0 mg, $0.21 \mathrm{mmol}, 1.0$ equiv) in $7 \mathrm{~mL}$ DCM was added HMDS (263.0 $\mu \mathrm{L}, 1.26 \mathrm{mmol}, 6.0$ equiv), pyridine $(51.0 \mu \mathrm{L}, 0.63 \mathrm{mmol}, 3.0$ equiv) and TMSI (135.0 $\mu \mathrm{L}, 0.95 \mathrm{mmol}, 4.5$ equiv) at $0{ }^{\circ} \mathrm{C}$ under argon, and the ice bath was removed. After the reaction mixture was stirred at room temperature for $0.5 \mathrm{~h}$, another portion of HMDS (263.0 $\mu \mathrm{L}, 1.26 \mathrm{mmol}, 6.0$ equiv), pyridine ( $5.01 \mu \mathrm{L}, 0.63 \mathrm{mmol}, 3.0$ equiv) and TMSI (135.0 $\mu \mathrm{L}, 0.95 \mathrm{mmol}, 4.5$ equiv) was added. The reaction mixture was stirred at room temperature for another $0.5 \mathrm{~h}$, quenched with sat. aq. $\mathrm{NaHCO}_{3}$ and extracted with DCM $(3 \times 10 \mathrm{~mL})$. The combined organic layers were dried over $\mathrm{Na}_{2} \mathrm{SO}_{4}$. Removal of the solvent under reduced pressure afforded the crude product $\mathbf{S 4}$, which was used in the next step without further purification.

A solution of crude silyl enol ether $\mathbf{S 4}$ obtained above in $7 \mathrm{~mL} \mathrm{DCM}$ at $-78{ }^{\circ} \mathrm{C}$ was purged with ozone until the solution turned blue. The system was then purged with $\mathrm{N}_{2}$ until the blue color disappeared. To this solution were added $\mathrm{Me}_{2} \mathrm{~S}(46.0 \mu \mathrm{L}, 0.63 \mathrm{mmol}$, 3.0 equiv) at $-78{ }^{\circ} \mathrm{C}$. The reaction mixture was warmed to room temperature and stirred for $5 \mathrm{~h}$. Removal of the solvent under reduced pressure afforded the crude product 17, which was used in the next step without further purification.

To a solution of crude 17 (127.0 mg, $0.21 \mathrm{mmol}, 1.0$ equiv) in $7 \mathrm{~mL} \mathrm{DCM}$ was added 
HMDS (263.0 $\mu \mathrm{L}, 1.26 \mathrm{mmol}, 6.0$ equiv), pyridine ( $51.0 \mu \mathrm{L}, 0.63 \mathrm{mmol}, 3.0$ equiv) and TMSI (135.0 $\mu \mathrm{L}, 0.95 \mathrm{mmol}, 4.5$ equiv) at $0{ }^{\circ} \mathrm{C}$ under argon. After the ice bath was removed, the reaction mixture was stirred at room temperature for $0.5 \mathrm{~h}$, quenched with sat. aq. $\mathrm{NaHCO}_{3}$ and extracted with DCM $(3 \times 10 \mathrm{~mL})$. The combined organic layers were dried over $\mathrm{Na}_{2} \mathrm{SO}_{4}$. Removal of the solvent under reduced pressure afforded the crude product $\mathbf{S 5}$, which was used in the next step without further purification.

To a solution of crude silyl enol ether $\mathbf{S 5}$ obtained above in $7 \mathrm{~mL}$ THF was added NBS (41.0 mg, $0.23 \mathrm{mmol}, 1.1$ equiv) at $0{ }^{\circ} \mathrm{C}$ under argon. The reaction mixture was stirred at $0{ }^{\circ} \mathrm{C}$ for 10 min, quenched with $\mathrm{H}_{2} \mathrm{O}$ and extracted with EtOAc $(3 \times 10 \mathrm{~mL})$. The combined organic layers were washed with brine and dried over $\mathrm{Na}_{2} \mathrm{SO}_{4}$. Removal of the solvent under reduced pressure afforded the crude product, which was purified by flash chromatography ( $\mathrm{SiO}_{2}, 50: 1 \rightarrow 4: 1 \rightarrow 3: 2$ petroleum ether:EtOAc) to provide 18 (78.0 mg, $58 \%$ ) as light yellow oil.

Compound 17: TLC (petroleum ether:EtOAc, 1:1 v/v): $R_{f}=0.2$; $[\alpha]_{\mathrm{D}}^{25}+34.6(c$ 1.10, $\left.\mathrm{CHCl}_{3}\right) ;{ }^{1} \mathrm{H} \mathrm{NMR}\left(400 \mathrm{MHz}, \mathrm{CDCl}_{3}\right) \delta 4.18-3.99(\mathrm{~m}, 3 \mathrm{H}), 3.04-2.84(\mathrm{~m}, 1 \mathrm{H}), 2.77-$ $2.62(\mathrm{~m}, 2 \mathrm{H}), 2.52-2.37(\mathrm{~m}, 2 \mathrm{H}), 2.03(\mathrm{~s}, 3 \mathrm{H}), 2.10-1.82(\mathrm{~m}, 4 \mathrm{H}), 1.81-1.70(\mathrm{~m}, 1 \mathrm{H})$, $1.70-1.28(\mathrm{~m}, 10 \mathrm{H}), 1.28-1.11(\mathrm{~m}, 2 \mathrm{H}), 1.08(\mathrm{~s}, 3 \mathrm{H}), 1.01-0.90(\mathrm{~m}, 2 \mathrm{H}), 0.98(\mathrm{~d}, J=$ $6.4 \mathrm{~Hz}, 3 \mathrm{H}), 0.86$ (s, 9H), 0.85 (d, $J=6.4 \mathrm{~Hz}, 3 \mathrm{H}), 0.82$ (s, 3H), $0.78(\mathrm{dd}, J=6.8 \mathrm{~Hz}, 3 \mathrm{H})$, $0.77(\mathrm{dd}, J=6.8 \mathrm{~Hz}, 3 \mathrm{H}), 0.05(\mathrm{~s}, 3 \mathrm{H}), 0.03(\mathrm{~s}, 3 \mathrm{H}) ;{ }^{13} \mathrm{C} \mathrm{NMR}\left(101 \mathrm{MHz}, \mathrm{CDCl}_{3}\right) \delta$ 216.7, 215.3, 175.9, 171.3, 68.7, 61.0, 56.1, 49.8, 49.0, 47.7, 46.2, 42.8, 41.7, 39.2, 36.3, $33.9,32.9,31.8,31.7,31.6,30.7,26.5,25.9,25.3,21.2,20.6,20.2,18.8,18.1,17.7,17.5$, 15.6, -4.5, -4.7; IR (KBr): $v_{\max }=2957,2872,1723,1462,1367,1252,1091,837 \mathrm{~cm}^{-1}$; HRMS (ESI, $m / z$ ): calcd for $\mathrm{C}_{36} \mathrm{H}_{65} \mathrm{O}_{7} \mathrm{Si}[\mathrm{M}+\mathrm{H}]^{+}$637.4494; found 637.4498.

Compound 18: TLC (petroleum ether:EtOAc, $1: 1 \mathrm{v} / \mathrm{v}): R_{f}=0.3 ;[\alpha]_{\mathrm{D}}^{25}+9.4(c 1.66$, $\mathrm{CHCl}_{3}$ ); ${ }^{1} \mathrm{H}$ NMR $\left(400 \mathrm{MHz}, \mathrm{CDCl}_{3}\right) \delta 6.91$ (s, 1H), $4.25-4.09$ (m, 2H), 4.01 (quintet, $J$ $=5.6, \mathrm{~Hz}, 1 \mathrm{H}), 3.14(\mathrm{t}, J=9.4 \mathrm{~Hz}, 1 \mathrm{H}), 2.40(\mathrm{~d}, J=5.9 \mathrm{~Hz}, 2 \mathrm{H}), 2.03(\mathrm{~s}, 3 \mathrm{H}), 2.00-1.81$ (m, 2H), $1.81-1.53$ (m, 6H), $1.53-1.34(\mathrm{~m}, 4 \mathrm{H}), 1.34-1.18(\mathrm{~m}, 4 \mathrm{H}), 1.15(\mathrm{~s}, 3 \mathrm{H}), 1.04$ 
$-0.93(\mathrm{~m}, 2 \mathrm{H}), 0.98(\mathrm{~d}, J=6.8 \mathrm{~Hz}, 3 \mathrm{H}), 0.86(\mathrm{~d}, J=6.8 \mathrm{~Hz}, 3 \mathrm{H}), 0.84$ (s, 9H), 0.79 (d, $J$ $=6.8 \mathrm{~Hz}, 3 \mathrm{H}), 0.78(\mathrm{~d}, J=6.8 \mathrm{~Hz}, 3 \mathrm{H}), 0.75(\mathrm{~s}, 3 \mathrm{H}), 0.03(\mathrm{~s}, 3 \mathrm{H}), 0.01(\mathrm{~s}, 3 \mathrm{H}) ;{ }^{13} \mathrm{C} \mathrm{NMR}$ $\left(101 \mathrm{MHz}, \mathrm{CDCl}_{3}\right) \delta 207.9,206.6,176.3,171.3,165.0,144.1,68.8,61.3,51.8,50.2,48.0$, 43.1, 41.7, 39.1, 37.5, 35.0, 33.2, 32.0, 31.6, 31.4, 29.5, 28.1, 26.9, 25.8, 21.3, 20.6, 19.8, 19.4, 18.1, 17.7, 17.3, 15.6, -4.5, -4.8; IR (KBr): $v_{\max }=2958,2867,1741,1703,1462$, 1367, 1257, 1091, $836 \mathrm{~cm}^{-1}$; HRMS (ESI, $m / z$ ): calcd for $\mathrm{C}_{36} \mathrm{H}_{63} \mathrm{O}_{7} \mathrm{Si}[\mathrm{M}+\mathrm{H}]^{+}$635.4338; found 635.4338 .

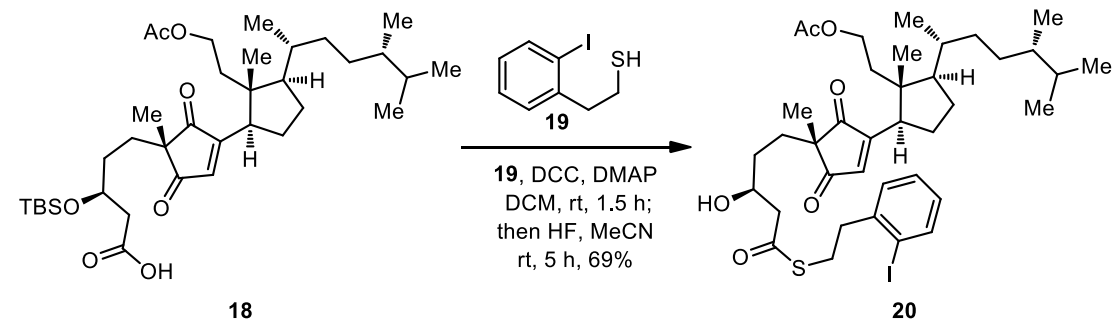

A mixture of 18 ( $76.0 \mathrm{mg}, 0.12 \mathrm{mmol}, 1.0$ equiv), DMAP ( $2.8 \mathrm{mg}, 0.024 \mathrm{mmol}, 0.2$ equiv) and DCC (34.8 mg, $0.17 \mathrm{mmol}, 1.4$ equiv) in DCM (4 mL) was stirred at room temperature for $5 \mathrm{~min}$ before thiol 19 ( $44.0 \mathrm{mg}, 0.17 \mathrm{mmol}, 1.4$ equiv) was added. After stirring at room temperature for $1.5 \mathrm{~h}, 4 \mathrm{~mL} \mathrm{MeCN}$ was added and $40 \%$ aq. $\mathrm{HF}(0.42$ ml $\times 4,8.4 \mathrm{mmol} \times 4,70$ equiv $\times 4$ ) was added in four portions every $1 \mathrm{~h}$. After completion of the addition of $40 \%$ aq. $\mathrm{HF}$ ( $4 \mathrm{~h}$ in total), the reaction mixture was stirred for another 1 h, quenched with aq. $\mathrm{NaHCO}_{3}$ and extracted with $\mathrm{DCM}(3 \times 10 \mathrm{~mL})$. The combined organic layers were washed with brine and dried over $\mathrm{Na}_{2} \mathrm{SO}_{4}$. Removal of the solvent under reduced pressure afforded the crude product, which was purified by flash chromatography ( $\mathrm{SiO}_{2}, 3: 1$ petroleum ether:EtOAc) to provide $20(63.2 \mathrm{mg}, 69 \%)$ as colorless oil.

Compound 20: TLC (petroleum ether:EtOAc, 2:1 v/v): $R_{f}=0.50$; $[\alpha]_{\mathrm{D}}^{25}+7.2(c 1.44$, $\left.\mathrm{CHCl}_{3}\right) ;{ }^{1} \mathrm{H} \mathrm{NMR}\left(400 \mathrm{MHz}, \mathrm{CDCl}_{3}\right) \delta 7.81(\mathrm{~d}, J=7.6 \mathrm{~Hz}, 1 \mathrm{H}), 7.34-7.20(\mathrm{~m}, 2 \mathrm{H})$, $6.96-6.86(\mathrm{~m}, 1 \mathrm{H}), 6.91(\mathrm{~s}, 1 \mathrm{H}), 4.26-4.12(\mathrm{~m}, 2 \mathrm{H}), 3.96-3.81(\mathrm{~m}, 1 \mathrm{H}), 3.21-3.07$ (m, 3H), $3.04-2.93(\mathrm{~m}, 2 \mathrm{H}), 2.73-2.52(\mathrm{~m}, 3 \mathrm{H}), 2.03(\mathrm{~s}, 3 \mathrm{H}), 2.00-1.80(\mathrm{~m}, 3 \mathrm{H}), 1.80$ 
$-1.69(\mathrm{~m}, 3 \mathrm{H}), 1.69-1.51(\mathrm{~m}, 3 \mathrm{H}), 1.51-1.34(\mathrm{~m}, 4 \mathrm{H}), 1.35-1.18(\mathrm{~m}, 3 \mathrm{H}), 1.16(\mathrm{~s}$, $3 \mathrm{H}), 1.05-0.91(\mathrm{~m}, 2 \mathrm{H}), 0.99(\mathrm{~d}, J=6.8 \mathrm{~Hz}, 3 \mathrm{H}), 0.86(\mathrm{~d}, J=6.8 \mathrm{~Hz}, 3 \mathrm{H}), 0.79(\mathrm{~d}, J=$ $6.8 \mathrm{~Hz}, 3 \mathrm{H}), 0.78(\mathrm{~d}, J=6.8 \mathrm{~Hz}, 3 \mathrm{H}), 0.75(\mathrm{~s}, 3 \mathrm{H}) ;{ }^{13} \mathrm{C} \mathrm{NMR}\left(101 \mathrm{MHz}, \mathrm{CDCl}_{3}\right) \delta 208.1$, 206.5, 199.1, 171.2, 164.9, 144.1, 142.4, 139.7, 130.2, 128.6, 128.6, 100.5, 68.4, 61.3, 51.7, 50.4, 50.3, 48.0, 43.0, 40.4, 39.1, 37.4, 35.0, 33.2, 31.6, 31.4, 31.3, 30.0, 29.0, 28.1, 26.9, 21.3, 20.6, 20.0, 19.4, 17.7, 17.4, 15.6; IR (KBr): $v_{\max }=2958,2871,1739,1698$, 1465, 1366, 1237, 1033, $750 \mathrm{~cm}^{-1}$; HRMS (DART, $\mathrm{m} / \mathrm{z}$ ): calcd for $\mathrm{C}_{38} \mathrm{H}_{59} \mathrm{INO}_{6} \mathrm{Si}$ $\left[\mathrm{M}+\mathrm{NH}_{4}\right]^{+}$784.3102; found 784.3103.

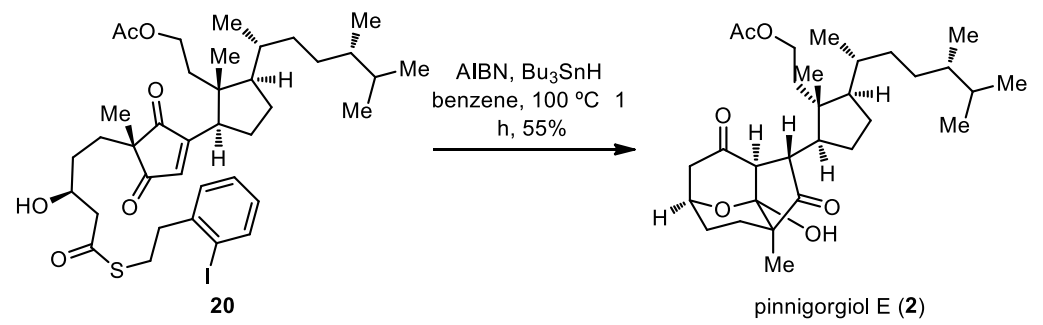

A solution of 20 ( $38.3 \mathrm{mg}, 0.05 \mathrm{mmol}, 1.0$ equiv), $\mathrm{AIBN}$ ( $8.2 \mathrm{mg}, 0.05 \mathrm{mmol}, 1.0$ equiv), and $\mathrm{Bu}_{3} \mathrm{SnH}(29.6 \mu \mathrm{L}, 0.11 \mathrm{mmol}, 2.2$ equiv) in benzene $(6.6 \mathrm{~mL})$ was degassed by freeze-pump-thaw for 3 times. The reaction mixture was heated to reflux at $100{ }^{\circ} \mathrm{C}$ for 1 $\mathrm{h}$ under argon. After the mixture was cooled to room temperature, the solvent was removed in vacuo. The crude was purified by flash chromatography $\left(\mathrm{SiO}_{2}, 50: 1 \rightarrow 2: 1\right.$ petroleum ether:EtOAc) to provide $2(14.0 \mathrm{mg}, 55 \%)$ as colorless oil.

Pinnigorgiol E (2): TLC (petroleum ether:Acetone, 3:1 v/v): $R_{f}=0.40 ;[\alpha]_{\mathrm{D}}^{25}-0.9(c 0.33$, $\left.\mathrm{CHCl}_{3}\right) ;{ }^{1} \mathrm{H} \mathrm{NMR}\left(400 \mathrm{MHz}, \mathrm{CDCl}_{3}\right) \delta 4.65(\mathrm{t}, J=6.0 \mathrm{~Hz}, 1 \mathrm{H}), 4.54(\mathrm{ddd}, J=11.6,11.6$, $4.0 \mathrm{~Hz}, 1 \mathrm{H}), 3.86$ (ddd, $J=11.6,11.6,6.0 \mathrm{~Hz}, 1 \mathrm{H}), 3.19$ (dd, $J=10.0,2.0 \mathrm{~Hz}, 1 \mathrm{H}), 3.06$ (s, 1H), 2.92 (dd, $J=16.4,7.2 \mathrm{~Hz}, 1 \mathrm{H}), 2.40$ (d, $J=16.4,1 \mathrm{H}), 2.13-2.02(\mathrm{~m}, 1 \mathrm{H}), 2.08$ - 1.97 (m, 2H), 2.06 (s, 3H), $1.96-1.88(\mathrm{~m}, 2 \mathrm{H}), 1.95-1.84(\mathrm{~m}, 1 \mathrm{H}), 1.69-1.58(\mathrm{~m}$, $1 \mathrm{H}), 1.61-1.51(\mathrm{~m}, 1 \mathrm{H}), 1.52-1.38(\mathrm{~m}, 1 \mathrm{H}), 1.50-1.39(\mathrm{~m}, 1 \mathrm{H}), 1.48-1.37(\mathrm{~m}, 1 \mathrm{H})$, $1.52-1.38(\mathrm{~m}, 1 \mathrm{H}), 1.46-1.38(\mathrm{~m}, 1 \mathrm{H}), 1.46-1.36(\mathrm{~m}, 1 \mathrm{H}), 1.45-1.35(\mathrm{~m}, 1 \mathrm{H}), 1.30$ $-1.18(\mathrm{~m}, 1 \mathrm{H}), 1.26-1.16(\mathrm{~m}, 1 \mathrm{H}), 1.12(\mathrm{~s}, 3 \mathrm{H}), 0.98(\mathrm{~d}, J=6.4 \mathrm{~Hz}, 3 \mathrm{H}), 0.99-0.91$ 
(m, 1H), $0.96-0.86(\mathrm{~m}, 1 \mathrm{H}), 0.90(\mathrm{~s}, 3 \mathrm{H}), 0.85(\mathrm{~d}, J=6.8 \mathrm{~Hz}, 3 \mathrm{H}), 0.78(\mathrm{~d}, J=6.8 \mathrm{~Hz}$, 3H), $0.77(\mathrm{~d}, J=6.8 \mathrm{~Hz}, 3 \mathrm{H}) ;{ }^{13} \mathrm{C} \mathrm{NMR}\left(101 \mathrm{MHz}, \mathrm{cdcl}_{3}\right) \delta 216.6,207.7,172.5,101.4$, 70.2, 61.3, 59.8, 49.8, 49.4, 48.1, 46.8, 45.9, 44.0, 39.0, 36.2, 33.3, 32.6, 31.9, 31.5, 27.2, $26.6,24.2,24.1,21.2,20.49,20.45,17.6,16.9,15.5,12.2$; IR $(\mathrm{KBr}): v_{\max }=3429,2959$, 2930, 2870, 1737, 1707, 1465, 1367, 1261, 1028, 801, $760 \mathrm{~cm}^{-1}$; HRMS (ESI, m/z): calcd for $\mathrm{C}_{30} \mathrm{H}_{49} \mathrm{O}_{6}[\mathrm{M}+\mathrm{H}]^{+}$505.3524; found 505.3520.

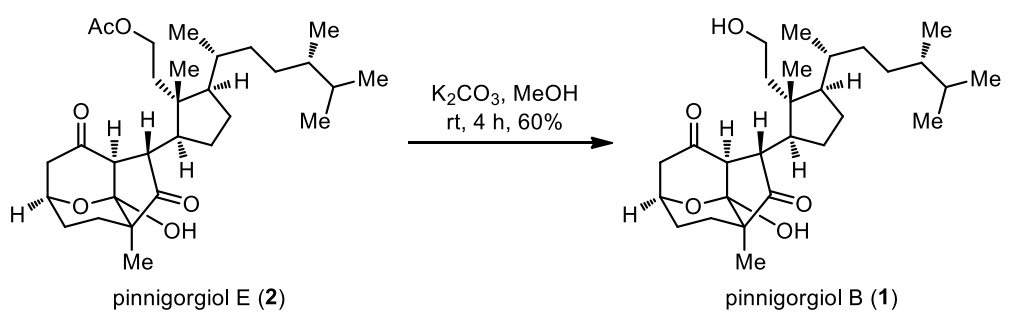

To a solution of 2 (13.0 mg, 0.026 mmol, 1.0 equiv) in $1 \mathrm{~mL} \mathrm{MeOH}$ was added $\mathrm{K}_{2} \mathrm{CO}_{3}$ (5.3 mg, $0.039 \mathrm{mmol}, 1.5$ equiv) at room temperature. After stirring at this temperature for $4 \mathrm{~h}$, the solvent was removed under reduced pressure to afford the crude product, which was purified by flash chromatography $\left(\mathrm{SiO}_{2}, \mathrm{EtOAc}\right)$ to provide $1(7.1 \mathrm{mg}, 60 \%)$ as colorless oil.

Pinnigorgiol B (1): TLC (EtOAc): $R_{f}=0.30 ;[\alpha]_{\mathrm{D}}^{23}+14.0\left(c 0.28, \mathrm{CHCl}_{3}\right) ;{ }^{1} \mathrm{H} \mathrm{NMR}(400$ $\left.\mathrm{MHz}, \mathrm{CDCl}_{3}\right) \delta 4.62(\mathrm{t}, J=6.0 \mathrm{~Hz}, 1 \mathrm{H}), 3.91-3.71(\mathrm{~m}, 2 \mathrm{H}), 3.21(\mathrm{~d}, J=10.8 \mathrm{~Hz}, 1 \mathrm{H})$, $3.20(\mathrm{br} \mathrm{s}, 1 \mathrm{H}), 2.91(\mathrm{dd}, J=16.4,6.8 \mathrm{~Hz}, 1 \mathrm{H}), 2.40(\mathrm{~d}, J=16.4 \mathrm{~Hz}, 1 \mathrm{H}), 2.25-2.12(\mathrm{~m}$, 1H), 2.00 (tt, $J=14.0,4.8 \mathrm{~Hz}, 1 \mathrm{H}), 1.89$ (ddd, $J=16.0,5.6 \mathrm{~Hz}, 5.2 \mathrm{~Hz}, 1 \mathrm{H}), 1.82-1.60$ (m, 4H), $1.60-1.48(\mathrm{~m}, 2 \mathrm{H}), 1.48-1.12(\mathrm{~m}, 8 \mathrm{H}), 1.11(\mathrm{~s}, 3 \mathrm{H}), 0.94-0.89(\mathrm{~m}, 2 \mathrm{H}), 0.90$ $(\mathrm{d}, J=6.8 \mathrm{~Hz}, 3 \mathrm{H}), 0.88(\mathrm{~s}, 3 \mathrm{H}), 0.85(\mathrm{~d}, J=6.8 \mathrm{~Hz}, 3 \mathrm{H}), 0.78(\mathrm{~d}, J=6.8 \mathrm{~Hz}, 3 \mathrm{H}), 0.77$ $(\mathrm{d}, J=6.8 \mathrm{~Hz}, 3 \mathrm{H}) .{ }^{13} \mathrm{C} \mathrm{NMR}\left(101 \mathrm{MHz}, \mathrm{CDCl}_{3}\right) \delta 216.5,208.0,101.3,70.3,59.9,59.2$, 50.0, 49.4, 47.51, 47.44, 46.6, 43.9, 39.6, 39.0, 33.3, 32.6, 31.8, 31.5, 26.6, 25.9, 24.2, 24.1, 20.53, 20.45, 17.6, 17.1, 15.4, 12.1. IR (KBr): $v_{\max }=3390,2959,2878,1741,1704$, 1462, 1376, 1261, 1027, 801, $703 \mathrm{~cm}^{-1}$; HRMS (ESI, m/z): calcd for $\mathrm{C}_{28} \mathrm{H}_{47} \mathrm{O}_{5}[\mathrm{M}+\mathrm{H}]^{+}$ 463.3418; found 463.3418 . 


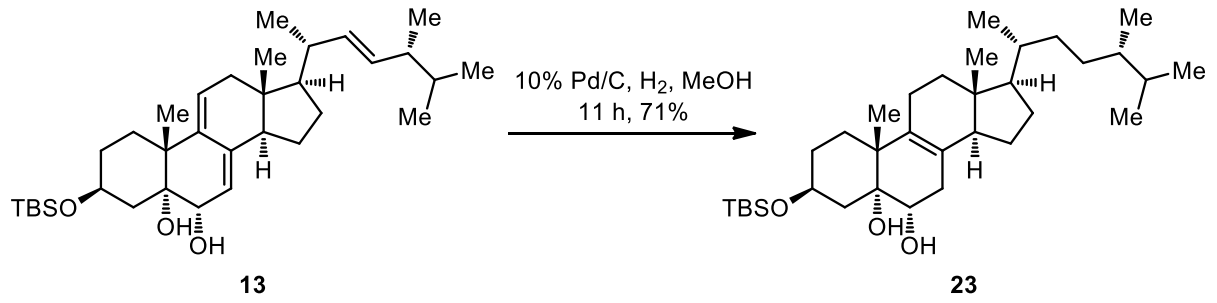

To a dried three-necked flask was sequentially added 10\% Pd/C (400.0 mg, $40 \mathrm{wt} \%), 91$ $\mathrm{mL} \mathrm{MeOH}$ and 13 (1.0 g, $1.83 \mathrm{mmol}, 1.0$ equiv) under argon at room temperature. The flask was purged with hydrogen and stirred under 1 atm $\mathrm{H}_{2}$. After $11 \mathrm{~h}$, the flask was purged with argon and the reaction was quenched with $1 \mathrm{~mL} \mathrm{H}_{2} \mathrm{O}$. Then the mixture was filtered through a short plug of Celite, and the filter cake was washed with EtOAc $(2 \times 30$ $\mathrm{mL}$ ). The filtrate was concentrated under vacuum. The crude residue was purified by flash chromatography ( $\mathrm{SiO}_{2}, 20: 1$ petroleum ether:EtOAc) to provide $\mathbf{2 3}$ (713.8 $\left.\mathrm{mg}, 71 \%\right)$ as a white solid.

Spectroscopic data of compound $\mathbf{2 3}$ was provided in Page S13.

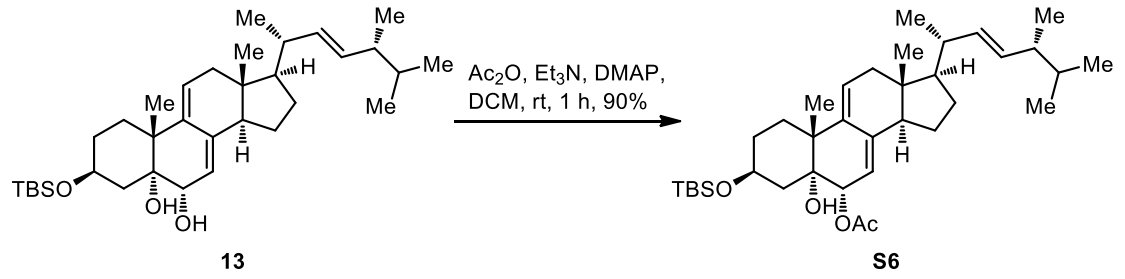

To a solution of 13 ( $3.0 \mathrm{~g}, 5.5 \mathrm{mmol}, 1.0$ equiv) in $50 \mathrm{~mL}$ dry DCM was added DMAP (72.0 mg, $0.59 \mathrm{mmol}, 0.1$ equiv), $\mathrm{Et}_{3} \mathrm{~N}$ ( $2.3 \mathrm{~mL}, 16.5 \mathrm{mmol}, 3.0$ equiv) and $\mathrm{Ac}_{2} \mathrm{O}$ (0.78 $\mathrm{mL}, 8.3 \mathrm{mmol}, 1.5$ equiv) at room temperature. After stirred at this temperature for $1 \mathrm{~h}$, the reaction mixture was quenched with $\mathrm{H}_{2} \mathrm{O}(50 \mathrm{~mL})$ and extracted with DCM $(3 \times 50$ $\mathrm{mL}$ ). The combined organic layers were washed with brine and dried over $\mathrm{Na}_{2} \mathrm{SO}_{4}$. Removal of the solvent under reduced pressure afforded the crude product, which was purified by flash chromatography $\left(\mathrm{SiO}_{2}, 12: 1\right.$ petroleum ether:EtOAc) to provide $\mathbf{S 6}$ (2.9 g, 90\%) as a white foam. 
Compound S6: TLC (petroleum ether:EtOAc, 8:1 v/v): $R_{f}=0.30 ;[\alpha]_{\mathrm{D}}^{25}+62.2(c 1.19$, $\left.\mathrm{CHCl}_{3}\right) ;{ }^{1} \mathrm{H} \mathrm{NMR}\left(400 \mathrm{MHz}, \mathrm{CDCl}_{3}\right) \delta 5.66(\mathrm{~d}, J=6.4 \mathrm{~Hz}, 1 \mathrm{H}), 5.34(\mathrm{~s}, 1 \mathrm{H}), 5.24(\mathrm{dd}, J$ $=15.2,7.2 \mathrm{~Hz}, 1 \mathrm{H}), 5.15(\mathrm{dd}, J=15.2,8.0 \mathrm{~Hz}, 1 \mathrm{H}), 5.00(\mathrm{~s}, 1 \mathrm{H}), 3.99(\mathrm{tt}, J=11.2,4.8$ $\mathrm{Hz}, 1 \mathrm{H}), 2.39-2.26(\mathrm{~m}, 2 \mathrm{H}), 2.16(\mathrm{~s}, 3 \mathrm{H}), 2.23-2.14(\mathrm{~m}, 1 \mathrm{H}), 2.03(\mathrm{q}, J=7.5 \mathrm{~Hz}, 1 \mathrm{H})$, $1.91-1.75(\mathrm{~m}, 6 \mathrm{H}), 1.67-1.60(\mathrm{~m}, 2 \mathrm{H}), 1.52-1.41(\mathrm{~m}, 3 \mathrm{H}), 1.41-1.28(\mathrm{~m}, 3 \mathrm{H}), 1.13$ $(\mathrm{s}, 3 \mathrm{H}), 1.01(\mathrm{~d}, J=6.8 \mathrm{~Hz}, 3 \mathrm{H}), 0.92(\mathrm{~d}, J=6.8 \mathrm{~Hz}, 3 \mathrm{H}), 0.88(\mathrm{~s}, 9 \mathrm{H}), 0.84(\mathrm{~d}, J=6.4$ $\mathrm{Hz}, 3 \mathrm{H}), 0.82(\mathrm{~d}, J=6.4 \mathrm{~Hz}, 3 \mathrm{H}), 0.54(\mathrm{~s}, 3 \mathrm{H}), 0.06$ (s, 3H), 0.05 (s, 3H); ${ }^{13} \mathrm{C}$ NMR $(101$ $\left.\mathrm{MHz}_{\mathrm{CDCl}}\right) \delta$ 171.0, 140.0, 139.2, 135.4, 132.4, 124.7, 116.5, 75.2, 74.1, 67.8, 56.1, 51.4, 42.9, 42.7, 42.3, 41.3, 40.5, 38.7, 33.2, 31.3, 30.1, 28.9, 26.1, 24.8, 23.1, 21.4, 20.8, 20.1, 19.8, 18.3, 17.7, 11.6, -4.3, -4.4; IR (KBr): $v_{\max }=2956,2870,1743,1471,1372$, 1235, 1105, $878 \mathrm{~cm}^{-1}$; HRMS (DART, $m / z$ ): calcd for $\mathrm{C}_{36} \mathrm{H}_{64} \mathrm{NO}_{4} \mathrm{Si}\left[\mathrm{M}+\mathrm{NH}_{4}\right]^{+} 602.4599$; found 602.4601 .

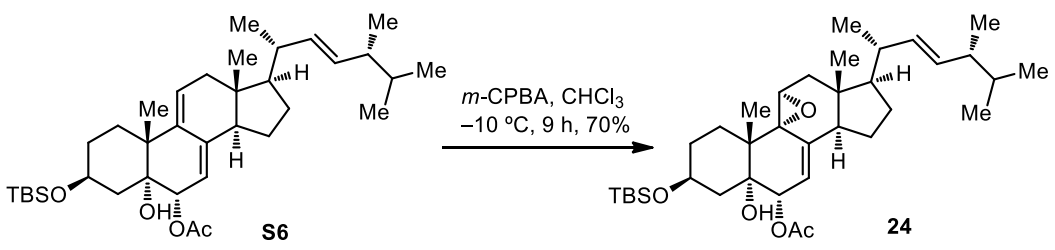

To a stirred solution of $\mathbf{S 6}\left(1.0 \mathrm{~g}, 1.7 \mathrm{mmol}, 1.0\right.$ equiv) in $20 \mathrm{~mL} \mathrm{CHCl}_{3}$ was added dropwise $85 \% \mathrm{~m}$-CPBA $\left(521.0 \mathrm{mg}, 2.6 \mathrm{mmol}, 1.5\right.$ equiv, as a solution in $\left.20 \mathrm{~mL} \mathrm{CHCl}_{3}\right)$ at $-10{ }^{\circ} \mathrm{C}$. After stirred at the same temperature for $9 \mathrm{~h}$, the reaction mixture was quenched with sat. aq. $\mathrm{Na}_{2} \mathrm{~S}_{2} \mathrm{O}_{3}$ and extracted with EtOAc $(3 \times 20 \mathrm{~mL})$. The combined organic layers were washed with brine, dried over $\mathrm{Na}_{2} \mathrm{SO}_{4}$ and concentrated in vacuo. The residue was purified by flash chromatography $\left(\mathrm{SiO}_{2}, 20: 1\right.$ petroleum ether:EtOAc) to furnish compound $24(720.0 \mathrm{mg}, 70 \%)$ as a white foam.

Compound 24: TLC (petroleum ether:EtOAc, 5:1 v/v): $R_{f}=0.60 ;[\alpha]_{\mathrm{D}}^{25}+34.6(c$ 1.24, $\left.\mathrm{CHCl}_{3}\right) ;{ }^{1} \mathrm{H} \mathrm{NMR}\left(400 \mathrm{MHz}, \mathrm{CDCl}_{3}\right) \delta 5.32(\mathrm{dt}, J=12.4,2.0 \mathrm{~Hz}, 2 \mathrm{H}), 5.22(\mathrm{dd}, J=15.6$, $7.6 \mathrm{~Hz}, 1 \mathrm{H}), 5.12(\mathrm{dd}, J=15.2,8.4 \mathrm{~Hz}, 1 \mathrm{H}), 3.97$ (tt, $J=10.4,4.8 \mathrm{~Hz}, 1 \mathrm{H}), 3.15$ (d, $J=$ $5.6 \mathrm{~Hz}, 1 \mathrm{H}), 2.38(\mathrm{t}, J=10.4 \mathrm{~Hz}, 1 \mathrm{H}), 2.17(\mathrm{~s}, 3 \mathrm{H}), 2.11(\mathrm{~d}, J=14.8,5.2 \mathrm{~Hz}, 1 \mathrm{H}), 2.00-$ 
$1.93(\mathrm{~m}, 3 \mathrm{H}), 1.92-1.81(\mathrm{~m}, 4 \mathrm{H}), 1.79-1.71(\mathrm{~m}, 2 \mathrm{H}), 1.57-1.51(\mathrm{~m}, 1 \mathrm{H}), 1.49-1.40$ (m, 4H), $1.33-1.25(\mathrm{~m}, 2 \mathrm{H}), 1.21(\mathrm{~s}, 3 \mathrm{H}), 0.98(\mathrm{~d}, J=6.8 \mathrm{~Hz}, 3 \mathrm{H}), 0.90(\mathrm{~d}, J=7.2 \mathrm{~Hz}$, 3H), 0.87 (s, 9H), 0.83 (d, $J=6.8 \mathrm{~Hz}, 3 \mathrm{H}), 0.81$ (d, $J=6.4 \mathrm{~Hz}, 3 \mathrm{H}), 0.58$ (s, 3H), 0.05 (s, $6 \mathrm{H}) ;{ }^{13} \mathrm{C}$ NMR $\left(101 \mathrm{MHz}, \mathrm{CDCl}_{3}\right) \delta 170.9,139.8,135.1,132.6,122.0,75.0,73.8,67.5$, $62.8,56.3,53.8,46.8,43.6,42.9,40.3,40.1,39.6,39.5,33.2,30.5,28.5,26.1,25.3,22.3$, $21.4,20.9,20.5,20.1,19.8,18.3,17.7,14.1,-4.3,-4.4 ; \mathrm{IR}(\mathrm{KBr}): v_{\max }=2956,2869$, 1742, 1461, 1371, 1235, 1108, $880 \mathrm{~cm}^{-1}$; HRMS (ESI, $\mathrm{m} / z$ ): calcd for $\mathrm{C}_{36} \mathrm{H}_{60} \mathrm{NaO}_{5} \mathrm{Si}$ $[\mathrm{M}+\mathrm{Na}]^{+}$623.4102; found 623.4101.

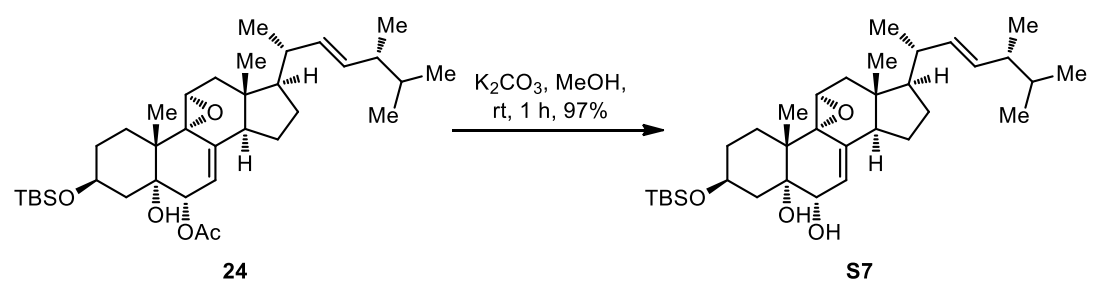

To a solution of 24 (990.0 mg, $1.7 \mathrm{mmol}, 1.0$ equiv) in $16 \mathrm{~mL} \mathrm{MeOH}$ was added $\mathrm{K}_{2} \mathrm{CO}_{3}$ (460.0 mg, $3.3 \mathrm{mmol}, 2.0$ equiv) at room temperature. After stirred at this temperature for $1 \mathrm{~h}$, the reaction mixture was quenched with $\mathrm{H}_{2} \mathrm{O}(15 \mathrm{~mL})$ and extracted with EtOAc $(3 \times 15 \mathrm{~mL})$. The combined organic layers were washed with brine and dried over $\mathrm{Na}_{2} \mathrm{SO}_{4}$. Removal of the solvent under reduced pressure afforded the crude product, which was purified by flash chromatography $\left(\mathrm{SiO}_{2}, 8: 1\right.$ petroleum ether:EtOAc) to provide $\mathbf{S 7}$ (910.0 $\mathrm{mg}, 97 \%)$ as a white foam.

Compound S7: TLC (petroleum ether:EtOAc, 5:1 v/v): $R_{f}=0.45 ;[\alpha]_{\mathrm{D}}^{25}+12.9(c 0.92$, $\left.\mathrm{CHCl}_{3}\right) ;{ }^{1} \mathrm{H} \mathrm{NMR}\left(400 \mathrm{MHz}, \mathrm{CDCl}_{3}\right) \delta 5.47(\mathrm{~s}, 1 \mathrm{H}), 5.22(\mathrm{dd}, J=15.6,8.0 \mathrm{~Hz}, 1 \mathrm{H}), 5.13$ (dd, $J=15.2,8.0 \mathrm{~Hz}, 1 \mathrm{H}), 4.06-3.86(\mathrm{~m}, 2 \mathrm{H}), 3.19(\mathrm{~d}, J=5.6 \mathrm{~Hz}, 1 \mathrm{H}), 2.43-2.29(\mathrm{~m}$, $2 \mathrm{H}), 2.23(\mathrm{~d}, J=13.2 \mathrm{~Hz}, 1 \mathrm{H}), 2.14(\mathrm{dd}, J=15.2,5.6 \mathrm{~Hz}, 1 \mathrm{H}), 2.01-1.93(\mathrm{~m}, 2 \mathrm{H}), 1.91$ $-1.82(\mathrm{~m}, 3 \mathrm{H}), 1.81-1.72(\mathrm{~m}, 2 \mathrm{H}), 1.64-1.59(\mathrm{~m}, 1 \mathrm{H}), 1.54-1.38(\mathrm{~m}, 5 \mathrm{H}), 1.34-$ $1.26(\mathrm{~m}, 2 \mathrm{H}), 1.18$ (s, 3H), 0.99 (d, $J=6.4 \mathrm{~Hz}, 3 \mathrm{H}), 0.91$ (d, $J=7.2 \mathrm{~Hz}, 3 \mathrm{H}), 0.88$ (s, 9H), $0.83(\mathrm{~d}, J=6.8 \mathrm{~Hz}, 3 \mathrm{H}), 0.81(\mathrm{~d}, J=6.8 \mathrm{~Hz}, 3 \mathrm{H}), 0.59$ (s, 3H), $0.06(\mathrm{~s}, 6 \mathrm{H}) ;{ }^{13} \mathrm{C} \mathrm{NMR}$ 
$\left(101 \mathrm{MHz}, \mathrm{CDCl}_{3}\right) \delta 136.8,135.1,132.6,127.4,76.4,70.5,67.6,62.9,56.3,53.9,46.7$, $43.3,42.9,40.3,40.1,39.2,38.7,33.2,30.5,28.6,26.0,25.4,22.4,20.9,20.3,20.1,19.8$, $18.3,17.7,14.0,-4.46,-4.49 ; \mathrm{IR}(\mathrm{KBr}): v_{\max }=3466,2954,2870,1461,1383,1247$, 1090, 1005, $877 \mathrm{~cm}^{-1}$; HRMS (ESI, $\mathrm{m} / z$ ): calcd for $\mathrm{C}_{34} \mathrm{H}_{59} \mathrm{O}_{4} \mathrm{Si}[\mathrm{M}+\mathrm{H}]^{+} 559.4177$; found 559.4178 .

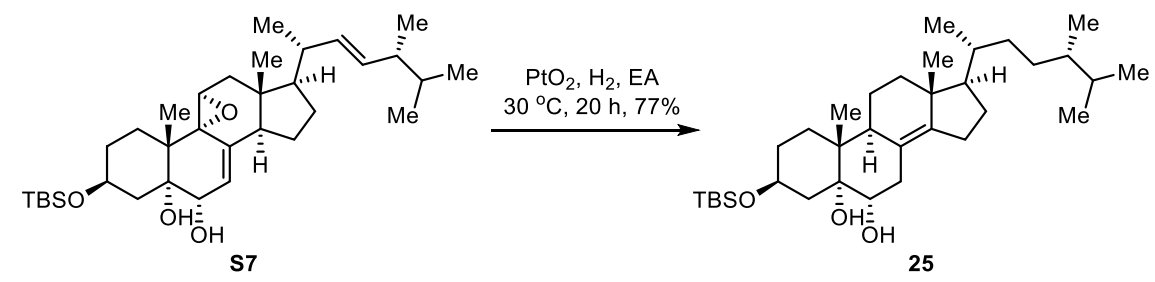

To a solution of $\mathbf{S} 7$ (40.0 mg, $0.072 \mathrm{mmol}, 1.0$ equiv) in $5 \mathrm{~mL}$ EtOAc was added $\mathrm{PtO}_{2}$ (20.0 mg, $50 \mathrm{wt} \%$ ) at room temperature [Note: $\mathrm{PtO}_{2}$ was purchased from Adamas (www.adamas-beta.com)]. The resulting mixture was stirred at $30{ }^{\circ} \mathrm{C}$ under $1 \mathrm{~atm} \mathrm{H}_{2}$ for $20 \mathrm{~h}$ before it was filtered through celite. Removal of the solvent under reduced pressure afforded the crude product, which was purified by flash chromatography $\left(\mathrm{SiO}_{2}, 16: 1\right.$ petroleum ether:EtOAc) to provide $\mathbf{2 5}(30.0 \mathrm{mg}, \mathbf{7 7 \%}$ ) as a white solid.

Compound 25: mp: $210.0-213{ }^{\circ} \mathrm{C}$; TLC (petroleum ether:EtOAc, 5:1 v/v): $R_{f}=0.50$; $[\alpha]_{\mathrm{D}}^{25}-9.7\left(\right.$ c 2.60, $\left.\mathrm{CHCl}_{3}\right) ;{ }^{1} \mathrm{H} \mathrm{NMR}\left(400 \mathrm{MHz}, \mathrm{CDCl}_{3}\right) \delta 4.01(\mathrm{tt}, J=11.2,4.8 \mathrm{~Hz}, 1 \mathrm{H})$, $3.58(\mathrm{dd}, J=11.2,5.6 \mathrm{~Hz}, 1 \mathrm{H}), 2.47$ (dd, $J=13.6,6.0 \mathrm{~Hz}, 1 \mathrm{H}), 2.38-2.29(\mathrm{~m}, 1 \mathrm{H}), 2.29$ $-2.13(\mathrm{~m}, 2 \mathrm{H}), 2.10-1.92(\mathrm{~m}, 3 \mathrm{H}), 1.90-1.71(\mathrm{~m}, 3 \mathrm{H}), 1.71-1.57(\mathrm{~m}, 3 \mathrm{H}), 1.56-$ $1.47(\mathrm{~m}, 3 \mathrm{H}), 1.46-1.31(\mathrm{~m}, 6 \mathrm{H}), 1.24-1.11(\mathrm{~m}, 3 \mathrm{H}), 1.07-0.95(\mathrm{~m}, 2 \mathrm{H}), 0.93(\mathrm{~d}, J=$ $6.4 \mathrm{~Hz}, 3 \mathrm{H}), 0.89$ (s, 9H), 0.85 (d, $J=6.8 \mathrm{~Hz}, 3 \mathrm{H}), 0.84$ (s, 3H), 0.83 (d, $J=8.0 \mathrm{~Hz}, 3 \mathrm{H})$, $0.78(\mathrm{~d}, J=6.8 \mathrm{~Hz}, 6 \mathrm{H}), 0.06(\mathrm{~s}, 6 \mathrm{H}) ;{ }^{13} \mathrm{C} \mathrm{NMR}\left(126 \mathrm{MHz}, \mathrm{CDCl}_{3}\right) \delta 144.3,123.4,76.7$, 71.0, 68.3, 56.6, 42.9, 40.2, 39.8, 39.5, 39.1, 37.2, 34.8, 34.0, 33.5, 31.5, 31.4, 31.0, 30.4, $27.0,25.9,25.8,20.5,19.8,19.2,18.2,18.1,17.6,16.6,15.4,-4.5,-4.6 ; \operatorname{IR}(\mathrm{KBr}): v_{\max }=$ 2954, 2868, 1470, 1384, 1253, 1081, 874, 835, $774 \mathrm{~cm}^{-1}$; HRMS (ESI, $\mathrm{m} / \mathrm{z}$ ): calcd for $\mathrm{C}_{34} \mathrm{H}_{62} \mathrm{NaO}_{3} \mathrm{Si}[\mathrm{M}+\mathrm{Na}]^{+}$569.4360; found 569.4362 . 


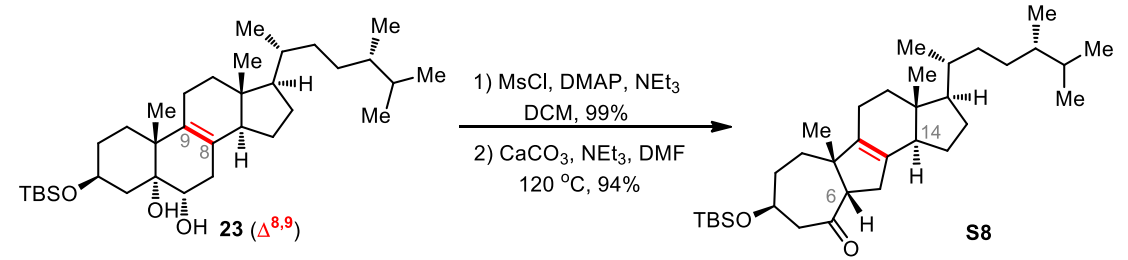

To a solution of 23 (1.00 g, $1.8 \mathrm{mmol}, 1.0$ equiv) and DMAP (22.3 mg, $0.18 \mathrm{mmol}, 0.1$ equiv) in $45 \mathrm{~mL}$ DCM was added $\mathrm{Et}_{3} \mathrm{~N}$ (1.02 mL, $7.3 \mathrm{mmol}, 4.0$ equiv) and $\mathrm{MsCl}$ (284 $\mu \mathrm{L}, 3.7 \mathrm{mmol}, 2.0$ equiv) at room temperature. After stirred at this temperature for $1 \mathrm{~h}$, the reaction mixture was quenched with saturated aq. $\mathrm{NaHCO}_{3}(10 \mathrm{~mL})$ and extracted with DCM $(3 \times 45 \mathrm{~mL})$. The combined organic layers were washed with brine, dried over $\mathrm{Na}_{2} \mathrm{SO}_{4}$ and concentrated in vacuo. The crude residue was purified by flash chromatography $\left(\mathrm{SiO}_{2}, 6: 1\right.$ petroleum ether:EtOAc) to provide the corresponding mesylate $(1.14 \mathrm{~g}, 99 \%)$ as a white foam.

To a solution of the mesylate $\left(1.94 \mathrm{~g}, 3.11 \mathrm{mmol}, 1.0\right.$ equiv) obtained above and $\mathrm{CaCO}_{3}$ (1.40 g, $14.0 \mathrm{mmol}, 4.5$ equiv) in $70 \mathrm{~mL}$ DMF was added $\mathrm{Et}_{3} \mathrm{~N}(2.6 \mathrm{~mL}, 18.7 \mathrm{mmol}, 6.0$ equiv) at room temperature. After stirred at $120{ }^{\circ} \mathrm{C}$ for $17 \mathrm{~h}$, the reaction mixture was quenched with saturated aq. $\mathrm{NaHCO}_{3}(30 \mathrm{~mL})$ and extracted with EtOAc $(4 \times 40 \mathrm{~mL})$. The combined organic layers were washed with brine, dried over $\mathrm{Na}_{2} \mathrm{SO}_{4}$ and concentrated in vacuo. The crude residue was purified by flash chromatography $\left(\mathrm{SiO}_{2}, 20: 1\right.$ petroleum ether:EtOAc) to provide $\mathbf{S 8}(1.54 \mathrm{~g}, 94 \%)$ as a white solid.

Compound S8: TLC (petroleum ether:EtOAc, 6:1 v/v): $R_{f}=0.45 ;[\alpha]_{\mathrm{D}}^{25}+0.20(c 0.91$, $\left.\mathrm{CHCl}_{3}\right) ;{ }^{1} \mathrm{H} \mathrm{NMR}\left(400 \mathrm{MHz}, \mathrm{CDCl}_{3}\right) \delta 3.81(\mathrm{tt}, J=8.8,4.4 \mathrm{~Hz}, 1 \mathrm{H}), 2.91(\mathrm{dd}, J=8.8$, $4.4 \mathrm{~Hz}, 1 \mathrm{H}), 2.74(\mathrm{t}, J=10.4 \mathrm{~Hz}, 1 \mathrm{H}), 2.68-2.58(\mathrm{~m}, 2 \mathrm{H}), 2.32-2.21(\mathrm{~m}, 1 \mathrm{H}), 2.18-$ $2.07(\mathrm{~m}, 1 \mathrm{H}), 2.07-2.01(\mathrm{~m}, 1 \mathrm{H}), 2.00-1.78(\mathrm{~m}, 3 \mathrm{H}), 1.78-1.62(\mathrm{~m}, 4 \mathrm{H}), 1.62-1.52$ (m, 1H), $1.47-1.30(\mathrm{~m}, 5 \mathrm{H}), 1.27(\mathrm{~s}, 3 \mathrm{H}), 1.24-1.13(\mathrm{~m}, 4 \mathrm{H}), 0.98-0.90(\mathrm{~m}, 2 \mathrm{H}), 0.94$ $(\mathrm{d}, J=6.8 \mathrm{~Hz}, 3 \mathrm{H}), 0.88(\mathrm{~s}, 9 \mathrm{H}), 0.85(\mathrm{~d}, J=6.8 \mathrm{~Hz}, 3 \mathrm{H}), 0.78(\mathrm{~d}, J=6.8 \mathrm{~Hz}, 3 \mathrm{H}), 0.77$ $(\mathrm{d}, J=6.8 \mathrm{~Hz}, 3 \mathrm{H}), 0.59(\mathrm{~s}, 3 \mathrm{H}), 0.07(\mathrm{~s}, 3 \mathrm{H}), 0.06(\mathrm{~s}, 3 \mathrm{H}) ;{ }^{13} \mathrm{C} \mathrm{NMR}\left(101 \mathrm{MHz}, \mathrm{CDCl}_{3}\right)$ $\delta 209.7,137.2,134.0,69.8,61.4,54.1,52.2,50.9,49.5,43.3,39.2,36.8,36.1,33.9,33.7$, 
33.0, 31.6, 30.9, 30.7, 29.1, 27.3, 26.0, 22.9, 20.7, 20.3, 19.1, 18.3, 17.7, 15.6, 11.2, -4.6, -4.7 ; IR (KBr): $v_{\max }=2955,2859,1697,1462,1376,1255,1084,837,735 \mathrm{~cm}^{-1}$; HRMS (DART, $m / z$ ): calcd for $\mathrm{C}_{34} \mathrm{H}_{61} \mathrm{O}_{2} \mathrm{Si}[\mathrm{M}+\mathrm{H}]^{+}$529.4435; found 529.4424 .

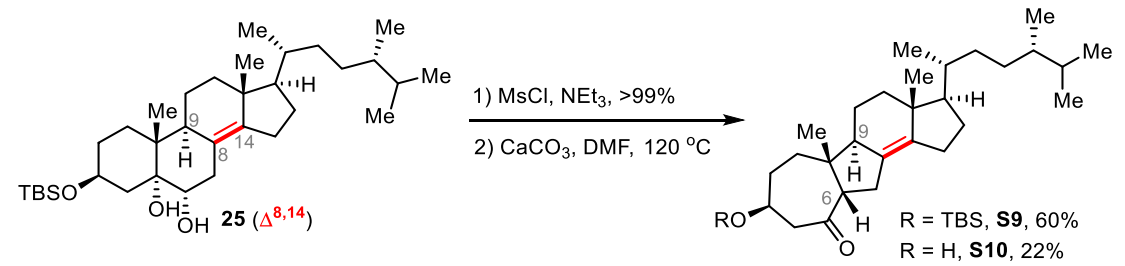

To a solution of 25 (67.4 mg, $0.12 \mathrm{mmol}, 1.0$ equiv) and DMAP (1.5 mg, $0.012 \mathrm{mmol}$, 0.1 equiv) in $3.7 \mathrm{~mL}$ DCM was added $\mathrm{Et}_{3} \mathrm{~N}$ ( $33.3 \mu \mathrm{L}, 0.24 \mathrm{mmol}, 2.0$ equiv) and $\mathrm{MsCl}$ (11.2 $\mu \mathrm{L}, 0.14 \mathrm{mmol}, 1.2$ equiv) at room temperature. After stirred at this temperature for $3 \mathrm{~h}$, the reaction mixture was quenched with saturated aq. $\mathrm{NaHCO}_{3}(2 \mathrm{~mL})$ and extracted with DCM $(3 \times 5 \mathrm{~mL})$. The combined organic layers were washed with brine, dried over $\mathrm{Na}_{2} \mathrm{SO}_{4}$ and concentrated in vacuo. The crude residue was purified by flash chromatography ( $\mathrm{SiO}_{2}, 8: 1$ petroleum ether:EtOAc) to provide the corresponding mesylate $(78.0 \mathrm{mg},>99 \%)$ as a white foam.

To a solution of the mesylate ( $78.0 \mathrm{mg}, 0.12 \mathrm{mmol}, 1.0$ equiv) obtained above in $3.9 \mathrm{~mL}$ DMF was added $\mathrm{CaCO}_{3}(55.3 \mathrm{mg}, 0.55 \mathrm{mmol}, 4.5$ equiv) at room temperature. After stirred at $120{ }^{\circ} \mathrm{C}$ for $7 \mathrm{~h}$, the reaction mixture was quenched with saturated aq. $\mathrm{NaHCO}_{3}$ $(30 \mathrm{~mL})$ and extracted with EtOAc $(4 \times 5 \mathrm{~mL})$. The combined organic layers were washed with brine, dried over $\mathrm{Na}_{2} \mathrm{SO}_{4}$ and concentrated in vacuo. The crude residue was purified by flash chromatography $\left(\mathrm{SiO}_{2}, 20: 1 \rightarrow 10: 1 \rightarrow 1: 1\right.$ petroleum ether:EtOAc) to provide $\mathbf{S 9}$ (38.4 $\mathrm{mg}, 60 \%)$ and $\mathbf{S 1 0}(11.0 \mathrm{mg}, 22 \%)$ as white solids.

Compound S9: TLC (petroleum ether:EtOAc, 10:1 v/v): $R_{f}=0.90 ;[\alpha]_{\mathrm{D}}^{25}-37.3(c 0.97$, $\left.\mathrm{CHCl}_{3}\right) ;{ }^{1} \mathrm{H} \mathrm{NMR}\left(400 \mathrm{MHz}, \mathrm{CDCl}_{3}\right) \delta 3.85-3.75(\mathrm{~m}, 1 \mathrm{H}), 3.03(\mathrm{dd}, J=8.4,3.2 \mathrm{~Hz}$, 1H), $2.72-2.55(\mathrm{~m}, 3 \mathrm{H}), 2.27-1.98(\mathrm{~m}, 3 \mathrm{H}), 1.96-1.83(\mathrm{~m}, 5 \mathrm{H}), 1.78-1.67(\mathrm{~m}, 1 \mathrm{H})$, $1.63-1.50(\mathrm{~m}, 1 \mathrm{H}), 1.48-1.26(\mathrm{~m}, 6 \mathrm{H}), 1.26-1.07(\mathrm{~m}, 4 \mathrm{H}), 1.04-0.94(\mathrm{~m}, 2 \mathrm{H}), 0.916$ 
(d, $J=6.8 \mathrm{~Hz}, 3 \mathrm{H}), 0.915$ (s, 3H), $0.88(\mathrm{~s}, 9 \mathrm{H}), 0.85$ (d, $J=6.8 \mathrm{~Hz}, 3 \mathrm{H}), 0.82(\mathrm{~s}, 3 \mathrm{H})$, $0.78(\mathrm{~d}, J=6.8 \mathrm{~Hz}, 3 \mathrm{H}), 0.77(\mathrm{~d}, J=6.8 \mathrm{~Hz}, 3 \mathrm{H}), 0.07$ (s, 3H), 0.05 (s, 3H); ${ }^{13} \mathrm{C}$ NMR $\left(101 \mathrm{MHz}, \mathrm{CDCl}_{3}\right) \delta 211.5,140.0,130.1,70.6,59.3,55.9,54.2,50.0,47.1,43.5,39.2$, 38.0, 35.6, 33.7, 33.6, 33.0, 31.7, 30.4, 27.8, 27.8, 25.9, 24.6, 22.9, 20.6, 19.6, 19.2, 19.1, 18.2, 17.8, 15.6, -4.6, -4.7; IR (KBr): $v_{\max }=2959,2858,1706,1461,1401,1257,1110$, 837, $778 \mathrm{~cm}^{-1}$; HRMS (ESI, m/z): calcd for $\mathrm{C}_{34} \mathrm{H}_{61} \mathrm{O}_{2} \mathrm{Si}[\mathrm{M}+\mathrm{H}]^{+}$529.4435; found 529.4437.

Compound S10: TLC (petroleum ether:EtOAc, 1:1 v/v): $R_{f}=0.50 ;[\alpha]_{\mathrm{D}}^{25}-30.2(c 0.96$, $\mathrm{CHCl}_{3}$ ); ${ }^{1} \mathrm{H} \mathrm{NMR}\left(400 \mathrm{MHz}, \mathrm{CDCl}_{3}\right) \delta 3.85$ (tt, $\left.J=9.6,4.8 \mathrm{~Hz}, 1 \mathrm{H}\right), 2.96(\mathrm{dd}, J=8.4$, $3.2 \mathrm{~Hz}, 1 \mathrm{H}), 2.80-2.73(\mathrm{~m}, 1 \mathrm{H}), 2.69(\mathrm{t}, J=10.1 \mathrm{~Hz}, 1 \mathrm{H}), 2.64-2.52(\mathrm{~m}, 1 \mathrm{H}), 2.25-$ $2.02(\mathrm{~m}, 3 \mathrm{H}), 2.00-1.73(\mathrm{~m}, 6 \mathrm{H}), 1.63-1.52(\mathrm{~m}, 2 \mathrm{H}), 1.48-1.27$ (m, 6H), $1.27-1.10$ (m, 3H), $1.08-0.95(\mathrm{~m}, 2 \mathrm{H}), 0.93(\mathrm{~s}, 3 \mathrm{H}), 0.92(\mathrm{~d}, J=6.8 \mathrm{~Hz}, 3 \mathrm{H}), 0.86(\mathrm{~d}, J=6.8 \mathrm{~Hz}$, $3 \mathrm{H}), 0.83(\mathrm{~s}, 3 \mathrm{H}), 0.78(\mathrm{~d}, J=6.8 \mathrm{~Hz}, 3 \mathrm{H}), 0.77(\mathrm{~d}, J=6.8 \mathrm{~Hz}, 3 \mathrm{H}) ;{ }^{13} \mathrm{C} \mathrm{NMR}(101 \mathrm{MHz}$, $\left.\mathrm{CDCl}_{3}\right) \delta 210.9,140.2,129.8,70.2,59.7,55.9,53.8,50.0,47.2,43.5,39.2,38.0,35.6$, 33.6, 33.6, 32.5, 31.7, 30.5, 28.1, 27.8, 24.6, 23.4, 20.6, 19.6, 19.2, 19.1, 17.8, 15.6; IR $(\mathrm{KBr}): v_{\max }=3186,2955,2867,1685,1465,1366,1266,1048,757 \mathrm{~cm}^{-1}$; HRMS (ESI, $m / z$ ): calcd for $\mathrm{C}_{28} \mathrm{H}_{46} \mathrm{NaO}_{2}[\mathrm{M}+\mathrm{Na}]^{+} 437.3390$; found 437.3389 .

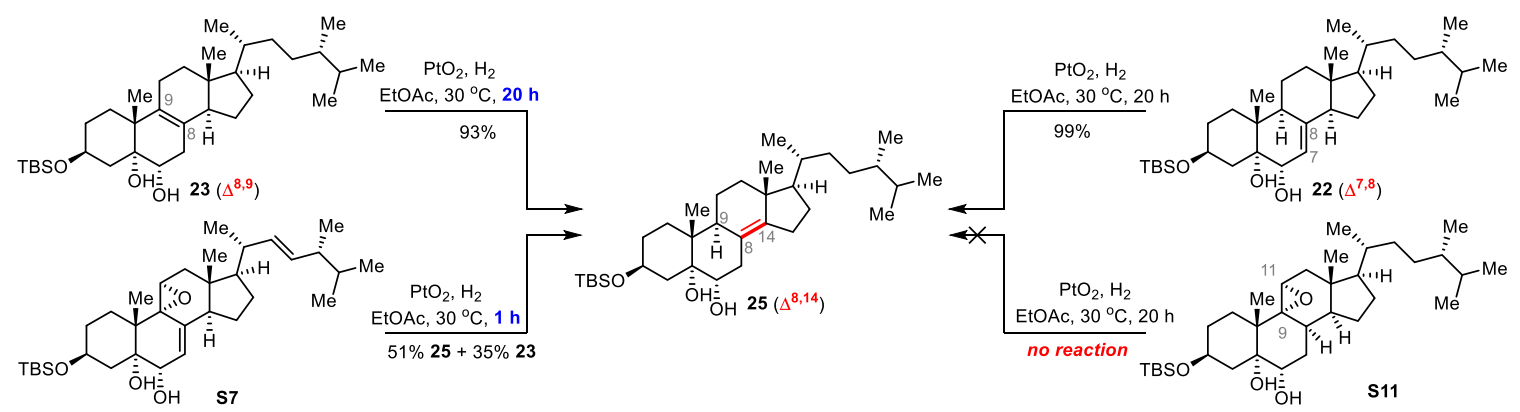

22 to 25: To a solution of $22(40.0 \mathrm{mg}, 0.073 \mathrm{mmol}, 1.0$ equiv) in $5 \mathrm{~mL}$ EtOAc was added $\mathrm{PtO}_{2}(20.0 \mathrm{mg}, 50 \mathrm{wt} \%)$ at room temperature [Note: $\mathrm{PtO}_{2}$ was purchased from Adamas (www.adamas-beta.com)]. The resulting mixture was stirred at $30{ }^{\circ} \mathrm{C}$ under $1 \mathrm{~atm} \mathrm{H}_{2}$ for $20 \mathrm{~h}$ before it was filtered through celite. Removal of the solvent under reduced pressure 
afforded the crude product, which was purified by flash chromatography $\left(\mathrm{SiO}_{2}, 16: 1\right.$ petroleum ether:EtOAc) to provide 25 (40.5 mg, 99\%) as a white solid.

23 to 25: To a solution of 23 (40.0 $\mathrm{mg}, 0.073 \mathrm{mmol}, 1.0$ equiv) in $5 \mathrm{~mL}$ EtOAc was added $\mathrm{PtO}_{2}(20.0 \mathrm{mg}, 50 \mathrm{wt} \%)$ at room temperature [Note: $\mathrm{PtO}_{2}$ was purchased from Adamas (www.adamas-beta.com)]. The resulting mixture was stirred at $30^{\circ} \mathrm{C}$ under 1 atm $\mathrm{H}_{2}$ for $20 \mathrm{~h}$ before it was filtered through celite. Removal of the solvent under reduced pressure afforded the crude product, which was purified by flash chromatography $\left(\mathrm{SiO}_{2}, 16: 1\right.$ petroleum ether:EtOAc) to provide $25(37.3 \mathrm{mg}, 93 \%)$ as a white solid.

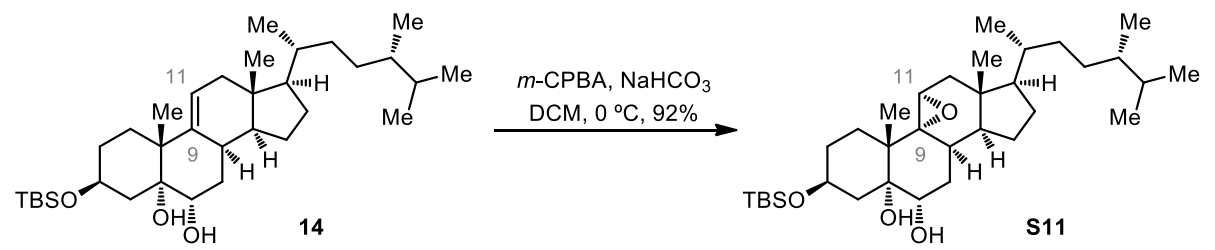

To a stirred solution of 14 (100.0 mg, $0.18 \mathrm{mmol}, 1.0$ equiv) in $3.7 \mathrm{~mL}$ DCM was added $\mathrm{NaHCO}_{3}(23.1 \mathrm{mg}, 0.28 \mathrm{mmol}, 1.5$ equiv) and 85\% m-CPBA (44.6 mg, $0.22 \mathrm{mmol}, 1.2$ equiv) at $0{ }^{\circ} \mathrm{C}$. After stirred at the same temperature for $0.5 \mathrm{~h}$, the reaction mixture was quenched with sat. aq. $\mathrm{NaHCO}_{3}$ and extracted with EtOAc $(3 \times 5 \mathrm{~mL})$. The combined organic layers were washed with brine, dried over $\mathrm{Na}_{2} \mathrm{SO}_{4}$ and concentrated in vacuo. The residue was purified by flash chromatography $\left(\mathrm{SiO}_{2}, 10: 1 \rightarrow 8: 1 \rightarrow 4: 1\right.$ petroleum ether:EtOAc) to furnish compound $\mathbf{S 1 1}(94.7 \mathrm{mg}, 92 \%)$ as a white solid.

Compound S11: mp: $251.7-253.2^{\circ} \mathrm{C}$; TLC (petroleum ether:EtOAc, 4:1 v/v): $R_{f}=0.42$; $[\alpha]_{\mathrm{D}}^{25}+32.1\left(c 0.39, \mathrm{CHCl}_{3}\right) ;{ }^{1} \mathrm{H} \mathrm{NMR}\left(400 \mathrm{MHz}, \mathrm{CDCl}_{3}\right) \delta 3.95(\mathrm{tt}, J=11.2,4.8 \mathrm{~Hz}, 1 \mathrm{H})$, $3.71(\mathrm{~d}, J=7.2 \mathrm{~Hz}, 1 \mathrm{H}), 2.90(\mathrm{~d}, J=6.4 \mathrm{~Hz}, 1 \mathrm{H}), 2.97-2.81(\mathrm{~m}, 1 \mathrm{H}), 2.47(\mathrm{~s}, 1 \mathrm{H}), 2.37$ (s, 1H), $2.25-2.10(\mathrm{~m}, 2 \mathrm{H}), 2.03(\mathrm{ddd}, J=13.2,4.8,2.0 \mathrm{~Hz}, 1 \mathrm{H}), 1.96-1.82(\mathrm{~m}, 2 \mathrm{H})$, $1.80-1.67(\mathrm{~m}, 3 \mathrm{H}), 1.63(\mathrm{~d}, J=14.8 \mathrm{~Hz}, 1 \mathrm{H}), 1.58-1.50(\mathrm{~m}, 1 \mathrm{H}), 1.50-1.32(\mathrm{~m}, 5 \mathrm{H})$, $1.32-1.16(\mathrm{~m}, 4 \mathrm{H}), 1.14(\mathrm{~s}, 3 \mathrm{H}), 1.11-1.04(\mathrm{~m}, 1 \mathrm{H}), 0.96-0.91(\mathrm{~m}, 2 \mathrm{H}), 0.87(\mathrm{~s}, 9 \mathrm{H})$, $0.87(\mathrm{~d}, J=6.4 \mathrm{~Hz}, 3 \mathrm{H}), 0.84(\mathrm{~d}, J=6.8 \mathrm{~Hz}, 3 \mathrm{H}), 0.77(\mathrm{~d}, J=6.8 \mathrm{~Hz}, 3 \mathrm{H}), 0.76(\mathrm{~d}, J=$ 
$6.8 \mathrm{~Hz}, 3 \mathrm{H}), 0.73(\mathrm{~s}, 3 \mathrm{H}), 0.05(\mathrm{~s}, 6 \mathrm{H}) ;{ }^{13} \mathrm{C} \mathrm{NMR}\left(101 \mathrm{MHz}, \mathrm{CDCl}_{3}\right) \delta$ 77.1, 69.7, 68.0, 66.0, 56.1, 54.3, 43.2, 42.7, 40.83, 40.80, 39.4, 39.2, 36.0, 33.5, 32.4, 31.6, 31.3, 30.7, $30.5,27.9,26.9,26.1,22.5,21.8,20.6,18.8,18.3,17.8,15.6,15.1,-4.4,-4.5 ;$ IR (KBr): $v_{\max }=3379,2954,2928,2855,1469,1367,1254,1171,1074,915,877 \mathrm{~cm}^{-1}$; HRMS (ESI, $m / z$ ): calcd for $\mathrm{C}_{34} \mathrm{H}_{62} \mathrm{NaO}_{4} \mathrm{Si}[\mathrm{M}+\mathrm{Na}]^{+}$585.4310; found 585.4312.

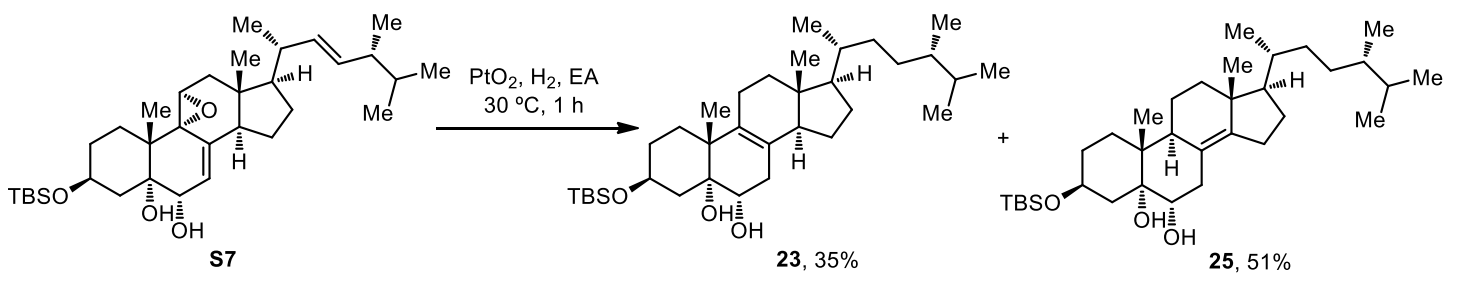

To a solution of $\mathbf{S 7}$ (30.0 mg, $0.054 \mathrm{mmol}, 1.0$ equiv) in $3.8 \mathrm{~mL}$ EtOAc was added $\mathrm{PtO}_{2}$ (15.0 mg, $50 \mathrm{wt} \%)$ at room temperature [Note: PtO $\mathrm{O}_{2}$ was purchased from Adamas (www.adamas-beta.com)]. The resulting mixture was stirred at $30^{\circ} \mathrm{C}$ under 1 atm $\mathrm{H}_{2}$ for $1 \mathrm{~h}$ before it was filtered through celite. Removal of the solvent under reduced pressure afforded the crude product, which was purified by flash chromatography $\left(\mathrm{SiO}_{2}, 20: 1\right.$ petroleum ether:EtOAc) to provide $23(10.1 \mathrm{mg}, 35 \%)$ and $25(15.1 \mathrm{mg}, 51 \%)$ as white solids.

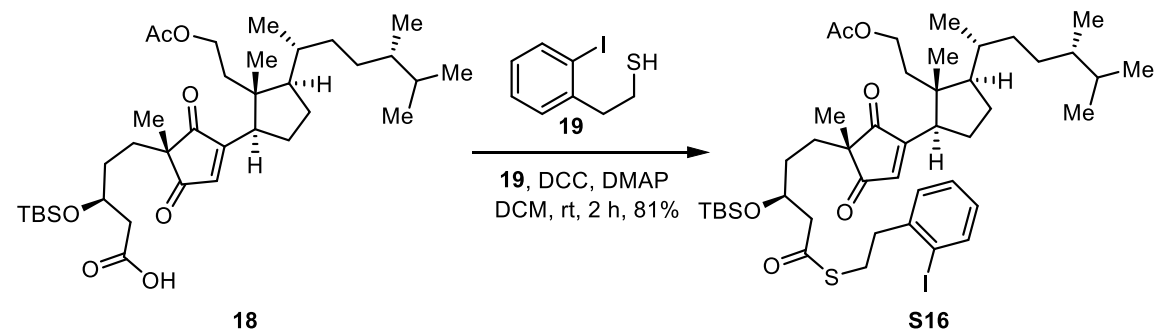

A mixture of 18 (49.5 mg, $0.08 \mathrm{mmol}, 1.0$ equiv), DMAP (1.9 mg, $0.016 \mathrm{mmol}, 0.2$ equiv) and DCC (22.5 mg, $0.11 \mathrm{mmol}, 1.4$ equiv) in DCM (2.3 mL) was stirred at room temperature for $5 \mathrm{~min}$ before thiol $19(28.7 \mathrm{mg}, 0.11 \mathrm{mmol}, 1.4$ equiv) was added. After stirring at room temperature for $2 \mathrm{~h}$, removal of the solvent under reduced pressure 
afforded the crude product, which was purified by flash chromatography $\left(\mathrm{SiO}_{2}, 10: 1\right.$ petroleum ether:EtOAc) to provide $\mathbf{S 1 6}$ (55.8 $\mathrm{mg}, 81 \%$ ) as colorless oil.

Compound S16: TLC (petroleum ether:EtOAc, 5:1 v/v): $R_{f}=0.70 ;[\alpha]_{\mathrm{D}}^{25}+19.7(c$ 2.08, $\left.\mathrm{CHCl}_{3}\right) ;{ }^{1} \mathrm{H} \mathrm{NMR}\left(400 \mathrm{MHz}, \mathrm{CDCl}_{3}\right) \delta 7.82(\mathrm{~d}, J=7.6 \mathrm{~Hz}, 1 \mathrm{H}), 7.34-7.25(\mathrm{~m}, 2 \mathrm{H})$, $6.98-6.85(\mathrm{~m}, 2 \mathrm{H}), 4.26-4.11(\mathrm{~m}, 2 \mathrm{H}), 4.08$ (quintet, $J=6.0 \mathrm{~Hz}, 1 \mathrm{H}), 3.19-3.11(\mathrm{~m}$, 1H), $3.11-3.02(\mathrm{~m}, 2 \mathrm{H}), 3.00-2.91(\mathrm{~m}, 2 \mathrm{H}), 2.68(\mathrm{dd}, J=14.4,7.2 \mathrm{~Hz}, 1 \mathrm{H}), 2.54(\mathrm{dd}, J$ $=14.4,4.8 \mathrm{~Hz}, 1 \mathrm{H}), 2.04(\mathrm{~s}, 3 \mathrm{H}), 1.99-1.82(\mathrm{~m}, 2 \mathrm{H}), 1.81-1.64(\mathrm{~m}, 5 \mathrm{H}), 1.64-1.55$ (m, 2H), $1.54-1.35(\mathrm{~m}, 4 \mathrm{H}), 1.31-1.18(\mathrm{~m}, 3 \mathrm{H}), 1.16(\mathrm{~s}, 3 \mathrm{H}), 1.07-0.91(\mathrm{~m}, 2 \mathrm{H}), 1.01$ (d, $J=6.8 \mathrm{~Hz}, 3 \mathrm{H}), 0.87(\mathrm{~d}, J=6.8 \mathrm{~Hz}, 3 \mathrm{H}), 0.85(\mathrm{~s}, 9 \mathrm{H}), 0.80(\mathrm{~d}, J=6.8 \mathrm{~Hz}, 3 \mathrm{H}), 0.79$ (d, $J=6.8 \mathrm{~Hz}, 3 \mathrm{H}), 0.77$ (s, 3H), 0.02 (s, 3H), $0.00(\mathrm{~s}, 3 \mathrm{H}) ;{ }^{13} \mathrm{C} \mathrm{NMR}\left(101 \mathrm{MHz}, \mathrm{CDCl}_{3}\right)$ $\delta$ 208.0, 206.6, 197.1, 171.2, 164.9, 144.0, 142.7, 139.7, 130.1, 128.6, 128.5, 100.4, 69.0, 61.3, 51.9, 51.3, 50.2, 48.0, 43.2, 40.6, 39.2, 37.5, 35.0, 33.3, 32.1, 31.6, 31.4, 29.5, 29.1, $28.2,26.9,25.9,21.2,20.6,19.7,19.5,18.1,17.8,17.3,15.6,-4.5,-4.7 ; \mathrm{IR}(\mathrm{KBr}): v_{\max }=$ 2957, 2871, 1741, 1699, 1464, 1366, 1256, 1091, $837 \mathrm{~cm}^{-1}$; HRMS (DART, $\mathrm{m} / \mathrm{z}$ ): calcd for $\mathrm{C}_{44} \mathrm{H}_{70} \mathrm{IO}_{6} \mathrm{SSi}[\mathrm{M}+\mathrm{H}]^{+}$881.3702; found 881.3699.

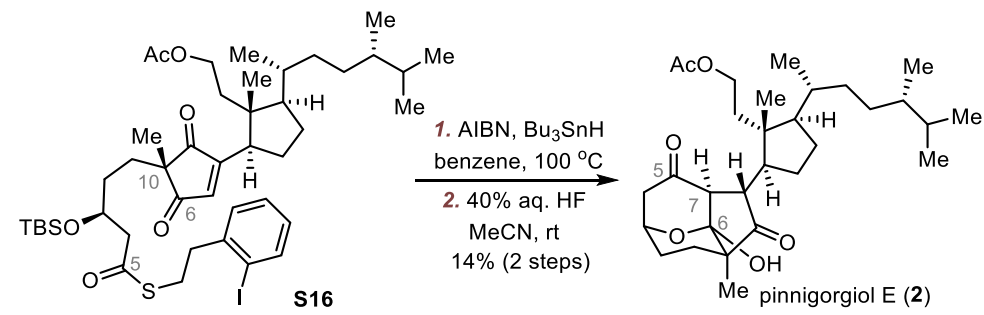

A solution of $\mathbf{S 1 6}(8.6 \mathrm{mg}, 0.0098 \mathrm{mmol}, 1.0$ equiv), AIBN (1.6 mg, $0.0098 \mathrm{mmol}, 1.0$ equiv), and $\mathrm{Bu}_{3} \mathrm{SnH}(5.8 \mu \mathrm{L}, 0.022 \mathrm{mmol}, 2.2$ equiv) in benzene $(1.3 \mathrm{~mL})$ was degassed by freeze-pump-thaw for 3 times. The reaction mixture was heated to reflux at $100{ }^{\circ} \mathrm{C}$ for $1 \mathrm{~h}$ under argon. After the mixture was cooled to room temperature, the solvent was removed in vacuo. To the crude mixture in $0.2 \mathrm{~mL} \mathrm{MeCN}$ was added $40 \%$ aq. $\mathrm{HF}(2.1 \mu \mathrm{L}$, $0.042 \mathrm{mmol}, 4.3$ equiv). After stirred at room temperature for $1 \mathrm{~h}$, the reaction mixture was quenched with aq. $\mathrm{NaHCO}_{3}$ and extracted with EtOAc $(3 \times 2 \mathrm{~mL})$. The combined 
organic layers were washed with brine and dried over $\mathrm{Na}_{2} \mathrm{SO}_{4}$. Removal of the solvent under reduced pressure afforded the crude product, which was purified by flash chromatography $\left(\mathrm{SiO}_{2}, 5: 1 \rightarrow 2: 1\right.$ petroleum ether:EtOAc) to provide $2(0.7 \mathrm{mg}, 14 \%)$ as a colorless oil. 


\section{NMR data comparison of synthetic and natural pinnigorgiols $B$ and $E$}

${ }^{1} \mathrm{H}$ NMR data comparison of synthetic and natural pinnigorgiol $\mathrm{E}$

\begin{tabular}{|c|c|c|c|}
\hline Position & $\begin{array}{c}\text { Natural pinnigorgiol } \mathrm{E}^{a} \\
\left(400 \mathrm{MHz} \text { in } \mathrm{CDCl}_{3}\right)\end{array}$ & $\begin{array}{c}\text { Synthetic pinnigorgiol E } \\
\left(400 \mathrm{MHz} \text { in } \mathrm{CDCl}_{3}\right)\end{array}$ & $\Delta \delta(\mathrm{ppm})$ \\
\hline \multirow[t]{2}{*}{1} & $1.38(\mathrm{~m})$ & $1.46-1.36(\mathrm{~m})$ & 0.03 \\
\hline & $1.25(\mathrm{~m})$ & $1.30-1.18(\mathrm{~m})$ & -0.01 \\
\hline \multirow[t]{2}{*}{2} & $2.03(\mathrm{~m})$ & $2.08-1.97(\mathrm{~m})$ & -0.01 \\
\hline & $1.48(\mathrm{~m})$ & $1.52-1.38(\mathrm{~m})$ & -0.03 \\
\hline 3 & $4.65(\mathrm{dd}, 6.8,4.8)$ & $4.65(t, 6.0)$ & 0 \\
\hline \multirow[t]{2}{*}{4} & $2.91(\mathrm{dd}, 16.4,7.2)$ & $2.92(\mathrm{dd}, 16.4,7.2)$ & 0.01 \\
\hline & $2.39(\mathrm{~d}, 16.4)$ & $2.40(\mathrm{~d}, 16.4)$ & 0.01 \\
\hline 7 & 3.05 (br s) & 3.06 (br s) & 0.01 \\
\hline 8 & $3.19(\mathrm{dd}, 10.0,2.0)$ & $3.19(\mathrm{dd}, 10.0,2.0)$ & 0 \\
\hline \multirow[t]{2}{*}{11} & $4.52(\mathrm{ddd}, 11.6,11.6,6.0)$ & $4.54(\mathrm{ddd}, 11.6,11.6,4.0)$ & 0.02 \\
\hline & $3.87(\mathrm{ddd}, 11.6,11.6,6.0)$ & $3.86(\mathrm{ddd}, 11.6,11.6,6.0)$ & -0.01 \\
\hline \multirow[t]{2}{*}{12} & $1.89(\mathrm{~m})$ & $1.95-1.84(\mathrm{~m})$ & -0.01 \\
\hline & $1.61(\mathrm{~m})$ & $1.69-1.58(\mathrm{~m})$ & 0.03 \\
\hline 14 & $2.04(\mathrm{~m})$ & $2.02-2.13(\mathrm{~m})$ & 0.03 \\
\hline 15 & $1.90(\mathrm{~m})$ & $1.96-1.88(\mathrm{~m})$ & 0.02 \\
\hline \multirow[t]{2}{*}{16} & $2.02(\mathrm{~m})$ & $2.08-1.97(\mathrm{~m})$ & 0.01 \\
\hline & $1.42(\mathrm{~m})$ & $1.52-1.38(\mathrm{~m})$ & 0.03 \\
\hline 17 & $1.42(\mathrm{~m})$ & $1.46-1.38(\mathrm{~m})$ & 0 \\
\hline 18 & $0.90(\mathrm{~s})$ & $0.90(\mathrm{~s})$ & 0 \\
\hline 19 & $1.12(\mathrm{~s})$ & $1.12(\mathrm{~s})$ & 0 \\
\hline 20 & $1.44(\mathrm{~m})$ & $1.48-1.37(\mathrm{~m})$ & -0.02 \\
\hline 21 & $0.98(\mathrm{~d}, 6.4)$ & $0.98(\mathrm{~d}, 6.4)$ & 0 \\
\hline \multirow[t]{2}{*}{22} & $1.45(\mathrm{~m})$ & $1.50-1.39(\mathrm{~m})$ & -0.01 \\
\hline & $0.90(\mathrm{~m})$ & $0.96-0.86(\mathrm{~m})$ & 0.01 \\
\hline \multirow[t]{2}{*}{23} & $1.37(\mathrm{~m})$ & $1.45-1.35(\mathrm{~m})$ & 0.03 \\
\hline & $0.90(\mathrm{~m})$ & $0.99-0.91(\mathrm{~m})$ & 0.05 \\
\hline 24 & $1.21(\mathrm{~m})$ & $1.26-1.16(\mathrm{~m})$ & 0 \\
\hline 25 & $1.56(\mathrm{~m})$ & $1.61-1.51(\mathrm{~m})$ & 0 \\
\hline 26 & $0.78(\mathrm{~d}, 6.8)$ & $0.78(\mathrm{~d}, 6.8)$ & 0 \\
\hline 27 & $0.85(\mathrm{~d}, 6.8)$ & $0.85(\mathrm{~d}, 6.8)$ & 0 \\
\hline 28 & $0.77(\mathrm{~d}, 6.8)$ & $0.78(\mathrm{~d}, 6.8)$ & 0 \\
\hline 11-OAc & $2.05(\mathrm{~s})$ & $2.06(\mathrm{~s})$ & 0.01 \\
\hline
\end{tabular}

${ }^{a}$ Chang, Y.-C.; Hwang, T.-L.; Sheu, J.-H.; Wu, Y.-C.; Sung, P.-J. Mar. Drugs 2016, 14, 218; Chang, Y.-C. Ph.D. Thesis, National Sun Yat-sen University, 2016. 


\section{${ }^{13} \mathrm{C}$ NMR data comparison of synthetic and natural pinnigorgiol $\mathrm{E}$}

\begin{tabular}{|c|c|c|c|}
\hline Position & $\begin{array}{c}\text { Natural pinnigorgiol } \mathrm{E}^{a} \\
\left(101 \mathrm{MHz} \text { in } \mathrm{CDCl}_{3}\right)\end{array}$ & $\begin{array}{c}\text { Synthetic pinnigorgiol E } \\
\left(101 \mathrm{MHz}^{-} \mathrm{CDCl}_{3}\right)\end{array}$ & $\Delta \delta(\mathrm{ppm})$ \\
\hline 1 & 26.6 & 26.6 & 0 \\
\hline 2 & 24.1 & 24.1 & 0 \\
\hline 3 & 70.2 & 70.2 & 0 \\
\hline 4 & 44.0 & 44.0 & 0 \\
\hline 5 & 101.4 & 101.4 & 0 \\
\hline 6 & 207.7 & 207.7 & 0 \\
\hline 7 & 59.9 & 59.8 & -0.1 \\
\hline 8 & 48.0 & 48.1 & 0.1 \\
\hline 9 & 216.6 & 216.6 & 0 \\
\hline 10 & 49.5 & 49.4 & -0.1 \\
\hline 11 & 61.3 & 61.3 & 0 \\
\hline 12 & 36.1 & 36.2 & 0.1 \\
\hline 13 & 46.8 & 46.8 & 0 \\
\hline 14 & 46.1 & 45.9 & -0.2 \\
\hline 15 & 27.0 & 27.2 & 0.2 \\
\hline 16 & 24.2 & 24.2 & 0 \\
\hline 17 & 49.8 & 49.8 & 0 \\
\hline 18 & 16.8 & 16.9 & 0.1 \\
\hline 19 & 12.1 & 12.2 & 0.1 \\
\hline 20 & 33.3 & 33.3 & 0 \\
\hline 21 & 20.4 & 20.45 & 0.05 \\
\hline 22 & 32.6 & 32.6 & 0 \\
\hline 23 & 31.8 & 31.9 & 0.1 \\
\hline 24 & 39.0 & 39.0 & 0 \\
\hline 25 & 31.5 & 31.5 & 0 \\
\hline 26 & 17.6 & 17.6 & 0 \\
\hline 27 & 20.4 & 20.49 & 0.09 \\
\hline 28 & 15.5 & 15.5 & 0 \\
\hline \multirow[t]{2}{*}{ 11-OAc } & 172.4 & 172.5 & 0.1 \\
\hline & 21.2 & 21.2 & 0 \\
\hline
\end{tabular}

${ }^{a}$ Chang, Y.-C.; Hwang, T.-L.; Sheu, J.-H.; Wu, Y.-C.; Sung, P.-J. Mar. Drugs 2016, 14, 218; Chang, Y.-C. Ph.D. Thesis, National Sun Yat-sen University, 2016. 


\section{${ }^{1} \mathrm{H}$ NMR data comparison of synthetic and natural pinnigorgiol $B$}

\begin{tabular}{|c|c|c|c|}
\hline Position & $\begin{array}{c}\text { Natural pinnigorgiol } \mathrm{B}^{\mathrm{a}} \\
\left(400 \mathrm{MHz} \text { in } \mathrm{CDCl}_{3}\right)\end{array}$ & $\begin{array}{l}\text { Synthetic pinnigorgiol B } \\
\left(400 \mathrm{MHz} \text { in } \mathrm{CDCl}_{3}\right)\end{array}$ & $\Delta \delta(\mathrm{ppm})$ \\
\hline \multirow[t]{2}{*}{1} & $1.42(\mathrm{~m})$ & $1.46-1.37(\mathrm{~m})$ & 0 \\
\hline & $1.26(\mathrm{~m})$ & $1.23-1.29(\mathrm{~m})$ & 0 \\
\hline \multirow[t]{2}{*}{2} & $1.43(\mathrm{~m})$ & $1.48-1.42(\mathrm{~m})$ & 0.02 \\
\hline & $2.01(\mathrm{tt}, 14.0,4.8)$ & $2.00(\mathrm{tt}, 14.0,4.8)$ & -0.01 \\
\hline 3 & $4.62(\mathrm{dd}, 6.8,4.8)$ & $4.62(\mathrm{t}, 6.0)$ & 0 \\
\hline \multirow[t]{2}{*}{4} & $2.91(\mathrm{dd}, 16.4,6.8)$ & $2.91(\mathrm{dd}, 16.4,6.8)$ & 0 \\
\hline & $2.40(\mathrm{~d}, 16.4)$ & $2.40(\mathrm{~d}, 16.4)$ & 0 \\
\hline 7 & 3.18 (br s) & 3.20 (br s) & 0.02 \\
\hline 8 & $3.22(\mathrm{dd}, 8.4,2.0)$ & $3.21(\mathrm{~d}, 10.8)$ & -0.01 \\
\hline 11 & $3.81(\mathrm{~m})$ & $3.91-3.71(\mathrm{~m})$ & 0 \\
\hline \multirow[t]{2}{*}{12} & $1.89(\mathrm{ddd}, 16.0,6.0,5.2)$ & $1.89(\mathrm{ddd}, 16.0,5.6,5.2)$ & 0 \\
\hline & $1.69(\mathrm{~m})$ & $1.76-1.68(\mathrm{~m})$ & 0.03 \\
\hline 14 & $2.19(\mathrm{~m})$ & $2.25-2.12(\mathrm{~m})$ & 0 \\
\hline 15 & $1.76(\mathrm{~m})$ & $1.82-1.73(\mathrm{~m})$ & 0.02 \\
\hline \multirow[t]{2}{*}{16} & $1.68(\mathrm{~m})$ & $-b$ & \\
\hline & $1.43(\mathrm{~m})$ & $1.48-1.42(\mathrm{~m})$ & 0.02 \\
\hline 17 & $1.53(\mathrm{~m})$ & $1.54-1.49(\mathrm{~m})$ & -0.01 \\
\hline 18 & $0.88(\mathrm{~s})$ & $0.88(\mathrm{~s})$ & 0 \\
\hline 19 & $1.11(\mathrm{~s})$ & $1.11(\mathrm{~s})$ & 0 \\
\hline 20 & $1.33(\mathrm{~m})$ & $1.37-1.31(\mathrm{~m})$ & 0.01 \\
\hline 21 & $0.90(\mathrm{~d}, 6.4)$ & $0.90(\mathrm{~d}, 6.8)$ & 0 \\
\hline \multirow[t]{2}{*}{22} & $1.44(\mathrm{~m})$ & $-b$ & \\
\hline & $0.90(\mathrm{~m})$ & $0.93-0.89(\mathrm{~m})$ & 0.01 \\
\hline \multirow[t]{2}{*}{23} & $1.41(\mathrm{~m})$ & $1.43-1.37(\mathrm{~m})$ & -0.01 \\
\hline & $0.94(\mathrm{~m})$ & $0.94-0.89(\mathrm{~m})$ & -0.02 \\
\hline 24 & $1.21(\mathrm{~m})$ & $1.24-1.16(\mathrm{~m})$ & -0.01 \\
\hline 25 & $1.56(\mathrm{~m})$ & $1.60-1.52(\mathrm{~m})$ & 0 \\
\hline 26 & $0.78(\mathrm{~d}, 6.8)$ & $0.78(\mathrm{~d}, 6.8)$ & 0 \\
\hline 27 & $0.85(\mathrm{~d}, 6.8)$ & $0.85(\mathrm{~d}, 6.8)$ & 0 \\
\hline 28 & $0.77(\mathrm{~d}, 6.8)$ & $0.77(\mathrm{~d}, 6.8)$ & 0 \\
\hline
\end{tabular}

${ }^{a}$ Chang, Y.-C.; Kuo, L.-M.; Su, J.-H.; Hwang, T.-L.; Kuo, Y.- H.; Lin, C.-S.; Wu, Y.-C.; Sheu, J.-H.; Sung, P.-J. Tetrahedron 2016, 72, 999.; Chang, Y.-C. Ph.D. Thesis, National Sun Yat-sen University, 2016. ${ }^{b}$ Chemical shifts are not assigned because the corresponding $\mathrm{C}-\mathrm{H}$ correlation peaks are not observed in the HSQC spectrum. 
${ }^{13} \mathrm{C}$ NMR data comparison of synthetic and natural pinnigorgiol $B$

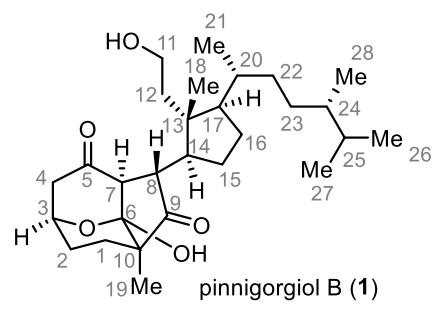

\begin{tabular}{|c|c|c|c|}
\hline Position & $\begin{array}{c}\text { Natural pinnigorgiol } \mathrm{B}^{\mathrm{a}} \\
\left(101 \mathrm{MHz} \text { in } \mathrm{CDCl}_{3}\right)\end{array}$ & $\begin{array}{c}\text { Synthetic pinnigorgiol B } \\
\left(101 \mathrm{MHz} \text { in } \mathrm{CDCl}_{3}\right)\end{array}$ & $\Delta \delta(\mathrm{ppm})$ \\
\hline 1 & 26.6 & 26.6 & 0 \\
\hline 2 & 24.1 & 24.1 & 0 \\
\hline 3 & 70.3 & 70.3 & 0 \\
\hline 4 & 43.9 & 43.9 & 0 \\
\hline 5 & 101.4 & 101.3 & -0.1 \\
\hline 6 & 208.1 & 208.0 & -0.1 \\
\hline 7 & 60.1 & 59.9 & -0.2 \\
\hline 8 & 47.5 & 47.51 & 0.01 \\
\hline 9 & 216.5 & 216.5 & 0 \\
\hline 10 & 50.0 & 50.0 & 0 \\
\hline 11 & 59.1 & 59.2 & 0.1 \\
\hline 12 & 39.7 & 39.6 & -0.1 \\
\hline 13 & 46.6 & 46.6 & 0 \\
\hline 14 & 47.5 & 47.44 & -0.06 \\
\hline 15 & 25.9 & 25.9 & 0 \\
\hline 16 & 24.3 & 24.2 & -0.1 \\
\hline 17 & 49.4 & 49.4 & 0 \\
\hline 18 & 17.1 & 17.1 & 0 \\
\hline 19 & 12.1 & 12.1 & 0 \\
\hline 20 & 33.4 & 33.3 & -0.1 \\
\hline 21 & 20.5 & 20.53 & 0.03 \\
\hline 22 & 32.7 & 32.6 & -0.1 \\
\hline 23 & 31.8 & 31.8 & 0 \\
\hline 24 & 39.0 & 39.0 & 0 \\
\hline 25 & 31.5 & 31.5 & 0 \\
\hline 26 & 17.6 & 17.6 & 0 \\
\hline 27 & 20.4 & 20.45 & 0.05 \\
\hline 28 & 15.4 & 15.4 & 0 \\
\hline
\end{tabular}

${ }^{a}$ Chang, Y.-C.; Kuo, L.-M.; Su, J.-H.; Hwang, T.-L.; Kuo, Y.- H.; Lin, C.-S.; Wu, Y.-C.; Sheu, J.-H.; Sung, P.-J. Tetrahedron 2016, 72, 999.; Chang, Y.-C. Ph.D. Thesis, National Sun Yat-sen University, 2016. 


\section{NMR spectra comparison of synthetic and natural pinnigorgiols $B$ and $E$}

${ }^{1} \mathrm{H}$ NMR spectra $(8.0 \sim 0.0 \mathrm{ppm})$ comparison of natural and synthetic pinnigorgiol $\mathrm{E}$

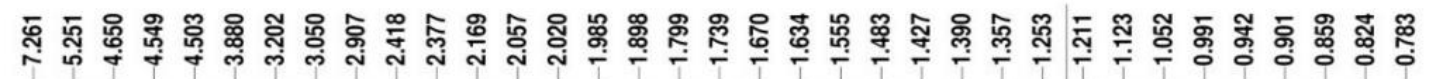
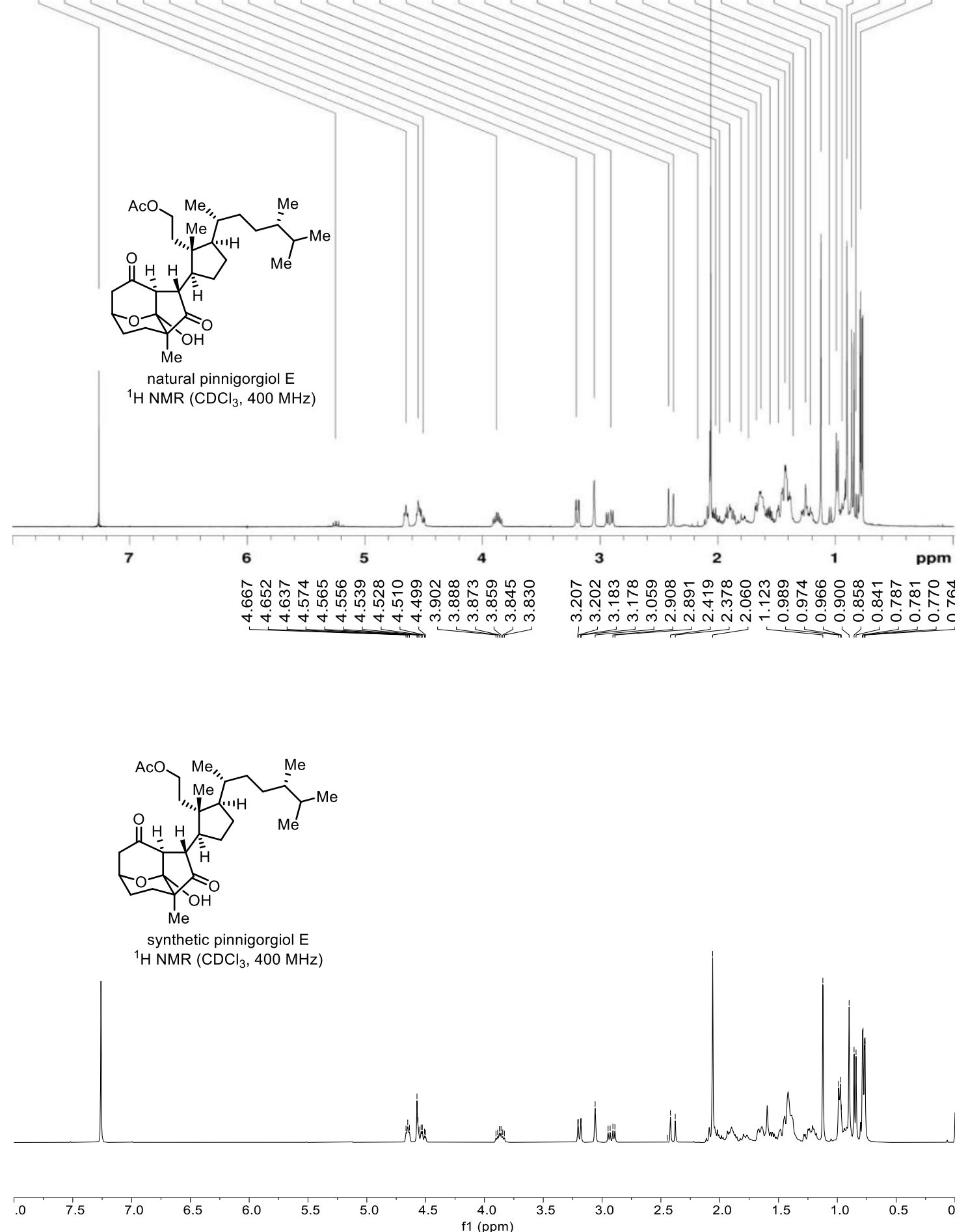
${ }^{1} \mathrm{H}$ NMR spectra (4.8 2.8 $\left.\mathrm{ppm}\right)$ comparison of natural and synthetic pinnigorgiol $\mathrm{E}$
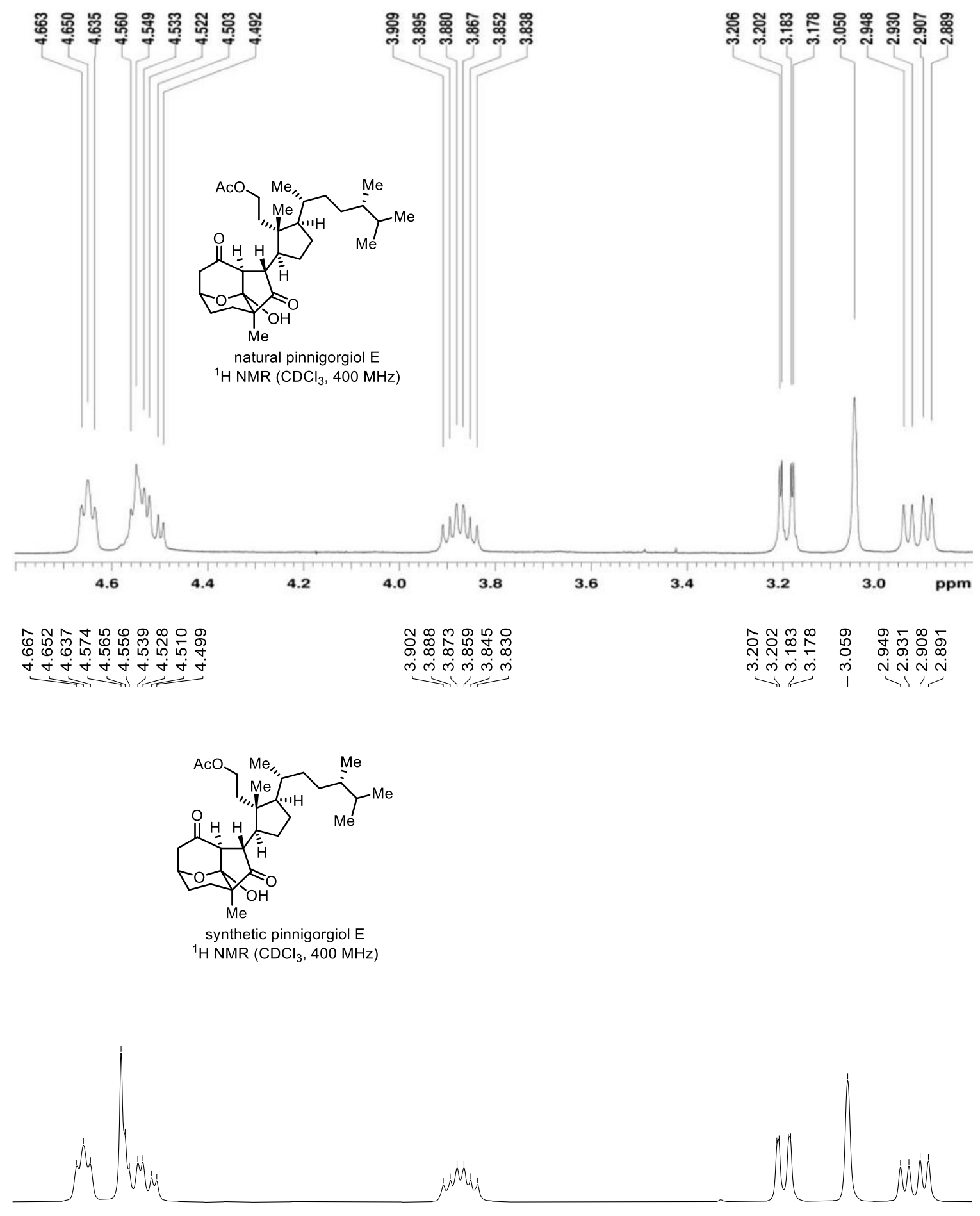

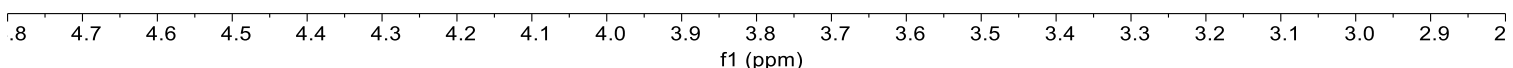


${ }^{1} \mathrm{H}$ NMR spectra (2.6 0.6 $\left.\mathrm{ppm}\right)$ comparison of natural and synthetic pinnigorgiol $\mathrm{E}$

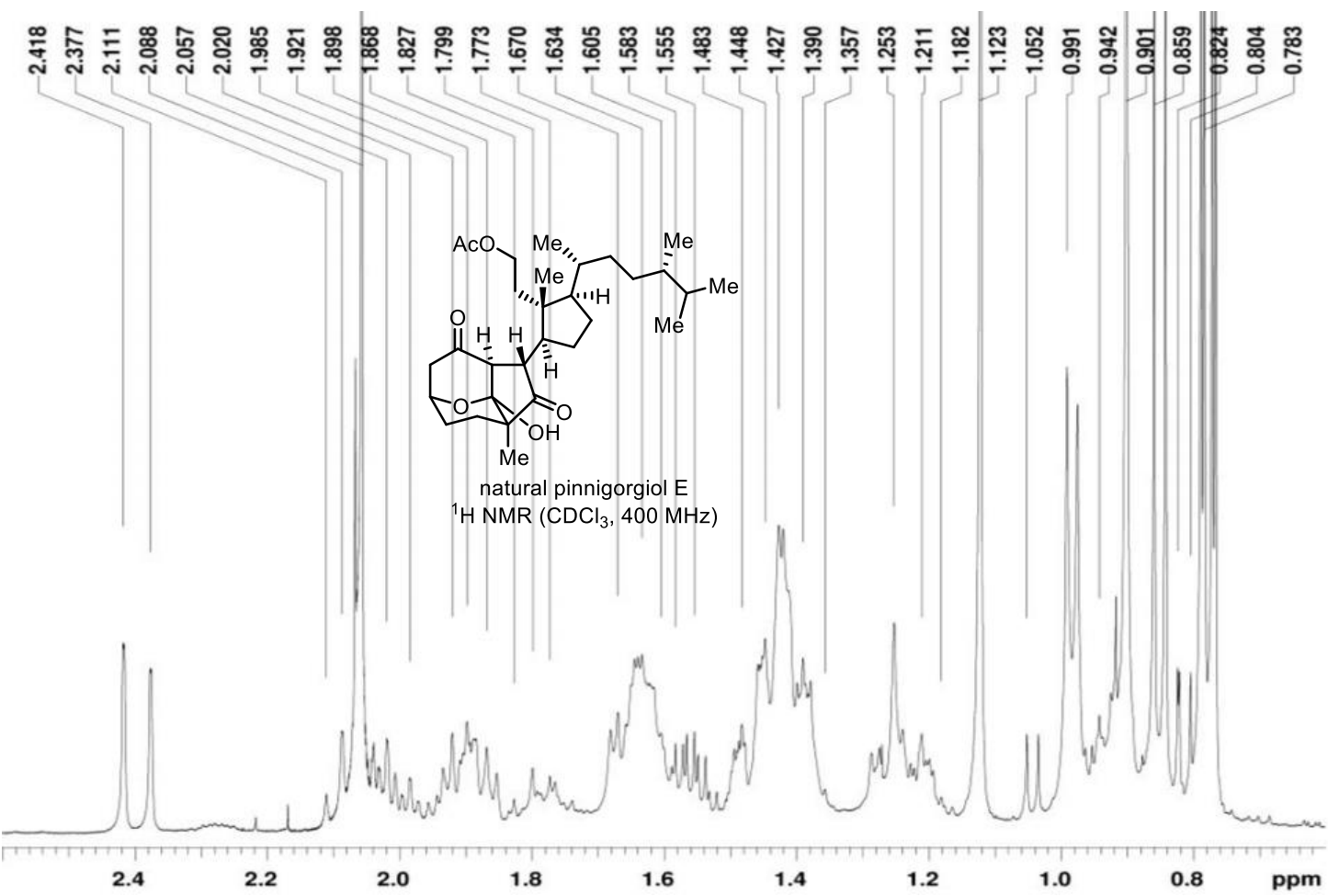

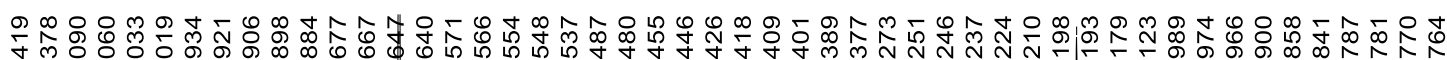

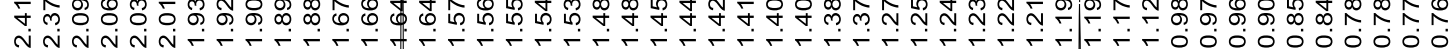

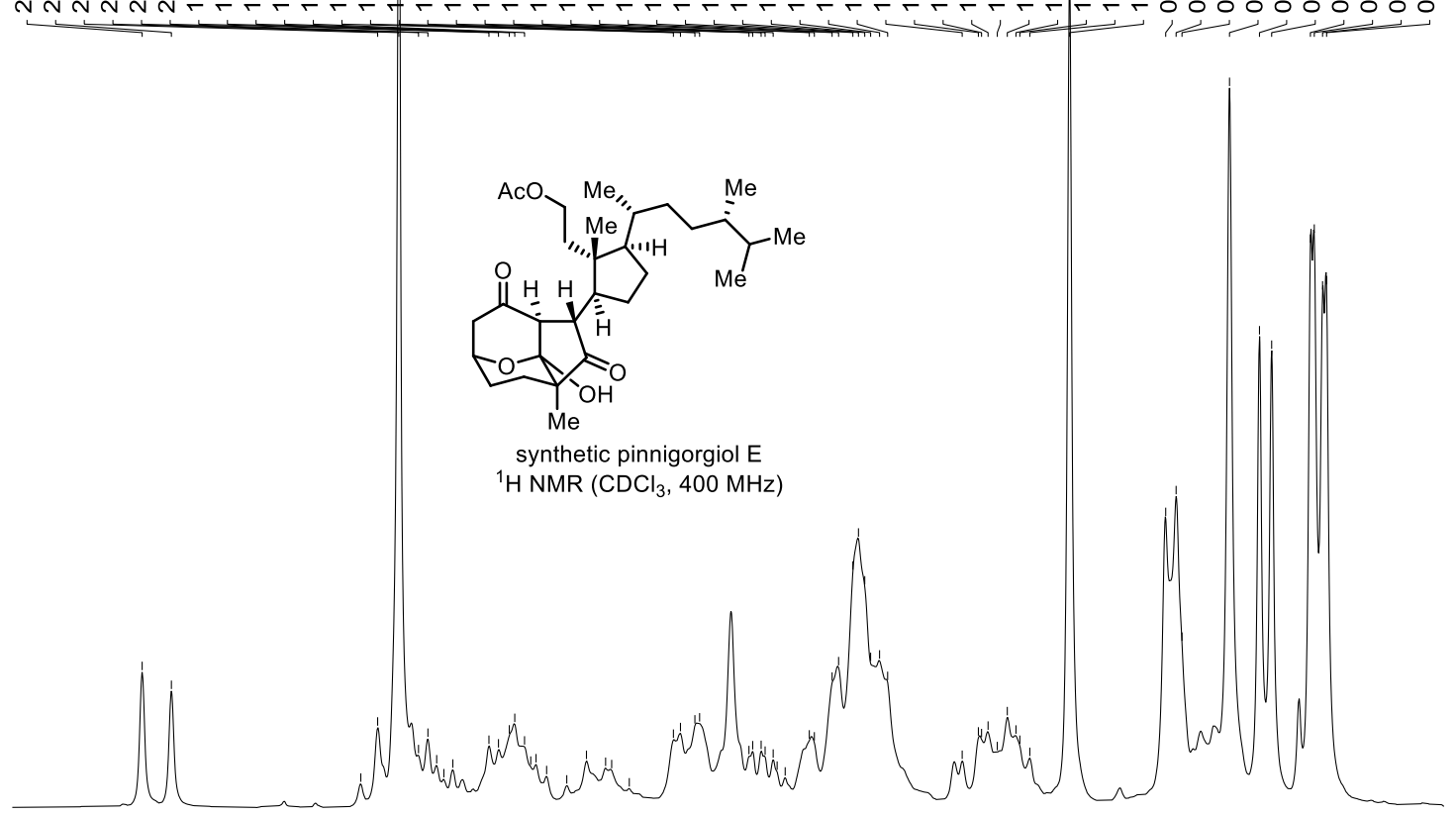

$\begin{array}{lllllllllll}2.5 & 2.4 & 2.3 & 2.2 & 2.1 & 2.0 & 1.9 & 1.8 & 1.7 & \begin{array}{l}1.6 \\ \mathrm{f} 1(\mathrm{ppm})\end{array} & 1.5\end{array}$ 


\section{${ }^{13} \mathrm{C}$ NMR spectra comparison of natural and synthetic pinnigorgiol $\mathrm{E}$}

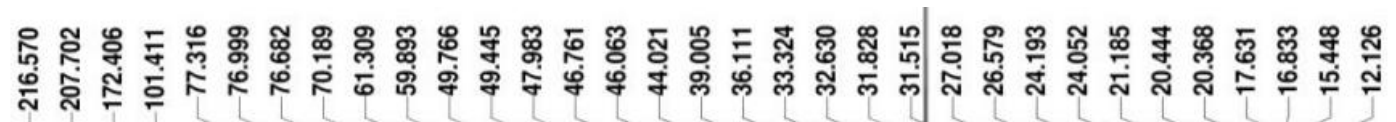

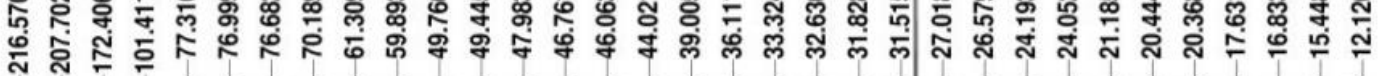

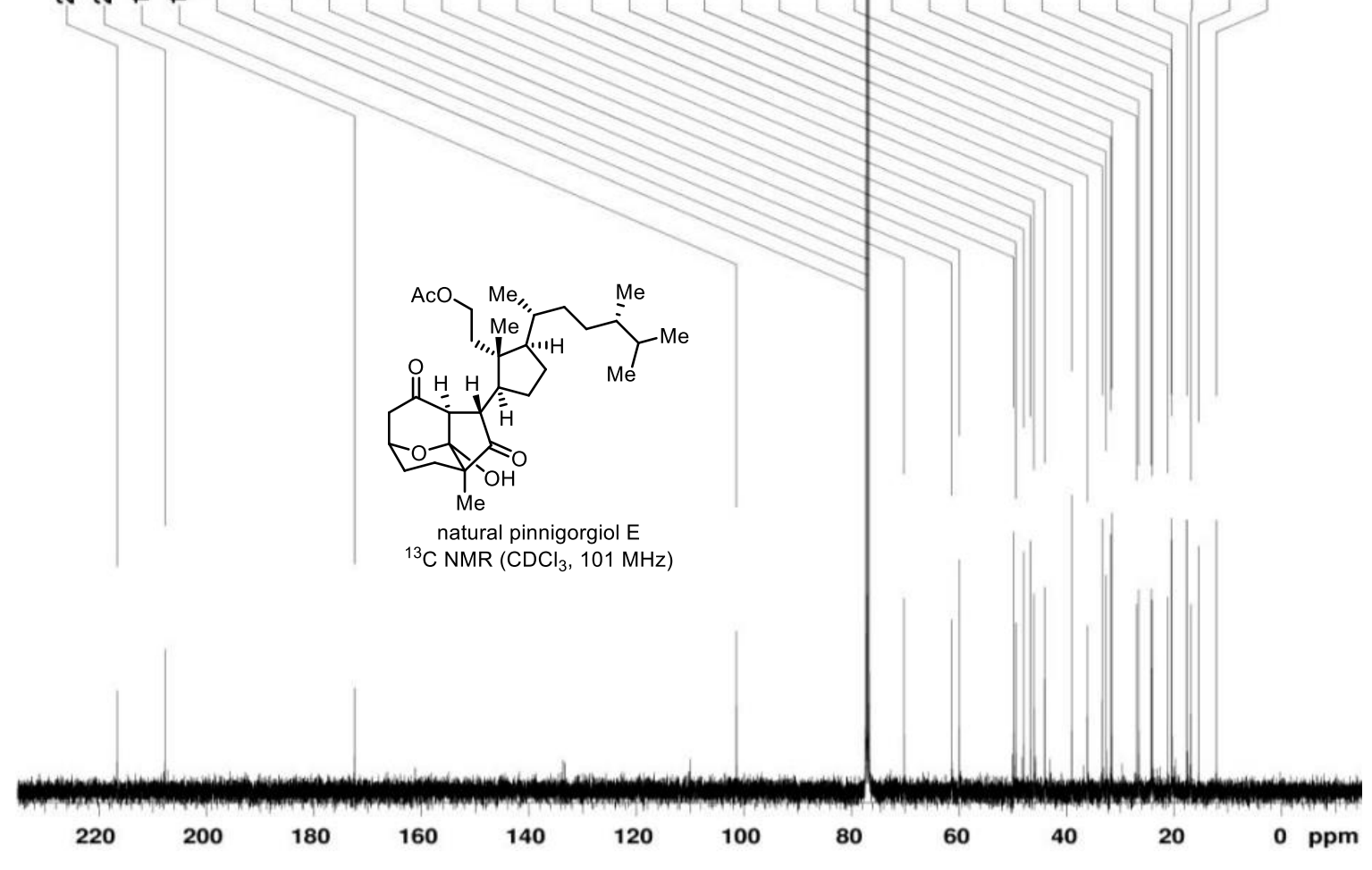

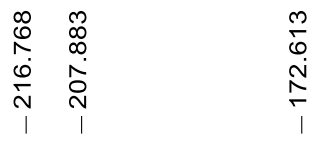

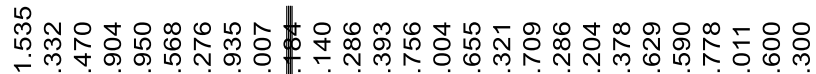

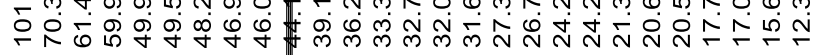

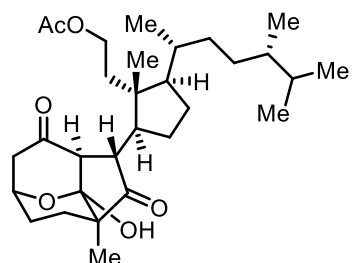

synthetic pinnigorgiol $\mathrm{E}$

${ }^{13} \mathrm{C} \mathrm{NMR}\left(\mathrm{CDCl}_{3}, 101 \mathrm{MHz}\right)$
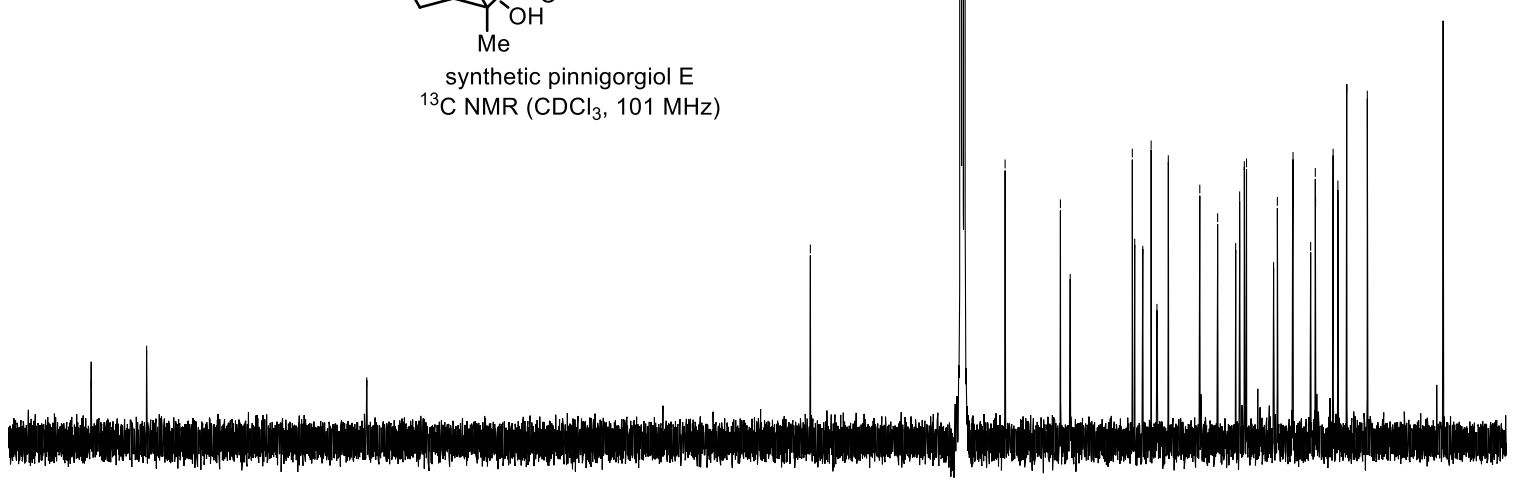

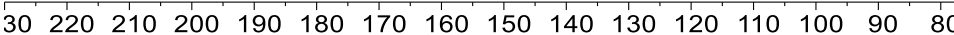

f1 (ppm) 
${ }^{1} \mathrm{H}$ NMR spectra $(8.0 \sim 0.0 \mathrm{ppm})$ comparison of natural and synthetic pinnigorgiol $B$

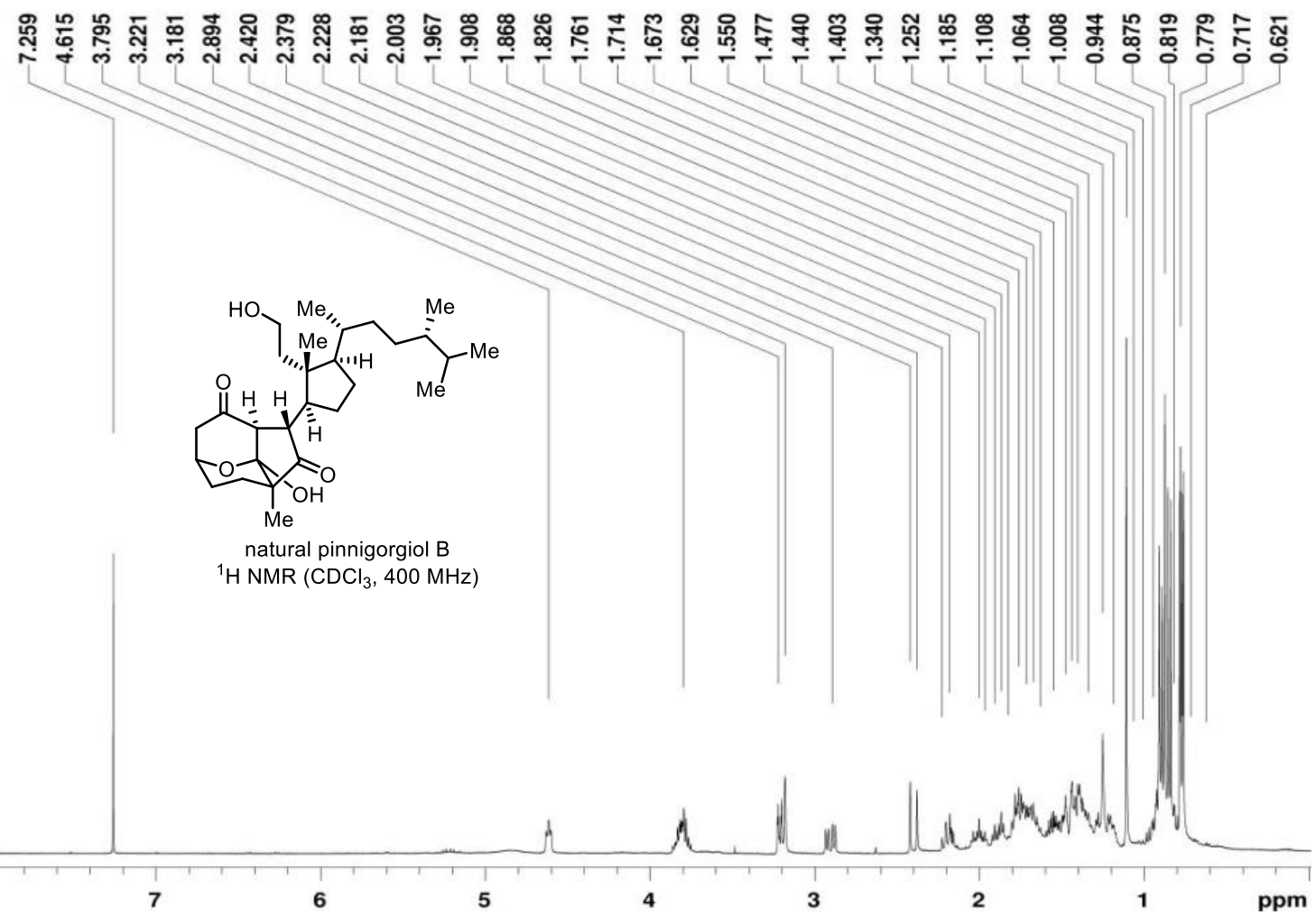

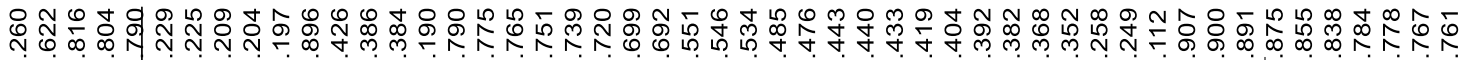

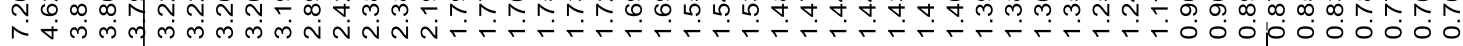

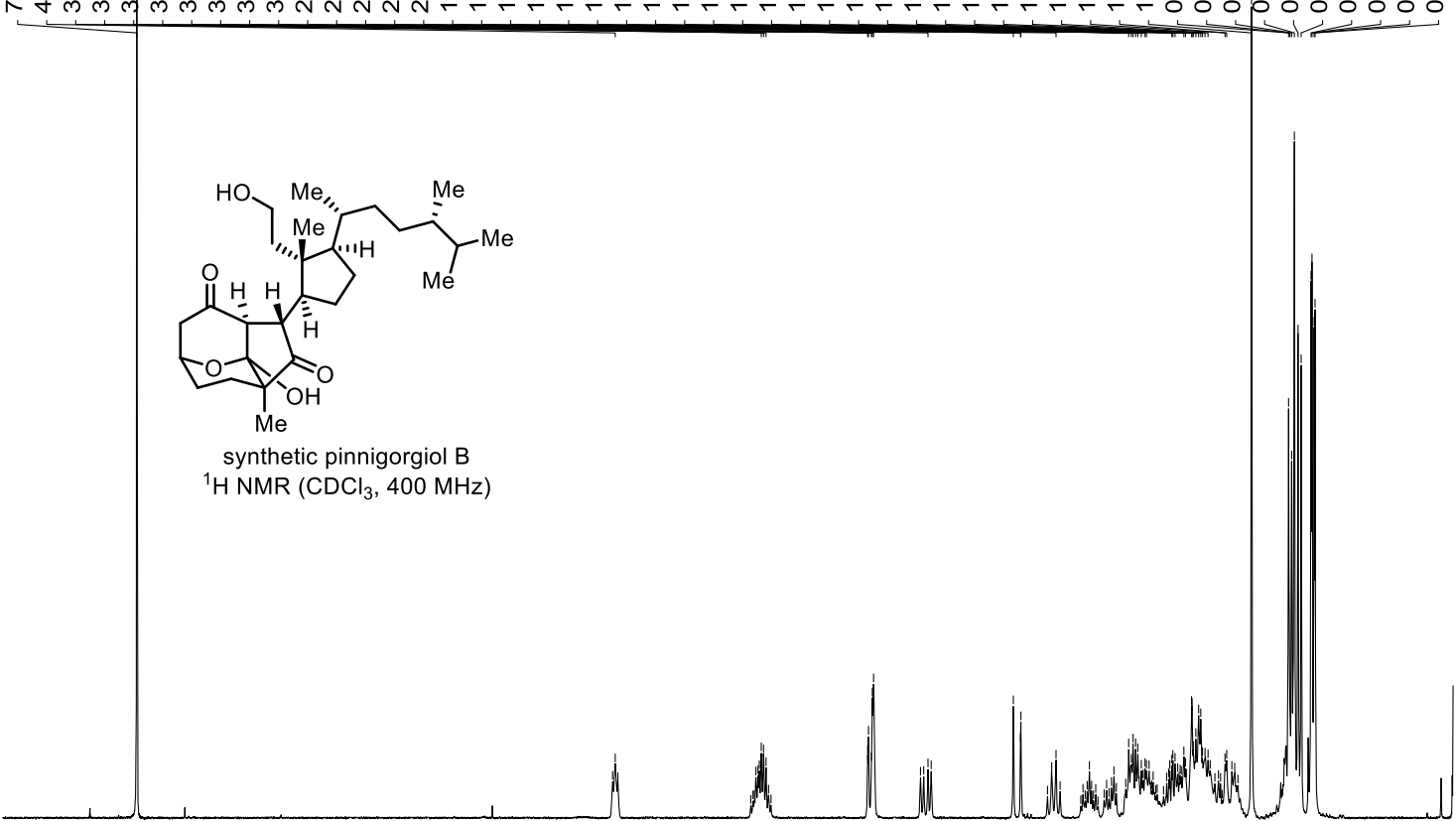

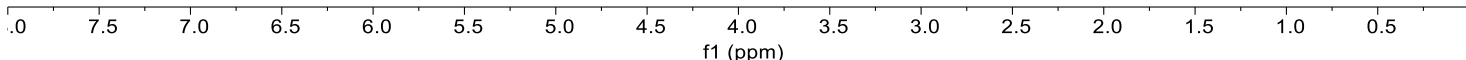


${ }^{1} \mathrm{H}$ NMR spectra (4.8 2.8 $\left.\mathrm{ppm}\right)$ comparison of natural and synthetic pinnigorgiol $B$
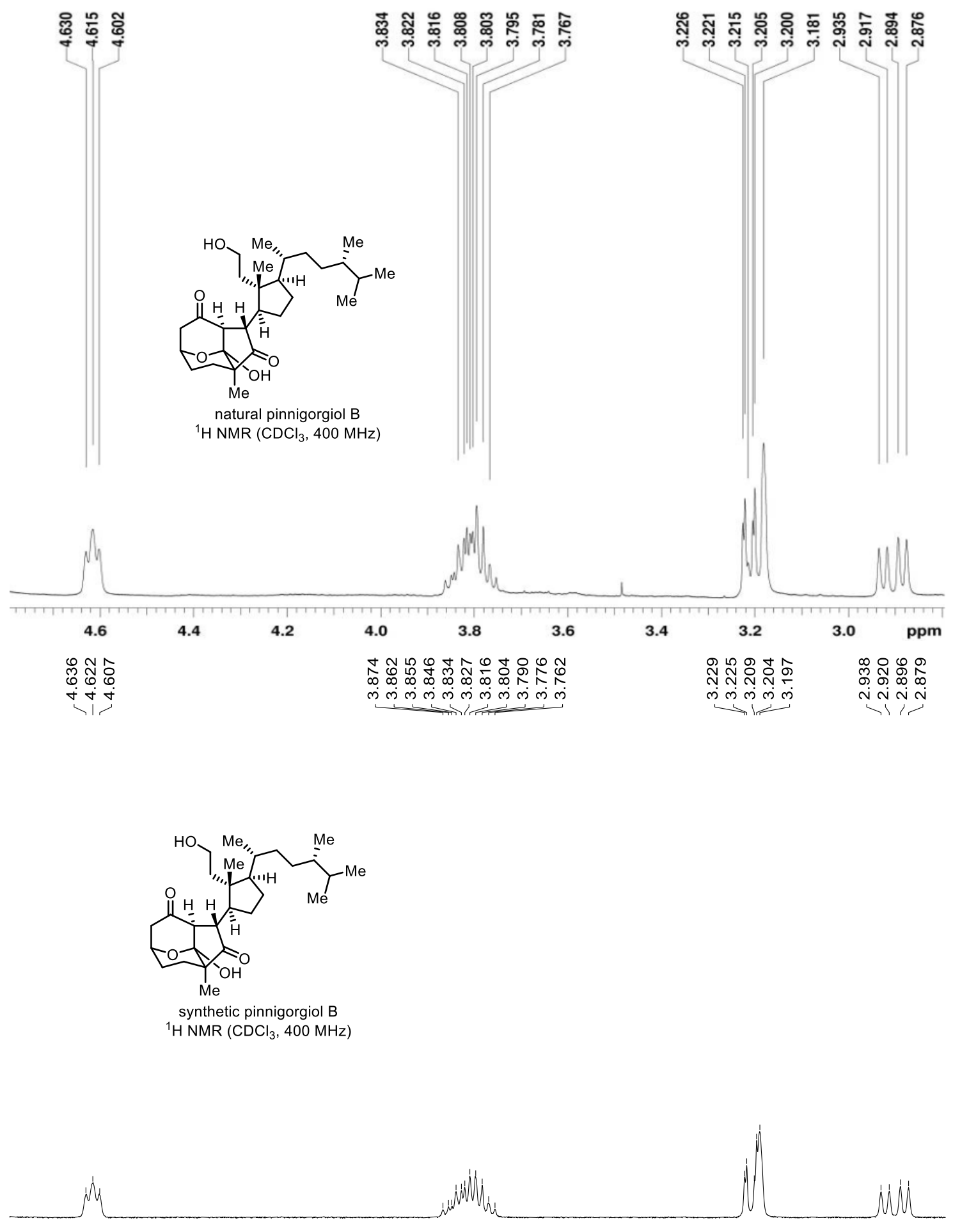

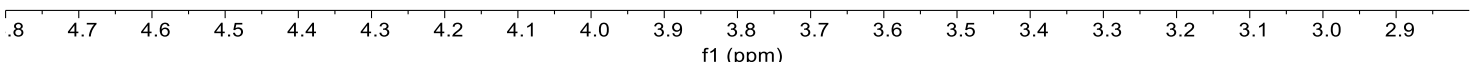


${ }^{1} \mathrm{H}$ NMR spectra (2.6 0.4 ppm) comparison of natural and synthetic pinnigorgiol $B$

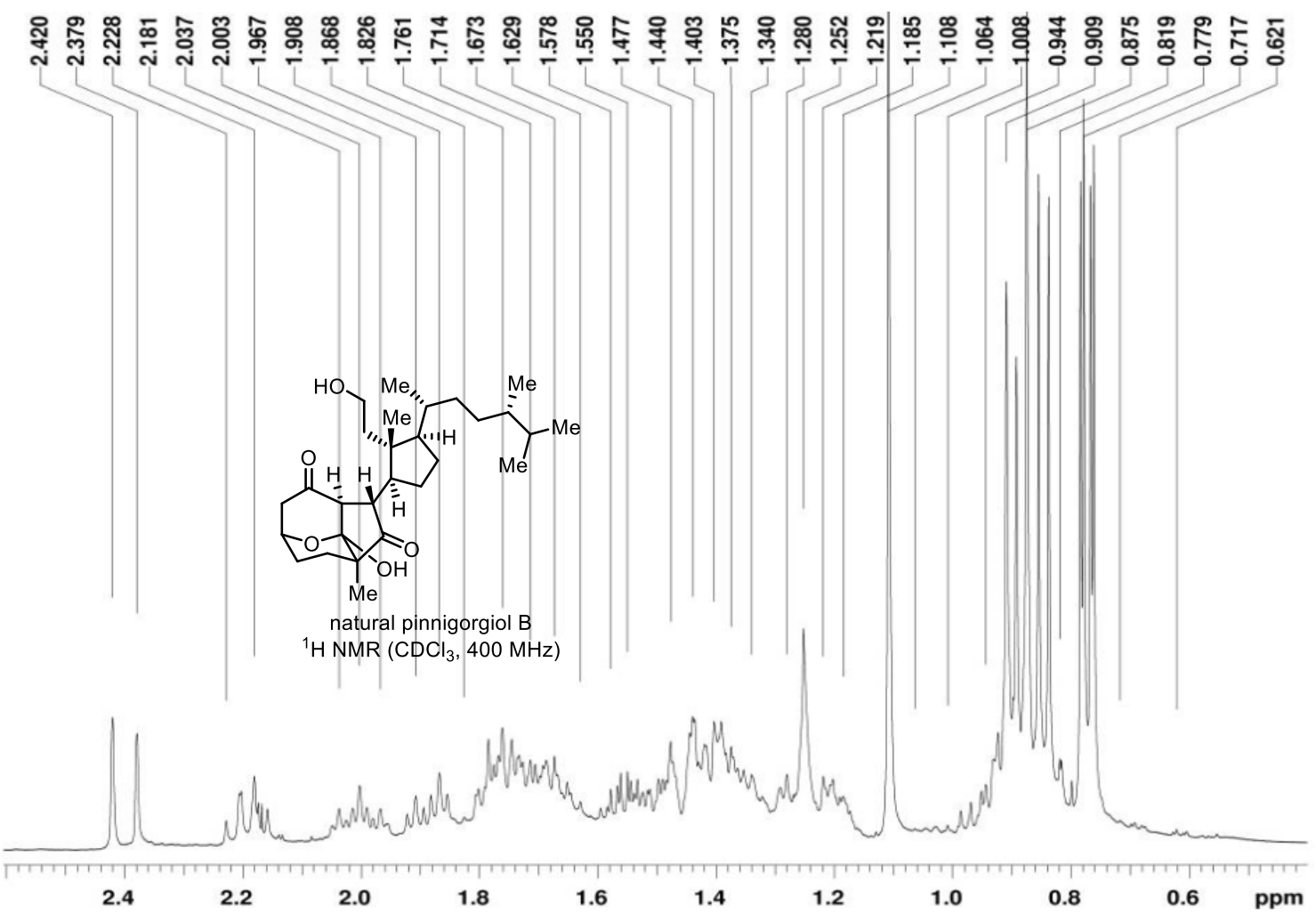

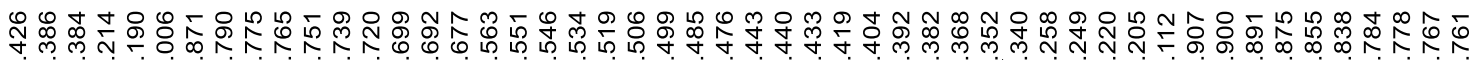

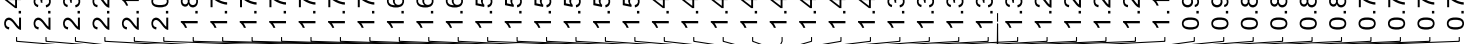

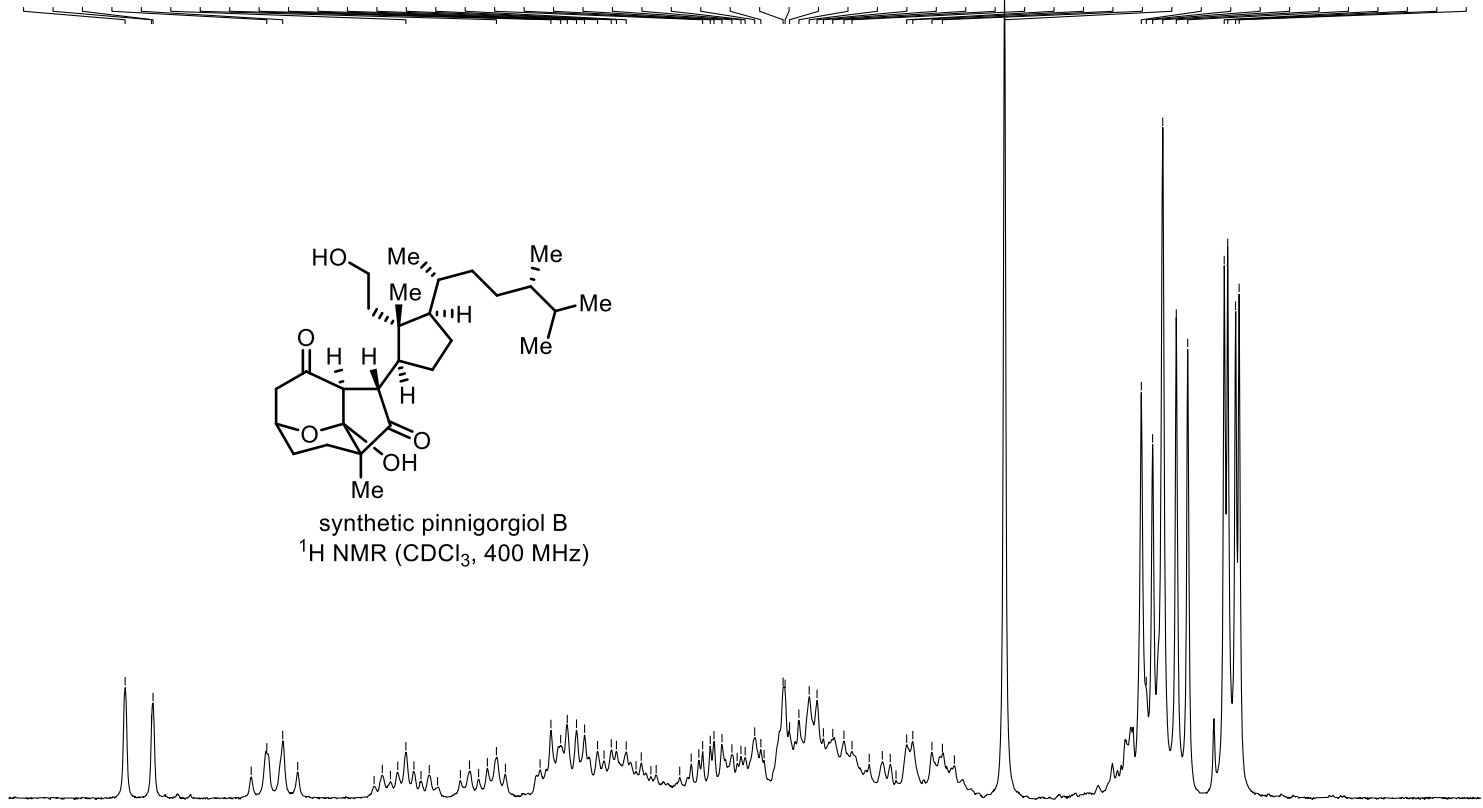

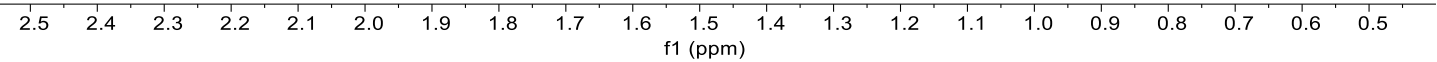


${ }^{13} \mathrm{C}$ NMR spectra comparison of natural and synthetic pinnigorgiol $B$

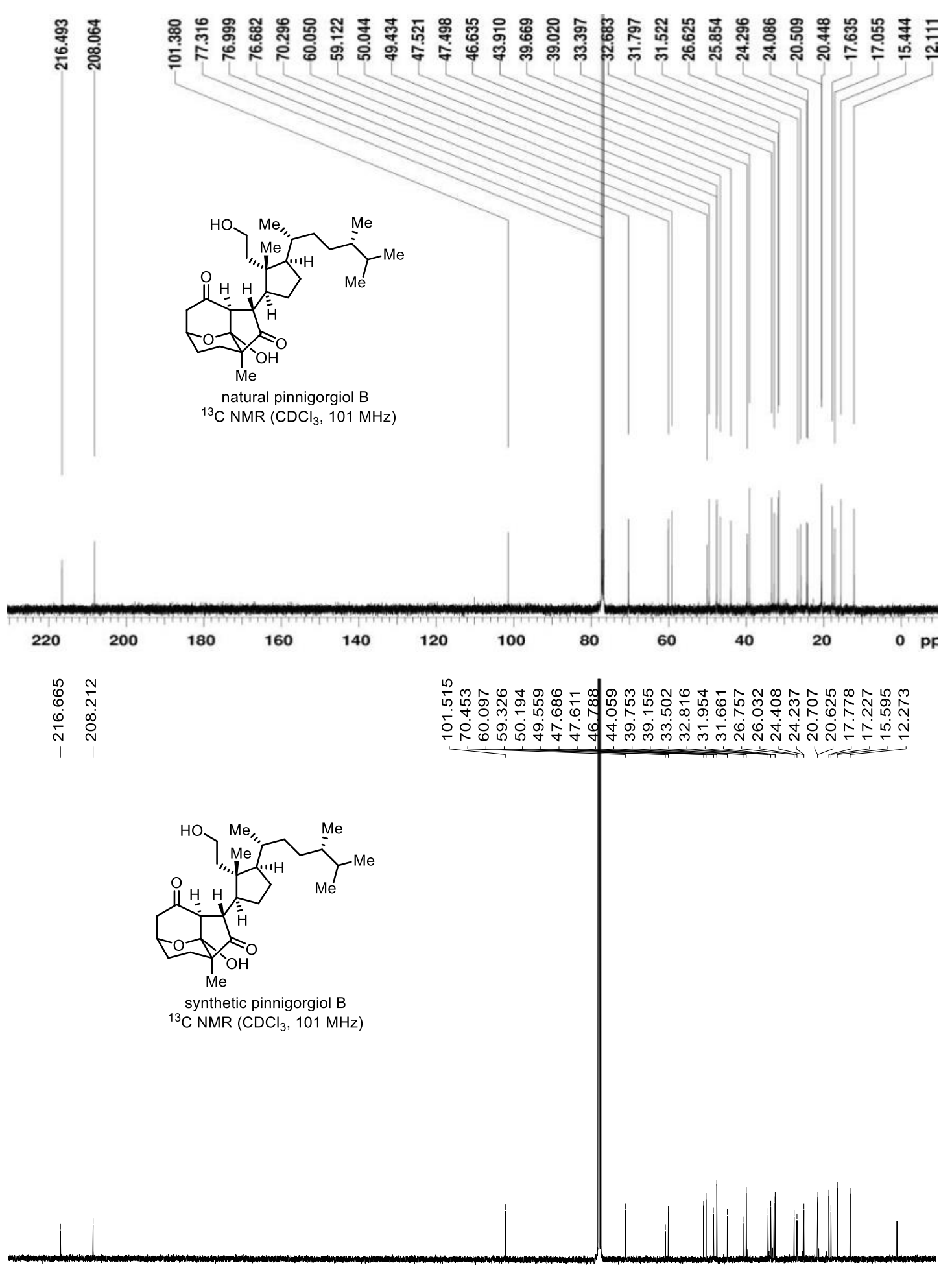

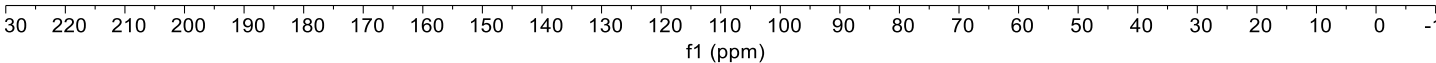




\section{ECD spectra of natural and synthetic pinnigorgiols $B$ and E}

1) sample name: Synthetic pinnigorgiol B

concentration: $\mathrm{C}=1.0 \mathrm{mg} / \mathrm{mL}$ in $\mathrm{MeOH}$

wavelength range: $200-400 \mathrm{~nm}$

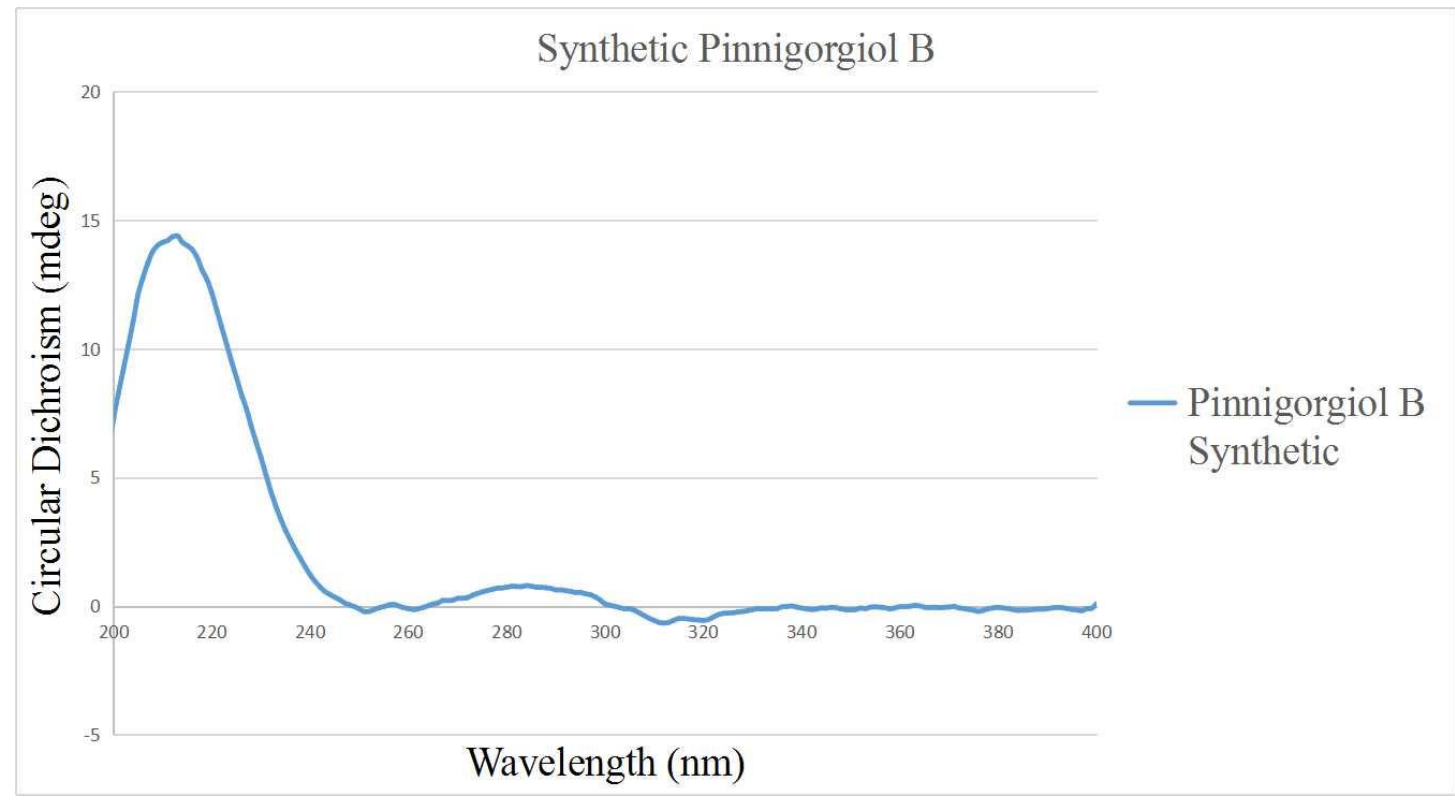

\section{Natural pinnigorgiol B:}

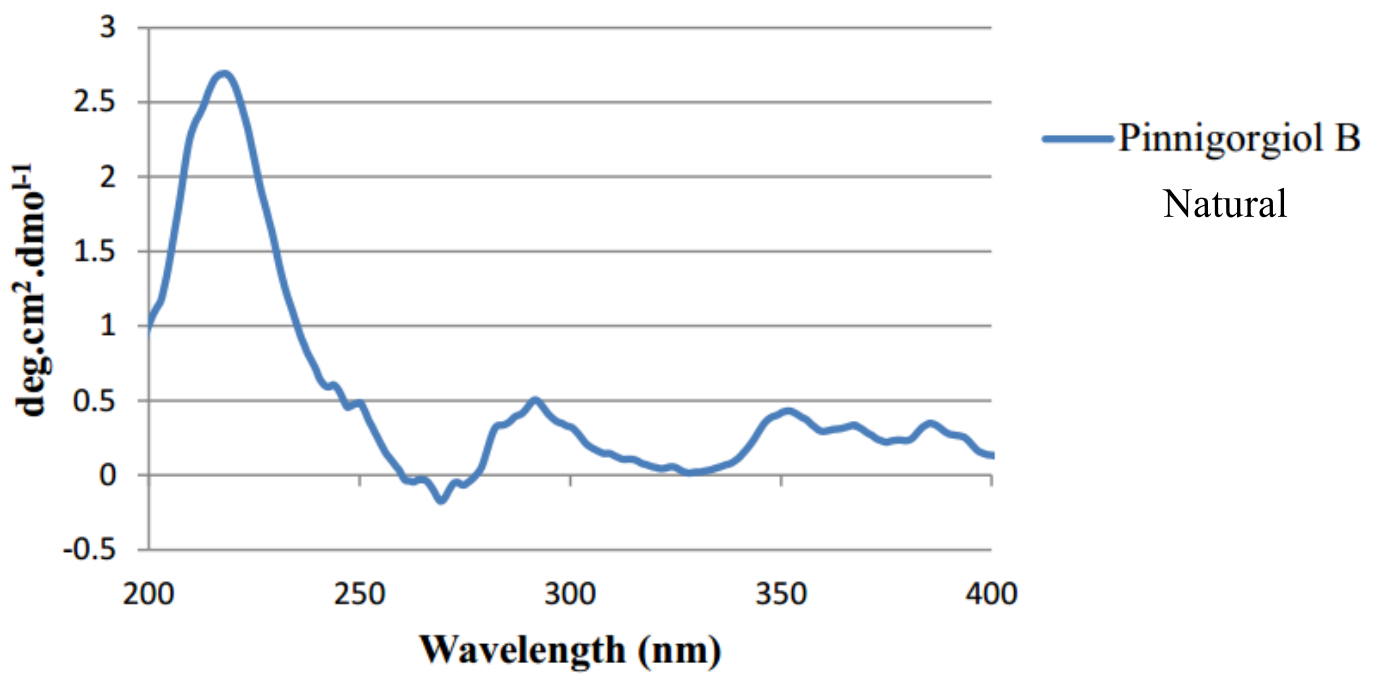


2) sample name: Synthetic pinnigorgiol $\mathbf{E}$

concentration: $\mathrm{C}=2.0 \mathrm{mg} / \mathrm{mL}$ in $\mathrm{MeOH}$

wavelength range: $200-400 \mathrm{~nm}$

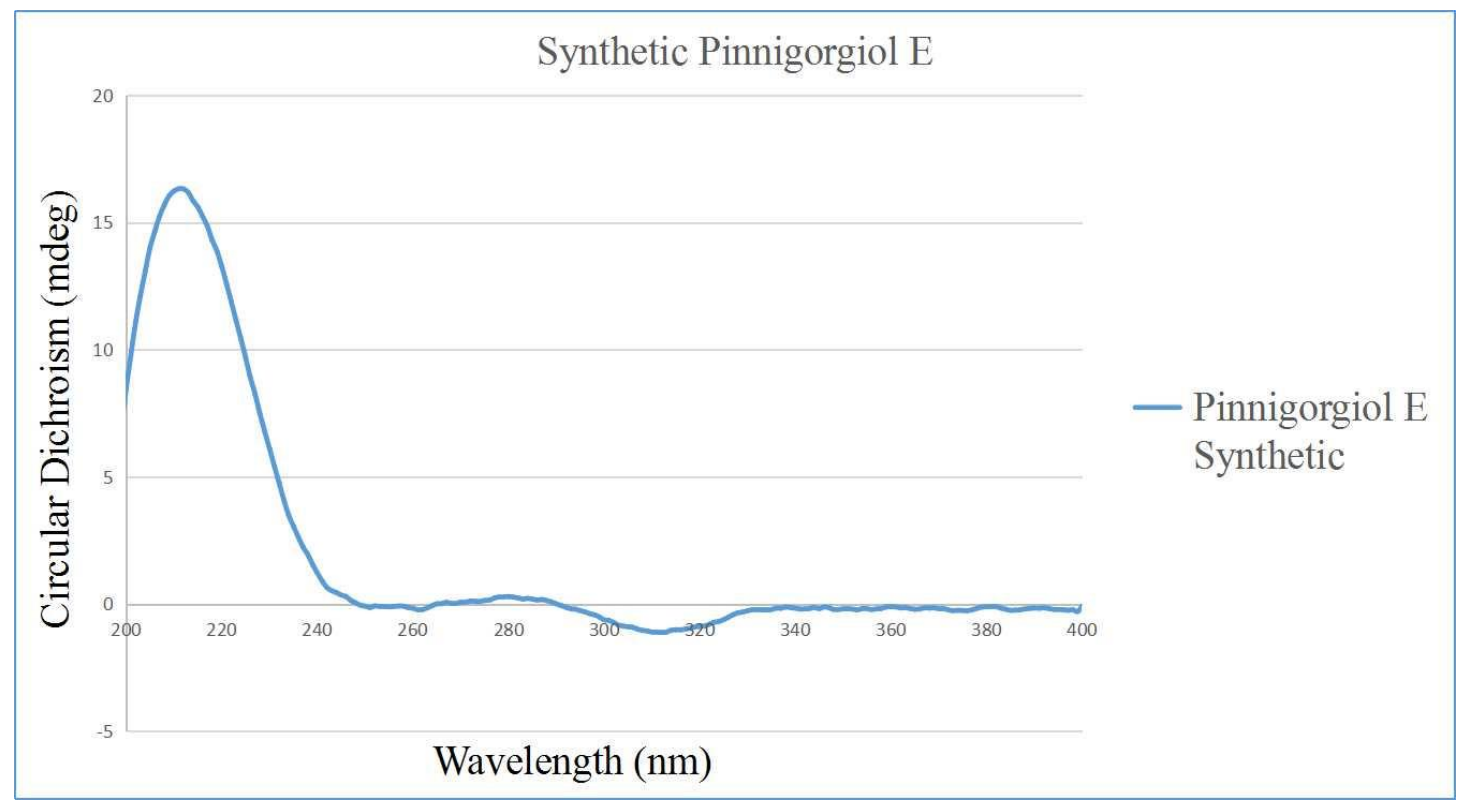

\section{Natural pinnigorgiol E:}

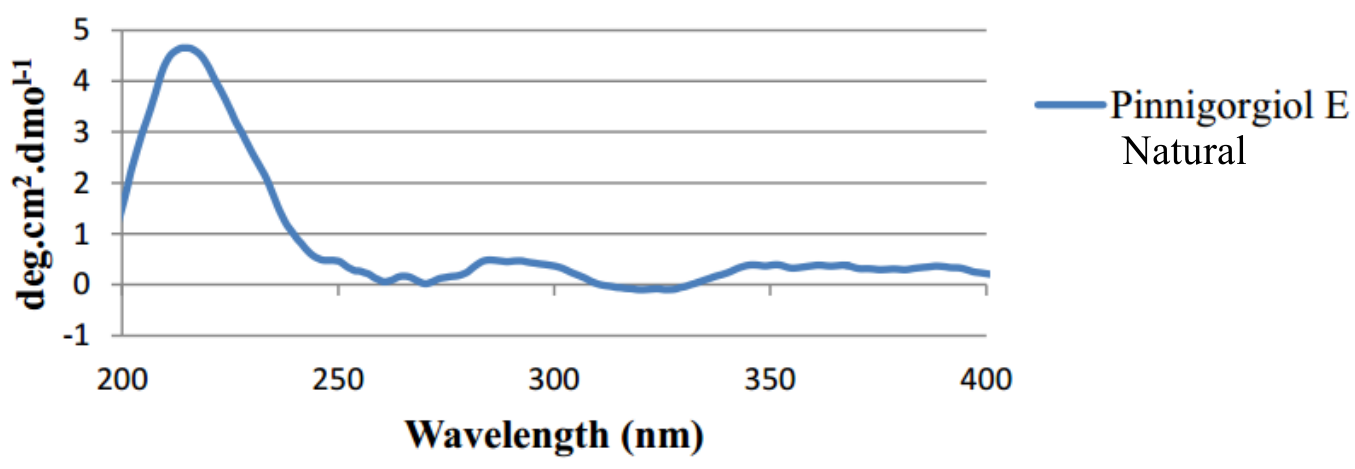




\section{X-ray crystallographic data for compounds S8 and S10}
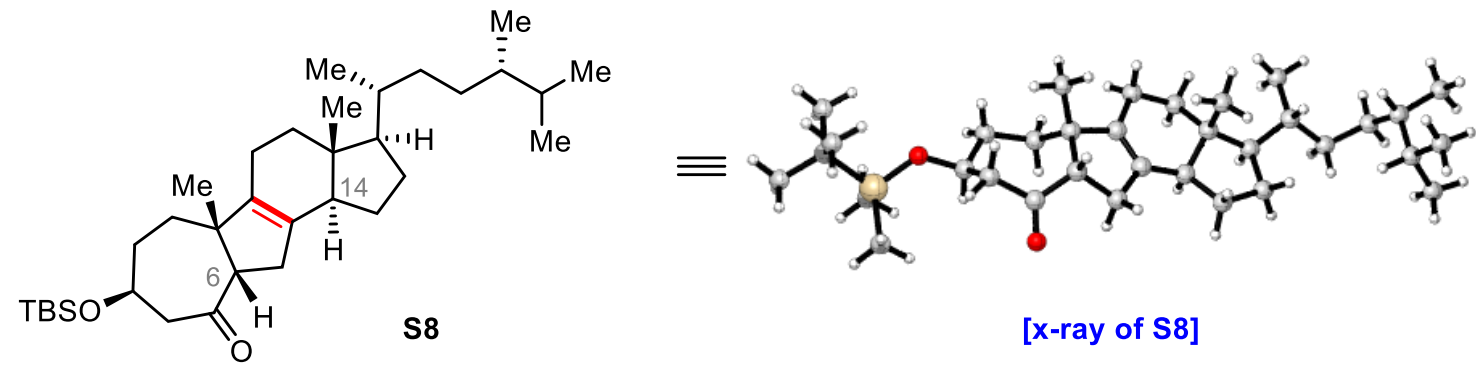

Table S1. Crystal data and structure refinement for CCDC 2058214

Identification code

Empirical formula

Formula weight

Temperature

Wavelength

Crystal system

Space group

Unit cell dimensions

Volume

$\mathrm{Z}$

Density (calculated)

Absorption coefficient

$\mathrm{F}(000)$

Crystal size

Theta range for data collection

Index ranges

Reflections collected

Independent reflections

Completeness to theta $=25.242^{\circ}$

Absorption correction

Max. and min. transmission

Refinement method

Data / restraints / parameters
CCDC 2058214

C34 H60 O2 Si

528.91

193(2) K

$0.71073 \AA$

Orthorhombic

P 212121

$\begin{array}{ll}\mathrm{a}=7.4082(2) \AA & \alpha=90^{\circ} . \\ \mathrm{b}=9.6115(3) \AA & \beta=90^{\circ} . \\ \mathrm{c}=47.4056(11) \AA & \gamma=90^{\circ} .\end{array}$

3375.46(16) $\AA^{3}$

4

$1.041 \mathrm{Mg} / \mathrm{m}^{3}$

$0.095 \mathrm{~mm}^{-1}$

1176

$0.170 \times 0.150 \times 0.110 \mathrm{~mm}^{3}$

1.718 to $26.000^{\circ}$.

$-9<=\mathrm{h}<=9,-11<=\mathrm{k}<=10,-58<=1<=58$

30593

$6601[\mathrm{R}(\mathrm{int})=0.0543]$

$99.8 \%$

Semi-empirical from equivalents

0.7456 and 0.6795

Full-matrix least-squares on $\mathrm{F}^{2}$

6601 / 0 / 346 
Goodness-of-fit on $\mathrm{F}^{2}$

Final $\mathrm{R}$ indices [I $>2 \operatorname{sigma}(\mathrm{I})]$

$\mathrm{R}$ indices (all data)

Absolute structure parameter

Extinction coefficient

Largest diff. peak and hole
1.034

$\mathrm{R} 1=0.0566, \mathrm{wR} 2=0.1410$

$\mathrm{R} 1=0.0826, \mathrm{wR} 2=0.1627$

$0.05(7)$

$0.015(3)$

0.363 and -0.210 e. $\AA^{-3}$ 

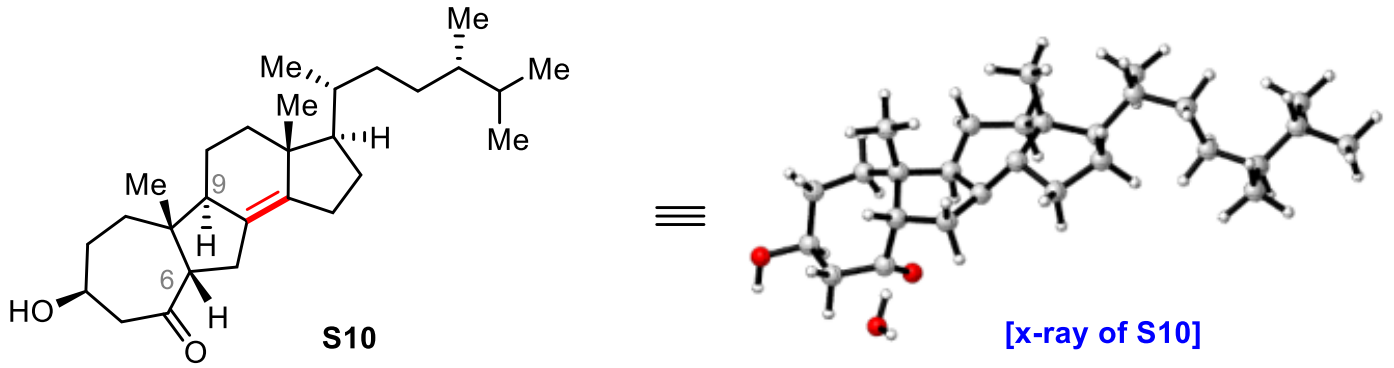

Table S2. Crystal data and structure refinement for CCDC 2058215

Identification code

Empirical formula

Formula weight

Temperature

Wavelength

Crystal system

Space group

Unit cell dimensions

Volume

Z

Density (calculated)

Absorption coefficient

$\mathrm{F}(000)$

Crystal size

Theta range for data collection

Index ranges

Reflections collected

Independent reflections

Completeness to theta $=67.679^{\circ}$

Absorption correction

Max. and min. transmission

Refinement method

Data / restraints / parameters

Goodness-of-fit on $\mathrm{F}^{2}$
CCDC 2058215

C26 H44 O3

404.61

293(2) K

$1.54178 \AA$

Monoclinic

P 21

$\mathrm{a}=6.6874(3) \AA$ $\alpha=90^{\circ}$.

$\mathrm{b}=7.5234(3) \AA$ $\beta=93.274(2)^{\circ}$.

$\mathrm{c}=27.1638(11) \AA$ $\gamma=90^{\circ}$.

2

$0.985 \mathrm{Mg} / \mathrm{m}^{3}$

$0.481 \mathrm{~mm}^{-1}$

448

$0.170 \times 0.140 \times 0.070 \mathrm{~mm}^{3}$

6.529 to $66.462^{\circ}$.

$-7<=\mathrm{h}<=7,-8<=\mathrm{k}<=8,-32<=\mathrm{l}<=32$

19832

$4730[\mathrm{R}(\mathrm{int})=0.0647]$

$96.4 \%$

Semi-empirical from equivalents

0.7456 and 0.5608

Full-matrix least-squares on $\mathrm{F}^{2}$

$4730 / 75 / 348$

1.044 
Final $\mathrm{R}$ indices [I $>2 \operatorname{sigma}(\mathrm{I})]$

$\mathrm{R}$ indices (all data)

Absolute structure parameter

Extinction coefficient

Largest diff. peak and hole
$\mathrm{R} 1=0.0615, \mathrm{wR} 2=0.1631$

$\mathrm{R} 1=0.0756, \mathrm{wR} 2=0.1836$

0.05(16)

0.057(8)

0.255 and -0.266 e. $\AA^{-3}$ 
9. NMR spectra

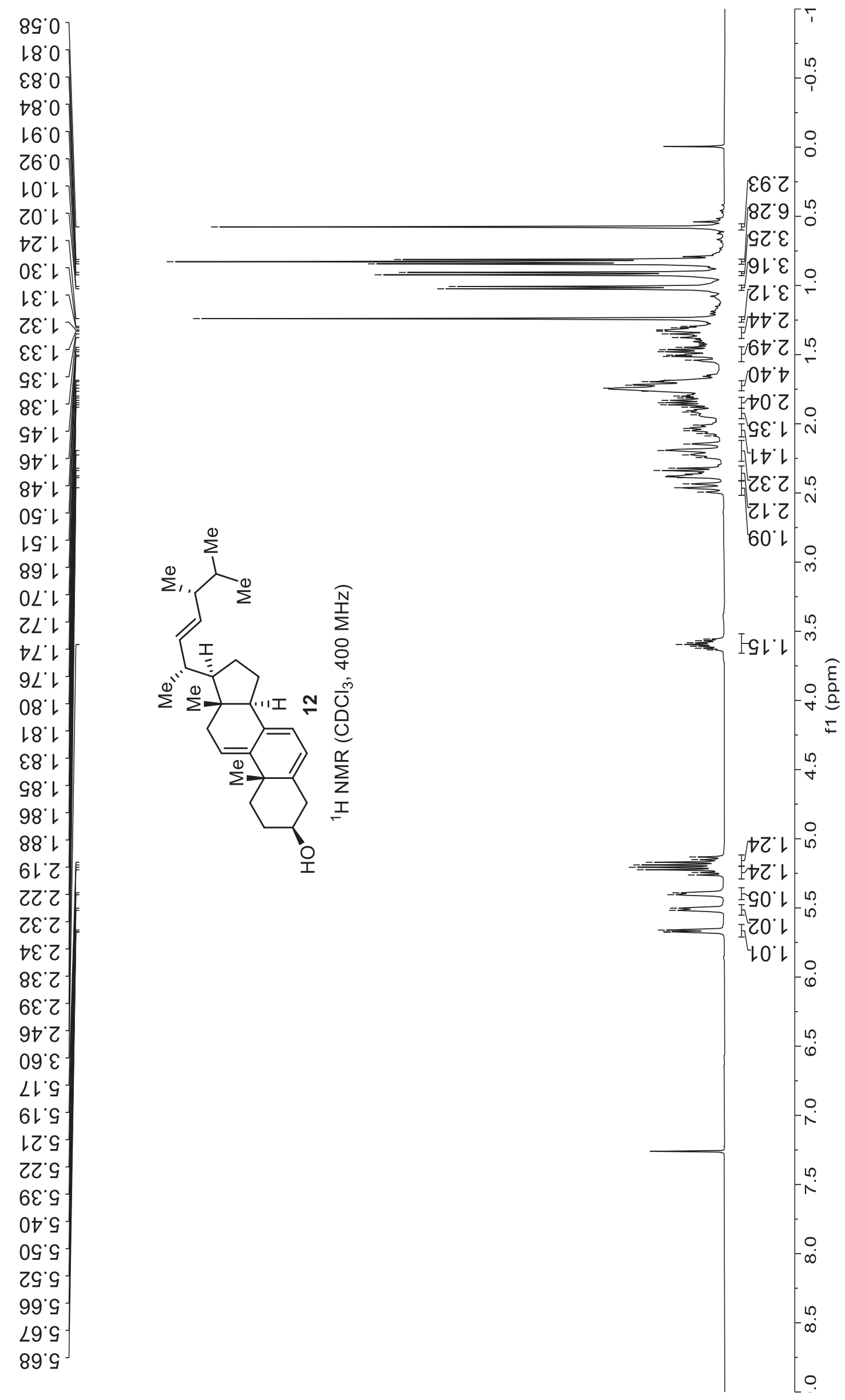



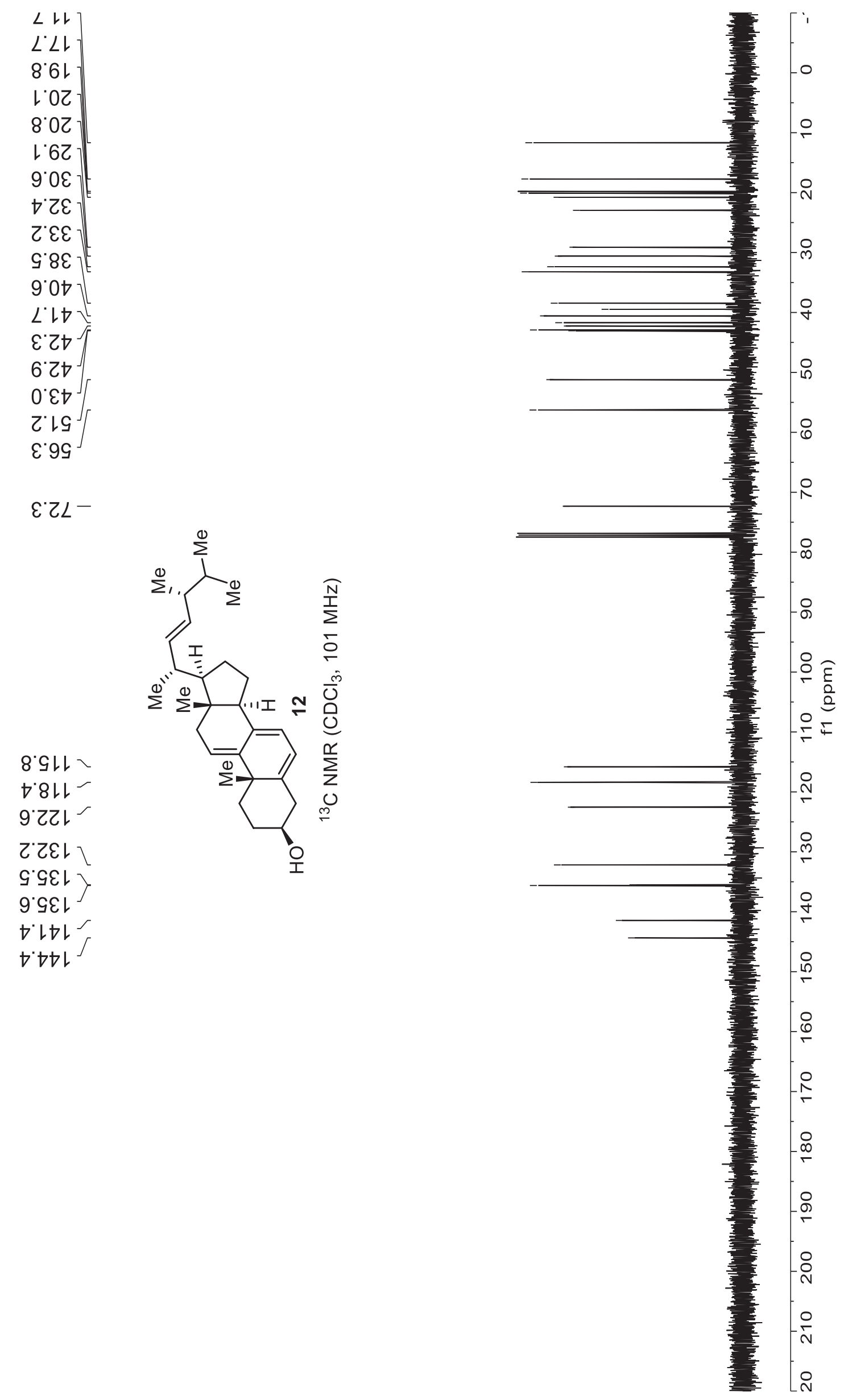


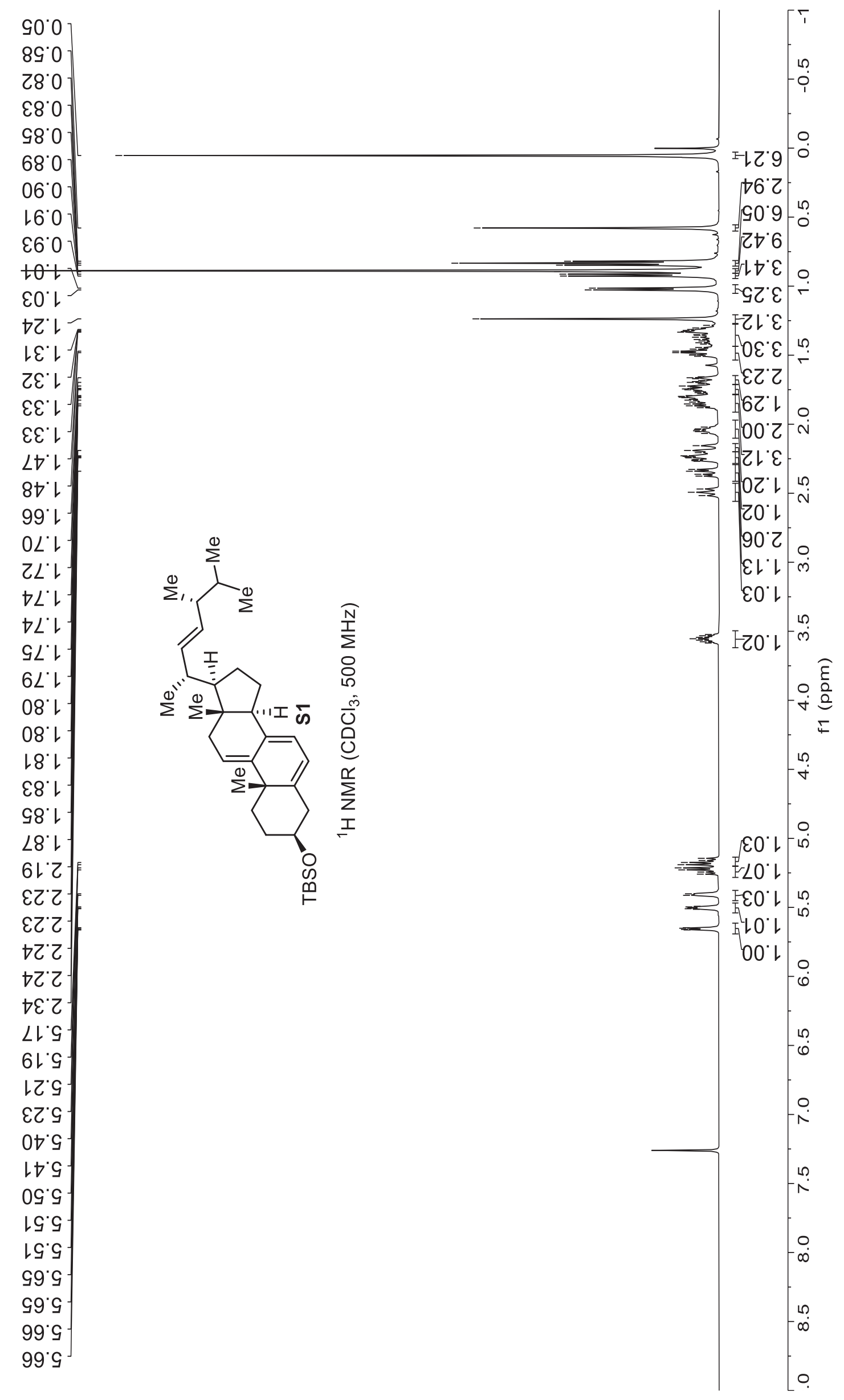




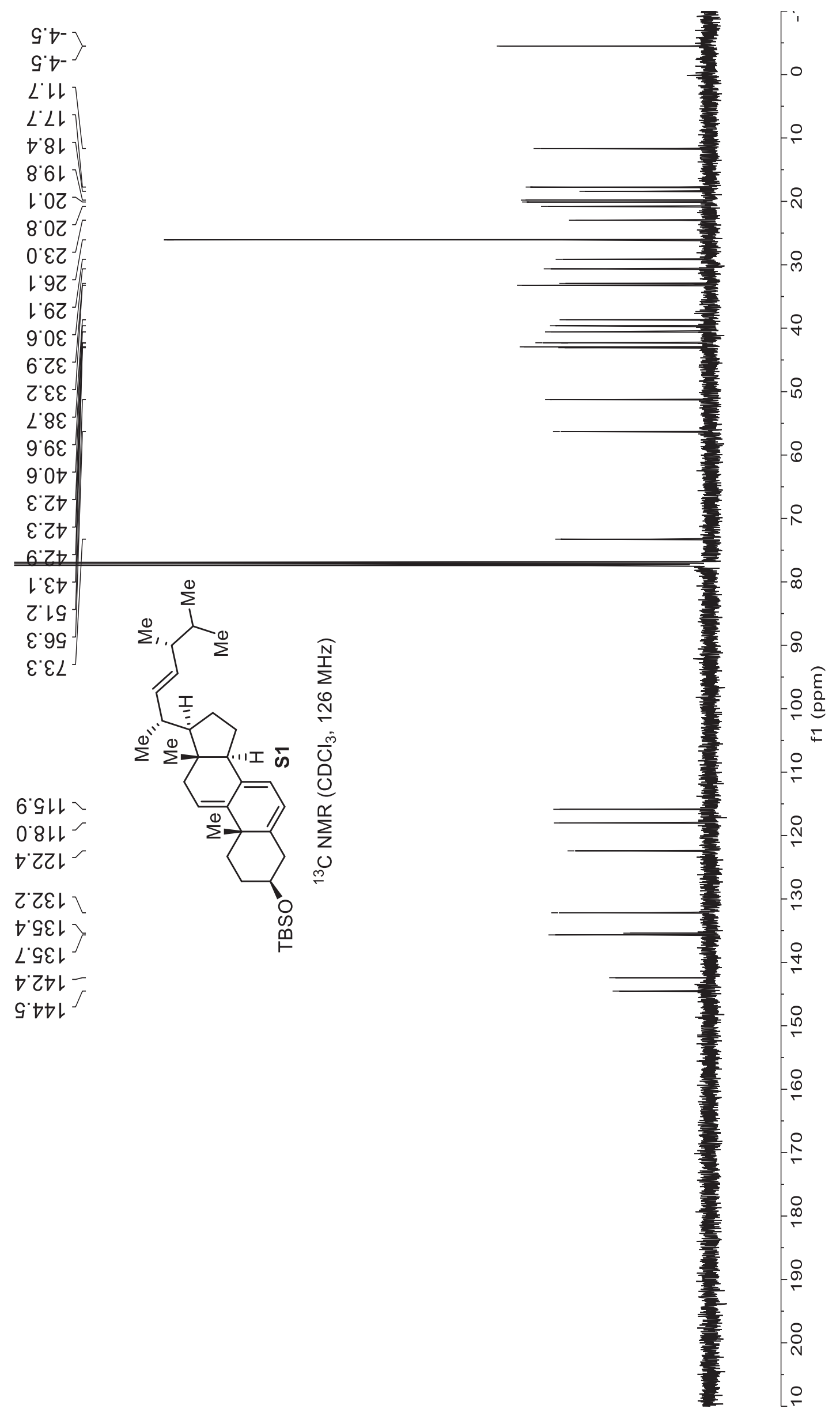




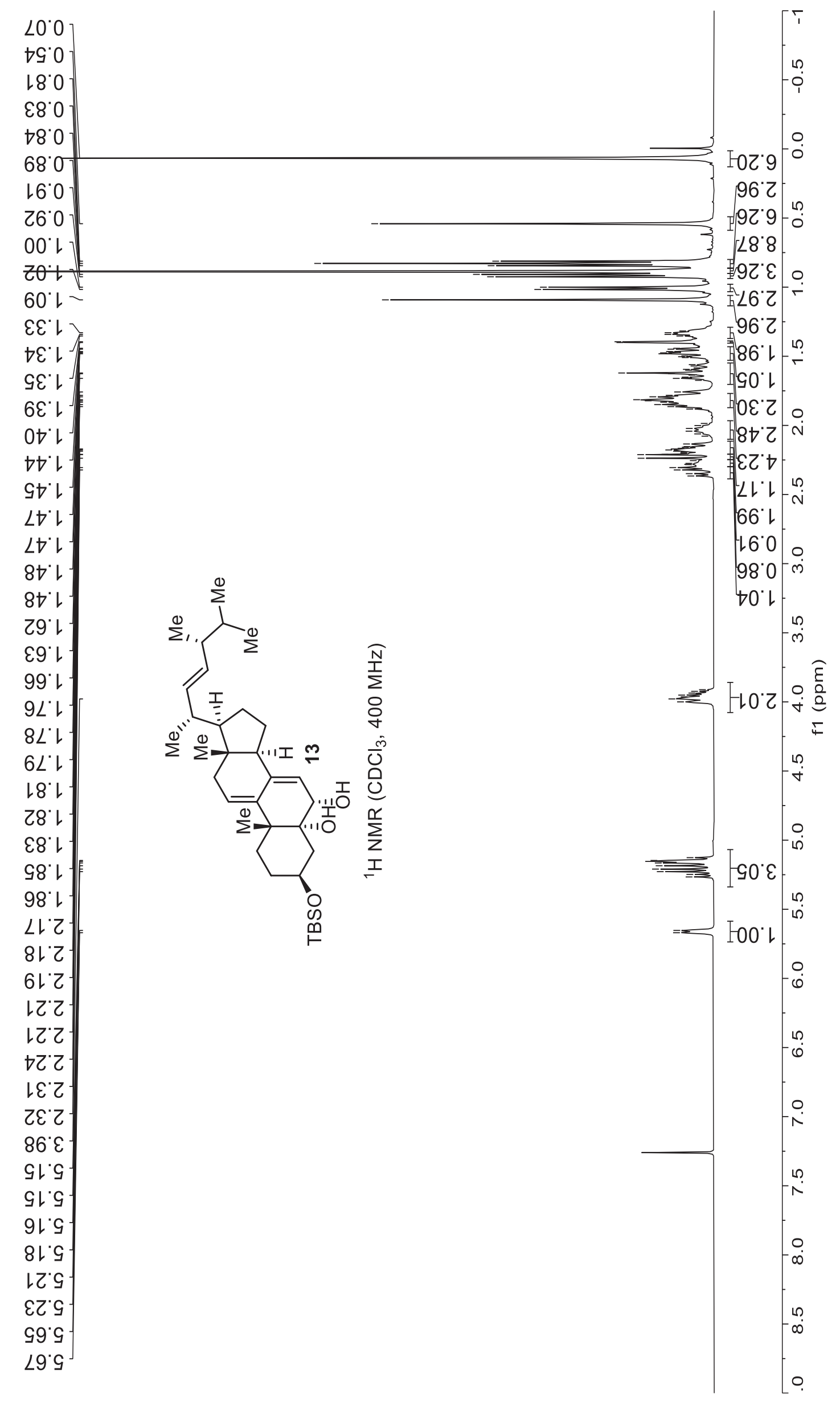




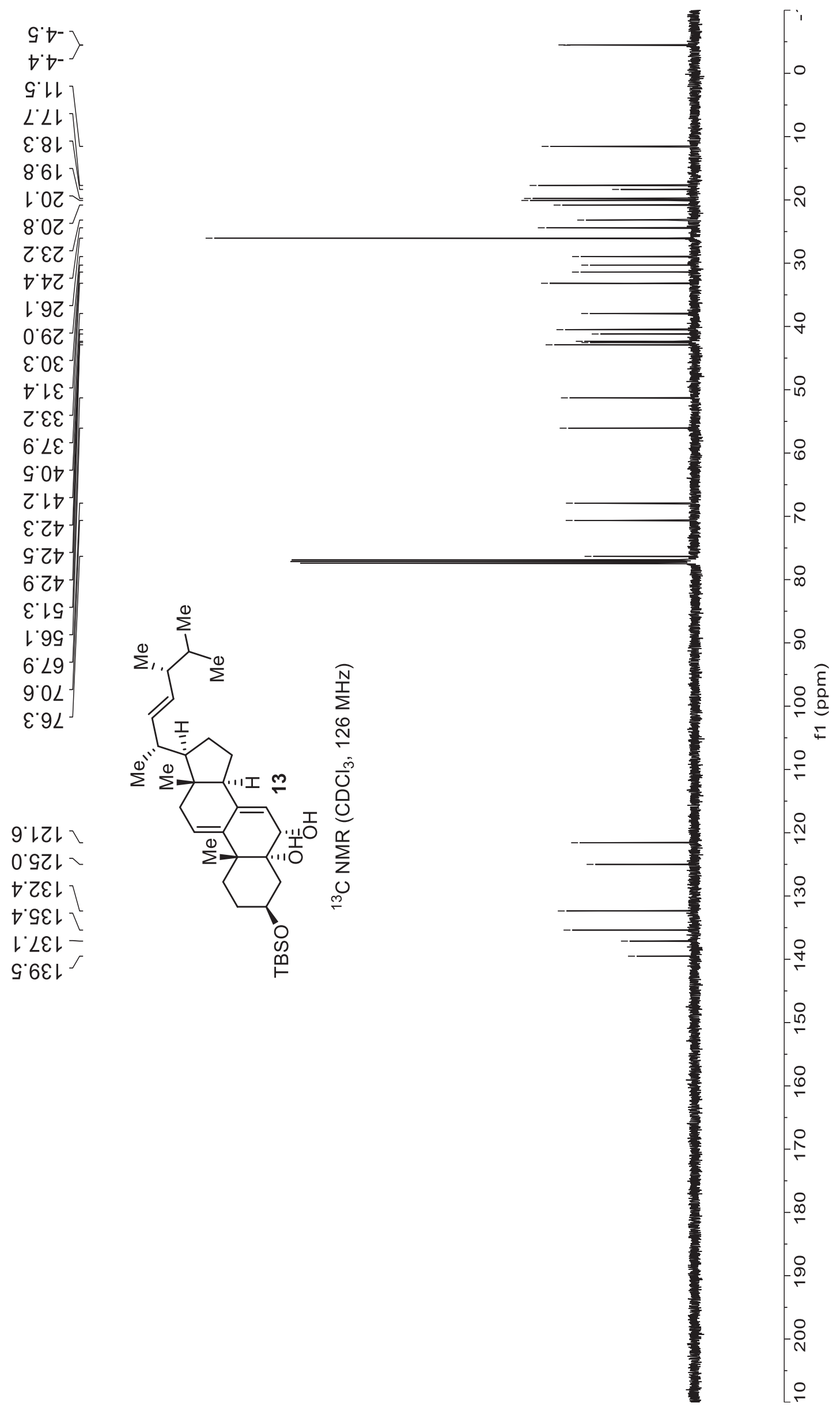




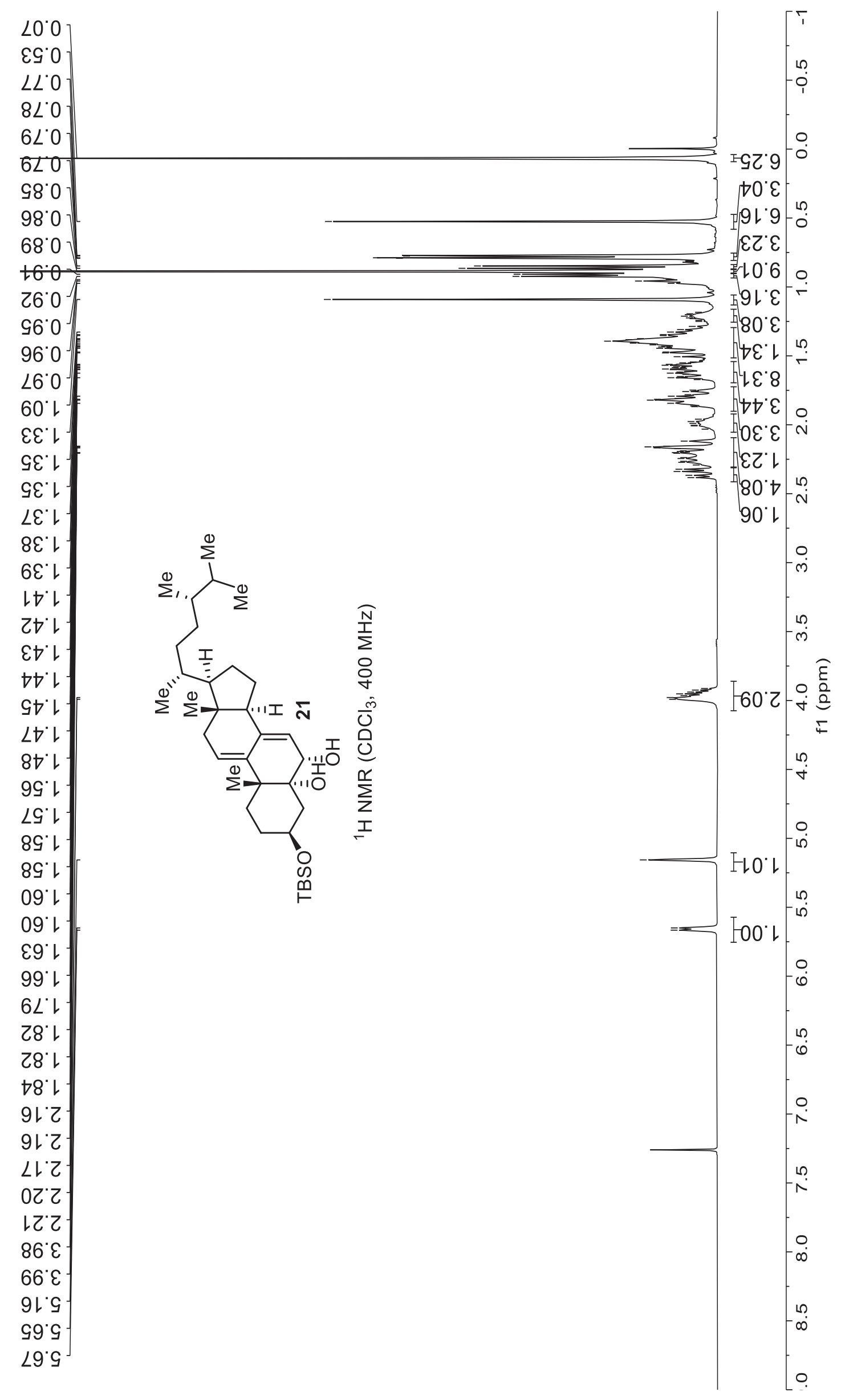




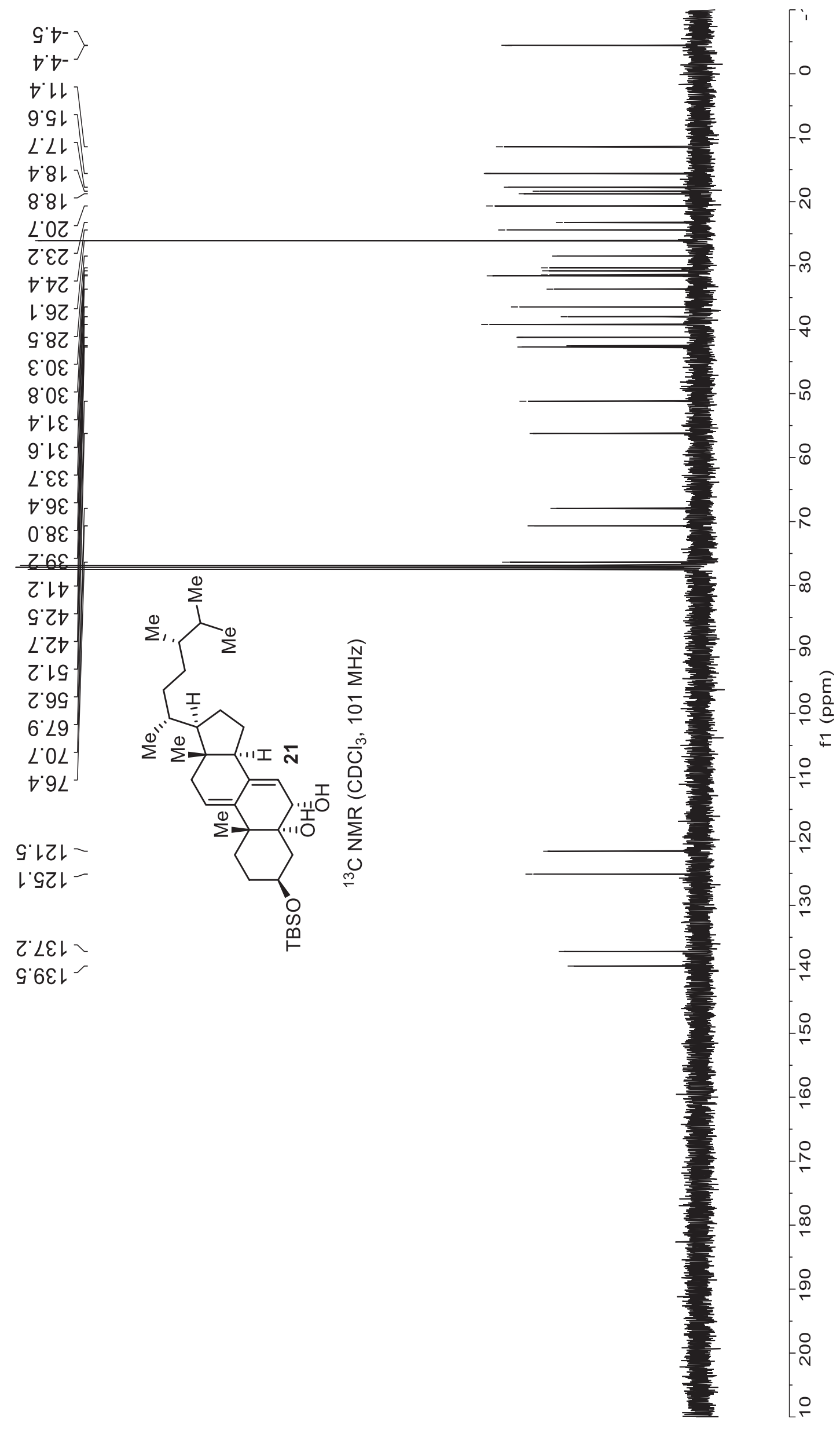




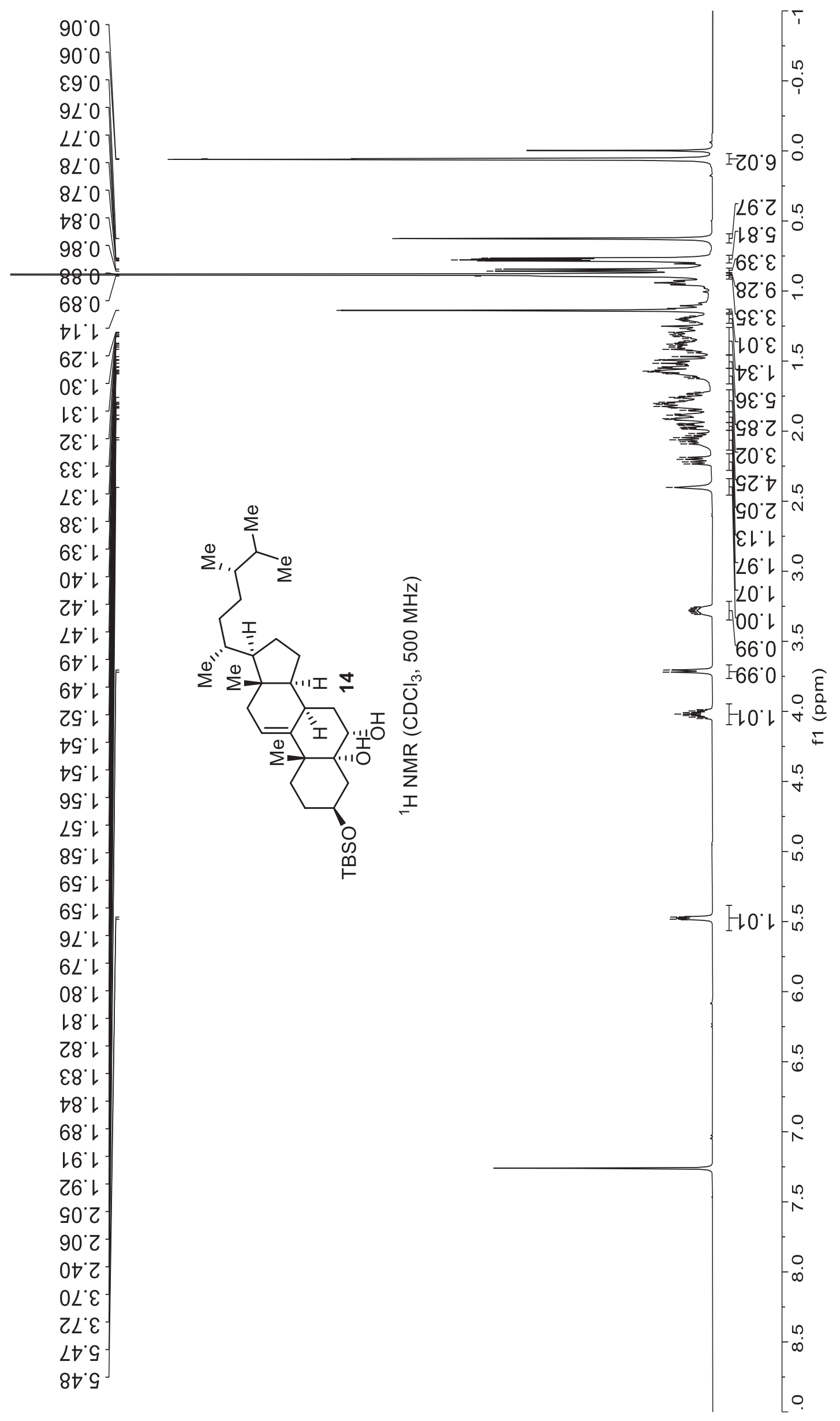




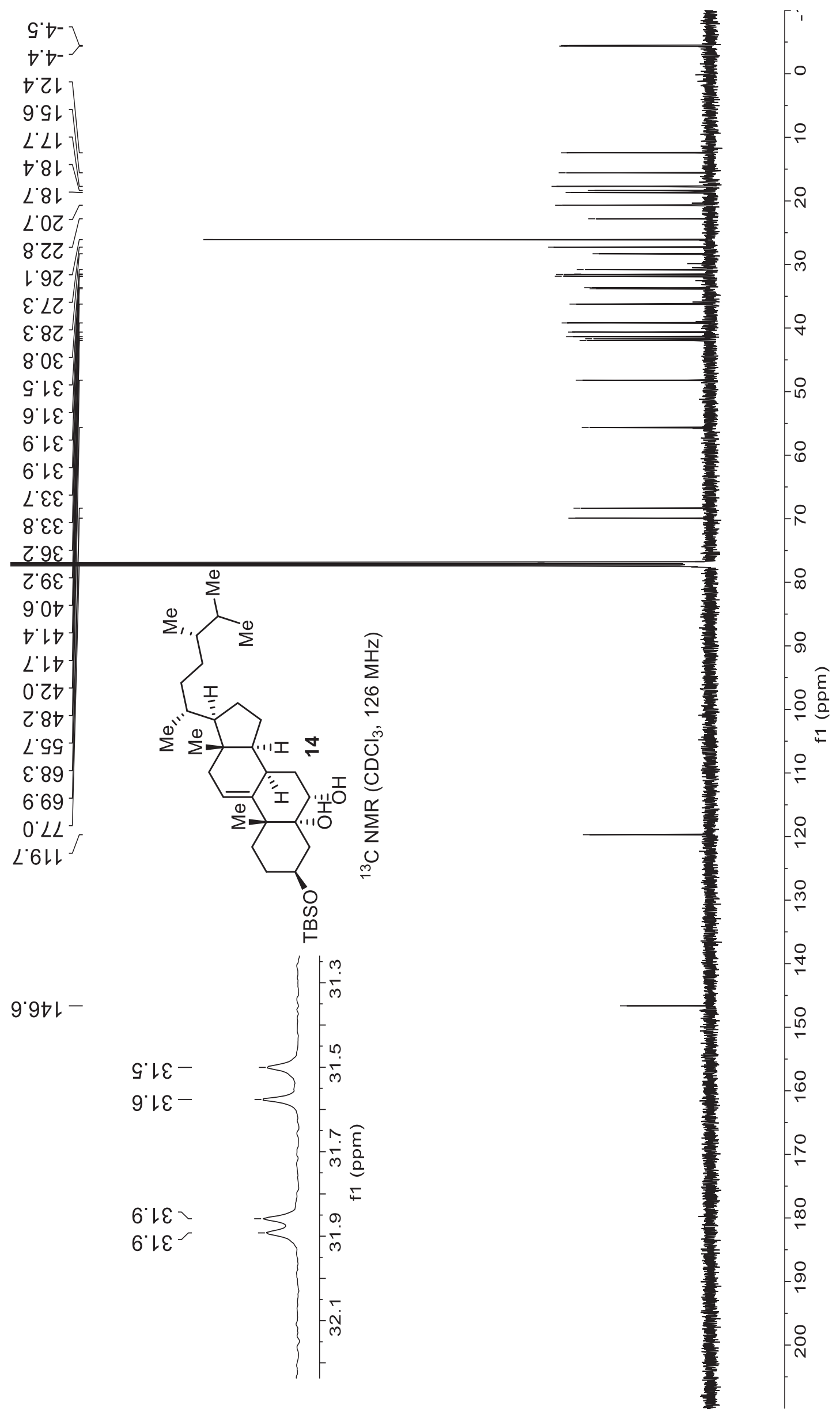




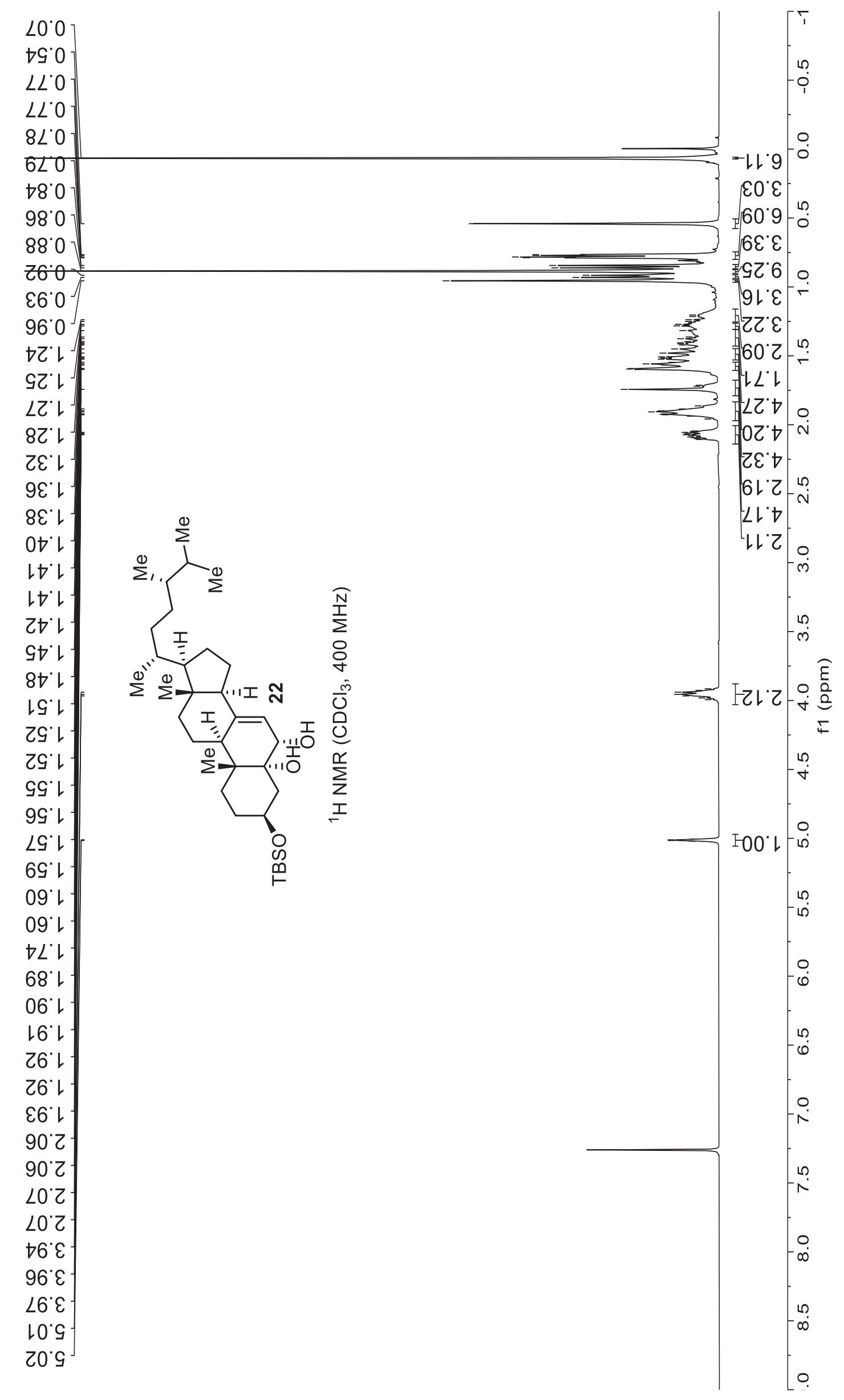




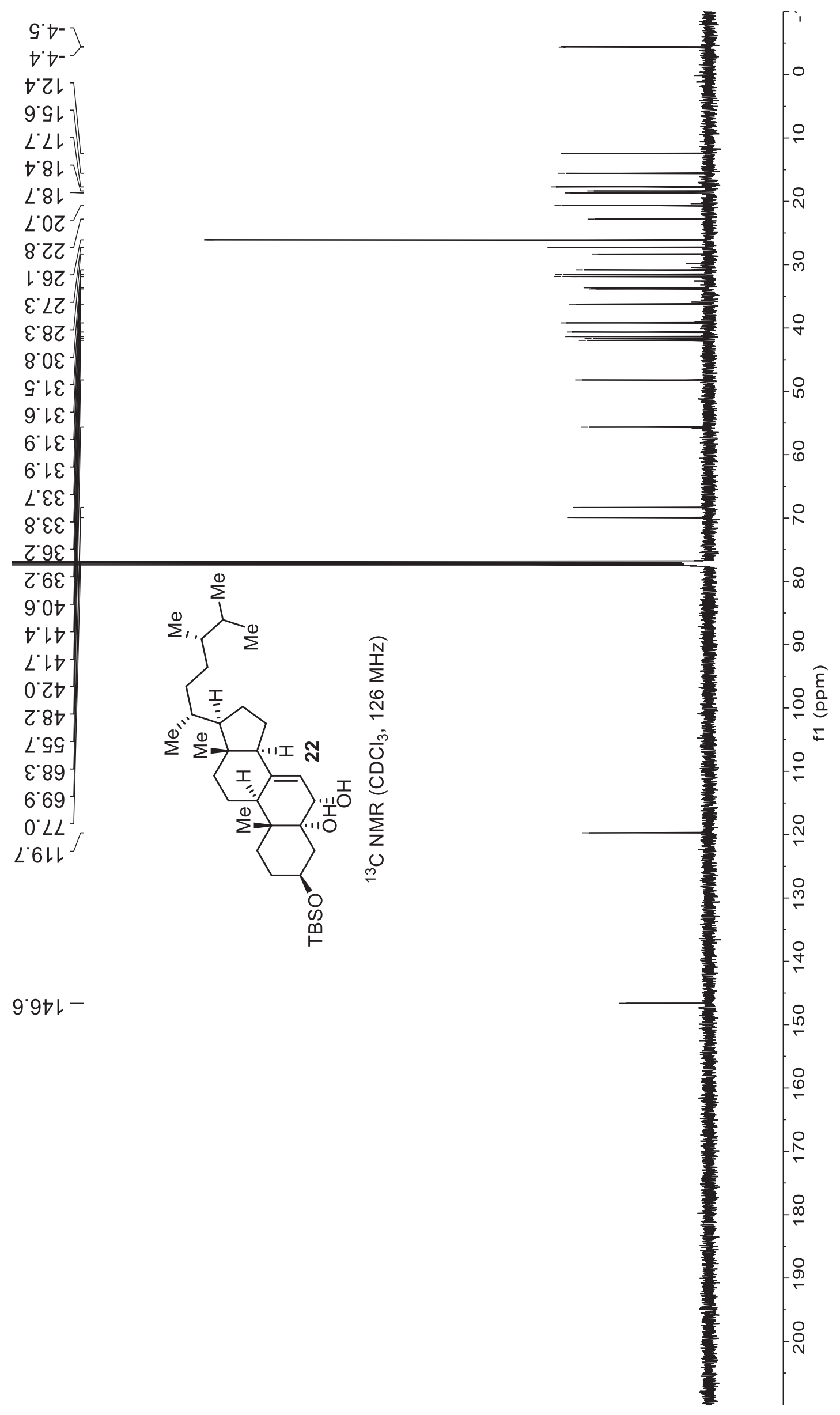




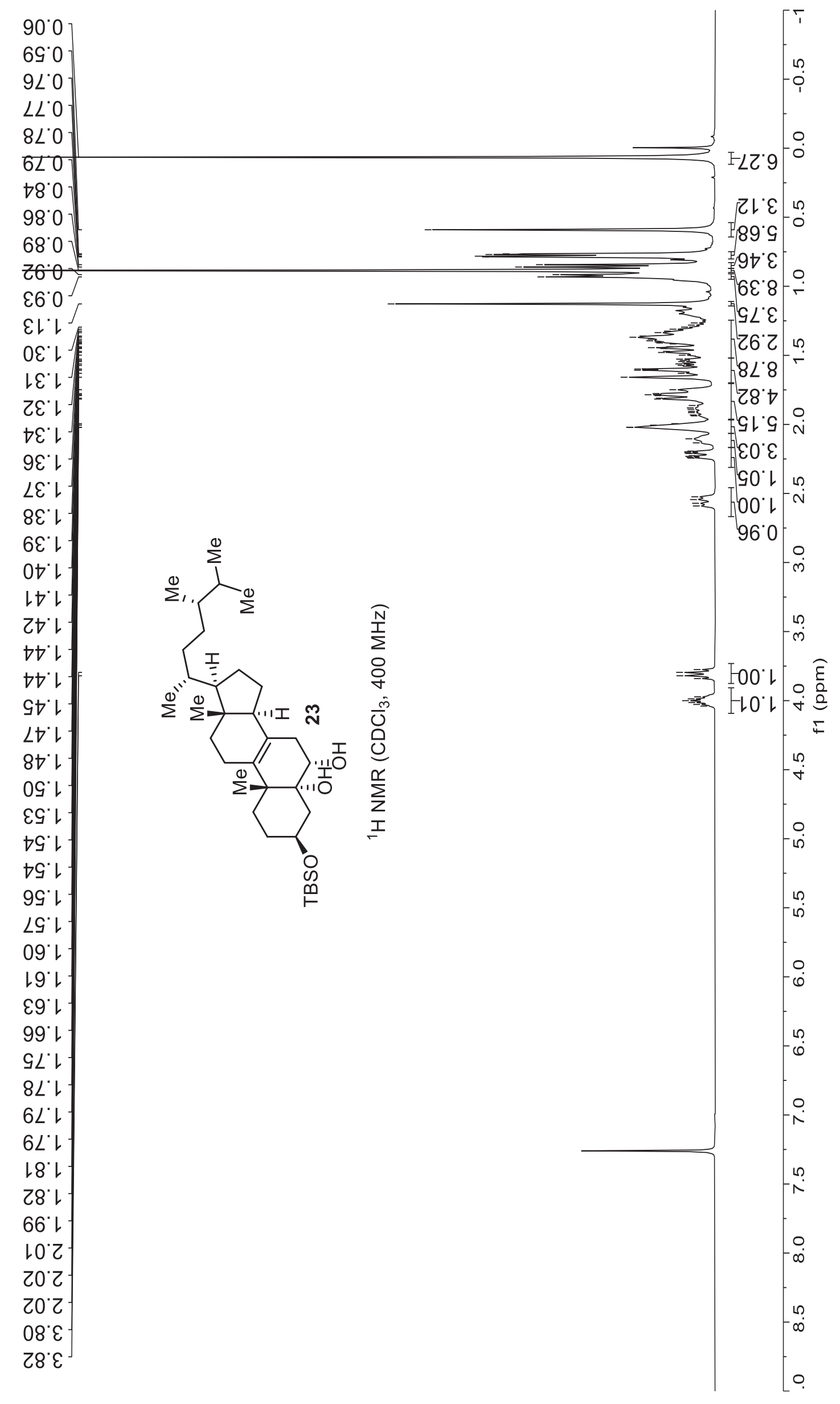




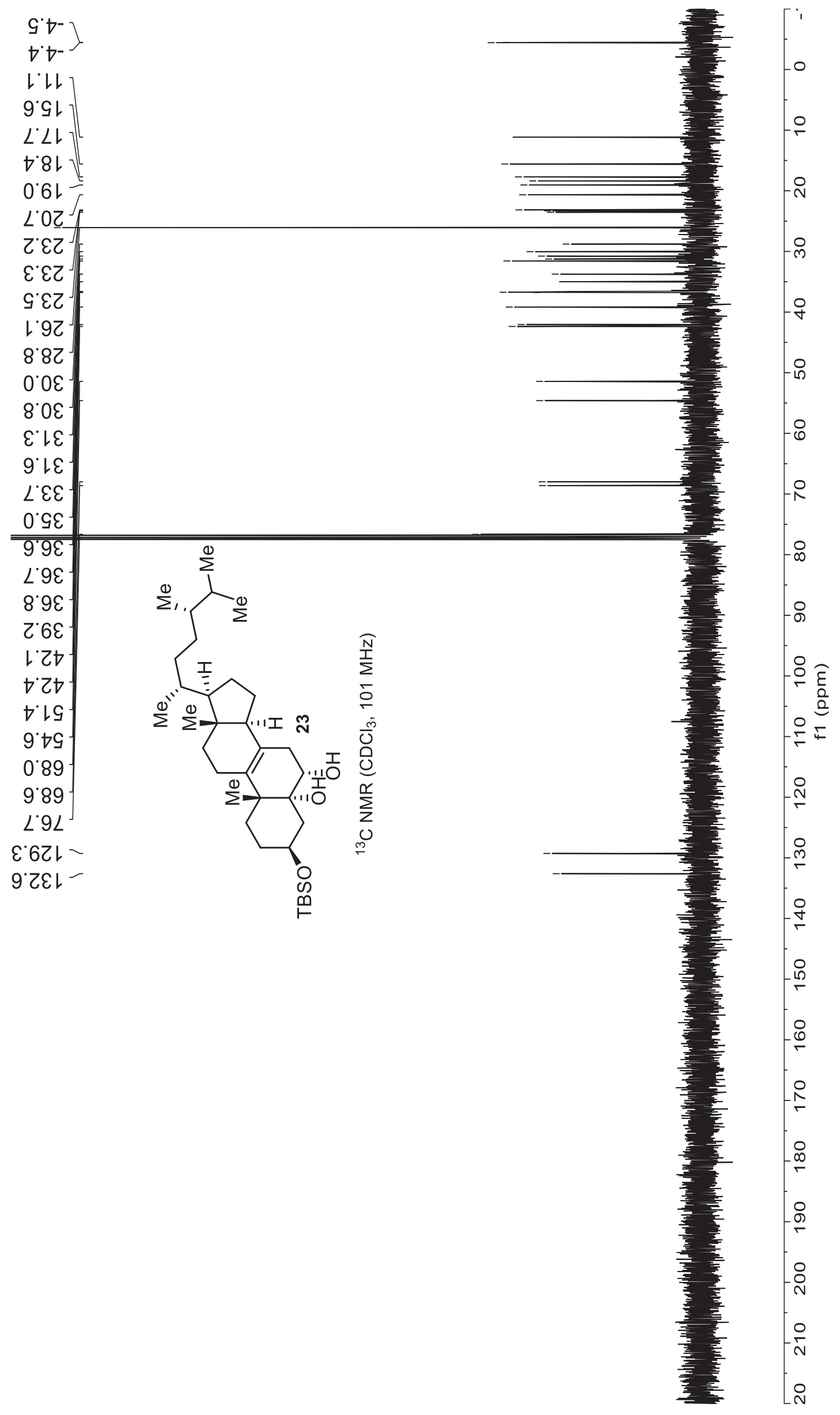




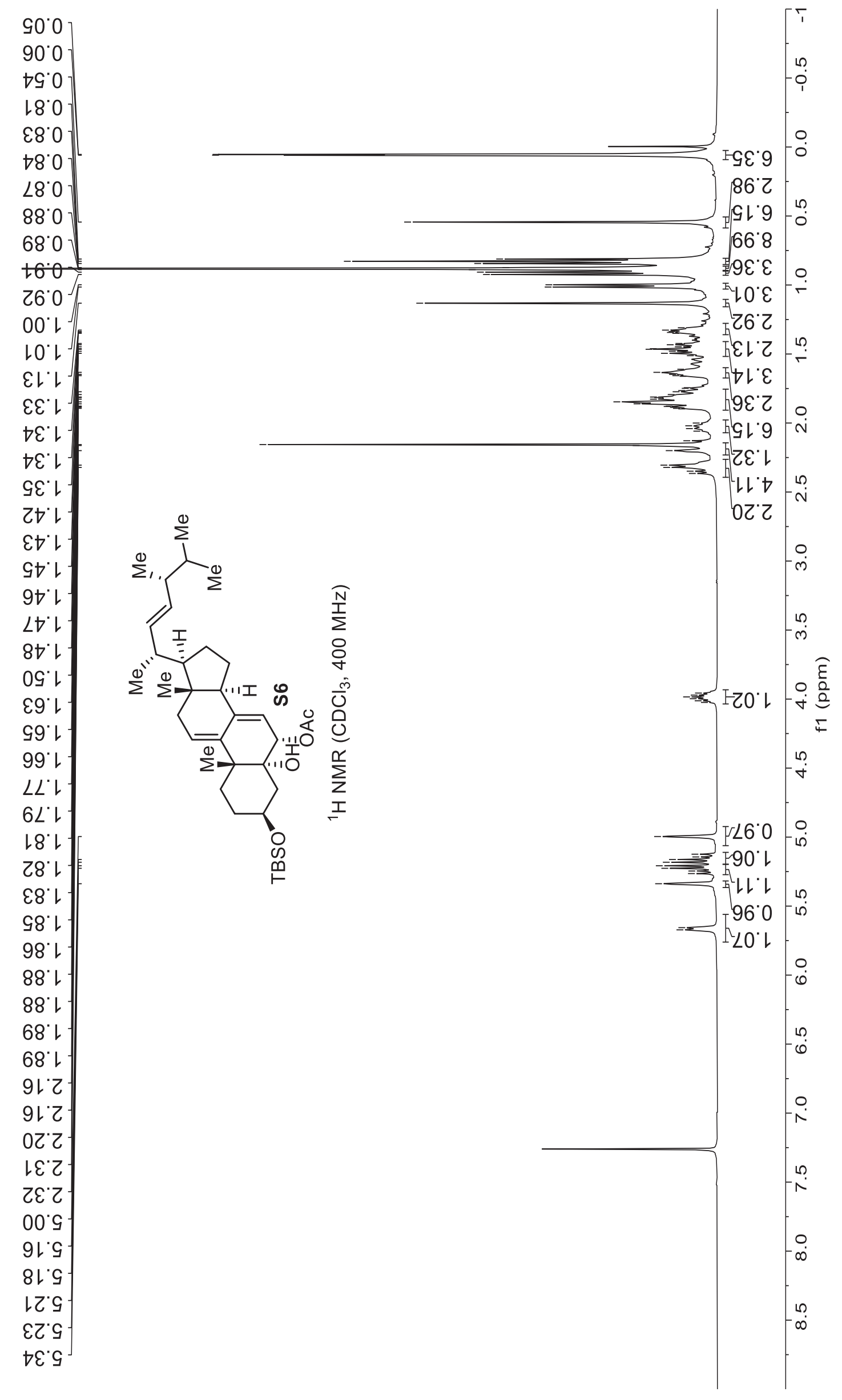




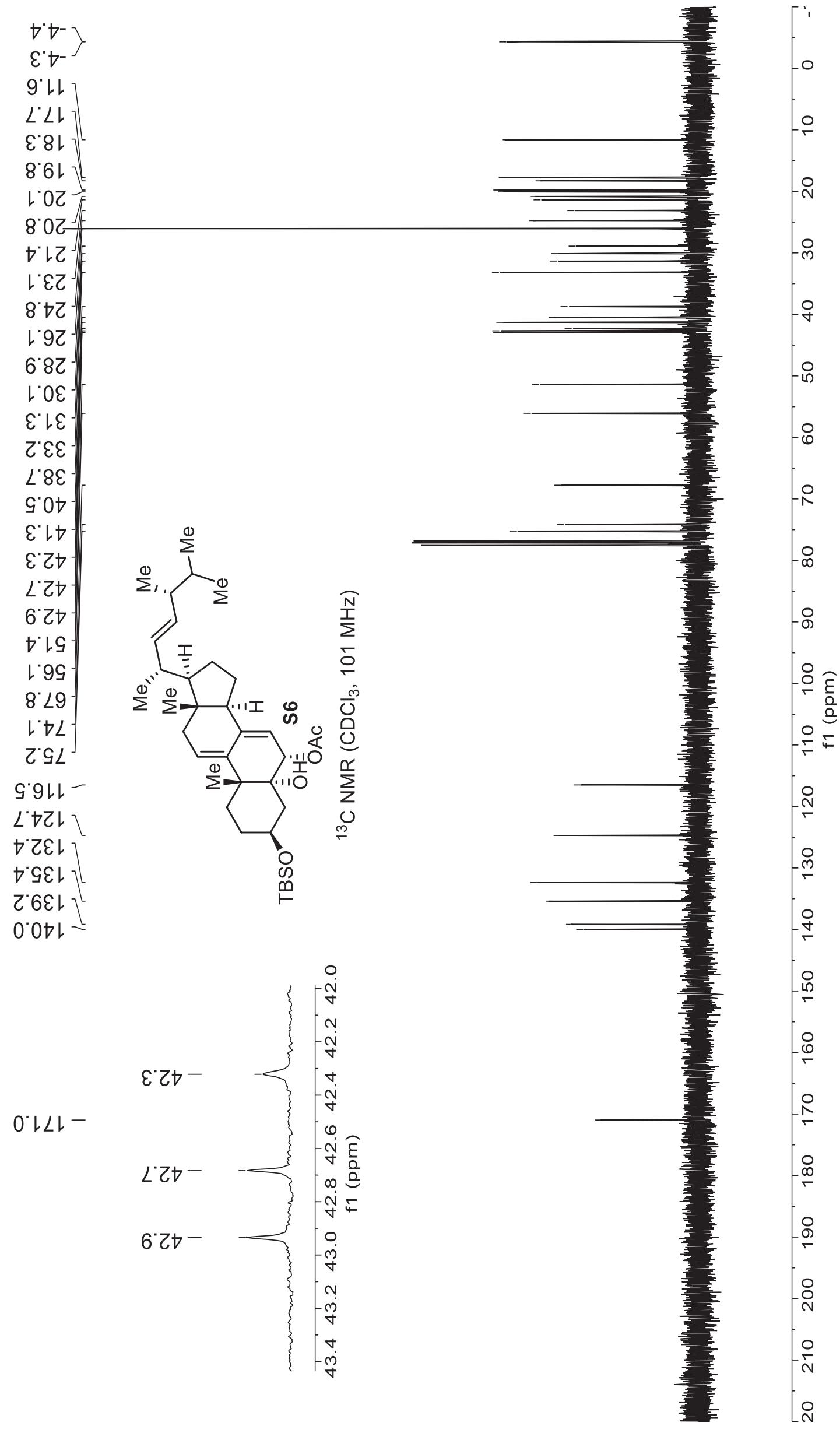




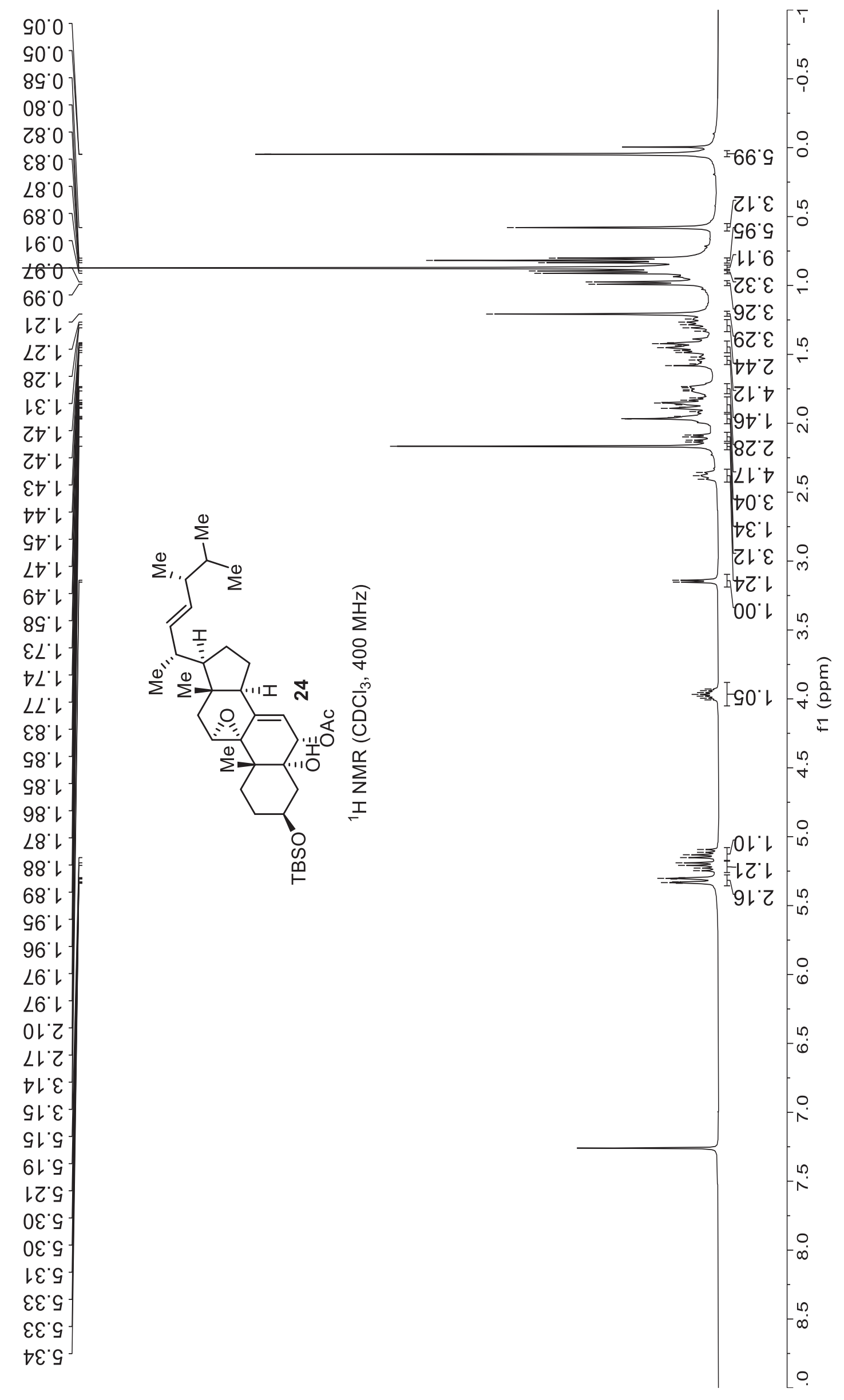




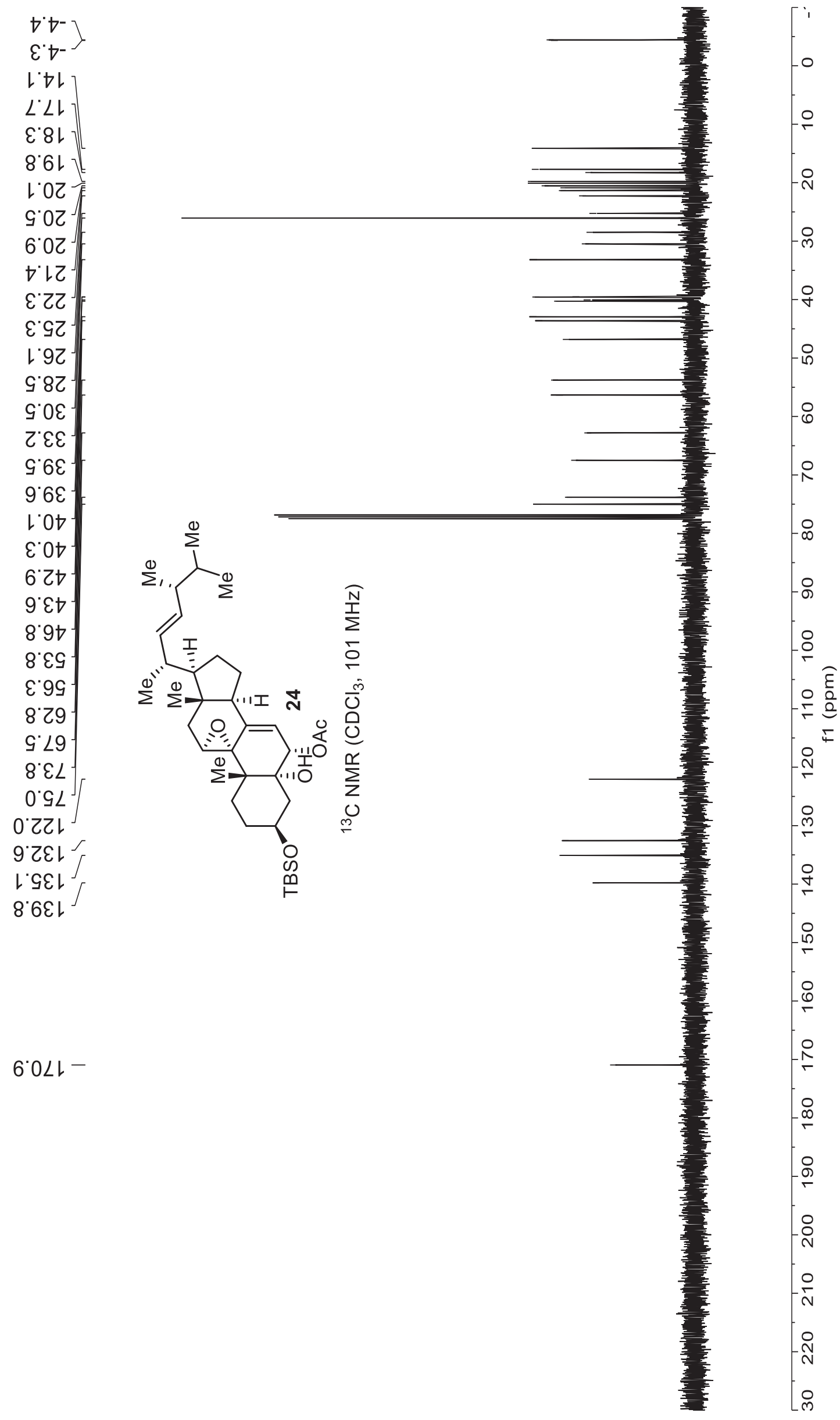




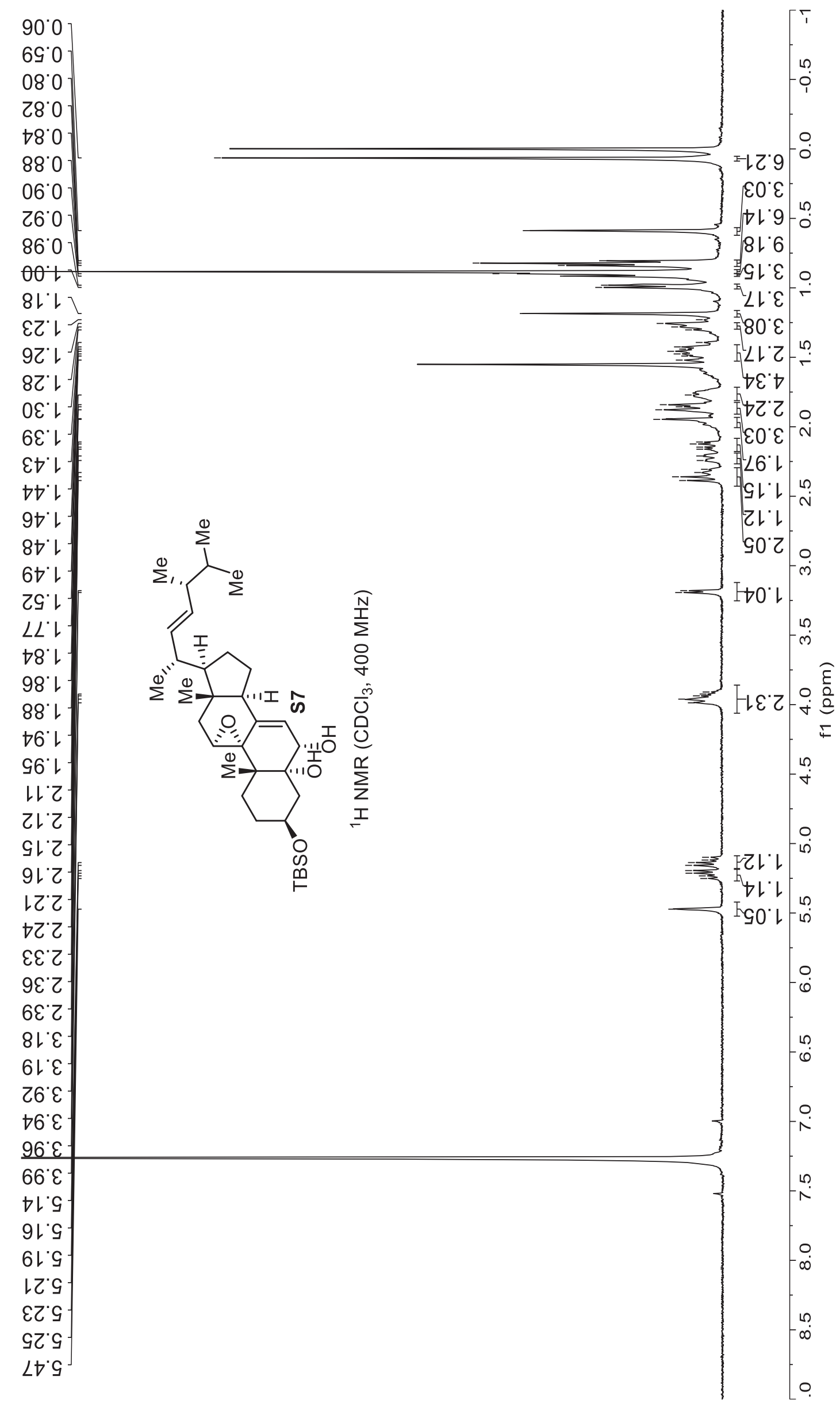




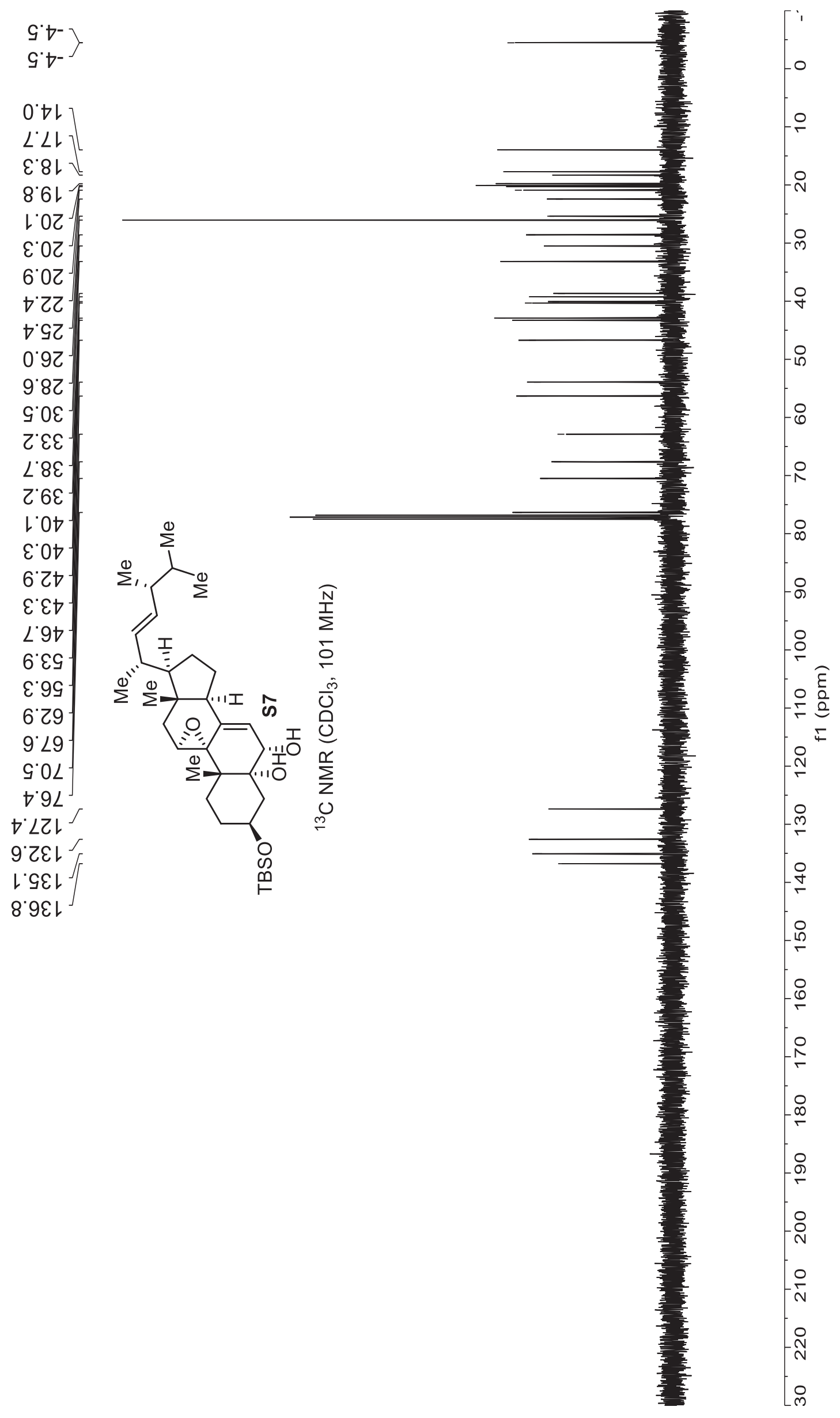


(mdd) if

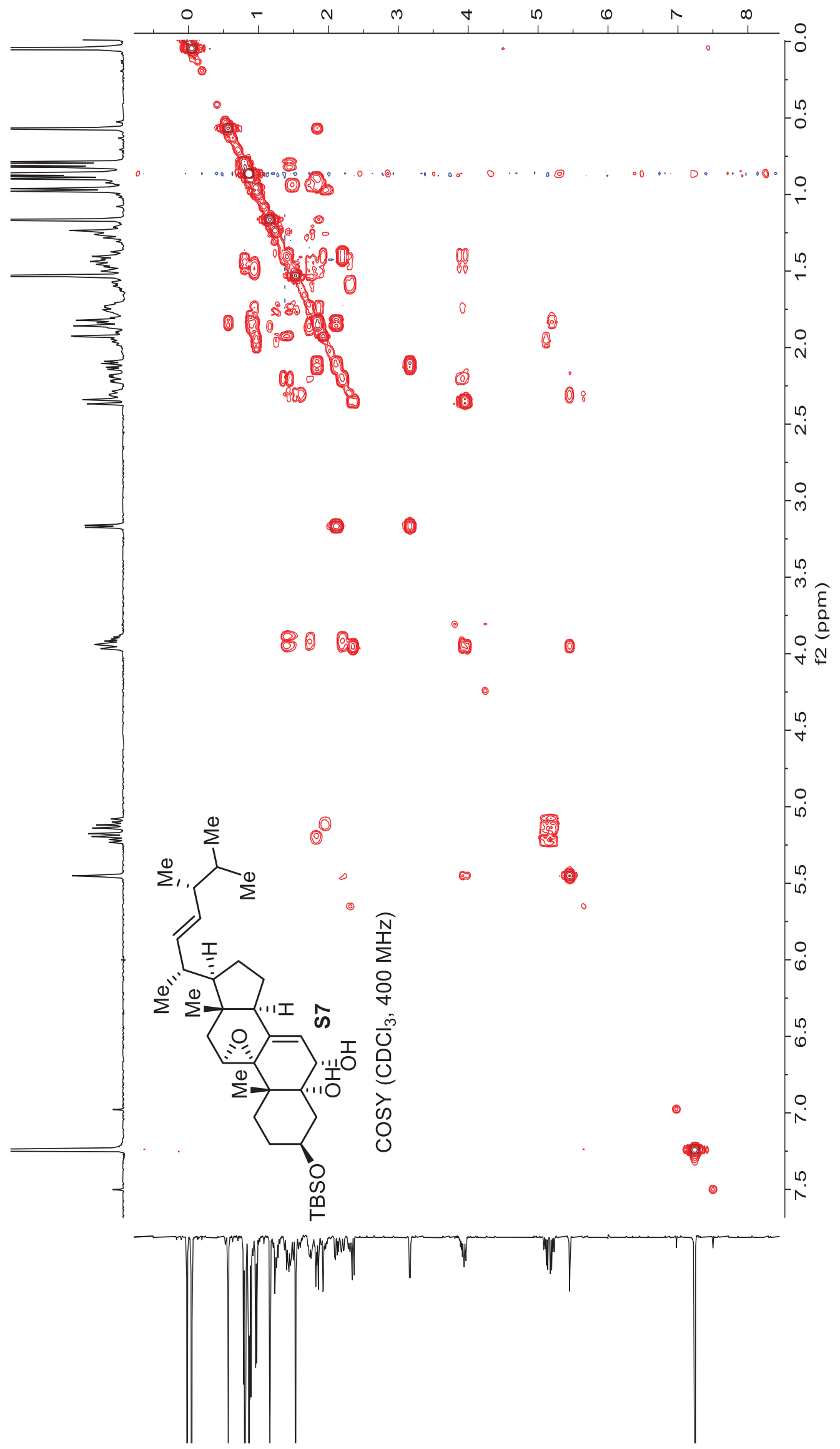




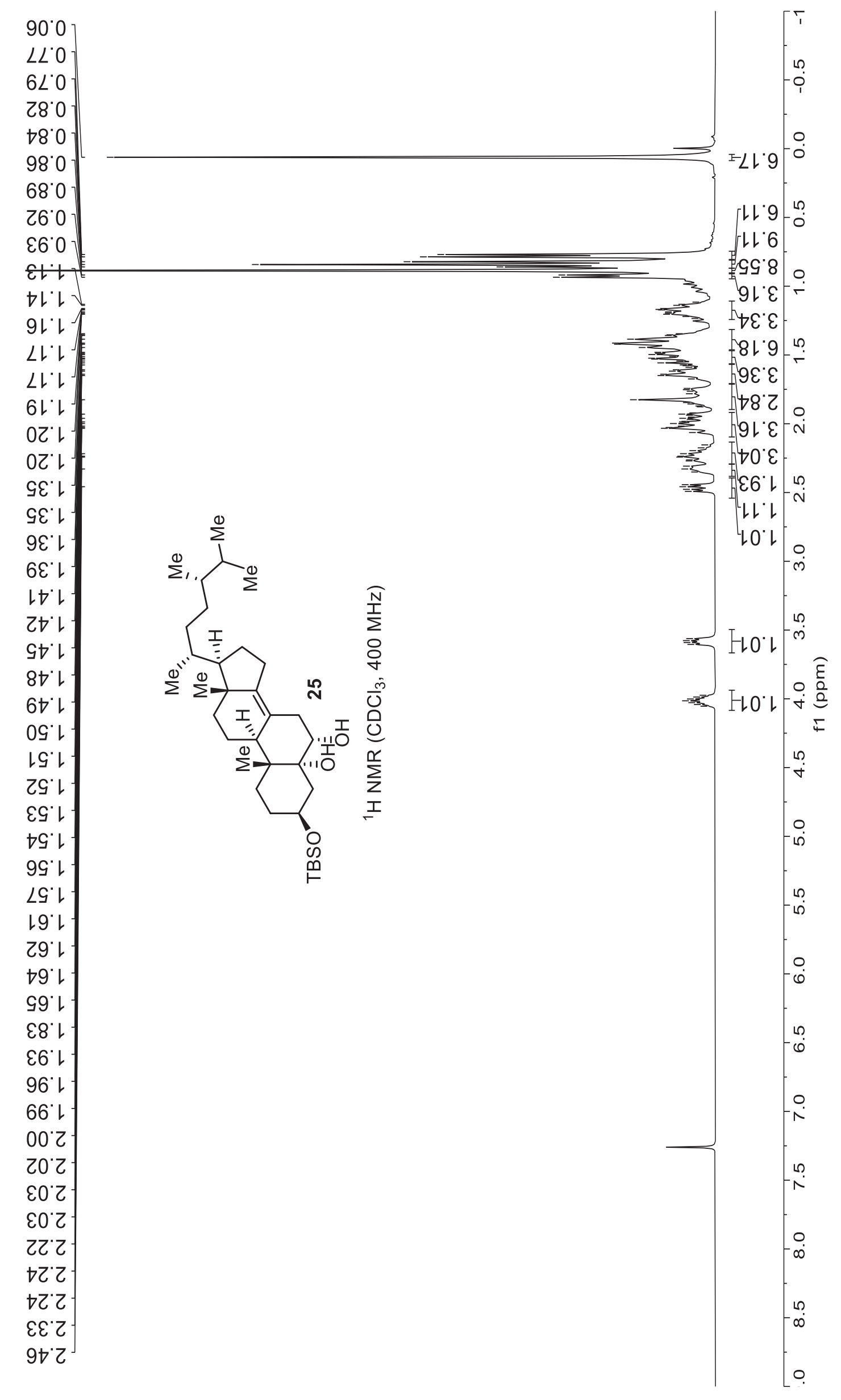




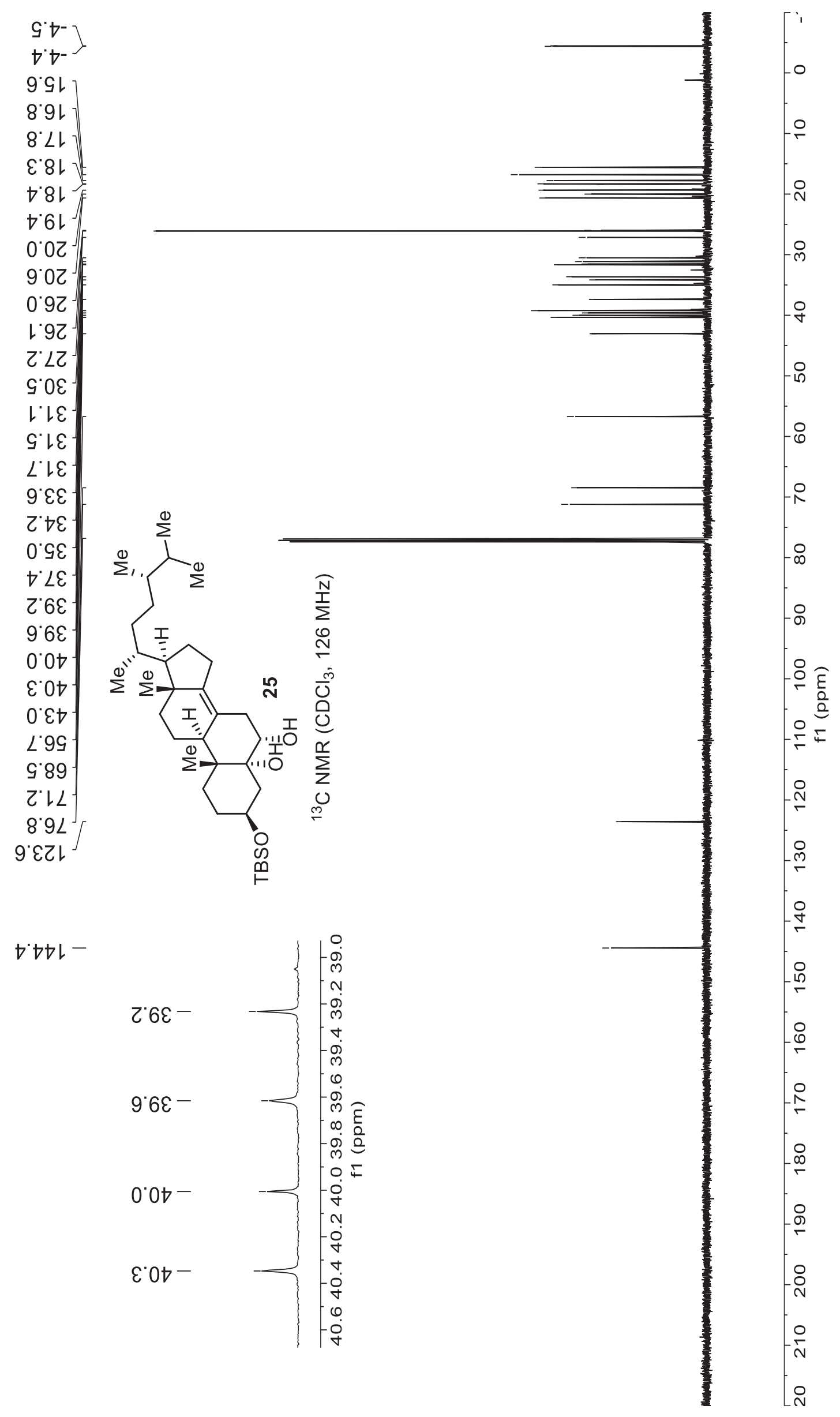




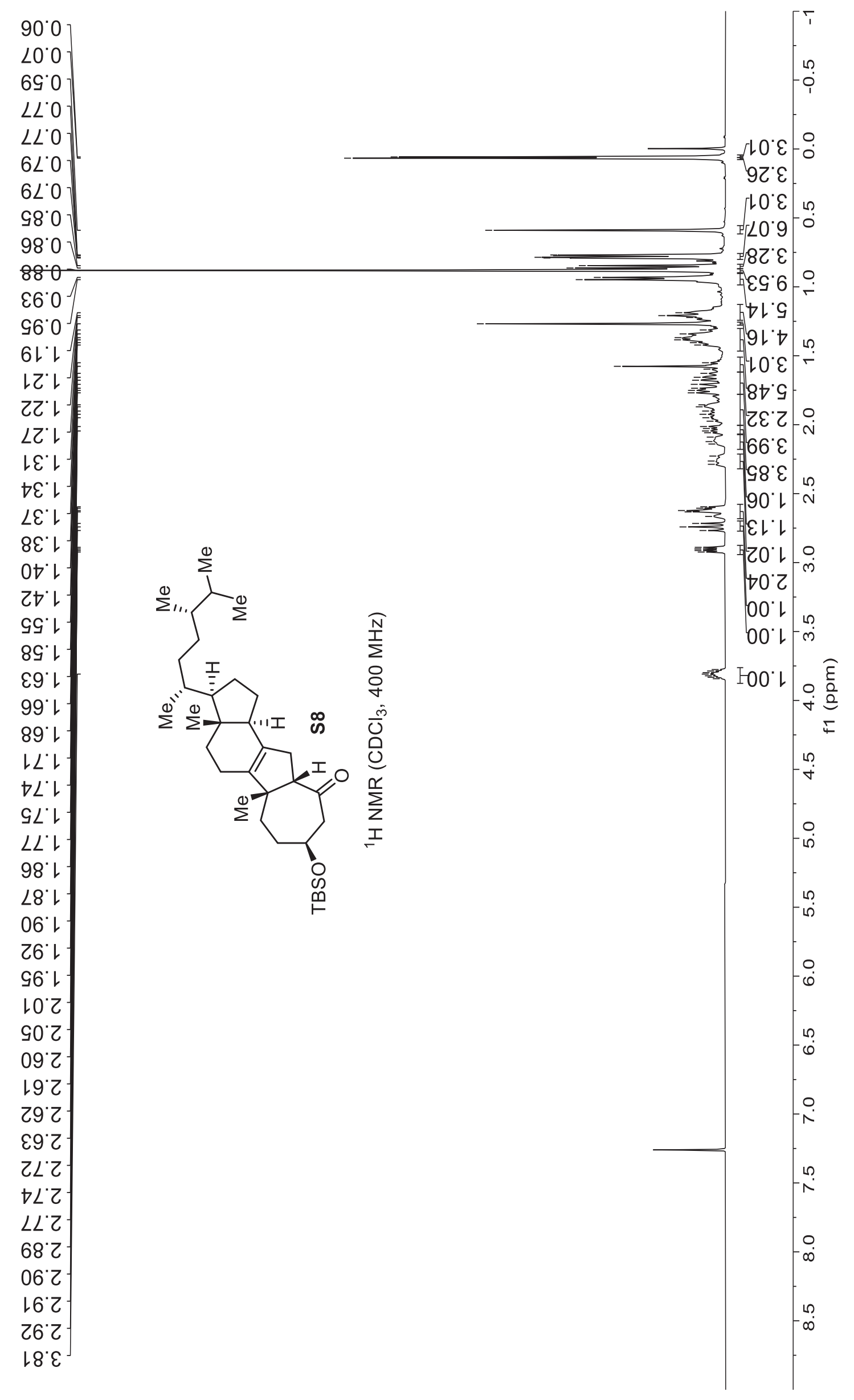




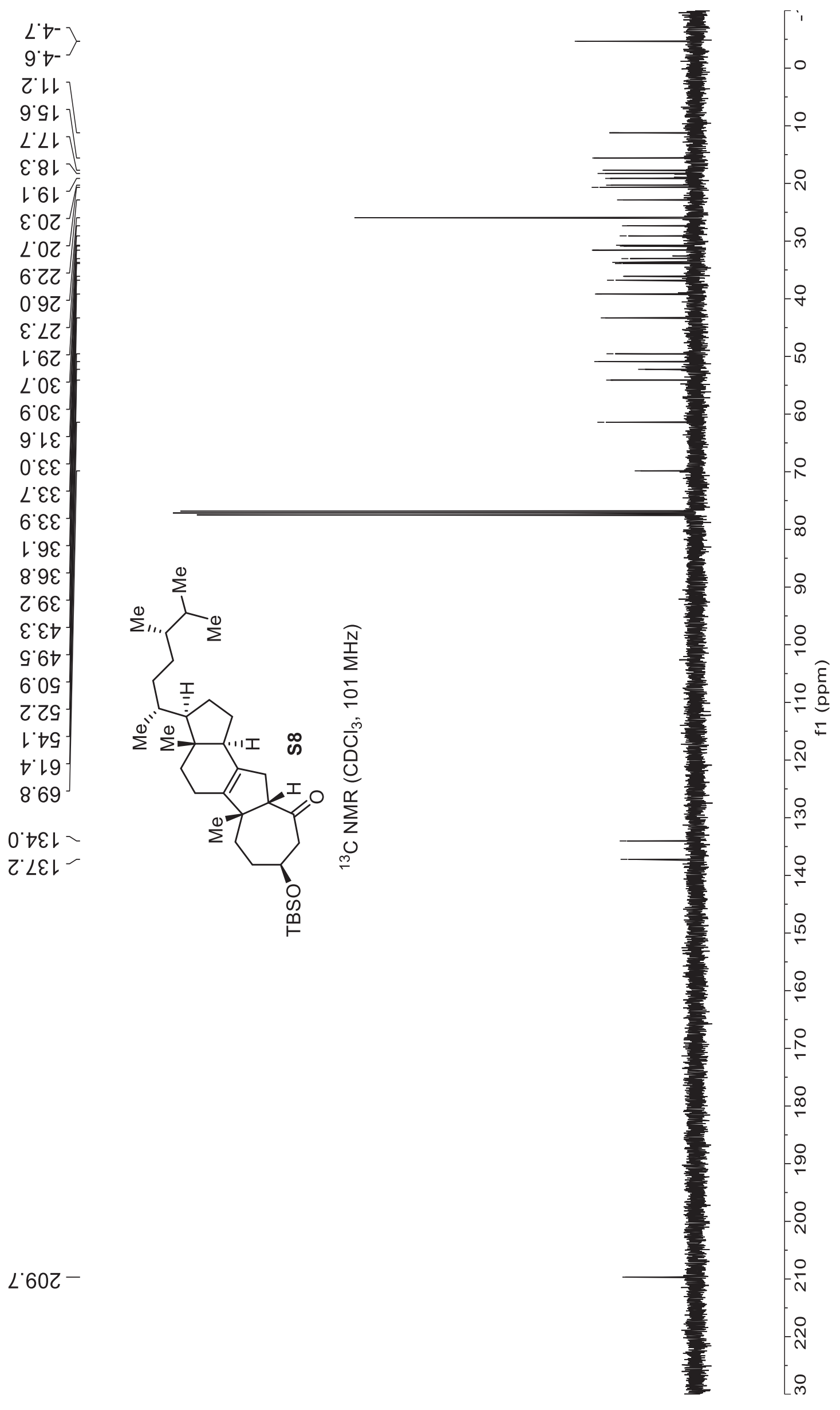




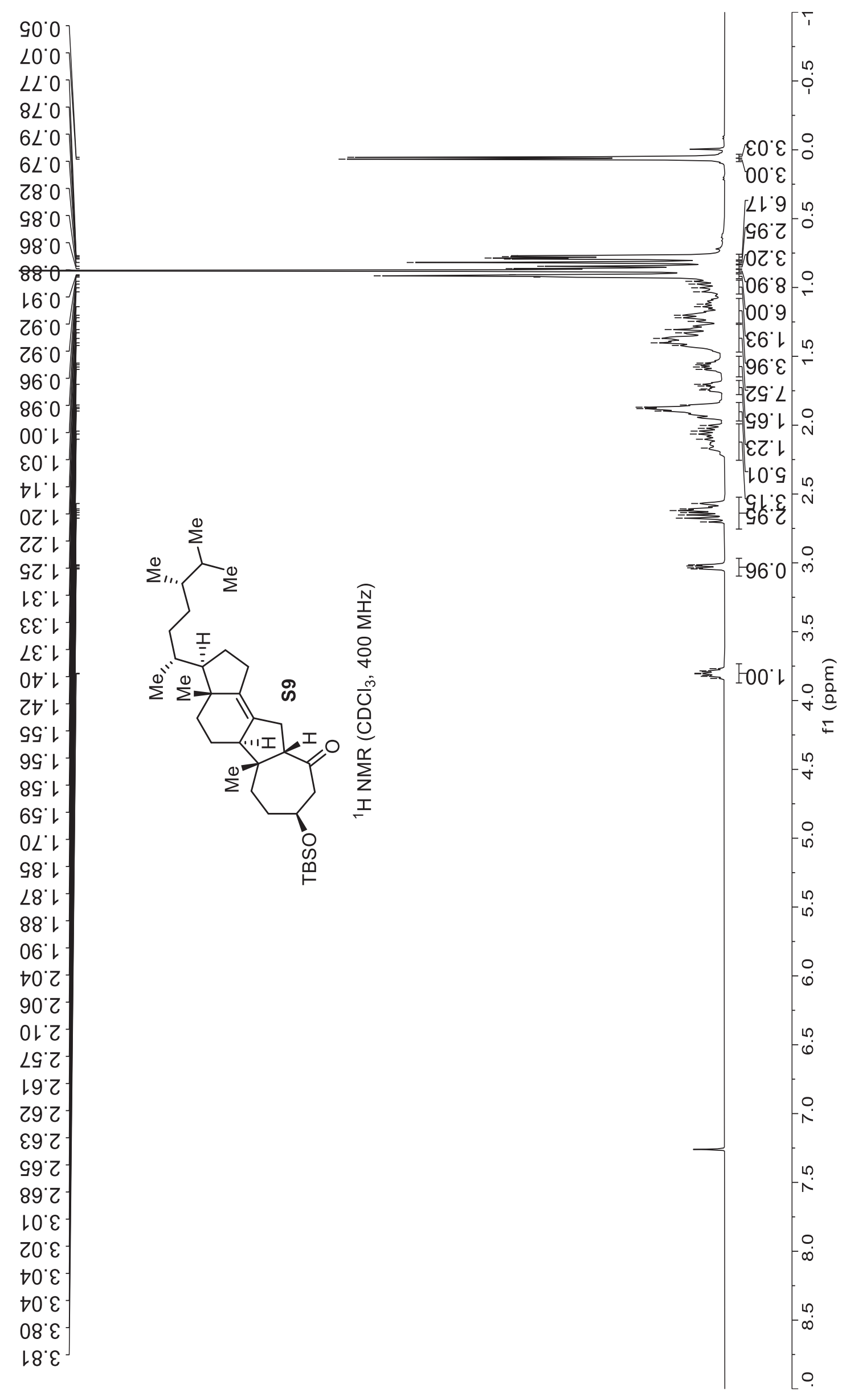




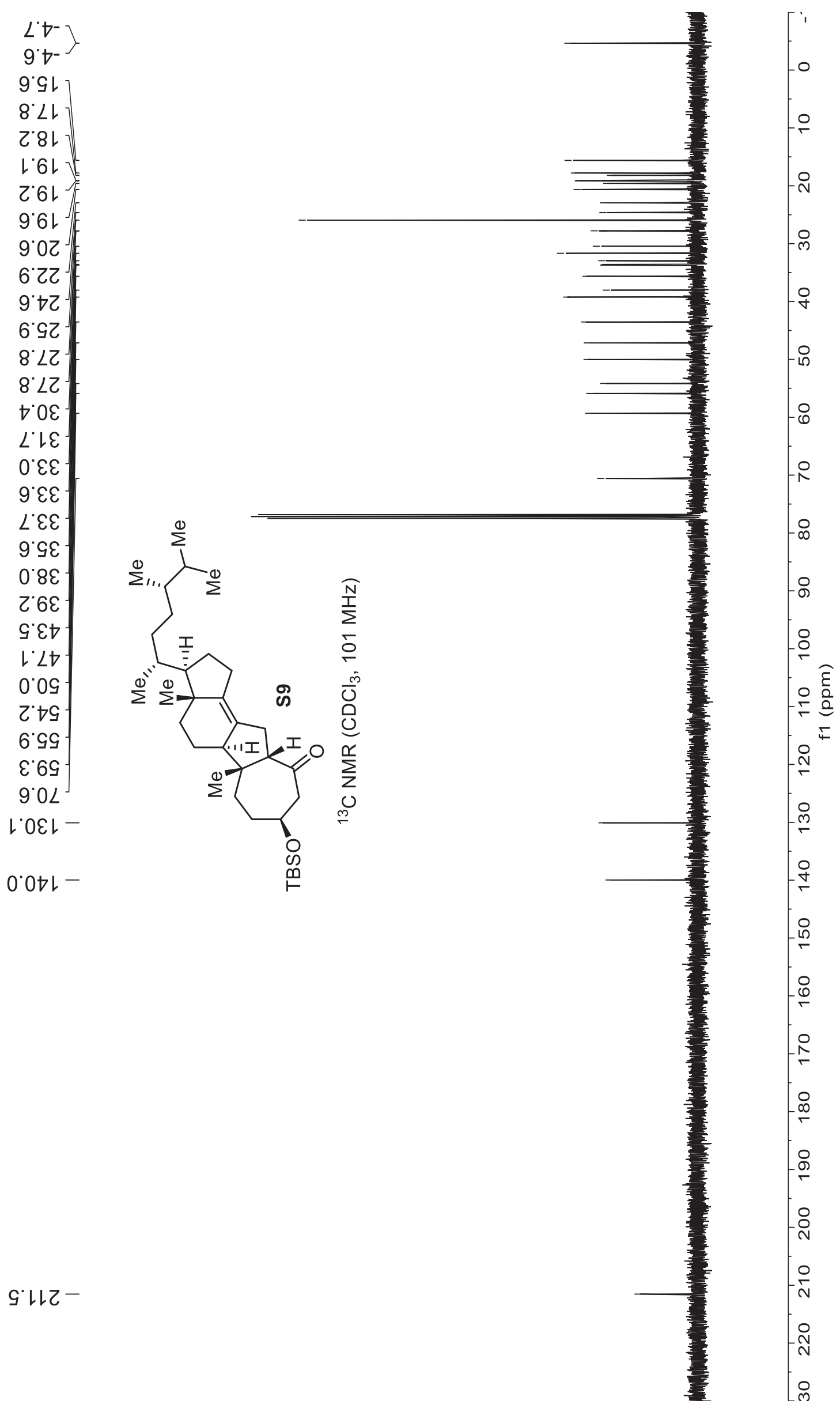




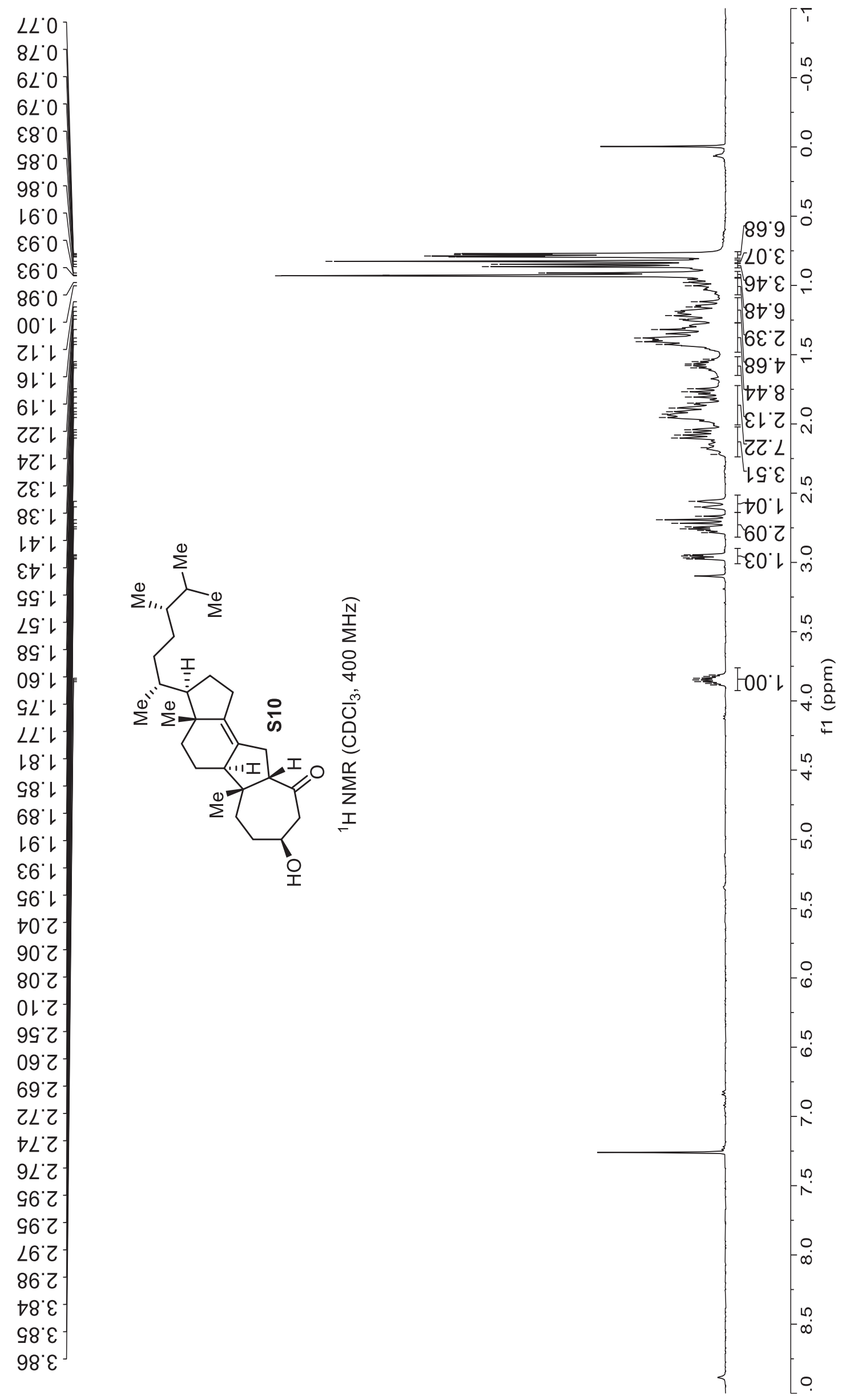




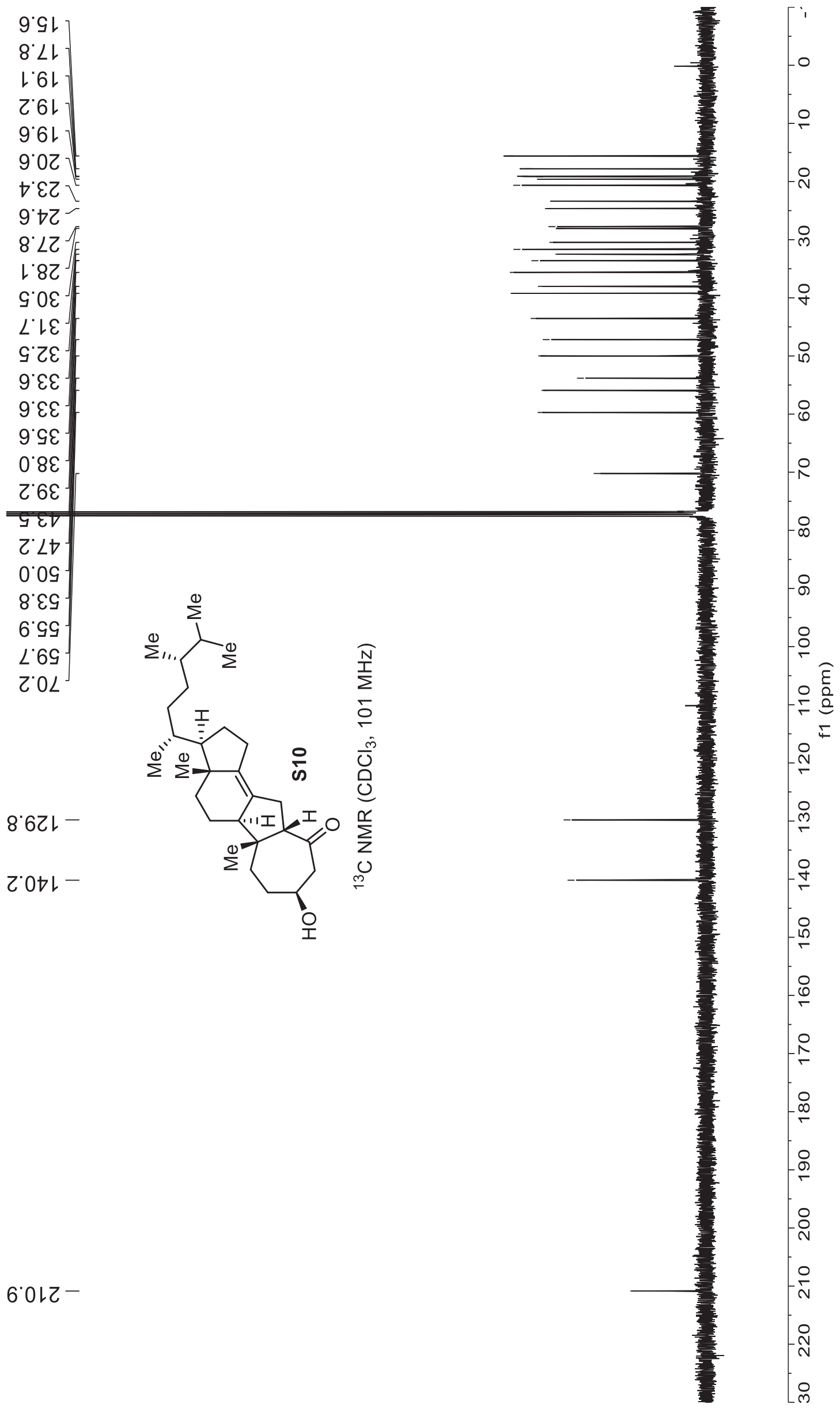




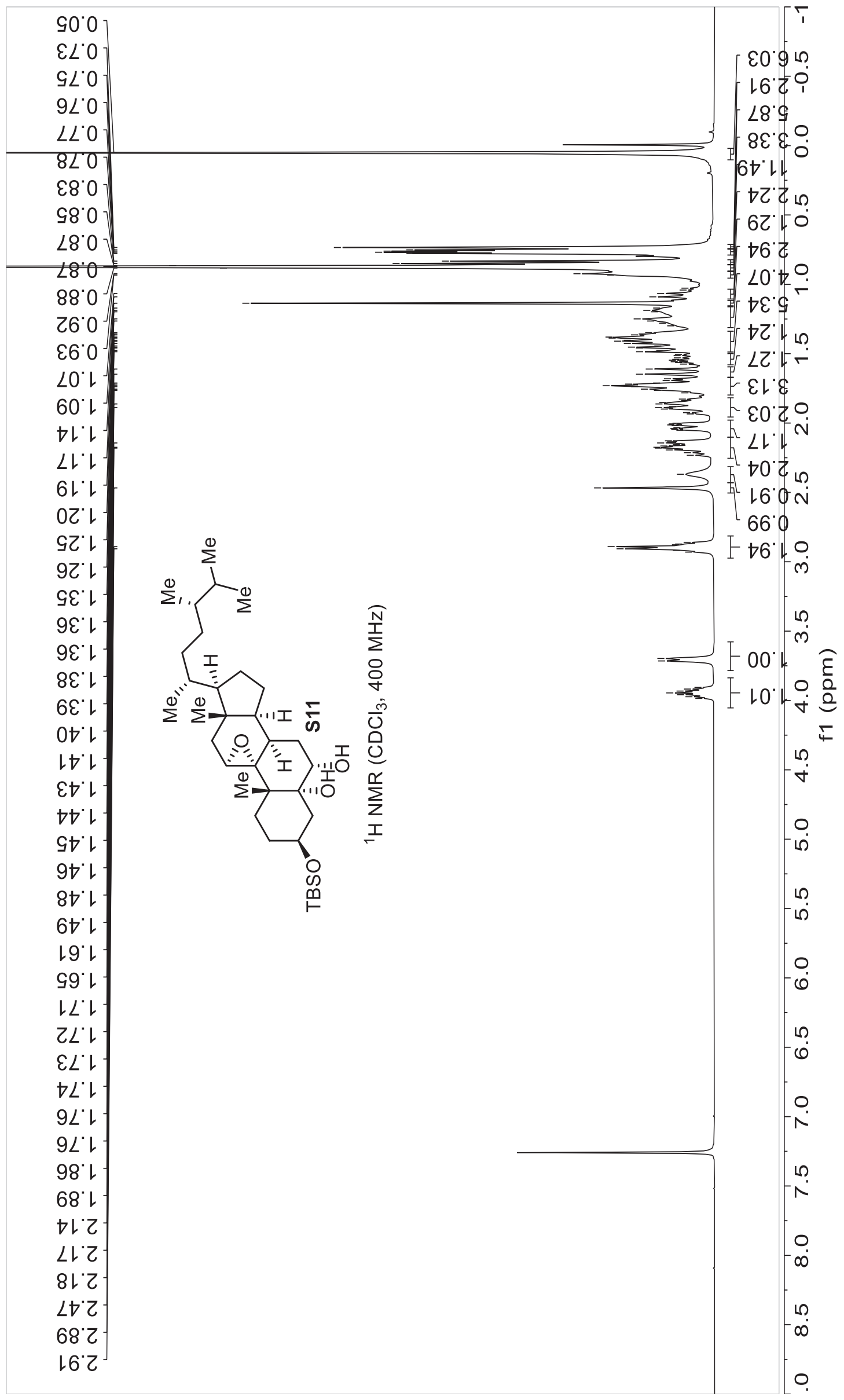




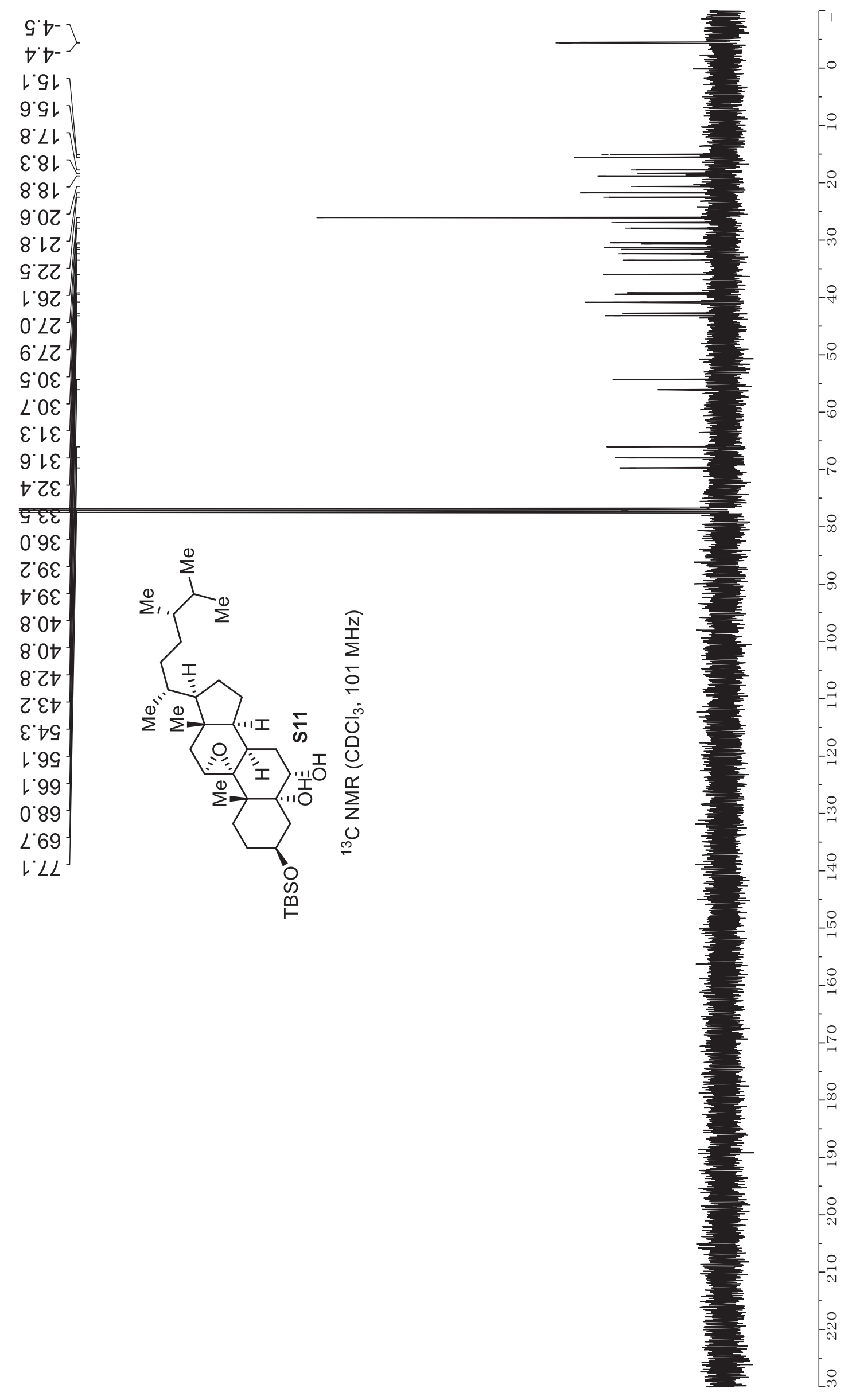




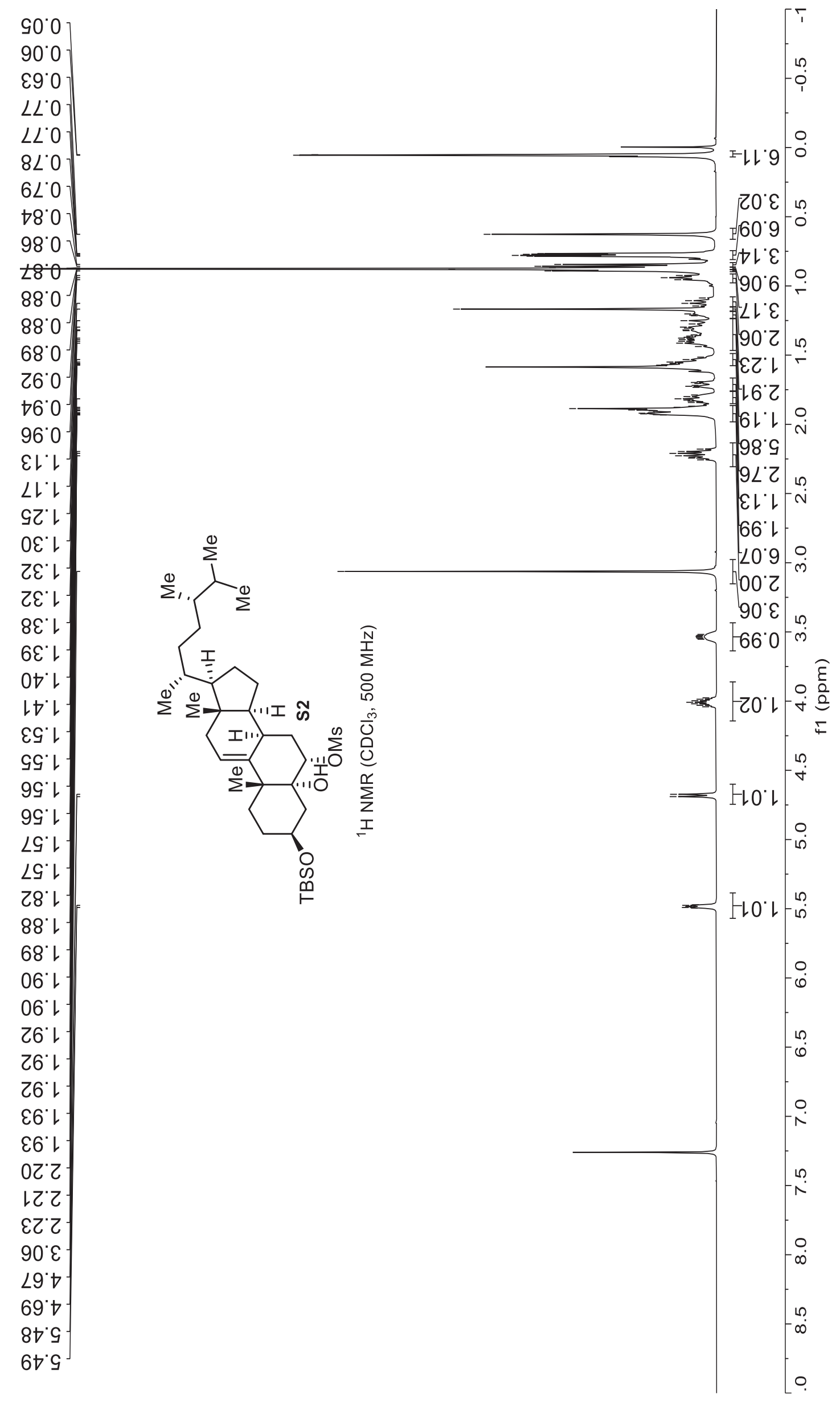




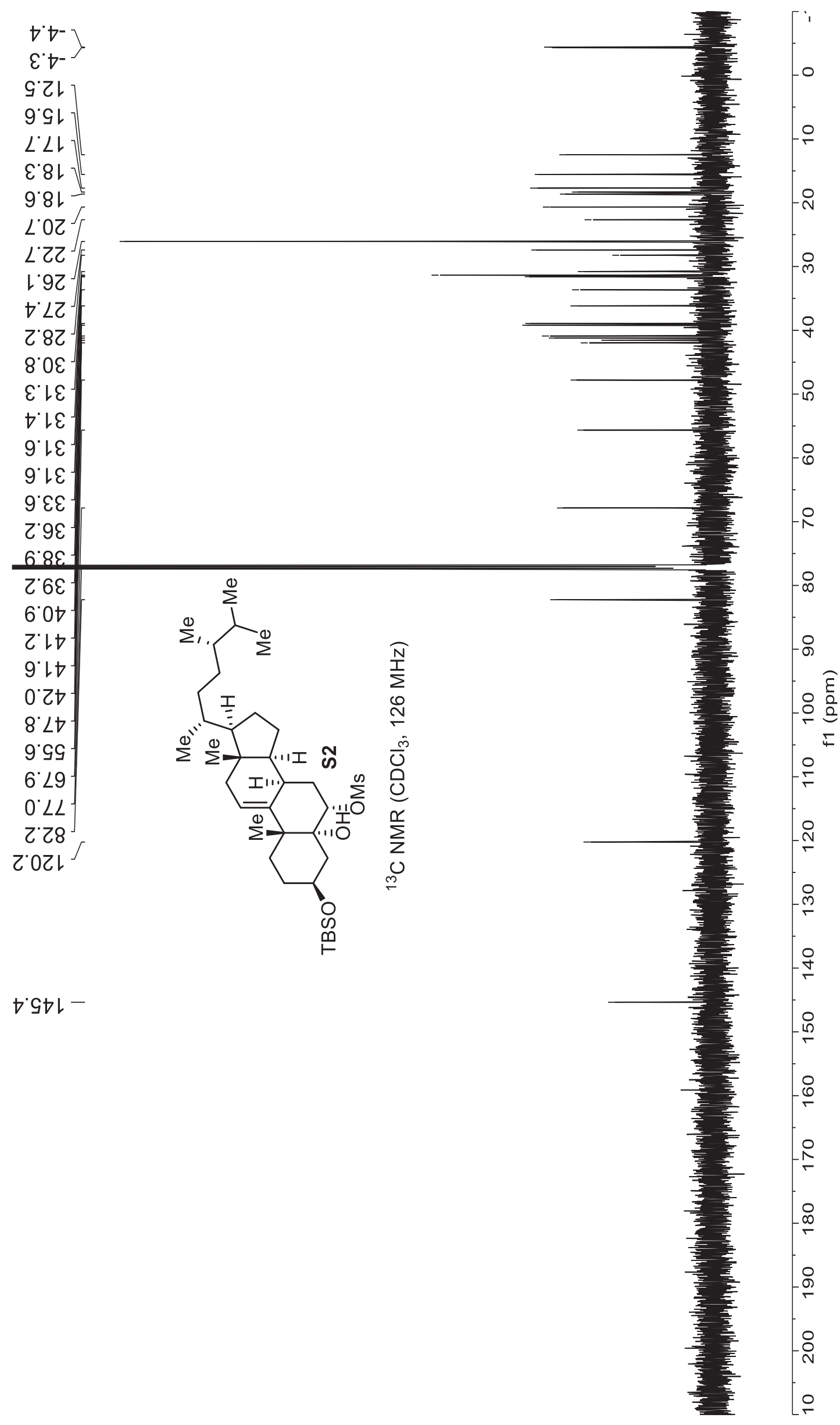




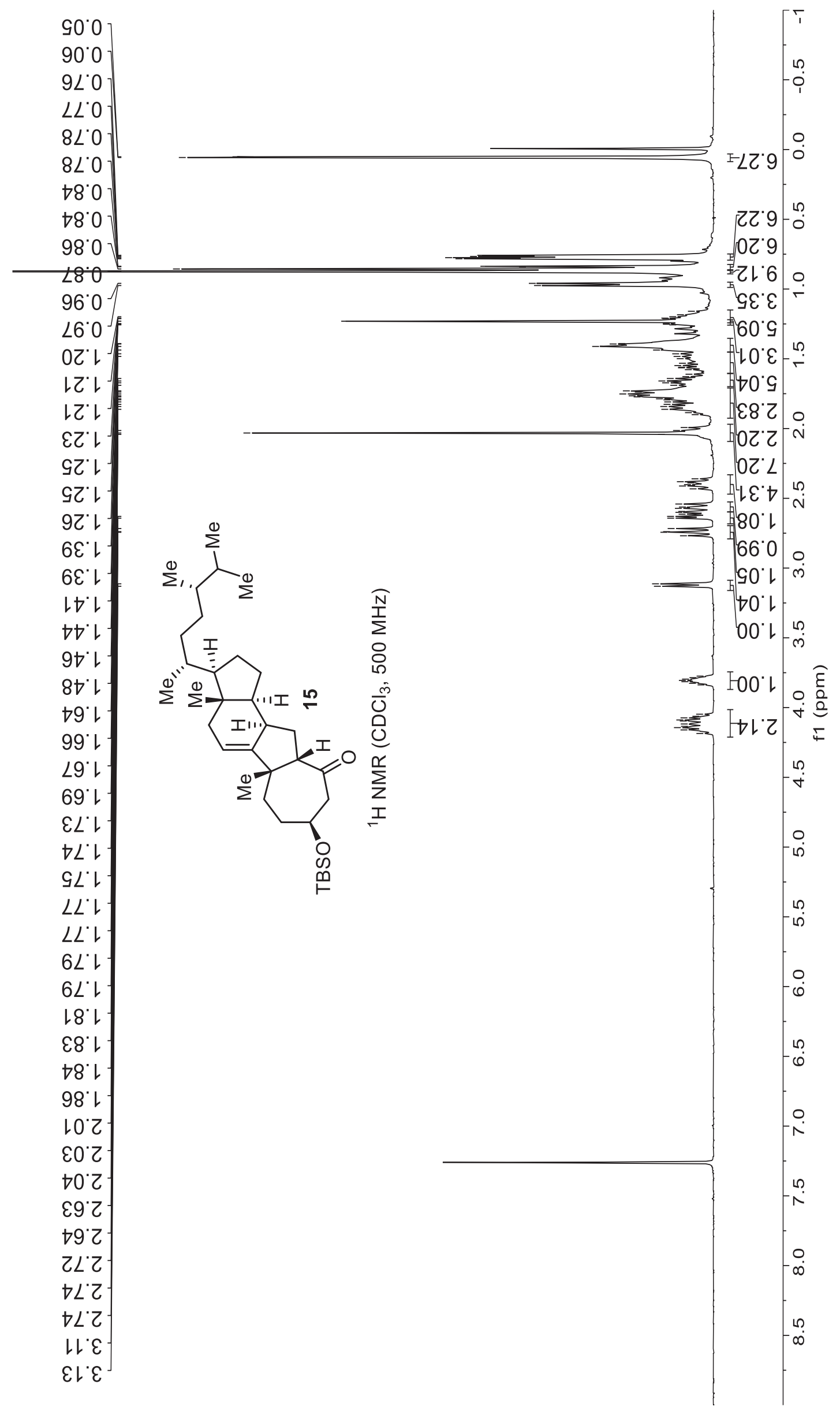




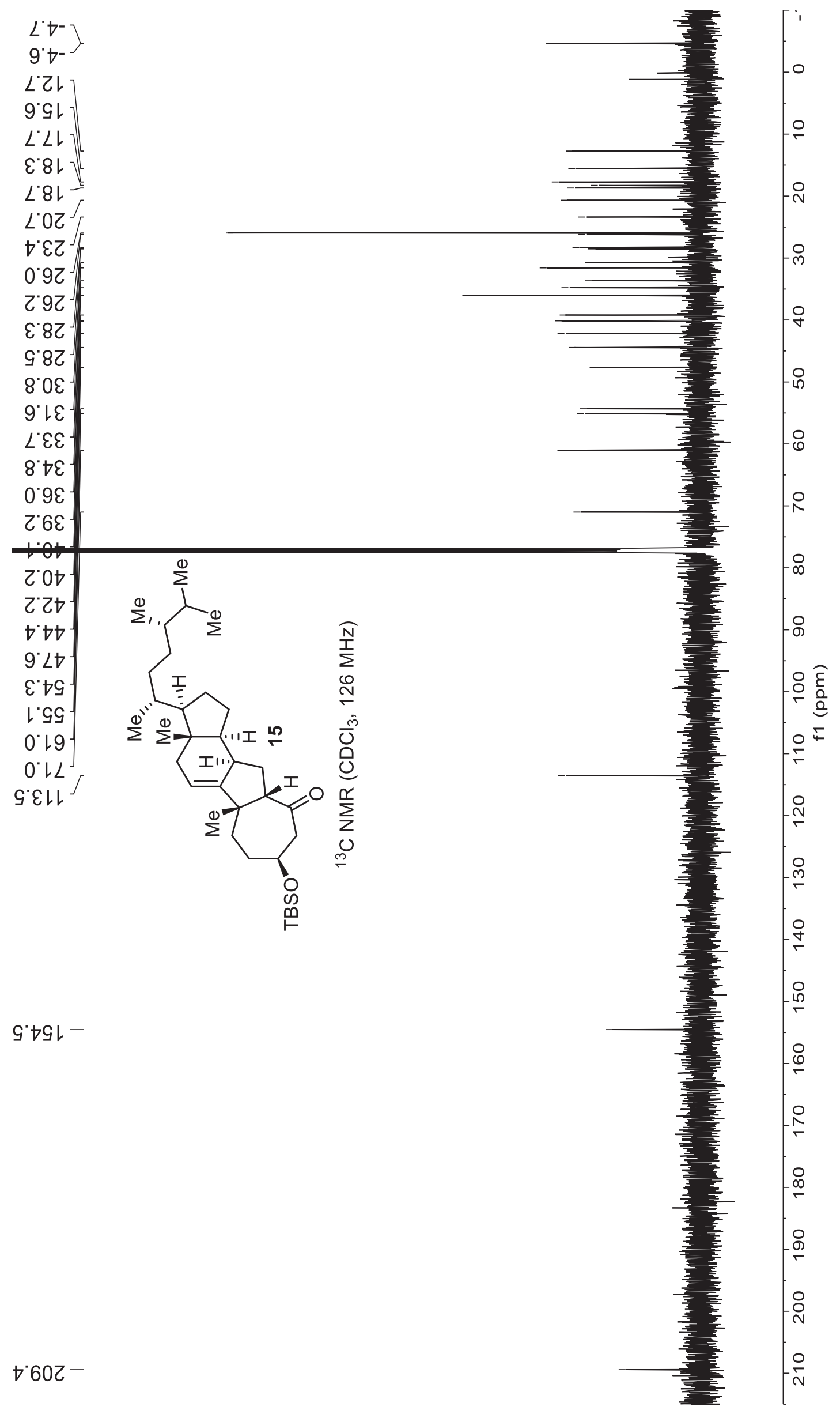




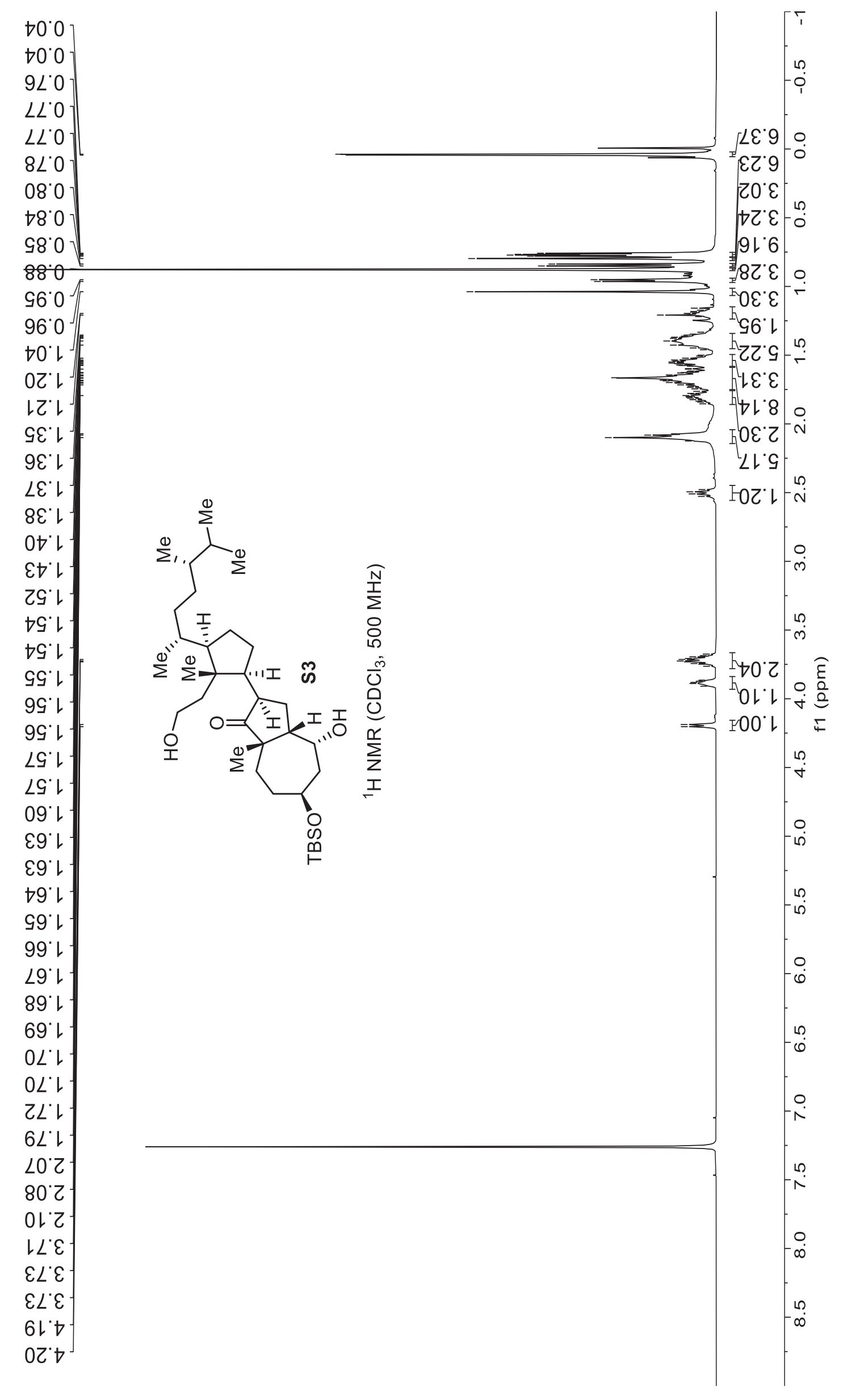




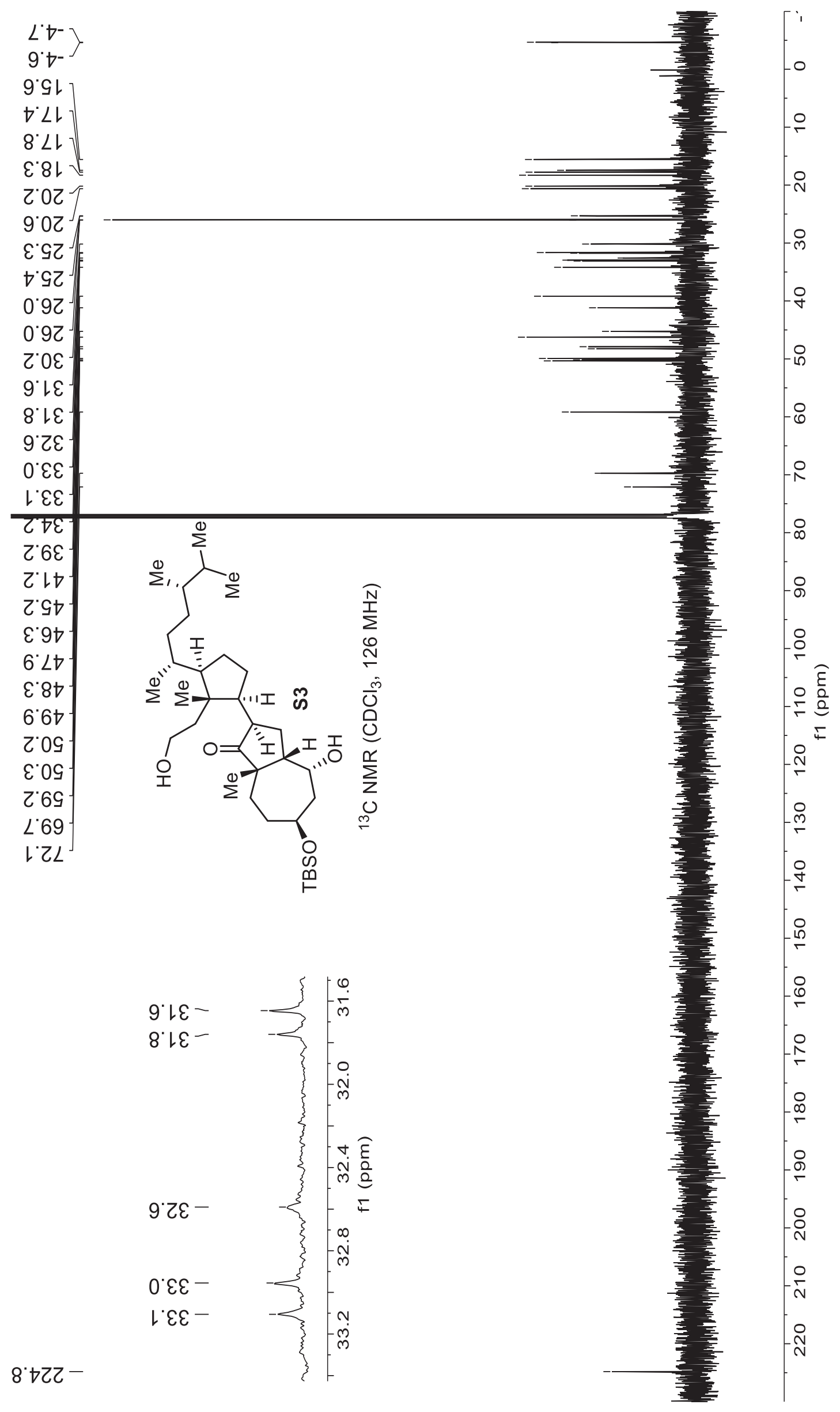




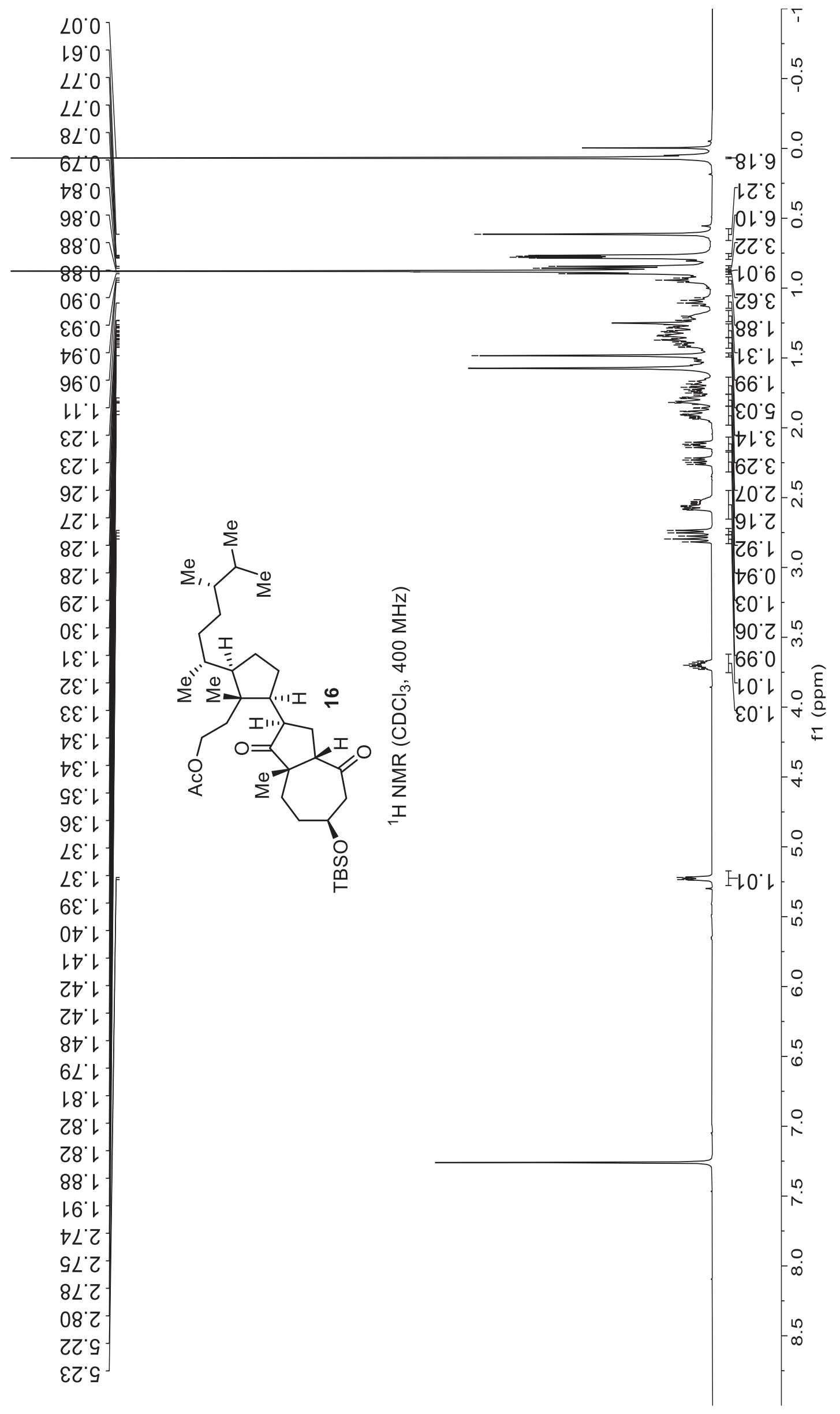




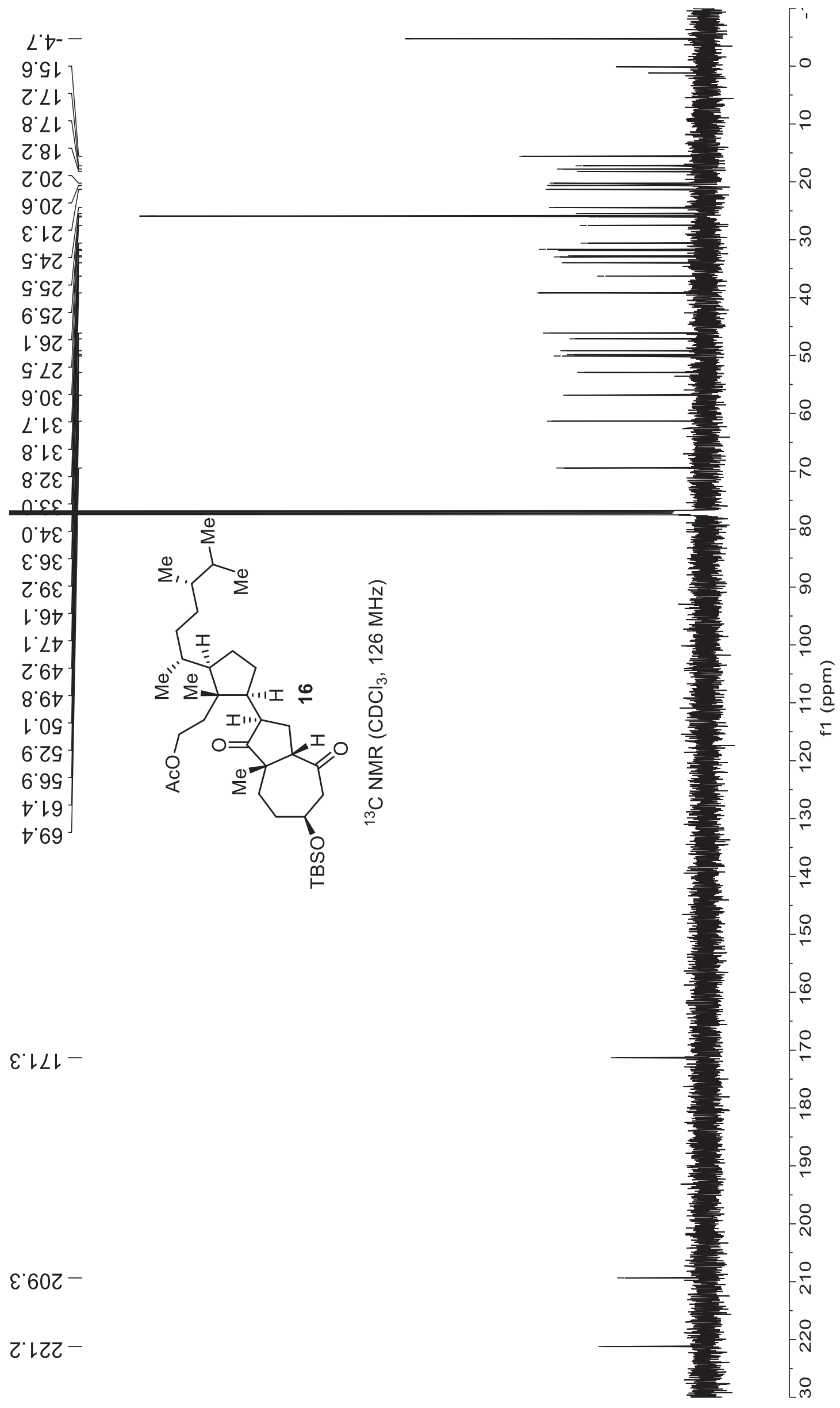




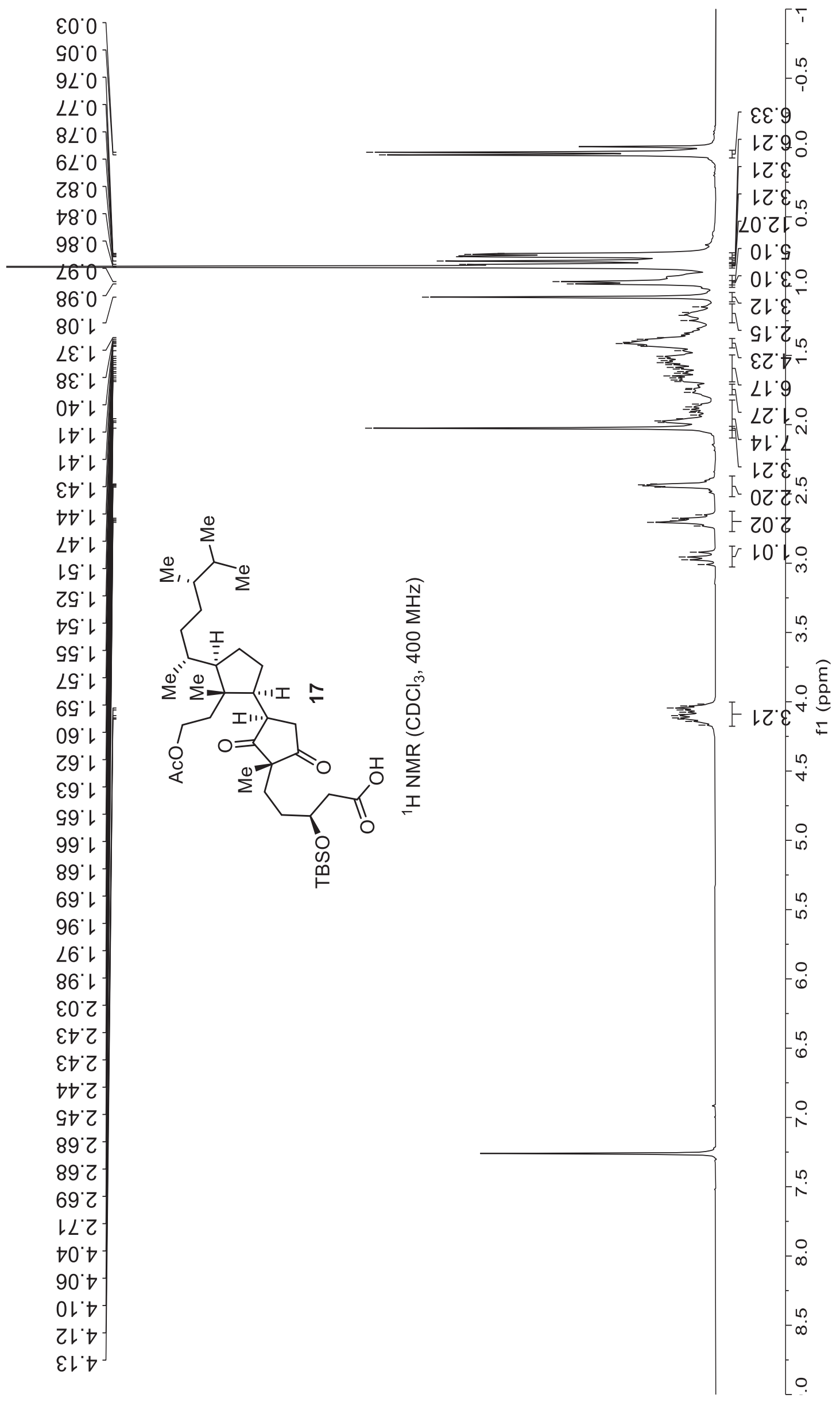




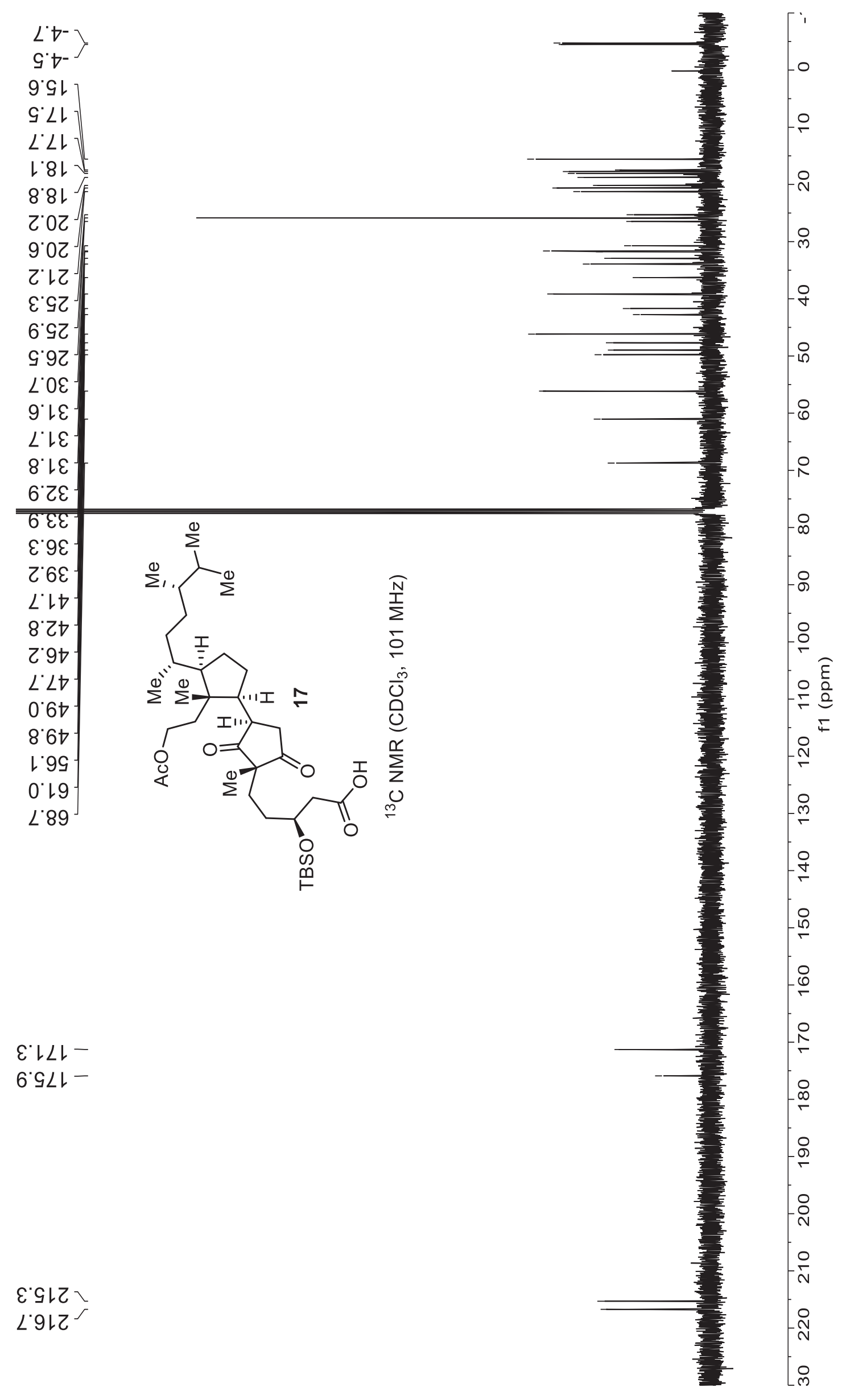




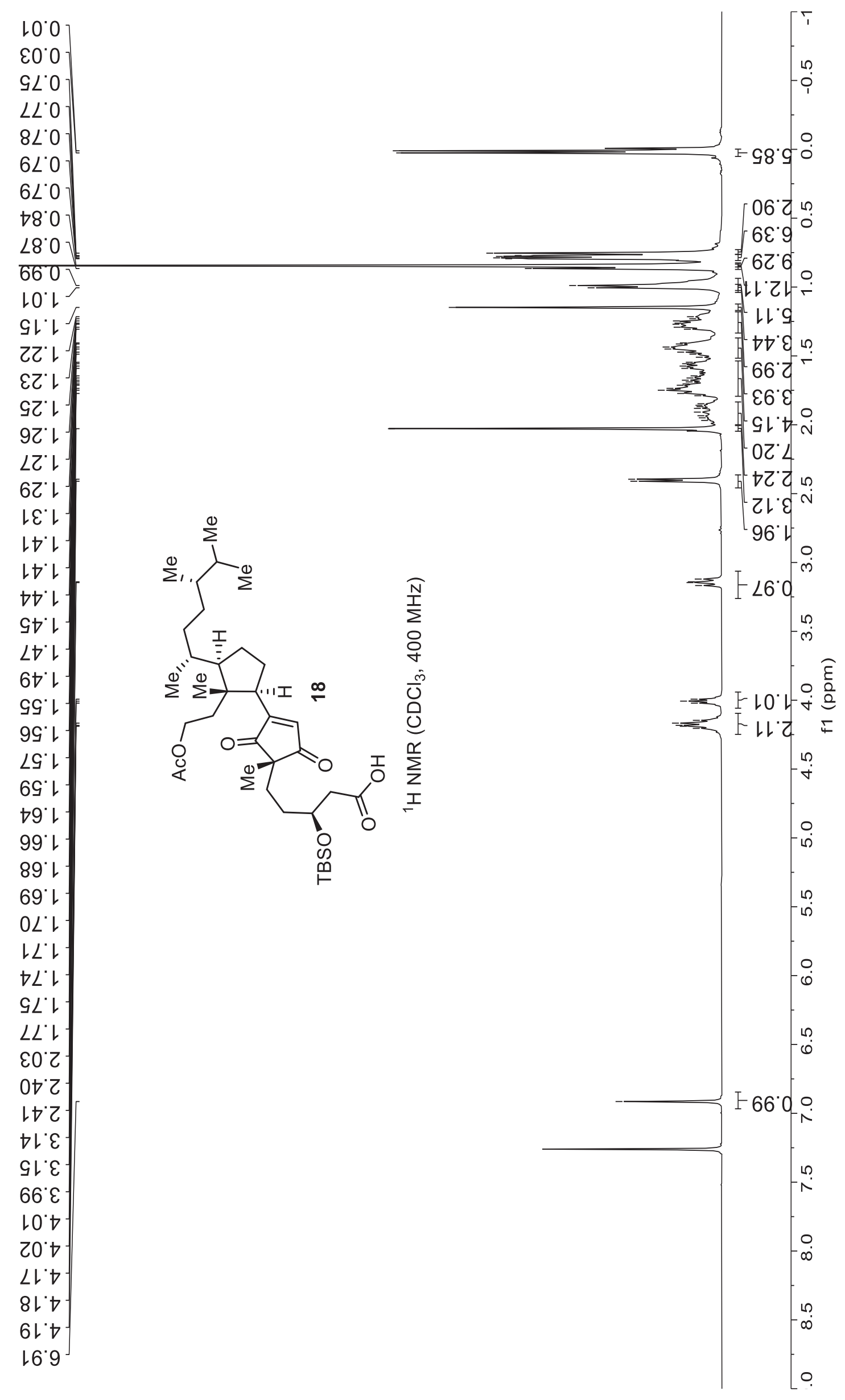




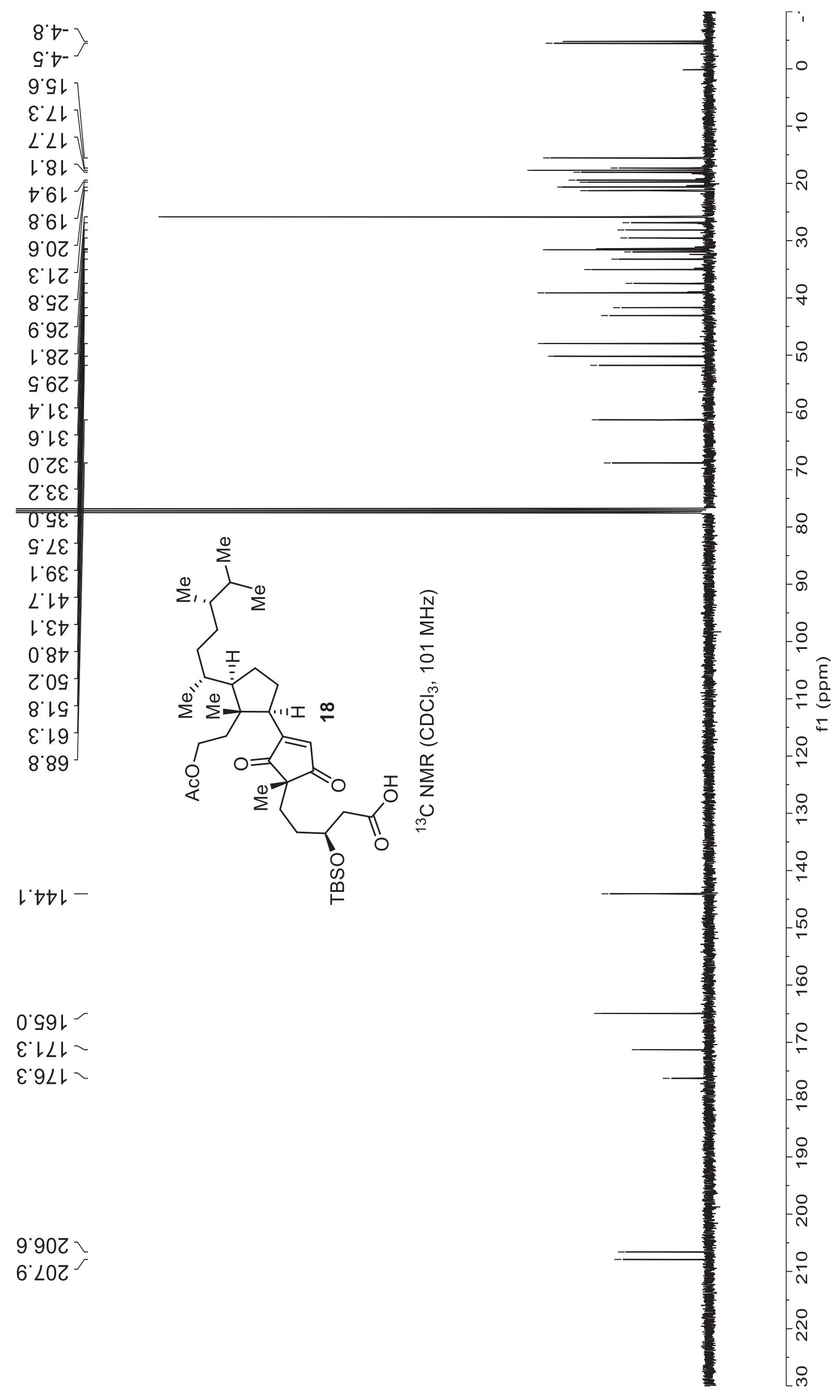



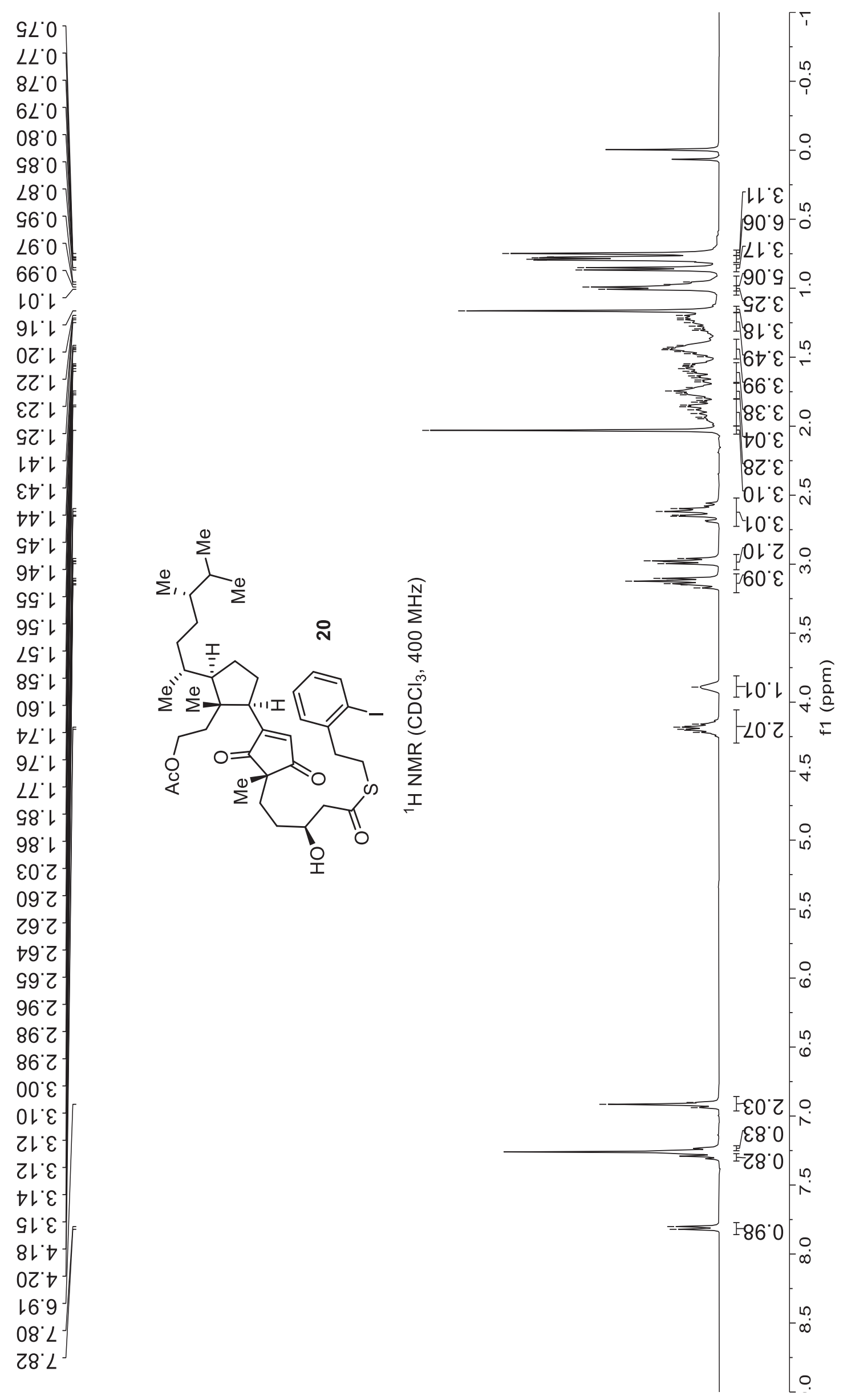


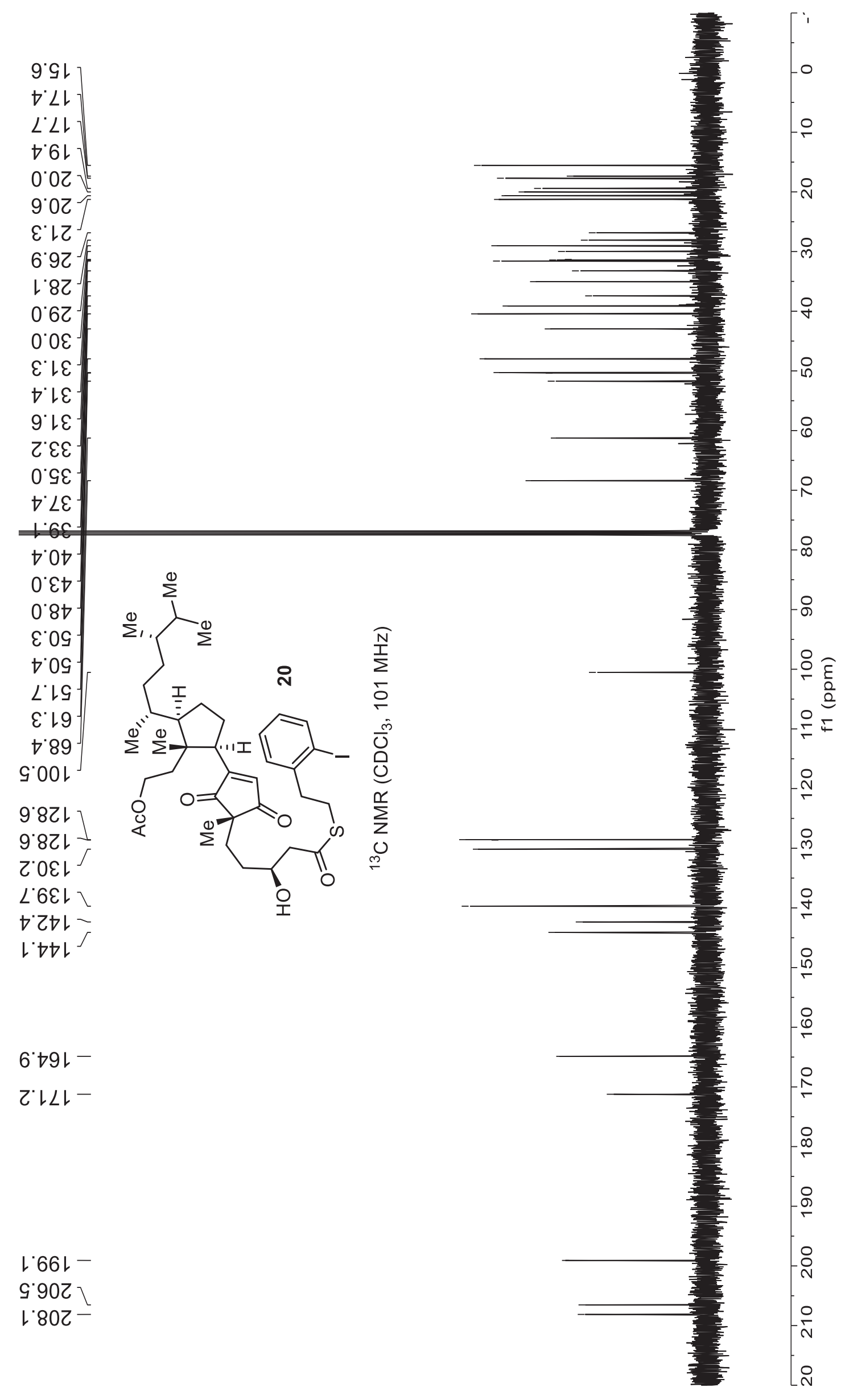




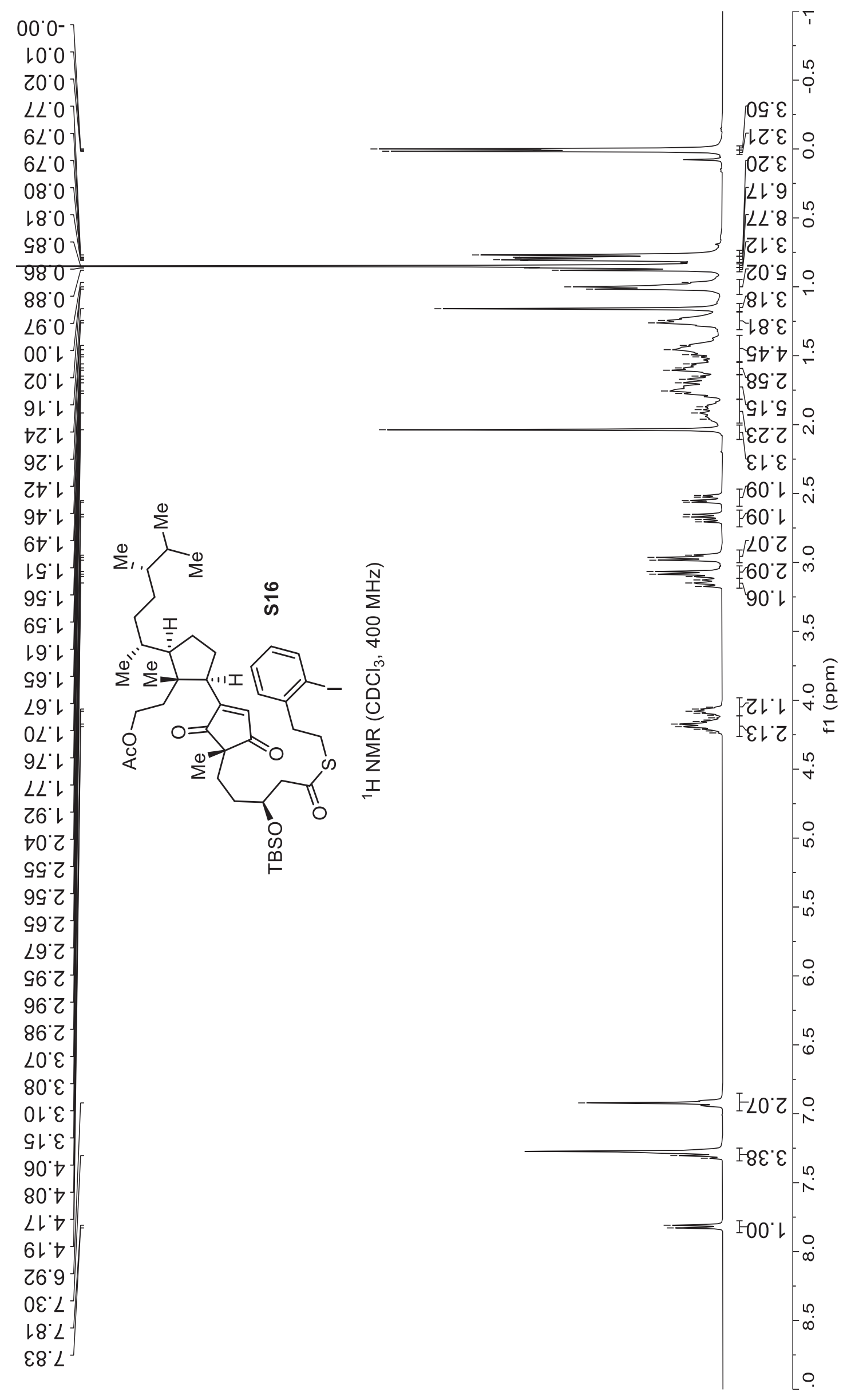




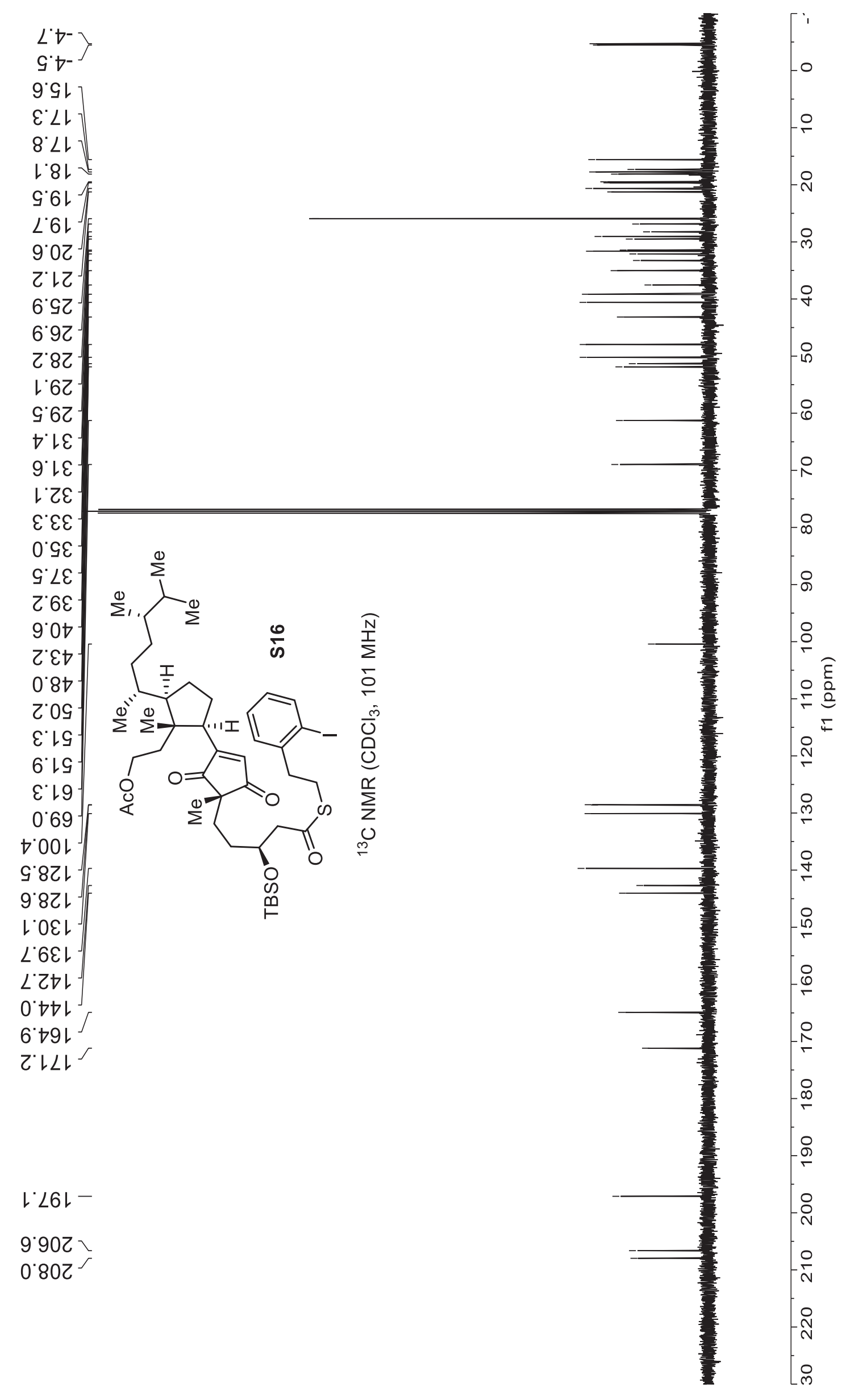




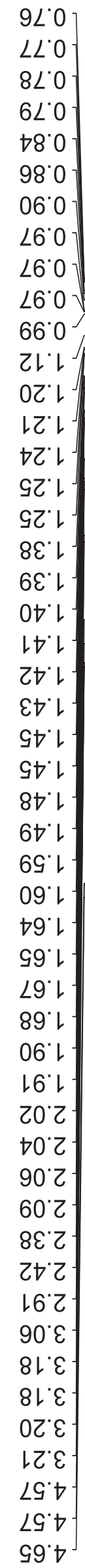

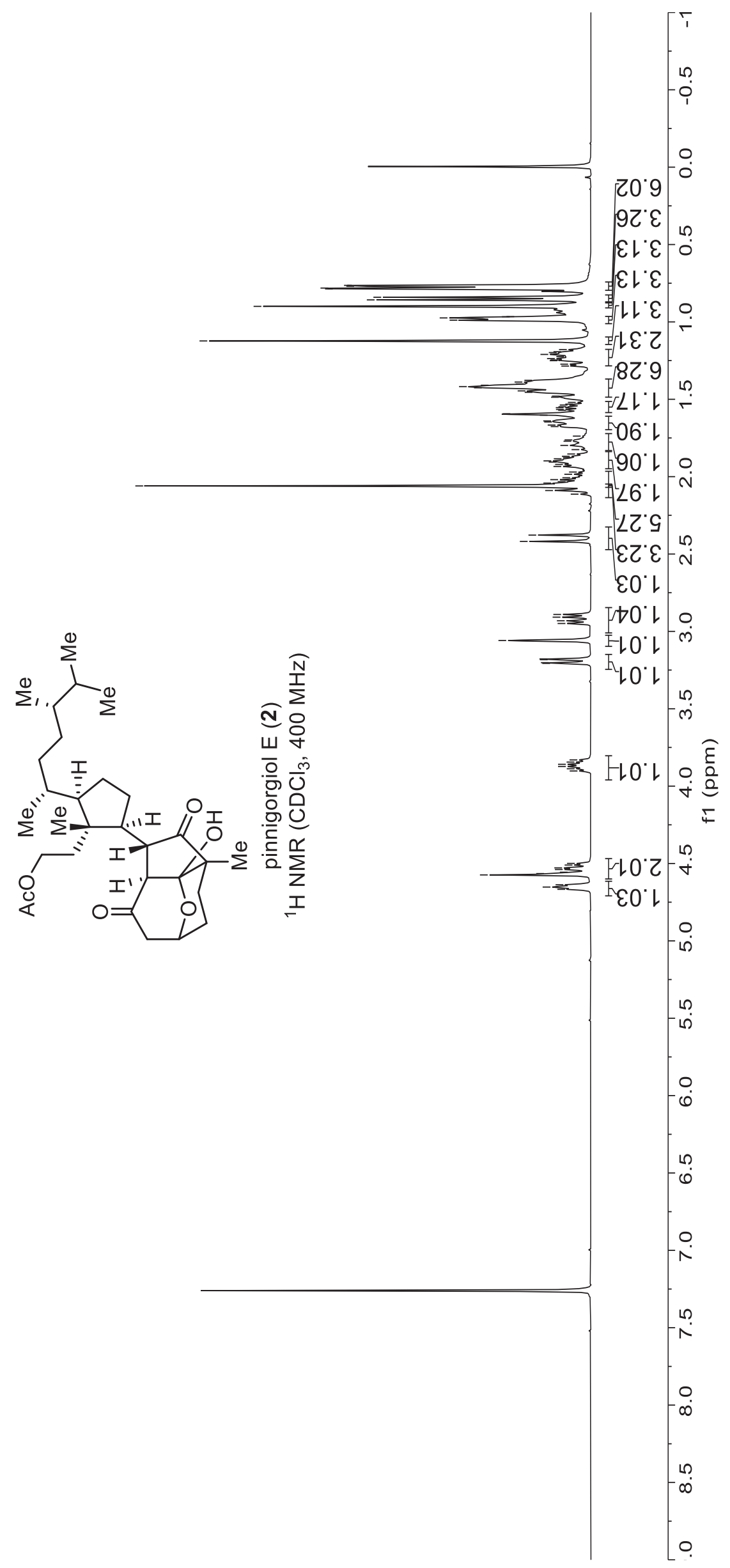




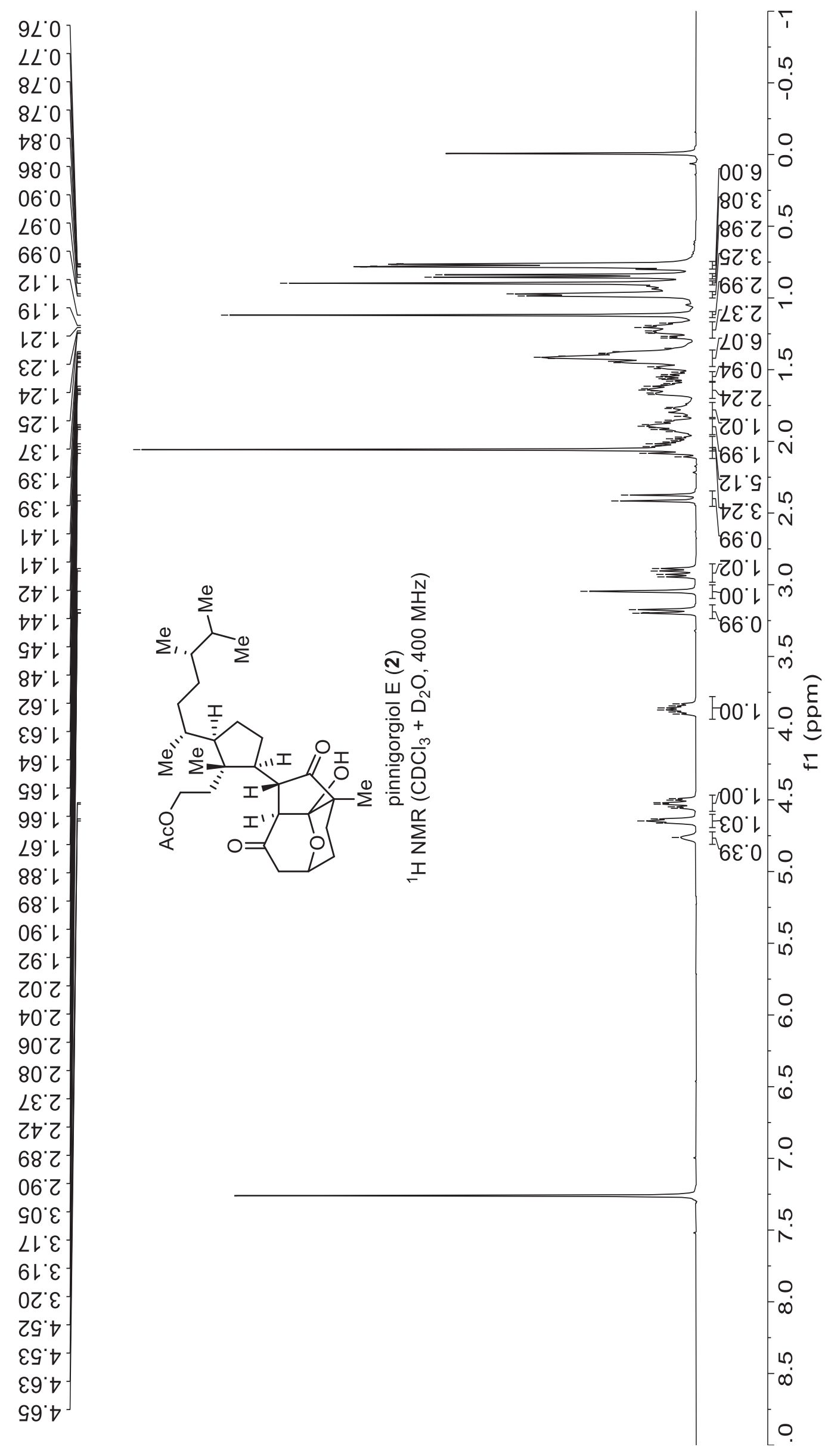




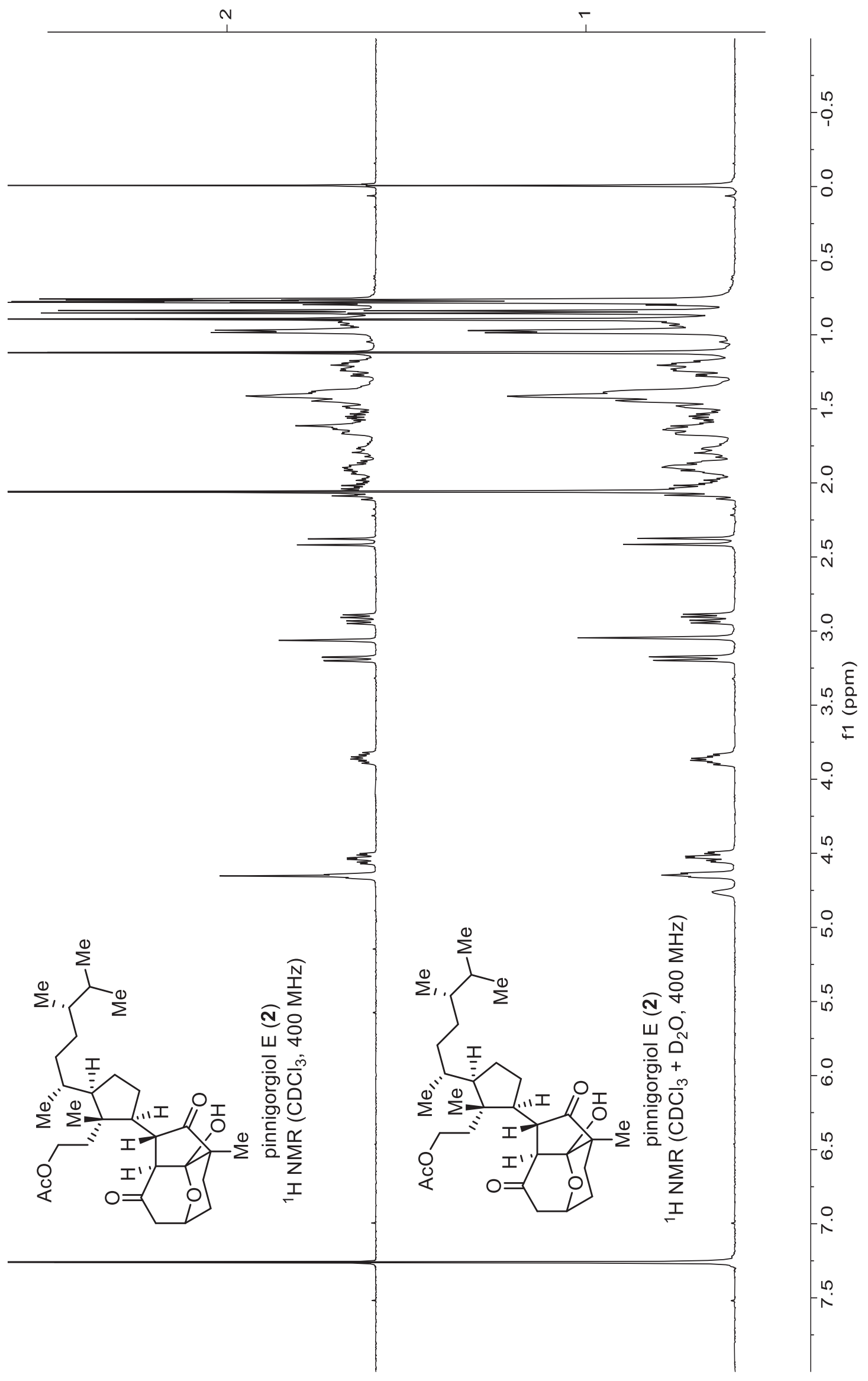




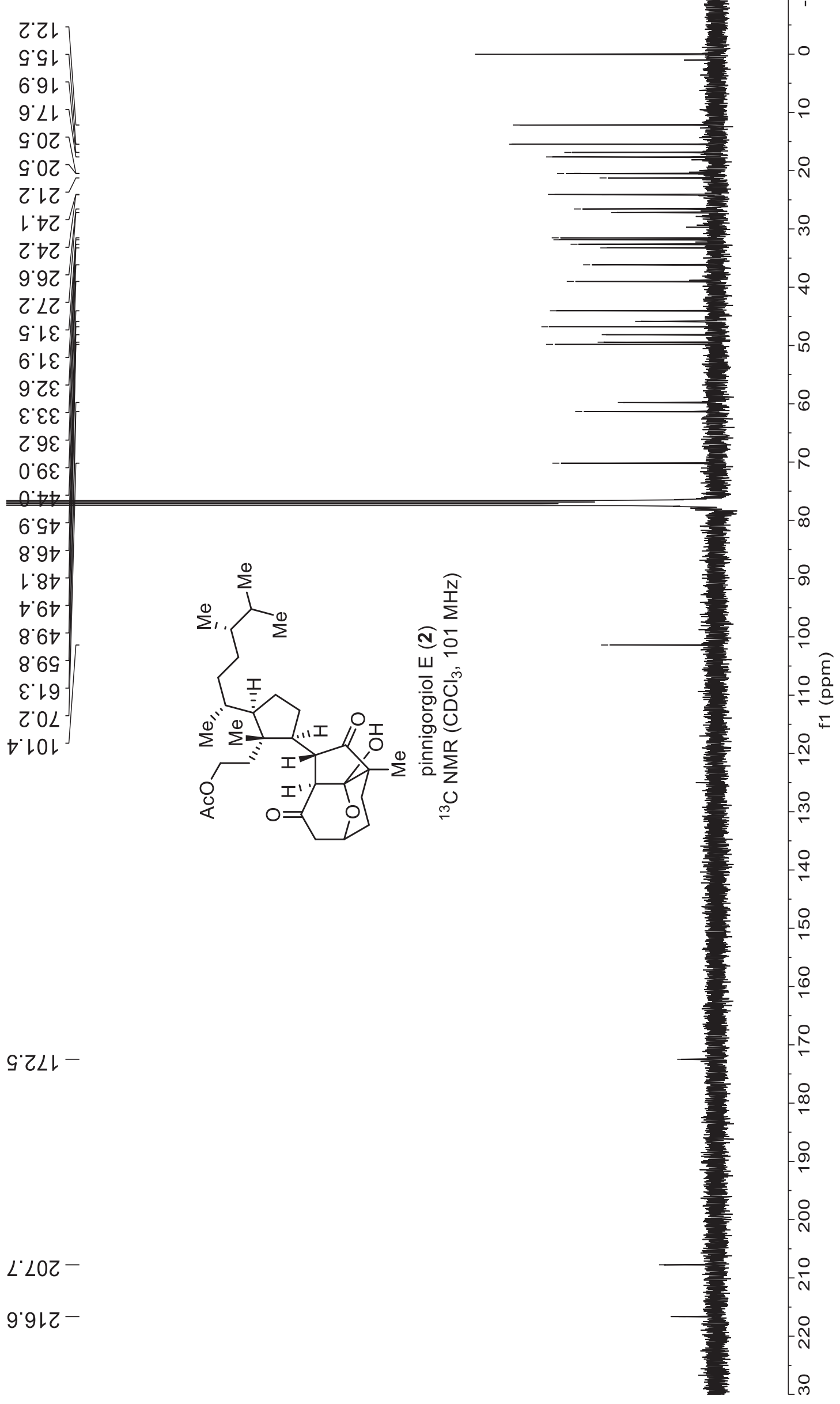




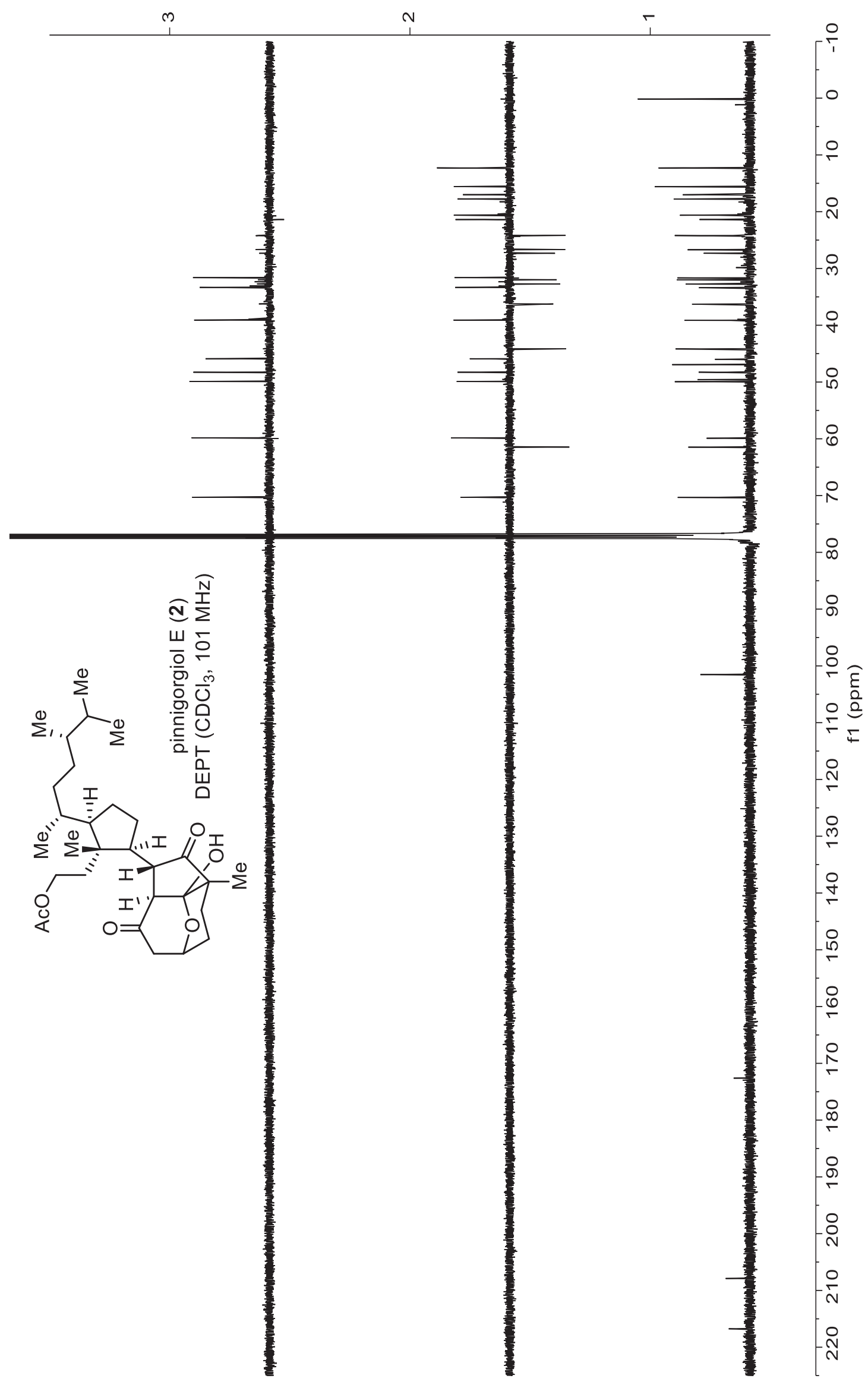

S103 


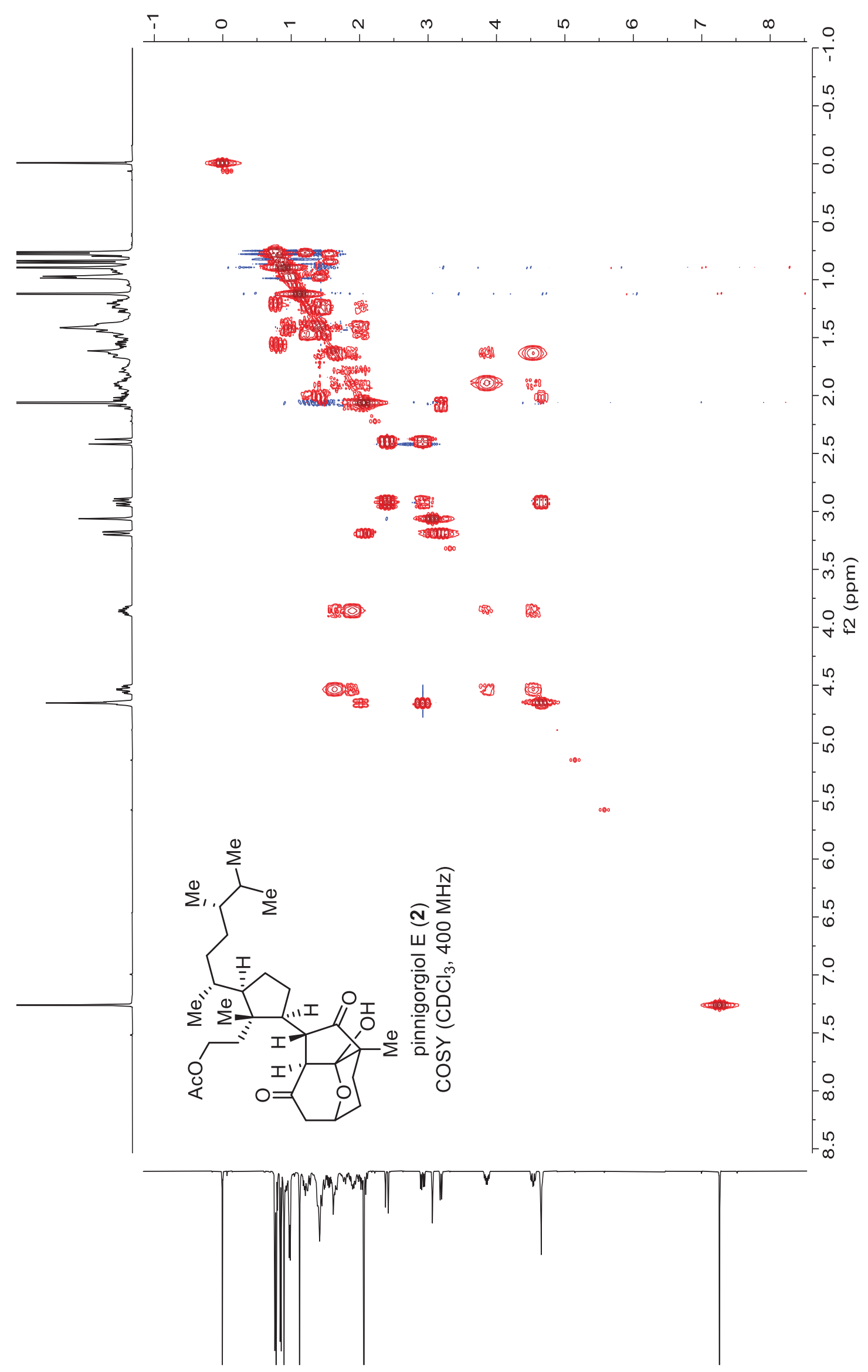




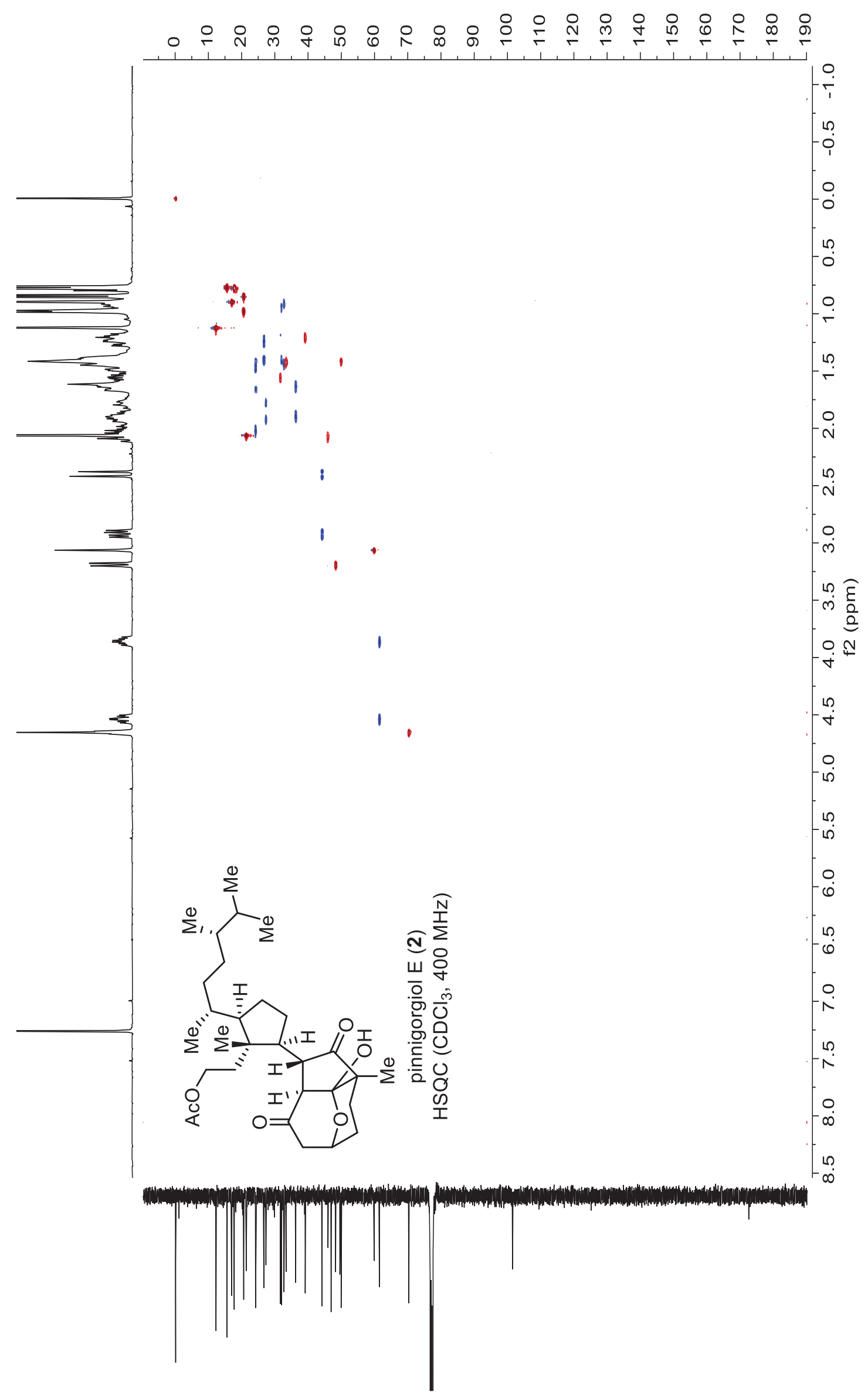




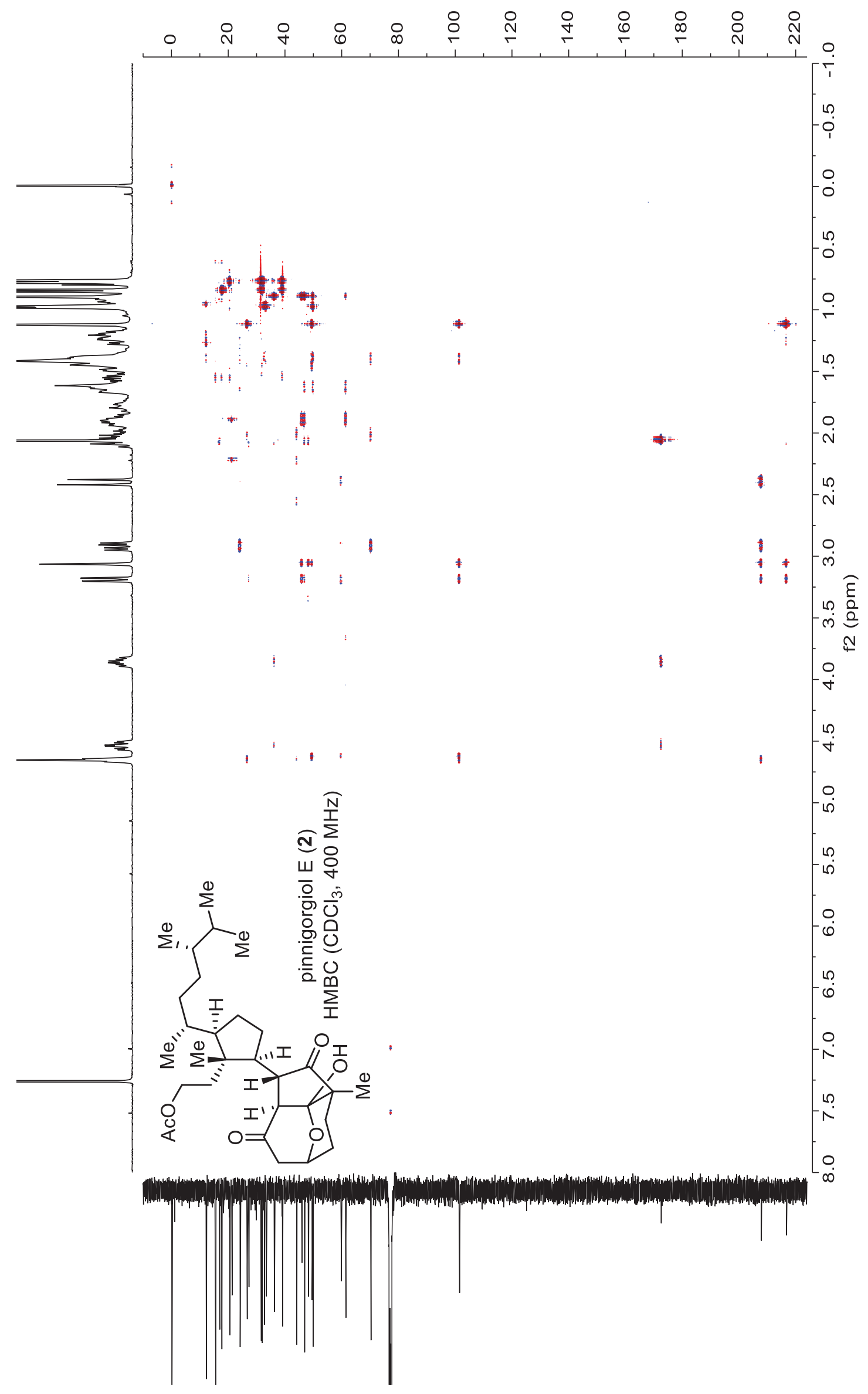




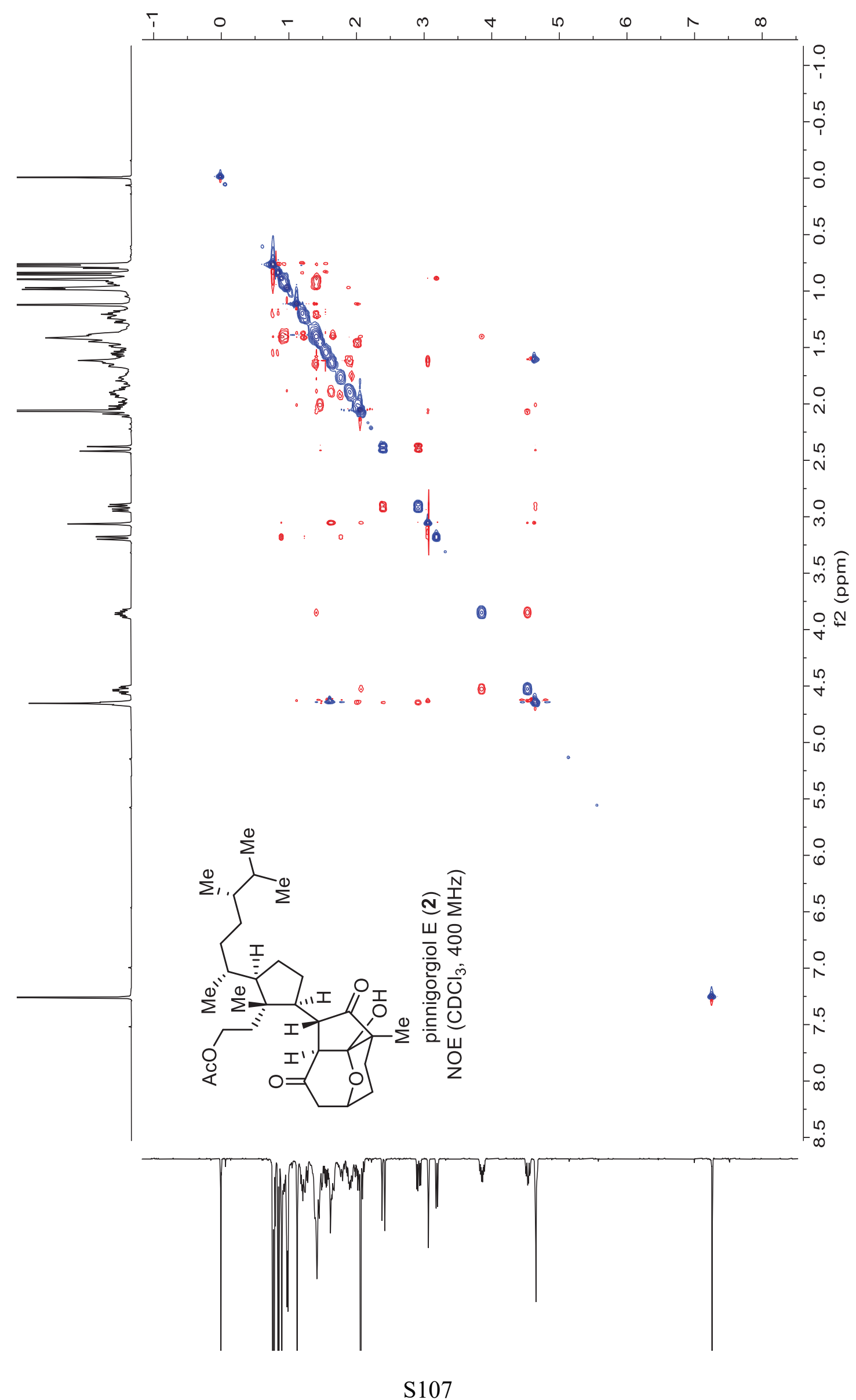




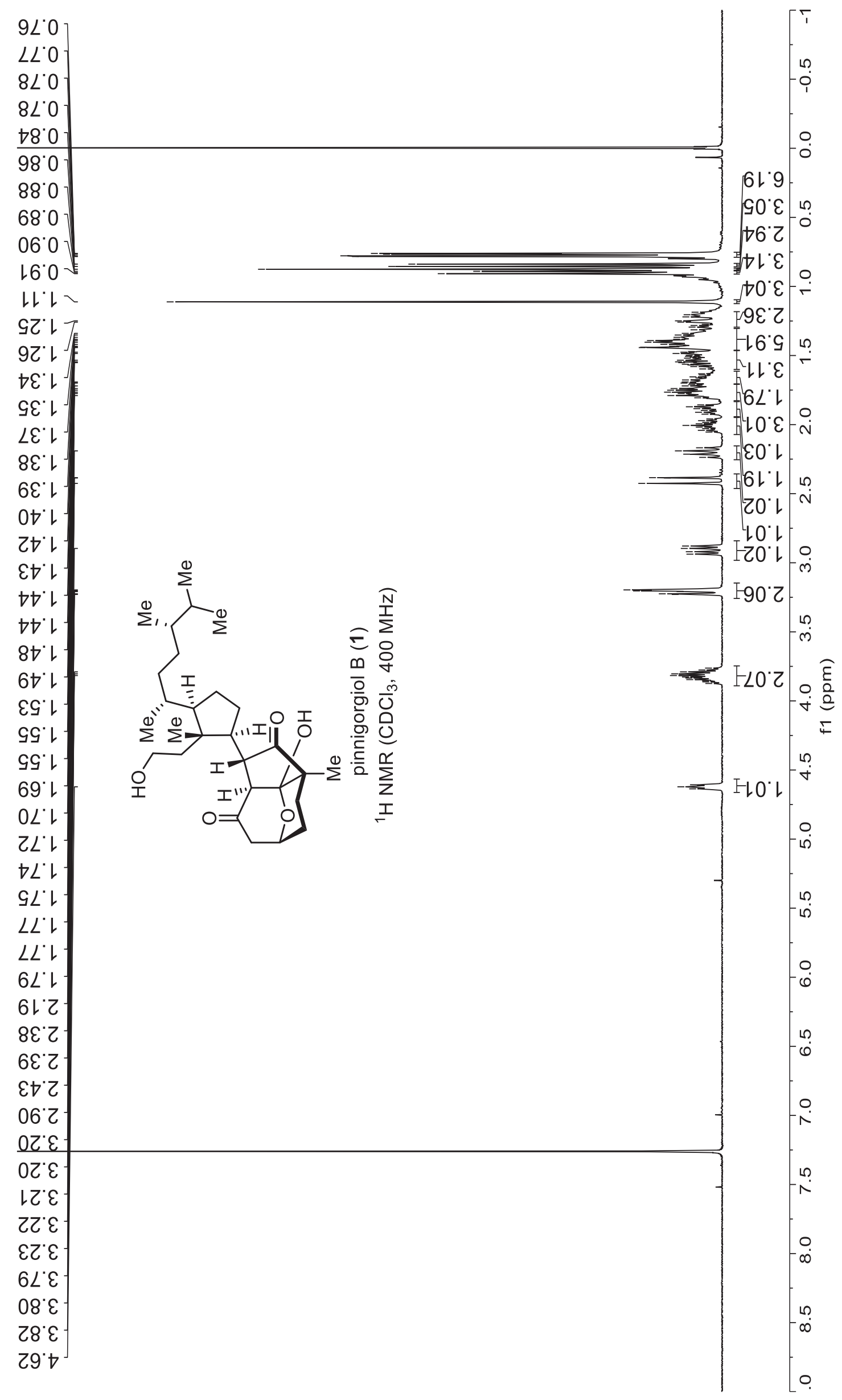




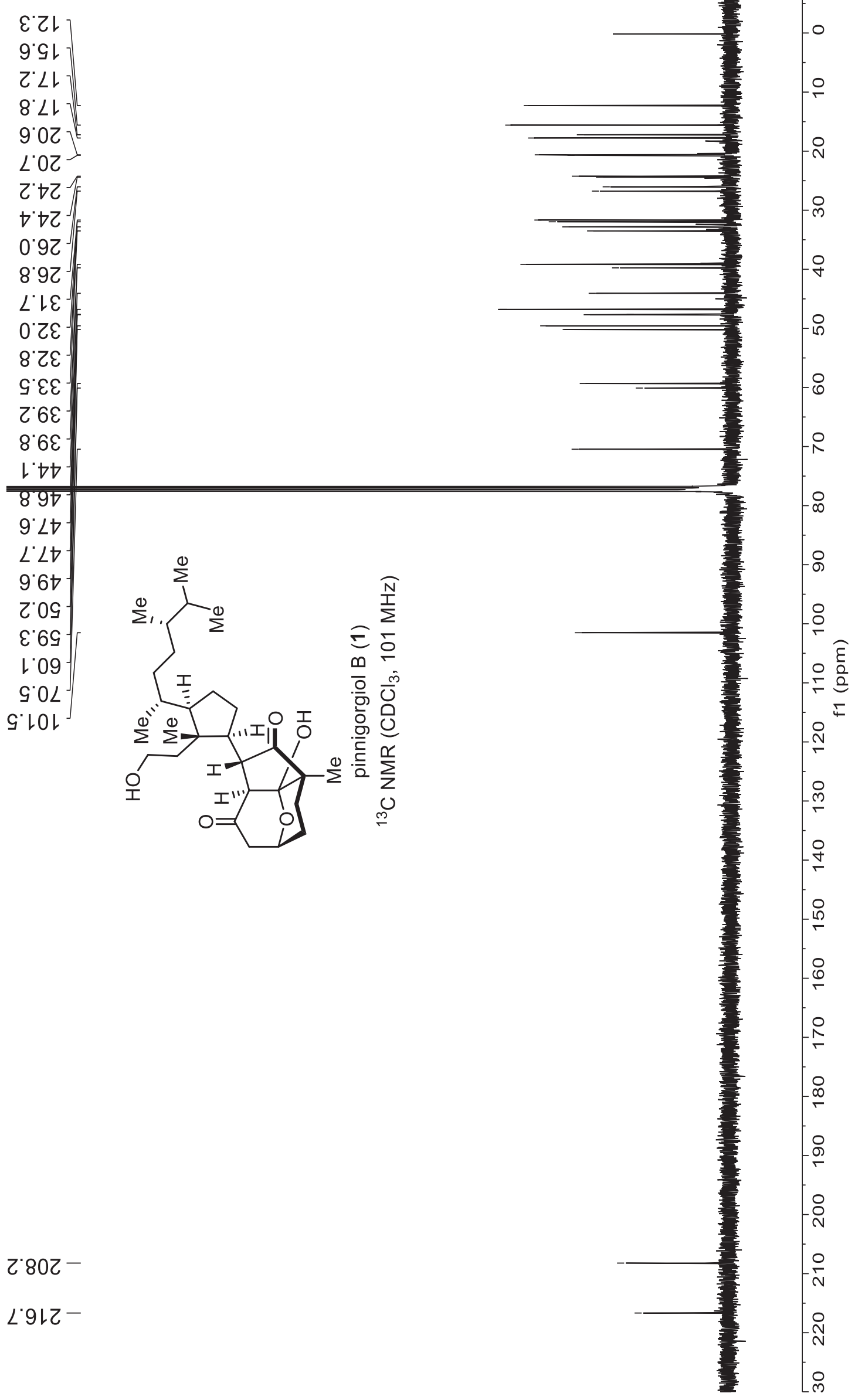




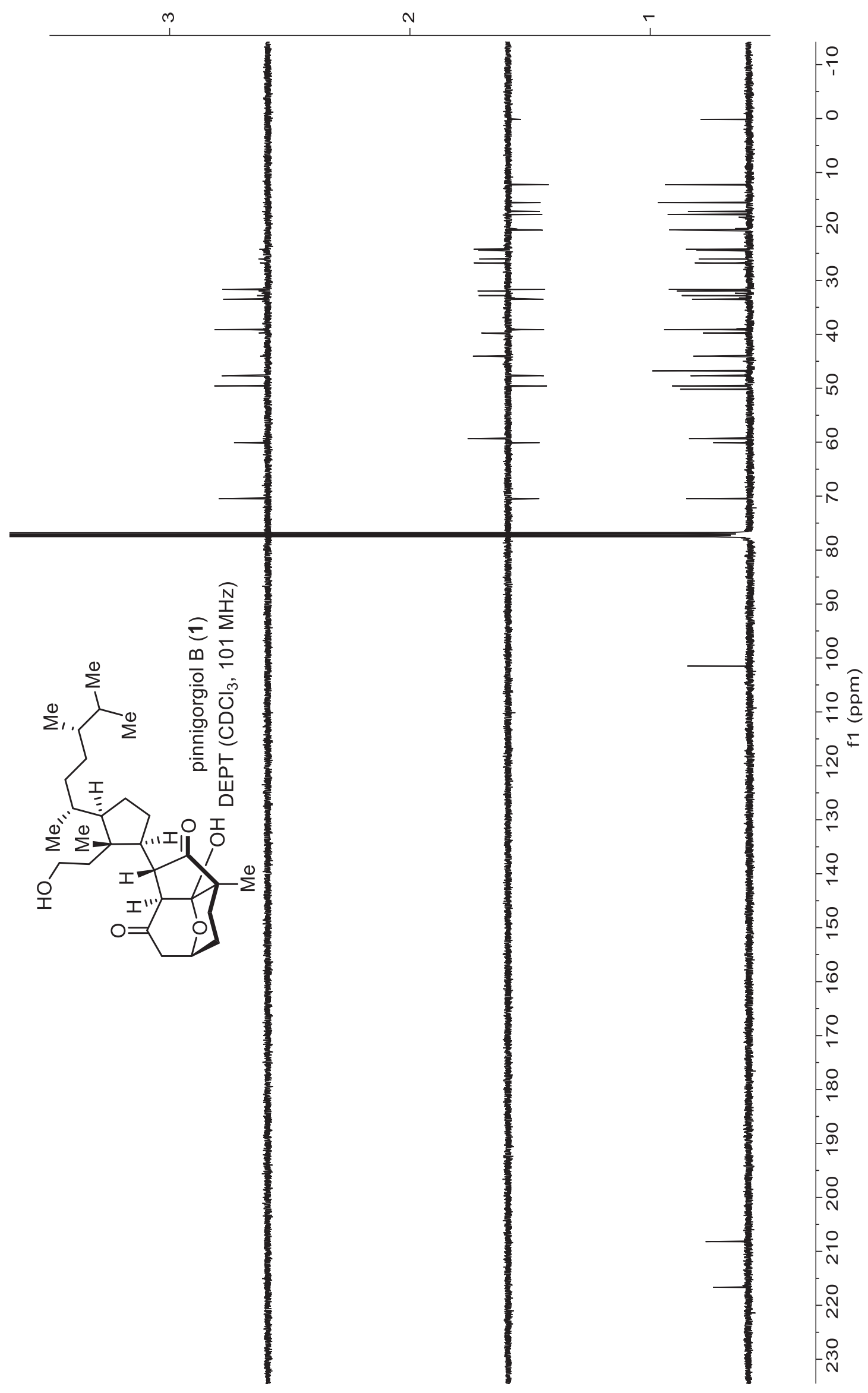

S110 


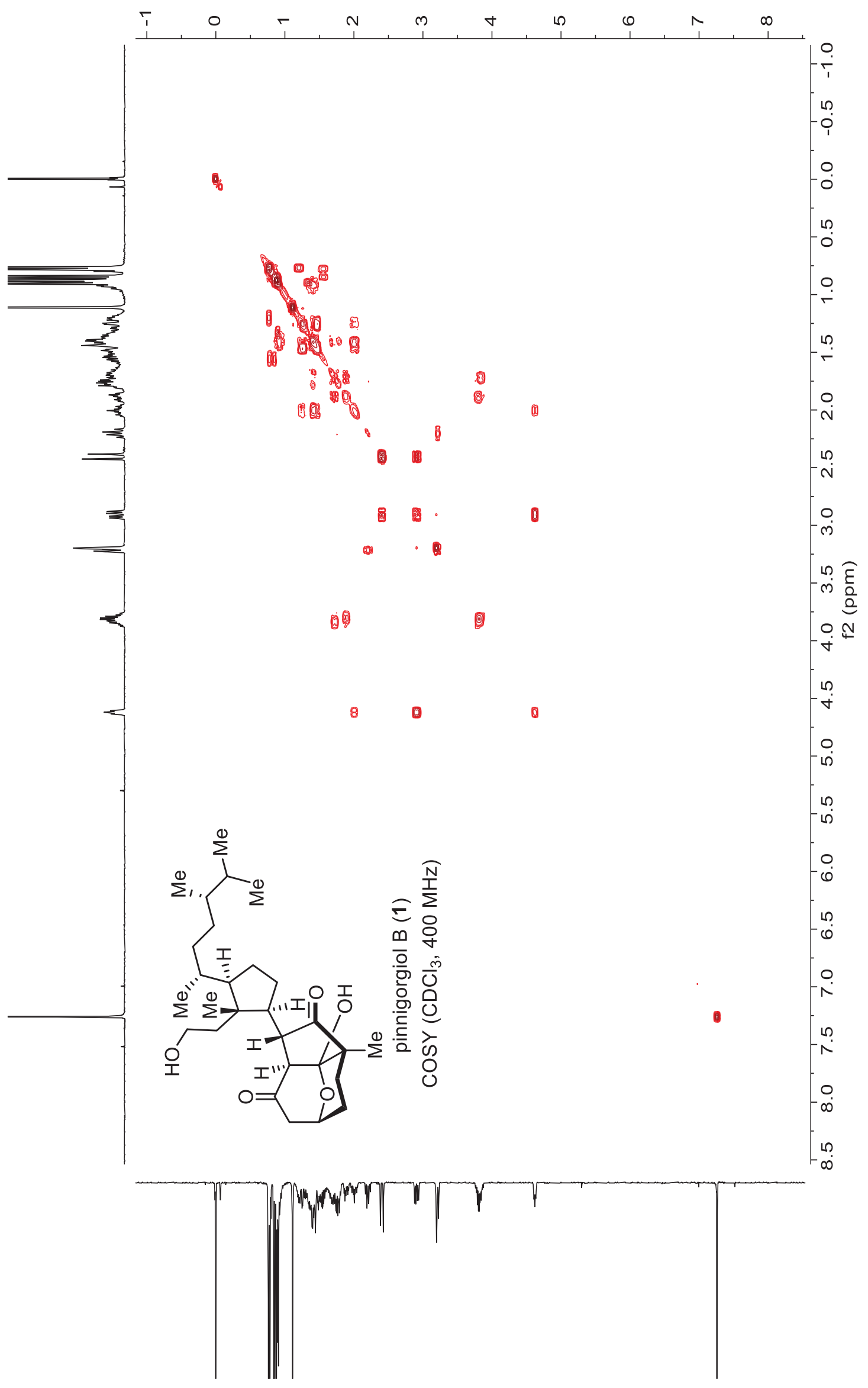


(mdd) Hf

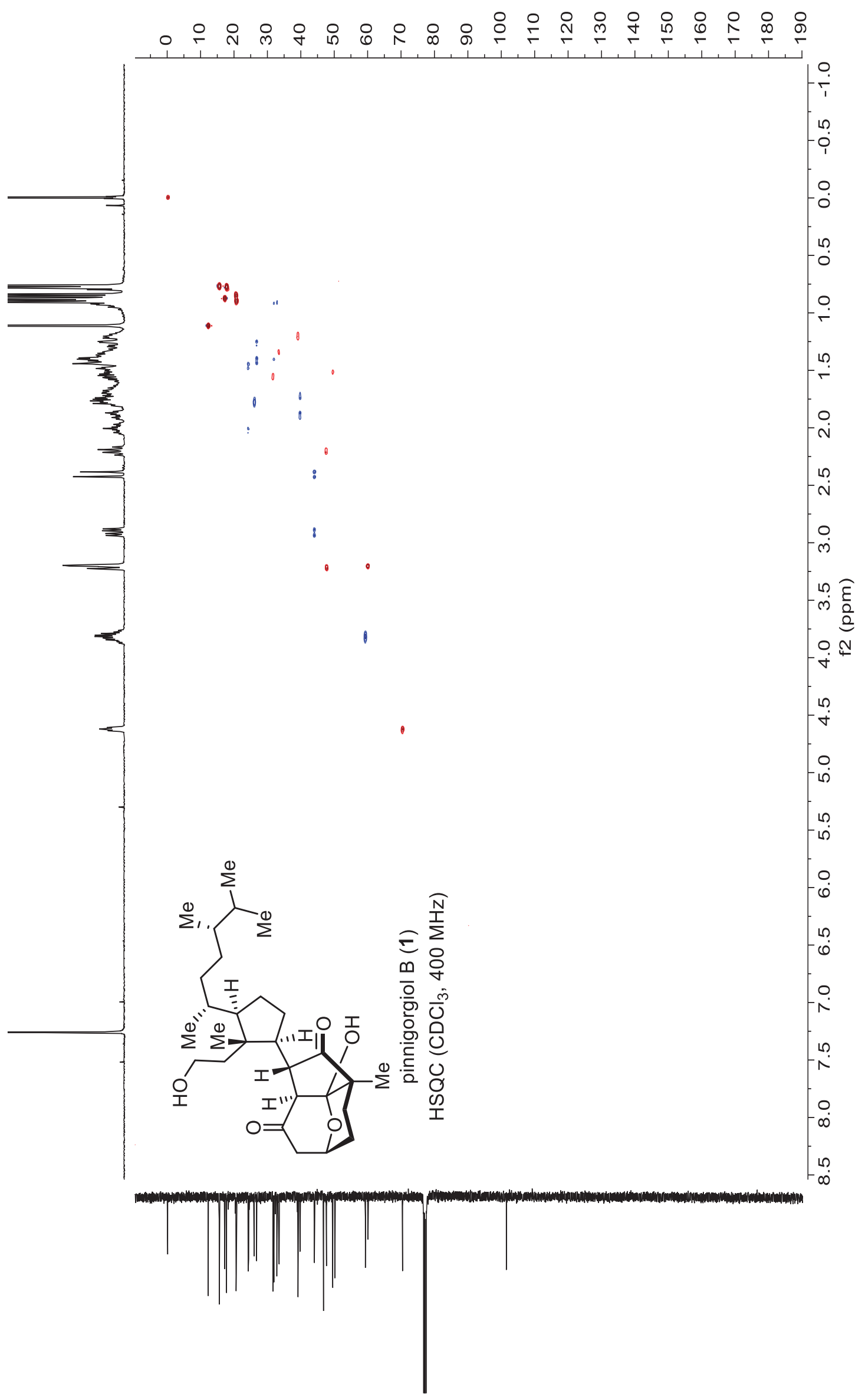


(udd) H

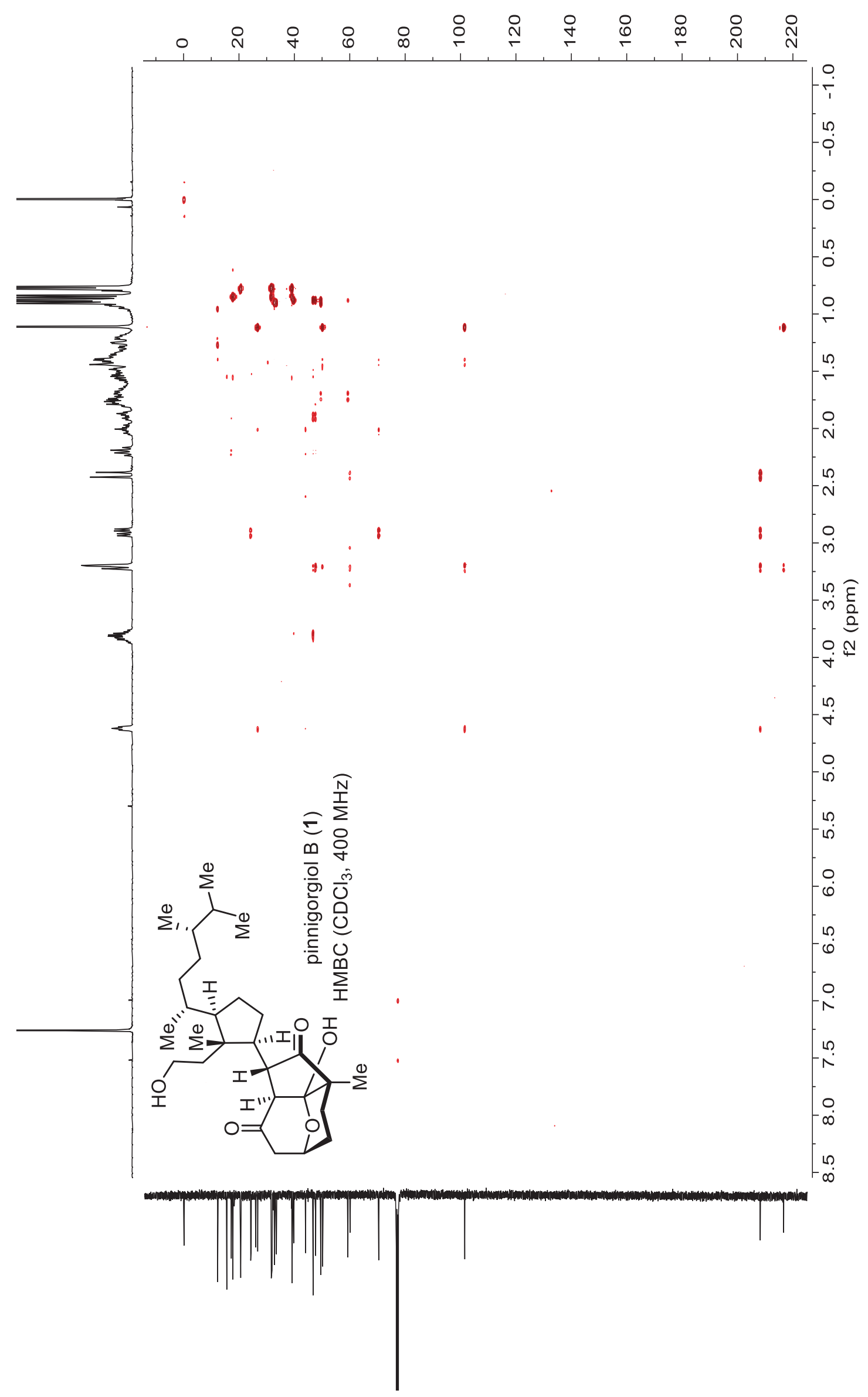




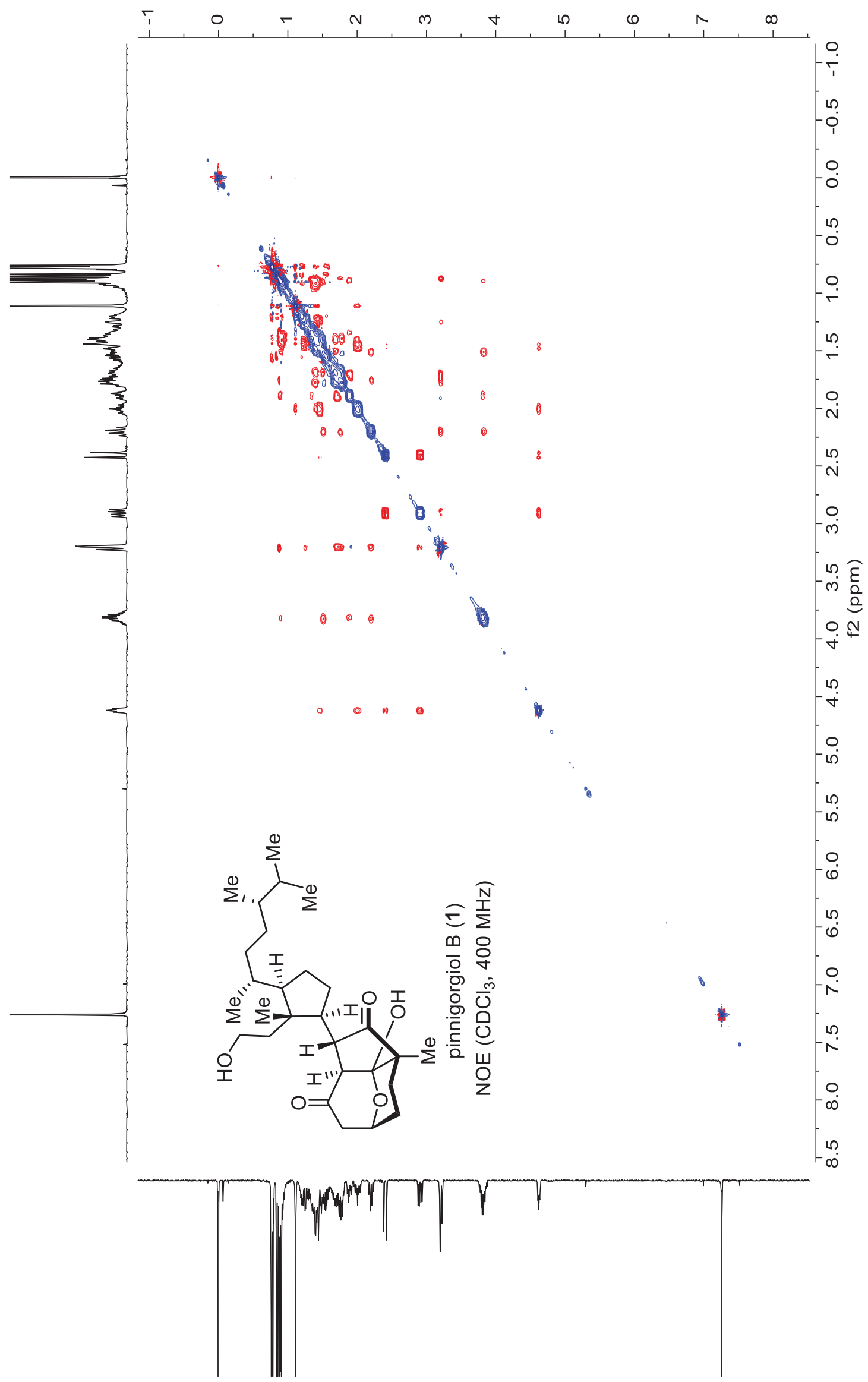

